\title{
INTERACCIÓN SUELO-ESTRUCTURA EN PÉRGOLAS DE FERROCARRIL DE ALTA VELOCIDAD
}

Trabajo Fin de MÁster

\author{
Por \\ Francisco José Cuadros Mantas \\ Máster en Estructuras
}

Tutor

Alejandro E. Martínez Castro
Departamento de Mecánica de Estructuras e Ingeniería Hidráulica , Universidad de Granada
Edificio Politécnico Fuentenueva, C/ Severo Ochoa s/n, CP 18002
Granada (España)

Septiembre 2019 

(C) Copyright 2019

por
Francisco José Cuadros Mantas 



\section{Resumen}

La reciente proliferación de líneas de alta velocidad en España ha provocado la construcción de nuevas líneas ferroviarias prácticamente coincidentes con las ya existentes, lo que ha generado la problemática de cruces entre líneas con un gran esviaje. Aparece la necesidad de diseñar estructuras que salven una luz de gran tamaño, haciendo de las pérgolas la solución óptima al problema.

La finalidad de este trabajo es analizar una pérgola perteneciente a una línea de alta velocidad en servicio y establecer un sistema de modelado en el que se incluya la tipología de cimentación de la estructura. Se pretende calcular la tasa de amortiguamiento geométrico generado a partir de dicha cimentación y evaluar su efecto sobre los resultados dinámicos de la estructura bajo el paso de los trenes indicados en la normativa.

La solución al problema de cargas móviles se ha realizado tradicionalmente mediante la utilización de métodos de integración paso a paso. Dada la ineficacia de dichos métodos para el cálculo exigido en la normativa, en este trabajo se empleará el método semianalítico generalizado a la geometría de una pérgola.

Palabras clave: Análisis dinámico, alta velocidad ferroviaria, pérgola, cimentación mediante pilotes 



\begin{abstract}
The number of high-speed railway lines in Spain has increased a lot in the last years and many of this new lines have been built very close to existing ones, which were built in the 19th century. This has caused the intersection of lines almost parallel. To solve the problem is necessary to build bridges with huge spans and pergola bridges are the optimal solution.

The objetive of this work is to analyze a real high-speed railway pergola bridge and make a modelling that will include the structure and its foundation. It's expected to calculate the geometrical damping generated by the foundation and evaluate its effect in the results of the dynamic analysis.
\end{abstract}

Moving loads problems have usually been solved using step by step methods which are really inefficient. In this work, generalized semi-analytic method will be used to solve them.

Keywords: Dynamic analysis, high-speed rail, pergola bridge, piles foundation 



\section{Agradecimientos}

En primer lugar me gustaría agradecer el apoyo de mis padres. A mis abuelos a los que tanto les debo y que tanto se han preocupado siempre por mí, así como al resto de mi familia. A mis amigos, que han hecho que todos estos años de estudio hayan sido preciosos e inolvidables.

Es esencial agradecer el trabajo de Alejandro E. Martínez Castro, tutor de este trabajo, que no hubiera sido posible sin su dedicación, consejo, guía y apoyo. Y por último agradecer también a Alejandro Castillo Linares por proporcionar los planos de la pérgola de Bobadilla II y así poder estudiarla. 
Dedicado a mis padres y abuelos 


\section{Índice general}

Resumen

III

Abstract

Agradecimientos

VII

Capítulo 1. Introducción, objetivos y metodología 1

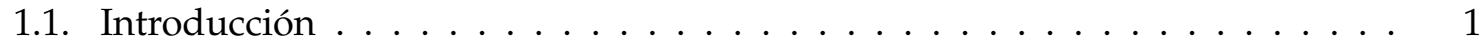

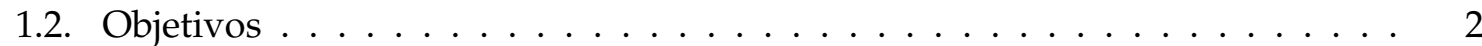

1.3. Metodología .......................... 3

Capítulo 2. Método semianalítico 5

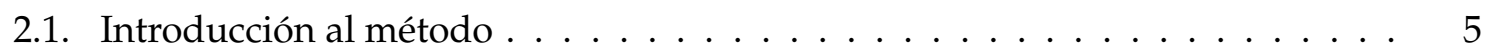

2.2. Ecuación de Gobierno para una viga. . . . . . . . . . . . . . . . 5

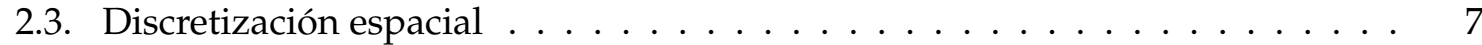

2.4. Resolución . . . . . . . . . . . . . . . . . . . . . . . . 12

2.5. Generalización del método. . . . . . . . . . . . . . . . . . . . . . . 15

2.6. Pruebas de validación $\ldots \ldots \ldots \ldots \ldots \ldots \ldots$

2.6.1. Planteamiento . . . . . . . . . . . . . . . . . 18

2.6.2. Prueba de validación 1: Viga de Bernouilli-Euler biapoyada . . . . . . 18

2.6.3. Prueba de validación 1: Viga de Bernouilli-Euler de tres vanos . . . . . 26

2.7. Conclusiones . . . . . . . . . . . . . . . . . . . . . . . 33

Capítulo 3. Interacción suelo-estructura. Análisis modal 35

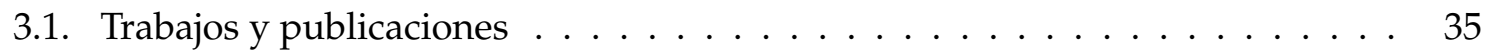

3.2. Características dinámicas de las cimentaciones mediante pilotes . . . . . . . 44

3.2.1. Introducción . . . . . . . . . . . . . . . . . . . 44

3.2.2. Caracterización de la rigidez y el amortiguamiento dinámicos . . . . . 45 
3.2.3. Gráficas de rigidez y amortiguamiento . . . . . . . . . . . . . . 50

3.2.4. Conclusiones . . . . . . . . . . . . . . . . . . 52

3.3. Análisis modal en pérgolas cimentadas por pilotes . . . . . . . . . . . . 53

Capítulo 4. Pérgola de Bobadilla II 55

4.1. Geometría de la pérgola de estudio . . . . . . . . . . . . . . . 55

4.2. Modelo tridimensional de la pérgola . . . . . . . . . . . . . . . . . 57

4.2.1. Cargas muertas . . . . . . . . . . . . . . . 58

4.2.2. Conexión vigas - losa . . . . . . . . . . . . . . . . . . . . . 59

4.2.3. Apoyos . . . . . . . . . . . . . . . . . 60

4.3. Análisis dinámico de la pérgola de Bobadilla II . . . . . . . . . . . . . 61

4.3.1. Datos de partida . . . . . . . . . . . . . . . . 63

4.3.2. Cálculo dinámico . . . . . . . . . . . . . . . . . . 68

4.3.3. Resultados de la interacción suelo - estructura . . . . . . . . . . . . . . 110

$\begin{array}{lll}\text { Capítulo 5. Conclusiones y aportaciones } & 113\end{array}$

Apéndice A. Análisis dinámico de la pérgola de Bobadilla II 115

A.1. Resultado para estructura con apoyos . . . . . . . . . . . . . . 115

A.1.1. Resultados de aceleración . . . . . . . . . . . . . . . . . . . 115

A.1.2. Resultados de desplazamiento . . . . . . . . . . . . . . . 120

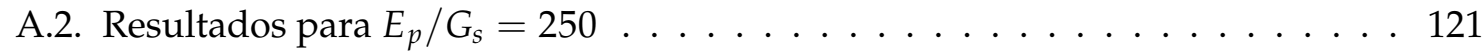

A.2.1. Resultados de aceleración . . . . . . . . . . . . . . . . . . . . . 121

A.2.2. Resultados de desplazamiento . . . . . . . . . . . . . . . 126

A.3. Resultados para $E_{p} / G_{s}=500 \ldots \ldots \ldots \ldots \ldots$. . . . . . . . 127

A.3.1. Resultados de aceleración . . . . . . . . . . . . . . . . . . . 127

A.3.2. Resultados de desplazamiento . . . . . . . . . . . . . . . . . . . 132

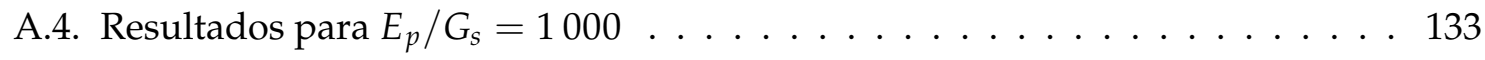

A.4.1. Resultados de aceleración . . . . . . . . . . . . . . . . . 133

A.4.2. Resultados de desplazamiento . . . . . . . . . . . . . . . . . 138

A.5. Resultados para $E_{p} / G_{s}=2500 \ldots \ldots \ldots \ldots \ldots \ldots \ldots$

A.5.1. Resultados de aceleración . . . . . . . . . . . . . . . . . . . . 139

A.5.2. Resultados de desplazamiento . . . . . . . . . . . . . . . . . . 144

A.6. Resultados para $E_{p} / G_{s}=5000 \ldots \ldots \ldots \ldots \ldots \ldots \ldots$ 
A.6.1. Resultados de aceleración . . . . . . . . . . . . . . . . . . . . . 145

A.6.2. Resultados de desplazamiento . . . . . . . . . . . . . . . . . . . . . 150

A.7. Resultados para $E_{p} / G_{s}=10000 \ldots \ldots \ldots \ldots \ldots$. . . . . . . . . 151

A.7.1. Resultados de aceleración . . . . . . . . . . . . . . . . . . . . . . 151

A.7.2. Resultados de desplazamiento . . . . . . . . . . . . . . . . . . . 156 



\section{Índice de cuadros}

2.1. Frecuencias y períodos de vibración. Prueba $1 \ldots \ldots$. . . . . . . . 19

2.2. Frecuencias y períodos de vibración. Prueba $2 \ldots \ldots \ldots$. . . . . 27

4.1. Valores de $f_{v 1}$ para distintos casos de rigidez. Pérgola de Bobadilla II . . . . . 65

4.2. Valores de $f_{v 2}$ para distintos casos de rigidez. Pérgola de Bobadilla II . . . . 66

4.3. Valores de $k_{v}$ y $c_{v}$ para distintos casos de rigidez. Pérgola de Bobadilla II . . . 68

4.4. Valores de período y frecuencia para estructura con apoyos. Pérgola de Bobadilla II . . . . . . . . . . . . . . . . . . . . . . . . . . . . 71

4.5. Máxima aceleración para estrutura con apoyos. Pérgola de Bobadilla II . . . 73

4.6. Máximo desplazamiento para estrutura con apoyos. Pérgola de Bobadilla II . 74

4.7. Valores de período, frecuencia y amortiguamiento para $E_{p} / G_{s}=250$. Pérgola de Bobadilla II . . . . . . . . . . . . . . . . . . . . . . . . 78

4.8. Máxima aceleración para $E_{p} / G_{s}=250$. Pérgola de Bobadilla II $\ldots \ldots \ldots$

4.9. Máximo desplazamiento para $E_{p} / G_{s}=250$. Pérgola de Bobadilla II $\ldots \ldots$. . 79

4.10. Valores de período, frecuencia y amortiguamiento para $E_{p} / G_{s}=500$. Pérgola de Bobadilla II . . . . . . . . . . . . . . . . . . . . . . 84

4.11. Máxima aceleración para $E_{p} / G_{s}=500$. Pérgola de Bobadilla II . . . . . . . 84

4.12. Máximo desplazamiento para $E_{p} / G_{s}=500$. Pérgola de Bobadilla II $\ldots \ldots$. . 85

4.13. Valores de período, frecuencia y amortiguamiento para $E_{p} / G_{s}=1000$. Pérgola de Bobadilla II . . . . . . . . . . . . . . . . . . . . . . 90

4.14. Máxima aceleración para $E_{p} / G_{s}=1000$. Pérgola de Bobadilla II $\ldots \ldots \ldots$

4.15. Máximo desplazamiento para $E_{p} / G_{s}=1000$. Pérgola de Bobadilla II . . . . . 91

4.16. Valores de período, frecuencia y amortiguamiento para $E_{p} / G_{s}=2500$. Pérgola de Bobadilla II . . . . . . . . . . . . . . . . . . . . . . . . . 96

4.17. Máxima aceleración para $E_{p} / G_{s}=2500$. Pérgola de Bobadilla II $\ldots \ldots \ldots$

4.18. Máximo desplazamiento para $E_{p} / G_{s}=2500$. Pérgola de Bobadilla II . . . . 97

4.19. Valores de período, frecuencia y amortiguamiento para $E_{p} / G_{s}=5000$. Pérgola de Bobadilla II . . . . . . . . . . . . . . . . . . . . . . . . . 102 
4.20. Máxima aceleración para $E_{p} / G_{s}=5000$. Pérgola de Bobadilla II $\ldots \ldots \ldots$

4.21. Máximo desplazamiento para $E_{p} / G_{s}=5$ 000. Pérgola de Bobadilla II $\ldots \ldots$

4.22. Valores de período, frecuencia y amortiguamiento para $E_{p} / G_{s}=10000$. Pérgo-

la de Bobadilla II . . . . . . . . . . . . . . . . . . . . . . . . . . . 108

4.23. Máxima aceleración para $E_{p} / G_{s}=10000$. Pérgola de Bobadilla II . . . . . . . 108

4.24. Máximo desplazamiento para $E_{p} / G_{s}=10$ 000. Pérgola de Bobadilla II . . . . . 109 


\section{Índice de figuras}

1.1. Esquema de una pérgola ferroviaria. Ugarte González [37] . . . . . . . . . . 2

2.1. Esquema de carga móvil sobre viga de sección variable. García Macías [5] . 6

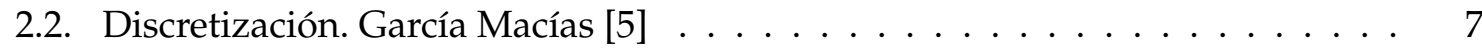

2.3. Esquema de línea de carga C1. García Macías [5] . . . . . . . . . . . . . . . . 16

2.4. Esquema de línea de carga y puntos de postproceso en estructura. García Macías

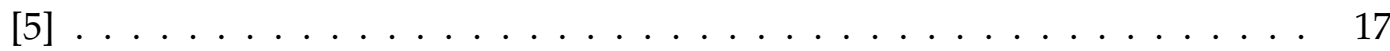

2.5. Esquema de cálculo del Método Semianalítico Generalizado. García Macías [5] 17

2.6. Geometría de la viga. Prueba 1. . . . . . . . . . . . . . . . . . . . . . 18

2.7. Desplazamientos en centro de vano de manera analítica. Prueba 1 . . . . . . 20

2.8. Aceleración de centro de vano de manera analítica. Prueba $1 \ldots$. . . . . . . 21

2.9. Desplazamientos en centro de vano mediante SAP2000. Prueba $1 \ldots$. . . . . 22

2.10. Aceleración en centro de vano mediante SAP2000. Prueba $1 \ldots \ldots$

2.11. Desplazamientos en centro de vano mediante el método semianalítico. Prue-

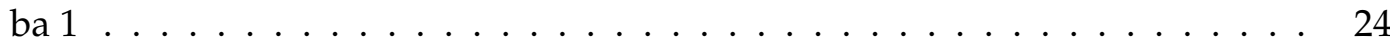

2.12. Aceleración en centro de vano mediante el método semianalítico. Prueba 1 . . 25

2.13. Comparativa de desplazamientos. Prueba $1 \ldots \ldots$. . . . . . . . . . . 25

2.14. Comparativa de aceleración. Prueba $1 \ldots \ldots \ldots$

2.15. Geometría de la viga. Prueba $2 \ldots \ldots \ldots$

2.16. Desplazamientos en centro de vano central de SAP2000. Prueba $2 \ldots \ldots$

2.17. Aceleración en centro de vano central de SAP2000. Prueba $2 \ldots \ldots$

2.18. Desplazamientos en centro de vano central del método semianalítico. Prueba 230

2.19. Aceleración en centro de vano central del método semianalítico. Prueba 2 . . 31

2.20. Comparativa de desplazamientos en el centro del vano central . . . . . . . . . 32

2.21. Comparativa de aceleración en el centro del vano central . . . . . . . . . . . 33

3.1. Alternativas estudiadas. Ülker Kaustell et al. [12] . . . . . . . . . . . . . . . . . 36

3.2. Valores máximos de aceleración. Ülker Kaustell et al. [12] . . . . . . . . . . . . 37 
3.3. Discretización de la estructura. Romero et al. [33] . . . . . . . . . . . . . . . . . 38

3.4. Modelo del bogie. Romero et al. [33] . . . . . . . . . . . . . . . . . . . . 38

3.5. Serie temporal de aceleración en centro de vano para un tren viajando a (a) $\mathrm{v}=110.4 \mathrm{~m} / \mathrm{s},(\mathrm{b}) \mathrm{v}=103 \mathrm{~m} / \mathrm{s},(\mathrm{c}) \mathrm{v}=95 \mathrm{~m} / \mathrm{s} \mathrm{y} \mathrm{(d)} \mathrm{v}=92.5 \mathrm{~m} / \mathrm{s}$ en (a) terreno infinitamente rígido, (b) terreno duro, (c) terreno de dureza media y (d) terreno blando. Romero et al. [33] . . . . . . . . . . . . . . . . . . . .

3.6. Modelo de elementos finitos y de contorno. Doménech et al. [4] y MartínezRodrigo et al. [15] . . . . . . . . . . . . . . . . . . . . . . . 40

3.7. Resultados de aceleración. Doménech et al. [4] . . . . . . . . . . . . . . . . 41

3.8. Resultados de aceleración. Martínez-Rodrigo et al. [15] . . . . . . . . . . . . 42

3.9. Diferentes modelos de terreno considerados. (a) Semiespacio homogéneo y (b) estrato homogéneo sobre roca. Zangeneh et al. [40] . . . . . . . . . . . . . . 43

3.10. (a) Rigidez dinámica normalizada y (b) coeficiente de amortiguamiento vertical normalizado para la superficie estudiada $\left(3.5 \times 7 \mathrm{~m}^{2}\right)$, restante en estrato homogéneo con velocidad de onda $V_{s}=300 \mathrm{~m} / \mathrm{s}$ y gran $\mathrm{H}$ sobre roca. El eje de abcisas representa la relación entre la frecuencia estudiada y la de resonancia. Zangeneh et al. [40] . . . . . . . . . . . . . . . . . . . . . 43 43

3.11. Generación de rigidez en el pilote en direcciones individuales. Novak et al. [28] 46

3.12. Ejemplo de variación de la rigidez de la pila en función de la frecuencia y la rigidez del terreno. Nogami and Novak [18] . . . . . . . . . . . . . . . . . . . 48

3.13. Efecto de la relación entre las masas del pilote y el terreno en los parámetros de rigidez y amortiguamiento. Novak and Aboul-Ella [21] . . . . . . . . . .

3.14. Parámetros de rigidez y amortiguamiento para vibración horizontal de pilotes con $l / R>25$ en perfiles homogéneos y $l / R>30$ en suelos de perfil parabólico. Novak et al. [28] . . . . . . . . . . . . . . . . . . . . . 50

3.15. Valores de $f_{v 1}$ y $f_{v 2}$ para pilotes empotrados. Novak et al. [28] . . . . . . . 51

3.16. Valores de $f_{v 1}$ y $f_{v 2}$ para pilotes flotantes. Novak et al. [28] . . . . . . . . 52

4.1. Planta. Pérgola Bobadilla II _ . . . . . . . . . . . . . . . . 55

4.2. Tipología de vigas. Pérgola Bobadilla II . . . . . . . . . . . . . . 56

4.3. Tipología de cimentación. Pérgola de Bobadilla II . . . . . . . . . . . . . . . 57

4.4. Modelo en SAP2000. Pérgola de Bobadilla II . . . . . . . . . . . . . . . . . . . 57

4.5. Sección transversal de la vía. Pérgola de Bobadilla II . . . . . . . . . . . . . 58

4.6. Detalle de la conexión entre las vigas y la losa. Pérgola de Bobadilla II _ . . . 60

4.7. Detalle del sistema muelle - amortiguador de un grado de libertad. Pérgola de Bobadilla II . . . . . . . . . . . . . . . . . . . . . . . . . . . 61 
4.8. Puntos de posproceso de valores de aceleración. Pérgola de Bobadilla II . . 62

4.9. Puntos de posproceso de valores de desplazamiento. Pérgola de Bobadilla II . 63

4.10. Valores de $f_{v 1}$ y $f_{v 2}$ para $\frac{l}{R}=50$. Novak et al. [28] . . . . . . . . . . . 64

4.11. Valores de $f_{v 1}$. Pérgola de Bobadilla II . . . . . . . . . . . . . 65

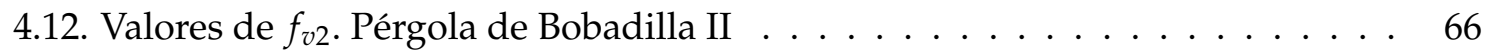

4.13. Valores de $k_{v}$. Pérgola de Bobadilla II . . . . . . . . . . . . . . . 67

4.14. Valores de $k_{v}$. Pérgola de Bobadilla II . . . . . . . . . . . . . . . . . . . . . 68

4.15. Modo 1 de vibración. Pérgola de Bobadilla II . . . . . . . . . . . . . . 72

4.16. Modo 2 de vibración. Pérgola de Bobadilla II _ . . . . . . . . . . . . . . . . 72

4.17. Modo 3 de vibración. Pérgola de Bobadilla II . . . . . . . . . . . . . . . . 73

4.18. Envolvente de aceleración en el punto 2 en estructura con apoyos. Pérgola de Bobadilla II . . . . . . . . . . . . . . . . . . . . . . . . . . . . 74

4.19. Envolvente de desplazamiento en el punto 11 en estructura con apoyos. Pérgola de Bobadilla II . . . . . . . . . . . . . . . . . . . . . . 75

4.20. Envolvente de aceleración en el punto 2 para $E_{p} / G_{s}=250$. Pérgola de Bobadilla II . . . . . . . . . . . . . . . . . . . . . . . . . . . . . . 79

4.21. Envolvente de desplazamiento en el punto 11 para $E_{p} / G_{s}=250$. Pérgola de Bobadilla II . . . . . . . . . . . . . . . . . . . . . . . . . . . . . . 80

4.22. Envolvente de aceleración en el punto 2 para $E_{p} / G_{s}=500$. Pérgola de Bobadilla II . . . . . . . . . . . . . . . . . . . . . . . . 85

4.23. Envolvente de desplazamiento en el punto 11 para $E_{p} / G_{s}=500$. Pérgola de Bobadilla II . . . . . . . . . . . . . . . . . . . . . . . 86

4.24. Envolvente de aceleración en el punto 5 para $E_{p} / G_{s}=1000$. Pérgola de Bobadilla II . . . . . . . . . . . . . . . . . . . . . . . .

4.25. Envolvente de desplazamiento en el punto 11 para $E_{p} / G_{s}=1000$. Pérgola de Bobadilla II . . . . . . . . . . . . . . . . . . . . . . . . . . . . . . . . 92

4.26. Envolvente de aceleración en el punto 5 para $E_{p} / G_{s}=2500$. Pérgola de Bobadilla II . . . . . . . . . . . . . . . . . . . . . . . . . . . . . . . . 97

4.27. Envolvente de desplazamiento en el punto 11 para $E_{p} / G_{s}=2500$. Pérgola de Bobadilla II . . . . . . . . . . . . . . . . . . . . . . . . . . . . . 98

4.28. Envolvente de aceleración en el punto 1 para $E_{p} / G_{s}=5000$. Pérgola de Bobadilla II . . . . . . . . . . . . . . . . . . . . . . . . . . . . . . 103

4.29. Envolvente de desplazamiento en el punto 11 para $E_{p} / G_{s}=5000$. Pérgola de Bobadilla II . . . . . . . . . . . . . . . . . . . . . . . . . . . . . . . . . . . . 104 
4.30. Envolvente de aceleración en el punto 2 para $E_{p} / G_{s}=10000$. Pérgola de Bobadilla II . . . . . . . . . . . . . . . . . . . . . . . . . . . . . . . . . 109

4.31. Envolvente de desplazamiento en el punto 11 para $E_{p} / G_{s}=10000$. Pérgola de Bobadilla II . . . . . . . . . . . . . . . . . . . . . . . . . . . . . . . . 110

4.32. Aceleración máxima en función de $E_{p} / G_{s}$. Pérgola de Bobadilla II . . . . . . . 111 4.33. Desplazamiento máxima en función de $E_{p} / G_{s}$. Pérgola de Bobadilla II . . . 112 


\section{CAPÍtulo 1}

\section{Introducción, objetivos y metodología}

\subsection{Introducción}

El ferrocarril nació en Inglaterra en el siglo XIX como sustitución de la tracción animal en las vagonetas utilizadas sobre carriles de hierro en los trabajos mineros. Tras el invento de la máquina de vapor Stephenson la combinó con los carriles metálicos para diseñar una locomotora que remolcaba un conjunto de vagones sobre los elementos metálicos. La primera línea de ferrocarril existente fue la que unía la ciudad minera de Stockton y el puerto fluvial de Darlington, un tramo de $25 \mathrm{~km}$ construido en 1825.

La primera línea de ferrocarril española se construye en Cuba, uniendo las localidades de La Habana y Bejucal mediante un tramo de $28 \mathrm{~km}$ destinado al transporte de la caña de azúcar. Para presenciar la primera línea de ferrocarril en territorio peninsular hay que remontarse hasta el año 1848, cuando se inaugura la línea entre Barcelona y Mataró.

Las facilidades dadas al capital extranjero para la creación de líneas de ferrocarril hacen que las compañías privadas proliferen, lo que conlleva un gran impulso para la red ferroviaria española. En 1865, solo 17 años después de la inauguración del primer tren peninsular, la longitud de la red española ya alcanzaba los 5000 kilómetros. Y a finales del siglo XIX la longitud era de $10000 \mathrm{~km}$, un $90 \%$ de la existente en 1940. Lo que indica que el recorrido de la red ferroviaria tradicional existente en España en la actualidad data de finales del siglo XIX.

En 1992 se produce un paso al frente en España con la inauguración de la primera línea de alta velocidad en territorio nacional, la que une las ciudades de Madrid y Sevilla. A la construcción de esta línea la siguieron la de otras tantas, lo que ha hecho que España, tras China, sea el segundo país del mundo con la red de alta velocidad más extensa.

La creación de las líneas de alta velocidad confronta fuertemente con la red ferroviaria tradicional existente, que como se ha comentado data principalmente del siglo XIX. Este conflicto ha provocado que muchas líneas de nueva creación deban discurrir por trazados paralelos y prácticamente coincidentes con los ya existentes, dadas las limitaciones de trazado que conlleva la alta velocidad, lo que ha generado graves inconvenientes en la construcción de las mismas. 
Uno de los problemas ocasionados es la aparición de cruces entre las nuevas vías y las existentes con grandes ángulos de esviaje. Estos cruces provocan que la luz a salvar sea de grandes dimensiones. Si se quisiera optar por viaductos tradicionales habría grandes limitaciones en su construcción, ya que la imposibilidad de colocar pilas sobre la vía de ferrocarril a cruzar acarrearía la utilización de vigas de gran canto, con todas las complicaciones que eso conlleva, lo que convierte a la pérgola en la solución óptima para salvar el obstáculo.

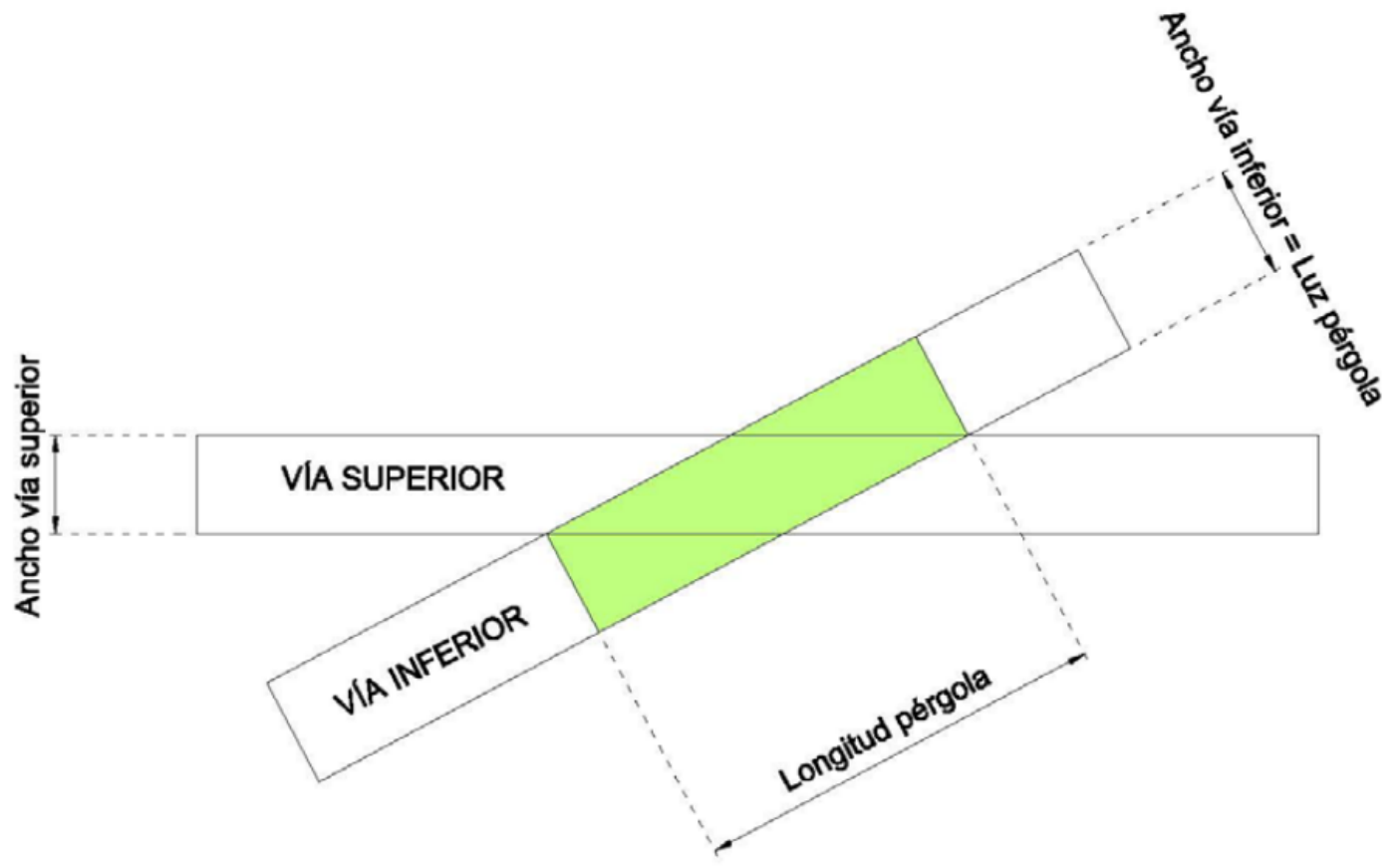

Figura 1.1: Esquema de una pérgola ferroviaria. Ugarte González [37]

Las pérgolas son estructuras singulares ya que existen pocos estudios rigurosos sobre qué modelos emplear y modos de fallos y en la normativa IAPF-2011 tampoco se plantean modelos para su análisis. Sin embargo en los últimos años se han publicado trabajos que han tratado de caracterizar la respuesta dinámica de este tipo de estructuras bajo la circulación de trenes de alta velocidad como Ugarte González [37] o Maldonado Salguero [13].

\subsection{Objetivos}

Los objetivos del presente trabajo son:

- Caracterización de pérgolas mediante modelos que incluyan la cimentación de las mismas y establecimiento de una metodología general para su análisis.

- Desarrollo de una metodología que incluya la interacción suelo - estructura para realizar el análisis dinámico de pérgolas para el paso del ferrocarril de alta velocidad mediante el método semianalítico, una vez se han demostrado las ventajas del mismo.

- Análisis de sensibilidad de respuestas dinámicas en función de la variación de la rigidez del terreno. 


\subsection{Metodología}

La metodología seguida en la elaboración del presente trabajo es:

1. Verificación del método semianalítico para realizar los cálculos dinámicos en estructuras tipo pérgola, tras demostrar sus ventajas y calibrar el método mediante dos modelos bidimensionales realizado mediante una viga de Bernouilli-Euler. El primero una viga de tipología biapoyada y el segundo una viga continua de tres vanos.

2. Revisión del estado del arte en soluciones a la interacción suelo - estructura haciendo especial hincapié en las cimentaciones profundas mediante pilotes y caracterización de la tipología dde rigidez y amortiguamiento presentes en los mismos.

3. Solución a la ecuación dinámica que gobierna el movimiento de la pérgola de manera que sea posible obtener los valores de amortiguamiento geométrico producidos por la interacción suelo - estructura.

4. Concepción y verificación del modelo de pérgola cimentado mediante pilotes empleando el software SAP2000 para introducir la geometría y realizar el análisis modal, previo a la integración analítica en el tiempo.

5. Análisis dinámico del modelo de pérgola empleando el método semianalítico bajo distintos supuestos de rigidez del terreno.

6. Análisis de la variación de las respuestas dinámicas de la pérgola bajo distintos supuestos de rigidez del terreno.

7. Conclusiones de los resultados dinámicos obtenidos y aportaciones futuras al campo del amortiguamiento geométrico en cimentaciones de pérgolas de ferrocarril. 



\section{CAPÍtulo 2}

\section{Método semianalítico}

\subsection{Introducción al método}

La solución semianalítica al paso de cargas móviles fue desarrollada por Martínez Castro et al. [14], profesores de la Universidad de Granada.

El propósito del método es analizar las vibraciones de la viga en el plano vertical, que es el definido por la directriz de la misma y la dirección de las cargas actuantes sobre ella. La viga es dividida mediante nodos. Cada nodo tiene dos grados de libertad, el desplazamiento vertical y la rotación. A continuación se calculan la formas modales y las fecuencias naturales de la estructura mediante el sistema de general de valores y vectores propios. Finalmente las cargas modales equivalentes se expresan analíticamente en función de las formas modales previamente calculadas. A partir de esto se obtiene la expresión matemática de la solución en el dominio del tiempo para cada modo de manera directa.

En este apartado se va a exponer la formulación del método y se va a proceder a validarlo mediante dos ejemplos, una viga biapoyada y una viga continua de tres vanos, con el fin de demostrar que su utilización es válida para el cálculo dinámica de las pérgolas ferroviarias.

\subsection{Ecuación de Gobierno para una viga.}

Se considera una viga de sección variable, sometida a una carga $p_{0}$ que experimente un desplazamiento a través deñ eje longitudinal de la viga, $\mathrm{x}$, a una velocidad de valor constante $v$ : 

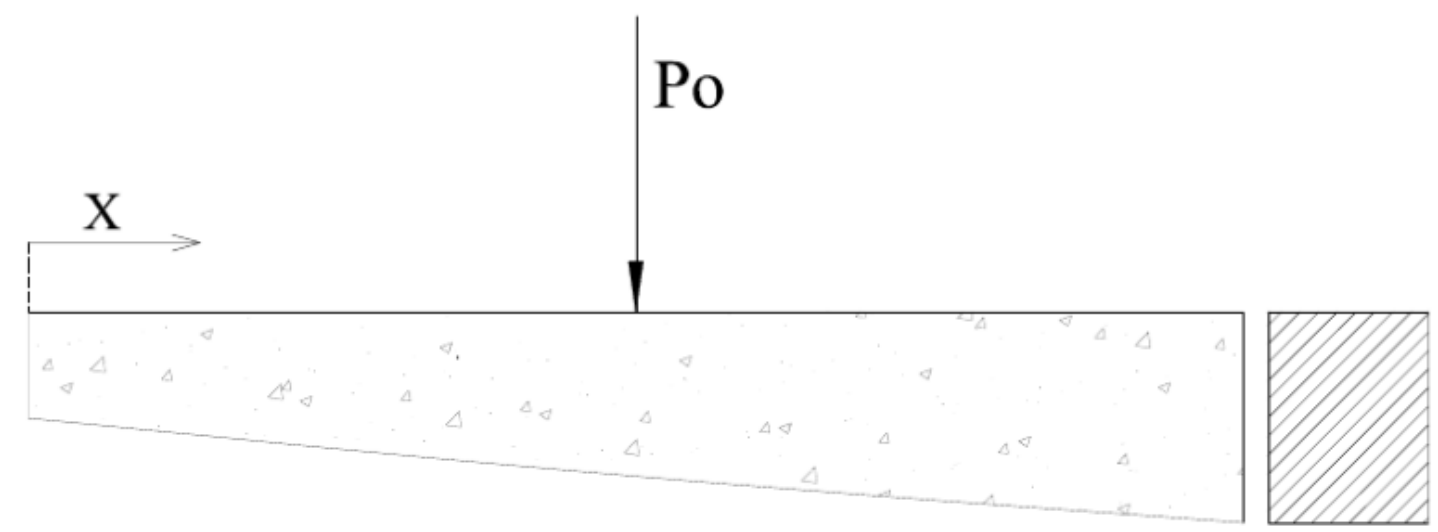

$\mathrm{A}(\mathrm{x})$

\section{$\mathrm{I}(\mathrm{x})$}

Figura 2.1: Esquema de carga móvil sobre viga de sección variable. García Macías [5]

Si se plantea el equilibrio en la rebanada mediante el principio de D'Alembert, despreciando en un principio la contribución del amortiguamiento, se obtiene lo que se indica a continuación:

- Equilibrio de fuerzas:

$$
-\frac{\partial Q(x, t)}{\partial x}-p_{o} \cdot \delta(x-v t)=\rho(x) \cdot \frac{\partial^{2} u}{\partial t^{2}}
$$

Siendo $\delta$ la función Delta de Dirac para imponer la distribución puntual de la carga aplicada con velocidad $v, x$ la coordenada curvilínea de la línea de carga $(x \ni[0, L])$ y $\rho(x)$ la densidad lineal $\left[\frac{M}{L}\right]$.

- Equilibrio de momentos:

$$
\partial M(x, t)=Q(x, t) \partial x \Rightarrow \frac{\partial M(x, t)}{\partial x}=Q(x, t)
$$

Si se sustituye 2.2 en 2.1:

$$
\begin{gathered}
-\frac{\partial\left(\frac{\partial M(x, t)}{\partial x}\right)}{\partial x}-p_{0} \cdot \delta(x-v t)=\rho(x) \cdot \frac{\partial^{2} u}{\partial t^{2}} \\
\frac{\partial^{2} M(x, t)}{\partial x^{2}}-p_{0} \cdot \delta(x-v t)=\rho(x) \cdot \frac{\partial^{2} u}{\partial t^{2}}
\end{gathered}
$$

Como ecuación constitutiva se utiliza como base la ecuación de la viga de BernouilliEuler en la que se adopta la relación lineal momento-curvatura: 


$$
\begin{gathered}
\frac{\frac{\partial^{2} u(x, t)}{\partial x^{2}}}{1+\left(\frac{\partial u}{\partial x}\right)^{2}} \approx \frac{\partial^{2} u(x, t)}{\partial x^{2}}=\frac{M(x, t)}{E I(x)} \\
M(x, t)=E I(x) \cdot \frac{\partial^{2} u(x, t)}{\partial x^{2}}
\end{gathered}
$$

Ecuación que una vez sustituida en 2.4 permite obtener la ecuación que gobierna el problema:

$$
\begin{gathered}
\frac{\partial^{2} E I(x) \cdot \frac{\partial^{2} u(x, t)}{\partial x^{2}}}{\partial x^{2}}-p_{o} \cdot \delta(x-v t)=\rho(x) \cdot \frac{\partial^{2} u}{\partial t^{2}} \\
\rho(x) \cdot \frac{\partial^{2} u}{\partial t^{2}}+\frac{\partial^{2}}{\partial x^{2}}\left[E I(x) \cdot \frac{\partial^{2} u(x, t)}{\partial x^{2}}\right]+p_{o} \cdot \delta(x-v t)=0
\end{gathered}
$$

Ecuación diferencial que se debe ser resuelta supuestas unas condiciones iniciales de reposo:

$$
\begin{gathered}
u(s, 0)=0, \forall x \\
\left.\frac{\partial u(x, t)}{\partial t}\right|_{t=0}=0, \forall x
\end{gathered}
$$

\subsection{Discretización espacial}

Dado un elemento de la línea de carga de longitud L, figura 2.2:

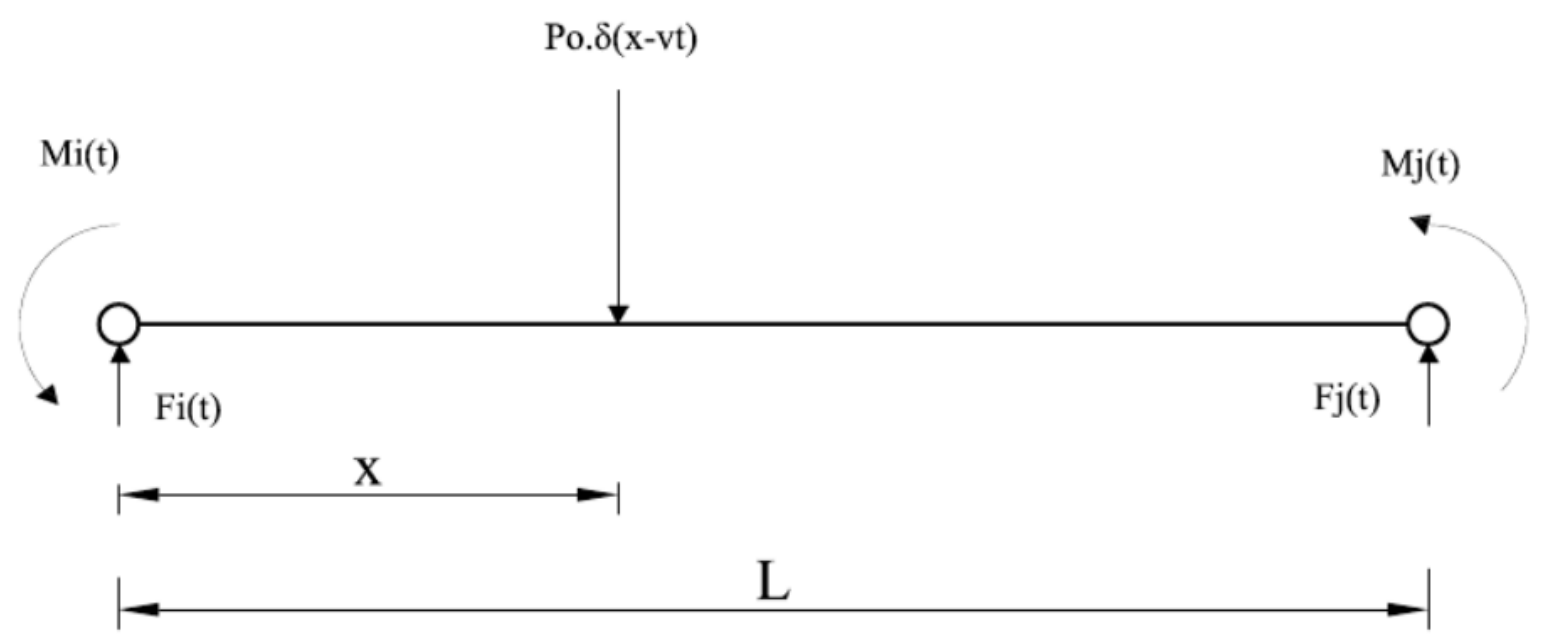

Figura 2.2: Discretización. García Macías [5]

- Fuerzas externas.

1. Fuerzas y momentos nodales:

Si se tiene en consideración el criterio de signos representado en la figura: 


$$
\begin{gathered}
F_{i}^{b}=Q(0, t) \\
F_{j}^{b}=-Q(L, t) \\
M_{i}^{b}=-M(0, t) \\
M_{j}^{b}=M(L, t)
\end{gathered}
$$

\section{Fuerza puntual:}

$$
p_{0} \cdot \delta(x-v t)
$$

3. Fuerza de inercia:

$$
\rho(x) \cdot \frac{\left.\partial^{2} u_{(} x, t\right)}{\partial t^{2}}
$$

- Esfuerzos.

1. Cortantes:

$$
\frac{\partial Q(x, t)}{\partial x}=-p_{0} \cdot \delta(x-v t)-\rho(x) \cdot \frac{\partial^{2} u(x, t)}{\partial t^{2}}
$$

Que integrándola entre 0 y $x \in[0, L]$ :

$$
Q(x, t)=Q(0, t)-\int_{0}^{x} p_{0} \cdot \xi(\xi-v t) d \xi-\int_{0}^{x} \rho(\xi) \cdot \frac{\partial^{2} u(\xi, t)}{\partial t^{2}} d \xi
$$

2. Flectores:

$$
\begin{gathered}
\frac{\partial M(x, t)}{\partial x}=Q(x, t) \cdot d x \\
M(x, t)=M(0, t)+\int_{0}^{x} Q(\xi, t) d \xi
\end{gathered}
$$

En este punto, aplicando los conceptos de elementos finitos, se procede a trabajar con la formulación débil de la ecuación de Bernouilli-Euler de la viga multiplicando por una función de ponderación espacial, $u^{*}(x)$, y aplicando el método de integración por partes:

Se procede a utilizar la siguiente notación:

$$
\begin{aligned}
& \frac{\partial u(x, t)}{\partial x}=\dot{u}(x, t) \\
& \frac{\partial u(x, t)}{\partial t}=\dot{u}(x, t)
\end{aligned}
$$

Si se multiplica 2.8 por $u^{*}(x)$ :

$$
\begin{gathered}
\rho(x) \cdot \ddot{u}(x, t)+\left(u^{\prime \prime}(x, t) \cdot E I(x)\right)^{\prime \prime}=-p_{0} \cdot \delta(x-v t) \\
\rho(x) \cdot \ddot{u}(x, t) \cdot u^{*}(x)+\left(u^{\prime \prime}(x, t) \cdot E I(x)\right)^{\prime \prime} \cdot u^{*}(x)=-p_{0} \cdot \delta(x-v t) \cdot u^{*}(x)
\end{gathered}
$$

Integrando en el dominio: 


$$
\begin{aligned}
& \int_{0}^{L} \rho(x) \cdot \ddot{u}(x, t) \cdot u^{*}(x) d x+\int_{0}^{L}\left(u^{\prime \prime}(x, t) \cdot E I(x)\right)^{\prime \prime} \cdot u^{*}(x) d s= \\
= & -\int_{0}^{L} p_{0} \cdot \delta(x-v t) \cdot u^{*}(x) d x
\end{aligned}
$$

Es posible aplicar doble integración por partes en el segundo sumando del primer término:

$$
\begin{aligned}
& \int_{0}^{L}\left(u^{\prime \prime}(x, t) \cdot E I(x)\right)^{\prime \prime} \cdot u^{*}(x) d x= \\
= & \left.u^{*}(x) \cdot\left(u^{\prime \prime}(x, t) \cdot E I(x)\right)^{\prime}\right|_{0} ^{L}-\int_{0}^{L}\left(u^{\prime \prime}(x, t) \cdot E I(x)\right)^{\prime} \cdot \frac{\partial u^{*}(x)}{\partial x} d s= \\
= & \left.u^{*}(x) \cdot\left(u^{\prime \prime}(x, t) \cdot E I(x)\right)^{\prime}\right|_{0} ^{L}-\left.\left(u^{\prime \prime}(x, t) \cdot E I(x)\right) \cdot \frac{\partial u^{*}(x)}{\partial x}\right|_{0} ^{L}+ \\
+ & \int_{0}^{L} u^{\prime \prime}(x, t) \cdot E I(x) \cdot \frac{\partial^{2} u^{*}(x)}{\partial x^{2}} d x
\end{aligned}
$$

Analizando detenidamente los sumandos de la expresión anterior es posible identificar fácilmente(Por la relación Momento-Curvatura de Euler-Bernouilli):

$$
\begin{aligned}
& Q(x, t)=\left(u^{\prime \prime} \cdot E I(x)\right)^{\prime} \\
& M(x, t)=u^{\prime \prime} \cdot E I(x)
\end{aligned}
$$

Con lo cual, y teniendo en cuenta las relaciones anteriores, 2.27 y 2.28 :

$$
\begin{gathered}
\left.\left(u^{\prime \prime} \cdot E I(x)\right)^{\prime} \cdot u^{*}(x)\right|_{0} ^{L}=\left.Q(L, t) \cdot u^{*}(x)\right|_{0} ^{L}= \\
=Q(L, t) \cdot u^{*}(L)-Q(0, t) \cdot u^{*}(0)=-F_{j}^{b} \cdot u^{*}(L)-F_{i}^{b} \cdot u^{*}(0) \\
\left.u^{\prime \prime} \cdot E I(s) \cdot \frac{\partial \cdot u^{*}(s)}{\partial s}\right|_{0} ^{L}=\left.M(s) \cdot \frac{\partial u^{*}}{\partial s}\right|_{0} ^{L}= \\
=-\left.M_{j}^{b} \cdot \frac{\partial u^{*}}{\partial s}\right|_{L}-\left.M_{i}^{b} \cdot \frac{\partial u^{*}}{\partial s}\right|_{0}
\end{gathered}
$$

Con lo que la ecuación 2.26 resultaría: 


$$
\begin{aligned}
& \int_{0}^{L}\left(u^{\prime \prime}(s, t) \cdot E I(s)\right)^{\prime \prime} \cdot u^{*}(s) d x= \\
& \int_{0}^{L} u^{\prime \prime}(s, t) \cdot E I(s) \cdot \frac{\partial^{2} u^{*}(s)}{\partial s^{2}}-F_{j}^{b} \cdot u^{*}(L)-F_{i}^{b} \cdot u^{*}(0)-\left.M_{j}^{b} \cdot \frac{\partial u^{*}}{\partial s}\right|_{L}+ \\
& +\left.M_{i}^{b} \cdot \frac{\partial u^{*}}{\partial s}\right|_{0}
\end{aligned}
$$

En cuanto al segundo miembro de la ecuación 2.25:

$$
\int_{0}^{L} p_{0} \cdot \delta(x-v t) \cdot u^{*}(x) \cdot d x=p_{0} \cdot u^{*}(v t)
$$

Teniendo en cuenta las propiedades de la función Delta de Dirac.

Por lo tanto, si se sustituyen las ecuaciones 2.31 y 2.32 en la ecuación 2.25 se obtiene lo siguiente:

$$
\begin{aligned}
& \int_{0}^{L} \rho(x) \cdot \ddot{u}(x, t) \cdot u^{*}(x) d x+\int_{0}^{L}\left(u^{\prime \prime}(x, t) \cdot E I(x)\right)^{\prime \prime} \cdot u^{*}(s) d x= \\
= & +F_{j}^{b} \cdot u^{*}(L)+F_{i}^{b} \cdot u^{*}(0)+\left.M_{j}^{b} \cdot \frac{\partial u^{*}}{\partial x}\right|_{L}+\left.M_{i}^{b} \cdot \frac{\partial u^{*}}{\partial x}\right|_{0}-p_{o} \cdot u^{*}(v t)
\end{aligned}
$$

Expresión de la que no se ha impuesto condición alguna sobre $u^{*}(x)$ más que sea continua y derivable. Por ello, y continuando con los conceptos básicos de elementos finitos, se considera una base de los polinomios de Hermite de grado menor o igual a 3, pues se discretizarán los elementos barra por dos nodos extremos con 4 grados de libertad, dos de desplazamientos y otros dos de giro.

Se supondrá que $\mathrm{u}(\mathrm{x}, \mathrm{t})$ del elemento puede representarse de forma exacta a través de una combinación lineal de la base de los polinomios anteriormente indicada.

Por ello:

$$
u(x, t)=\sum_{n=1}^{4} y_{n}(t) \cdot h_{n}(x)
$$

Siendo las expresiones de los polinomios de Hermite:

$$
\begin{gathered}
{\left[U_{i}\right] \rightarrow h_{1}(x)=1-3 \cdot\left(\frac{x}{L}\right)^{2}+2 \cdot\left(\frac{x}{L}\right)^{3}} \\
{\left[\theta_{i}\right] \rightarrow h_{2}(x)=L \cdot\left[\frac{x}{L}-2 \cdot\left(\frac{x}{L}\right)^{2}+\left(\frac{x}{L}\right)^{3}\right]} \\
{\left[U_{j}\right] \rightarrow h_{3}(x)=3 \cdot\left(\frac{x}{L}\right)^{2}-2 \cdot\left(\frac{x}{L}\right)^{3}}
\end{gathered}
$$




$$
\left[\theta_{j}\right] \rightarrow h_{4}(x)=L \cdot\left[-\left(\frac{x}{L}\right)^{2}+\left(\frac{x}{L}\right)^{3}\right]
$$

Si se desarrolla la ecuación 2.34 usando las relaciones anteriores:

$$
u(x, t)=y_{i} \cdot h_{1}(x)+\theta_{i} \cdot h_{2}(x)+y_{j} \cdot h_{3}(x)+\theta_{j} \cdot h_{4}(x)
$$

De modo que es posible obtener la velocidad y la aceleración:

$$
\begin{gathered}
u(\dot{x}, t)=\dot{y}_{i} \cdot h_{1}(x)+\dot{\theta}_{i} \cdot h_{2}(x)+\dot{y}_{j} \cdot h_{3}(x)+\dot{\theta}_{j} \cdot h_{4}(x) \\
u(\dot{x}, t)=\sum_{n=1}^{4} y_{n}(t) \cdot h_{n}(x) \\
u(\ddot{x}, t)=\ddot{y}_{i} \cdot h_{1}(x)+\ddot{\theta}_{i} \cdot h_{2}(x)+\ddot{y}_{j} \cdot h_{3}(x)+\ddot{\theta}_{j} \cdot h_{4}(x) \\
u(\ddot{x}, t)=\sum_{n=1}^{4} y_{n} \ddot{n}(t) \cdot h_{n}(x)
\end{gathered}
$$

Si además se aceptan como funciones de ponderación $u^{*}(x)$ las propias funciones de Hermite:

$$
\begin{aligned}
& \int_{0}^{L} \rho(x) \cdot \sum_{n=1}^{4}\left[y_{n}(t) \cdot h_{n}(x)\right] \cdot h m(x) d x+\int_{0}^{L} \sum_{n=1}^{\ddot{4}}\left[y_{n}(t) \cdot \frac{\partial^{2} h_{n}(x)}{\partial x^{2}}\right] \cdot \operatorname{EI}(x) h_{m}(x) \partial x^{2}= \\
= & +F_{j}^{b} \cdot h_{m}(L)+F_{i}^{b} \cdot h_{m}(0)+\left.M_{j}^{b} \cdot \frac{\partial h_{m}(x)}{\partial x}\right|_{L}+\left.M_{i}^{b} \cdot \frac{\partial h_{m}(x)}{\partial x}\right|_{0}-p_{o} \cdot h_{m}(v t)
\end{aligned}
$$

Con lo cual se tiene la posibilidad de escribir de forma matricial:

$$
\left[\begin{array}{llll}
m_{11} & m_{12} & m_{13} & m_{14} \\
m_{21} & m_{22} & m_{23} & m_{24} \\
m_{31} & m_{32} & m_{33} & m_{34} \\
m_{41} & m_{42} & m_{43} & m_{44}
\end{array}\right]\left[\begin{array}{l}
\ddot{y}_{i}(v t) \\
\ddot{\theta}_{i}(v t) \\
\ddot{y}_{j}(v t) \\
\ddot{\theta}_{j}(v t)
\end{array}\right]+\left[\begin{array}{llll}
k_{11} & k_{12} & k_{13} & k_{14} \\
k_{21} & k_{22} & k_{23} & k_{24} \\
k_{31} & k_{32} & k_{33} & k_{34} \\
k_{41} & k_{42} & k_{43} & k_{44}
\end{array}\right]\left[\begin{array}{c}
y_{i}(v t) \\
\theta_{i}(v t) \\
y_{j}(v t) \\
\theta_{j}(v t)
\end{array}\right]=\left[\begin{array}{c}
F_{i}^{b}(v t) \\
M_{i}^{b}(v t) \\
F_{j}^{b}(v t) \\
M_{j}^{b}(v t)
\end{array}\right]-p_{0}\left[\begin{array}{l}
h_{1}(v t) \\
h_{2}(v t) \\
h_{3}(v t) \\
h_{4}(v t)
\end{array}\right]
$$

Siendo:

$$
\begin{gathered}
M_{i j}=\int_{0}^{L} \rho(x) \cdot h_{i}(x) \cdot h_{j}(x) d x \\
K_{i j}=\int_{0}^{L} h_{i}^{\prime \prime}(x) \cdot E I(x) \cdot h_{j}^{\prime \prime}(x) d x
\end{gathered}
$$

Matrices cuyos subíndices indican que son matrices simétricas. 
Si se procede a acoplar estas matrices elementales es posible obtener una formulación global para la estructura completa:

$$
\mathbf{M} \ddot{\mathbf{y}}+\mathbf{K y}=-p_{0} \mathbf{h}(v t)
$$

\subsection{Resolución}

Se está trabajando con matrices simétricas por lo que la forma más sencilla de resolución es tratar de desacoplar el sistema, para ello se realiza un cambio de base, mediante la matriz $C$, matriz de cambio de base (obtenida del problema de autovalores), del sistema $B$ (que coincidirá con la base de vectores propios) a la base canónica E:

$$
\left(-w^{2} \mathbf{M}+\mathbf{K}\right) \mathbf{y}(t)=0 \Rightarrow\left|\mathbf{K}-w^{2} \mathbf{M}\right|=0
$$

Problema de valores propios que proporciona los vectores propios o modos de vibración que compondrán por columnas la matriz $C$. De este modo, a través de dicha matriz $C$, se establece una relación entre las nuevas coordenadas del vector mediante las expresiones siguientes:

$$
\begin{gathered}
y=C q \\
q=C^{-1} y
\end{gathered}
$$

De modo que si se realiza dicho cambio de base y se multiplica por la izquierda por la transpuesta de $\mathrm{C}$, se obtienen matrices diagonales para $M$ y $K$, consiguiendo desacoplar el sistema:

$$
\mathbf{C}^{T} \mathbf{M C} \ddot{\mathbf{q}}+\mathbf{C}^{T} \mathbf{K C q}=-p_{0} \mathbf{C}^{T} \mathbf{h}(v t)
$$

Las matrices de masa y rigidez resultan diagonales:

$$
\begin{aligned}
\mathbf{M}_{D} & =\mathbf{C}^{T} \mathbf{M C} \\
\mathbf{K}_{D} & =\mathbf{C}^{T} \mathbf{K C}
\end{aligned}
$$

Si multiplica por la izquierda por $\mathbf{M}_{D}^{-1}$ es posible reescribir:

$$
\mathbf{M}_{D}^{-} 1 \cdot \mathbf{M}_{D} \ddot{\mathbf{q}}+\mathbf{M}_{D}^{-} 1 \cdot \mathbf{K}_{D} \cdot \mathbf{q}=-p_{0} \cdot \mathbf{M}_{D}^{-} 1 \cdot \mathbf{C}^{T} \mathbf{h}(v t)
$$




$$
\ddot{\mathbf{q}}+\mathbf{D} \cdot \mathbf{q}=\mathbf{G} \cdot \mathbf{h}(v t)
$$

Siendo D una matriz diagonal tal que $D_{i i}=w_{i}^{2}$, y la matriz G:

$$
\mathbf{G}=-p_{0} \cdot \mathbf{M}_{D}^{-} 1 \cdot \mathbf{C}^{T}
$$

${ }^{*}$ ) Nótese que si se normaliza con la matriz de masa, $M_{D}=\mathrm{I}, \mathrm{y} \mathrm{G}=-p_{0} \cdot \mathrm{C}^{T}$

De este modo se ha conseguido desacoplar el sistema, siendo resoluble para cada función $q_{i}(t)$ independientemente, de modo que se puede expresar:

$$
\ddot{q}_{n}(t)+w_{n}^{2} \cdot q_{n}(t)=\sum_{m=1}^{4} G_{n m} h_{m}(v t)
$$

Llegados a este punto se pueden incluir los efectos producidos por el amortiguamiento:

$$
\ddot{q}_{n}(t)+2 \zeta w_{n} \dot{q}_{n}(t)+w_{n}^{2} \cdot q_{n}(t)=\sum_{m=1}^{4} G_{n m} h_{m}(v t)
$$

En la que $\zeta$ representa la tasa de amortiguamiento, en el que se puede introducir un valor distinto para cada modo (lo que sería más ajustado al comportamiento real ya que es evidente pensar que se amortiguarán de mejor manera los modos altos a los bajos), aunque en la práctica se suele optar por la inclusión de un amortiguamiento constante para cada uno de los modos por lo que en el presente documento se procederá de igual forma.

Por lo tanto, el problema a resolver viene dado por:

$$
\begin{gathered}
\ddot{q}_{n}(t)+2 \zeta w_{n} \dot{q}_{n}(t)+w_{n}^{2} \cdot q_{n}(t)=\sum_{m=1}^{4} G_{n m} h_{m}(v t) \\
\left.q_{i}(t)\right|_{t=0}=q_{i}(0) \\
\left.\dot{q}_{i}(t)\right|_{t=0}=\dot{q}_{i}(0)
\end{gathered}
$$

Se considera una escala de referencia local para cada elemento, en el que el instante $t$ igual a 0 coincicide con el instante en que la carga entra en el elemento, coincidente con el punto de abcisa igual a 0 . De este modo se evitan exponenciales con términos fuera de rango (se puede entender sin complicación si se visualiza la ecuación de un sistema de un grado de libertad amortiguado, en el que se dispone de una función exponencial decreciente con el aumento del tiempo).

Si se observa la ecuación 2.60, se aprecia que se trata de una ecuación diferencial lineal no homogénea por lo que la solución se descompone en la suma de la solución a la ecuación homogénea más una solución particular: 


$$
q(t)=q_{H}(t)+q_{P}(t)
$$

- Solución homogénea:

$$
\begin{gathered}
q_{H}(t)=e^{-\zeta w_{i} t}\left(C_{1} \cos \left(w_{i D}\right) t+C_{2} \sin \left(w_{i D} t\right)\right) \\
w_{i D}=w_{i} \sqrt{1-\zeta^{2}}
\end{gathered}
$$

- Solución particular:

Si se descompone el término no homogéneo:

$$
p_{0}\left(G_{1} h_{1}(v t)+G_{2} h_{2}(v t)+G_{3} h_{3}(v t)+G_{4} h_{4}(v t)\right)
$$

Es más que un polinomio de tercer grado, lo que provoca que la ecuación particular sea de la forma:

$$
q_{P}(t)=\alpha_{0}+\alpha_{1} v t+\alpha_{2}(v t)^{2}+\alpha_{0} 3(v t)^{3}
$$

Ecuación cuyos coeficientes se obtienen sustituyendo en la ecuación 2.60 y comparando coeficientes de mismo grado, obteniéndose:

$$
\begin{gathered}
\alpha_{0}=v^{3} \alpha_{01}+v^{2} \alpha_{02}+v \alpha_{03}+\alpha_{04} \\
\alpha_{1}=v^{2} \alpha_{11}+v \alpha_{12}+\alpha_{13} \\
\alpha_{2}=v \alpha_{21}+\alpha_{22} \\
\alpha_{3}=\alpha_{31}
\end{gathered}
$$

Los diez coeficienes que aparecen obedecen a las siguientes expresiones:

$$
\begin{gathered}
{[1] \mapsto \alpha_{01}=-\frac{24 \zeta\left(2 \zeta^{2}-1\right)\left(2 G_{1}-2 G_{3}+\left(G_{2}+G_{4}\right) L\right)}{L^{3} w_{i}^{5}}} \\
{[2] \mapsto \alpha_{02}=-\frac{2\left(4 \zeta^{2}-1\right)\left(3 G_{1}-3 G_{3}+\left(2 G_{2}+G_{4}\right) L\right)}{L^{2} w_{i}^{4}}} \\
{[3] \mapsto \alpha_{03}=-\frac{2 G_{2} \zeta}{w_{i}^{3}}} \\
{[4] \mapsto \alpha_{04}=\frac{G_{1}}{w_{i}^{2}}}
\end{gathered}
$$




$$
\begin{gathered}
{[5] \mapsto \alpha_{11}=\frac{6\left(4 \zeta^{2}-1\right)\left(2 G_{1}-2 G_{3}+\left(G_{2}+G_{4}\right) L\right)}{L^{3} w_{i}^{4}}} \\
{[6] \mapsto \alpha_{12}=\frac{4 \zeta\left(3 G_{1}-3 G_{3}+\left(2 G_{2}+G_{4}\right) L\right.}{L^{2} w_{i}^{3}}} \\
{[7] \mapsto \alpha_{13}=\frac{G_{2}}{w_{i}^{2}}} \\
{[8] \mapsto \alpha_{21}=-\frac{6 \zeta\left(2 G_{1}-2 G_{3}+\left(G_{2}+G_{4}\right) L\right.}{L^{3} w_{i}^{3}}} \\
{[9] \mapsto \alpha_{22}=-\frac{4 \zeta\left(3 G_{1}-3 G_{3}+\left(2 G_{2}+G_{4}\right) L\right.}{L^{2} w_{i}^{2}}} \\
{[10] \mapsto \alpha_{31}=\frac{2 G_{1}-2 G_{3}+\left(G_{2}+G_{4}\right) L}{L^{3} w_{i}^{2}}}
\end{gathered}
$$

Estos diez elementos dependen del amortiguamiento, de la longitud del elemento, de las frecuencias naturales y de los coeficientes $G_{i}$ que a su vez depende de los modos de vibración, sin depender en ningún momento de la velocidad de paso de la carga por o que se podrían determinar en un principio estos diez parámetros para la malla completa.

En último lugar resta determinar los parámetros $C_{1}$ y $C_{2}$ de la solución homogénea a partir de la imposición de las condiciones iniciales:

$$
\begin{gathered}
q(0)=\alpha_{0}+C_{1} \\
C_{1}=q(0)-\alpha_{0} \\
\dot{q}(0)=-\zeta w_{i} C_{1}+w_{i D} C_{2}+\alpha_{1} v \\
C_{2}=\frac{\dot{q}(0)+\zeta w_{i} C_{1}-\alpha_{1} v}{w_{i D}}
\end{gathered}
$$

\subsection{Generalización del método.}

Se observa en la solución obtenida, 2.67 y 2.64, que todos los parámetros de los que depende $\left(C_{i}, w_{i}\right.$ y $\left.G_{i}\right)$ se obtienen del análisis modal, estando la parte temporal definida de forma analítica. En ningún momento aparecen imposiciones de geometría, sección o inercia, estando contenida toda esta información geométrica en forma de masa e inercia a través del análisis modal.

Se puede interpretar la ecuación matricial 2.45, mediante un operador diferencial L: 


$$
\mathcal{L}\{u(\bar{x}, t), v(\bar{x}, t), w(\bar{x}, t)\}=-p_{0} \cdot \delta(s-v t)
$$

Con $s$ coordenada curvilínea de una línea de carga $C^{1}$. Se resuelve mediante separación de variables, espacial y temporal, a través del análisis modal que, gracias a la diagonalización de las matrices de rigidez y masa, desacopla todo el sistema.

$$
\mathbf{C}^{T} \mathbf{M C} \ddot{\mathbf{q}}+\mathbf{C}^{T} \mathbf{K C q}=-p_{0} \cdot \mathbf{C}^{T} \cdot h(v t)
$$

Por lo tanto, el método semianalítico se encarga de realizar la integración temporal de la información modal, información que le es proporcionada externamente, como en los casos que se analizan en este documento, mediante el software SAP2000, o cualquier otro software de análisis modal al uso. Por ello, parece razonable pensar que esta metodología para vigas es generalizable a líneas de carga genéricas, con la la única imposición de la derivabilidad, $C^{1}$, de la línea de carga, de modo que la discretización espacial mediante los polinomios de Hermite permita una transición suave en su definición evitando la existencia de discontinuidades que introduzcan picos de aceleración ficticios.

En la publicación de Museros et al. [16], en la que los autores desarrollan la aplicación del método a placas de Kirchhoff esviadas, se observa de nuevo la generalidad del método en cuanto no se exige más que la derivabilidad de la línea de carga, tratando el dominio espacial mediante análisis modal de forma independiente.

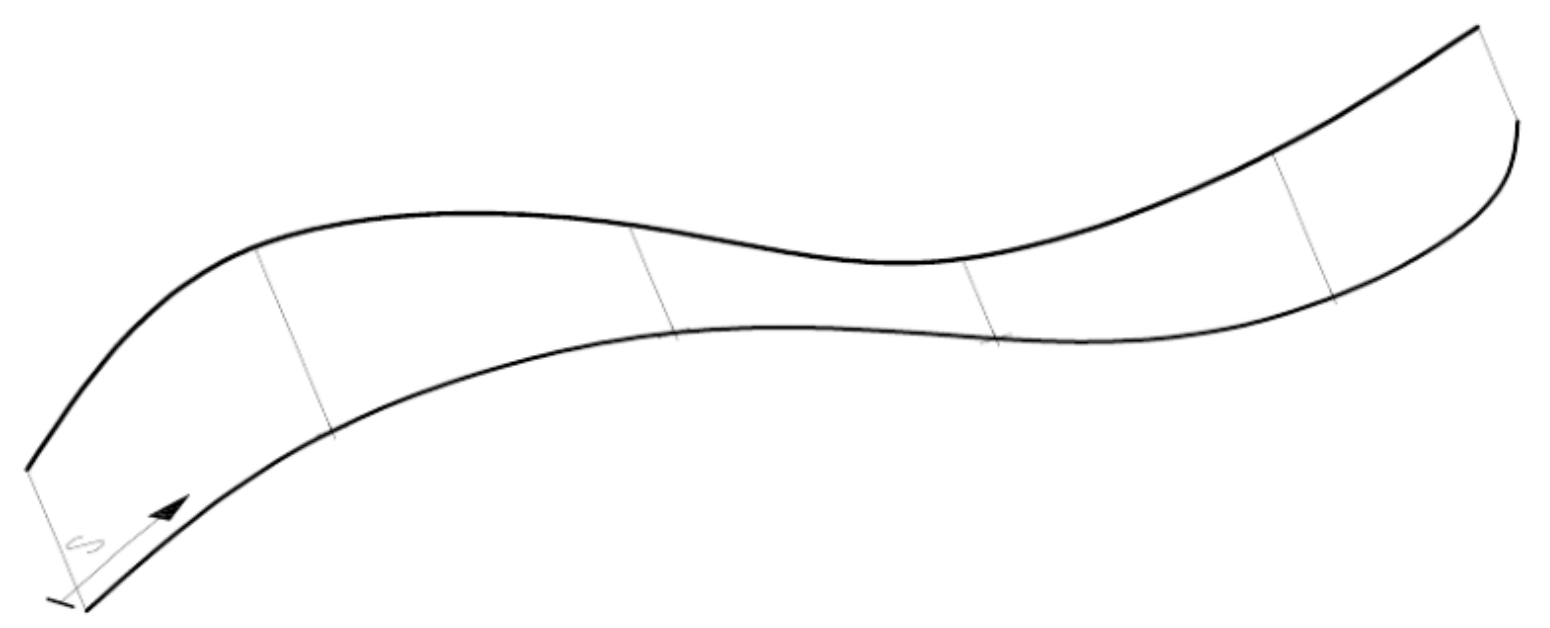

Figura 2.3: Esquema de línea de carga C1. García Macías [5]

Por lo tanto, el esquema de cálculo consiste, en primer lugar, en la determinación de los diez parámetros $\alpha_{n}$, así como las matrices CY G mediante cualquier software de elementos 
finitos y análisis modal, información que recibe el algoritmo semianalítico para, posteriormente, realizar la integración temporal de la solución, quedando así determinada por completo la misma en términos de desplazamientos y giros (solución analítica, no aproximada) de los puntos de postproceso (puntos donde estudiamos aceleraciones y desplazamientos, figura 2.4) que previamente introduzcamos, de una manera sencilla gracias a la diagonalización de las matrices de masa y rigidez con el consecuente desacoplamiento del sistema.

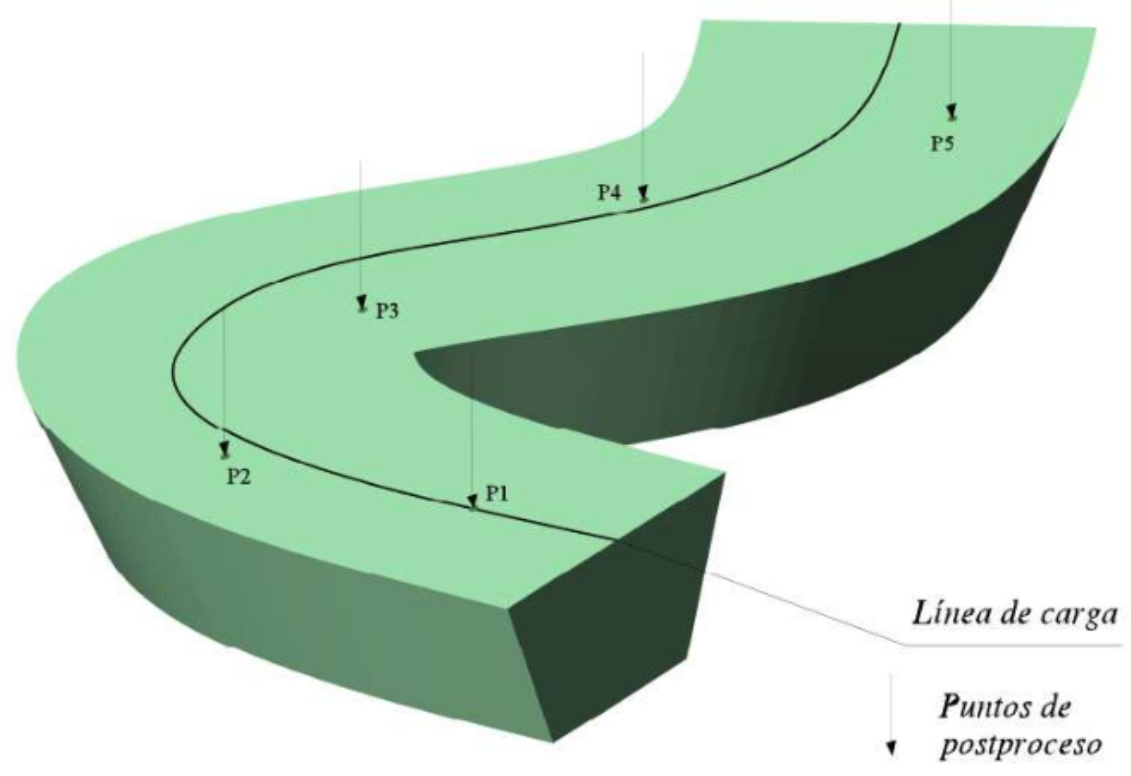

Figura 2.4: Esquema de línea de carga y puntos de postproceso en estructura. García Macías [5]

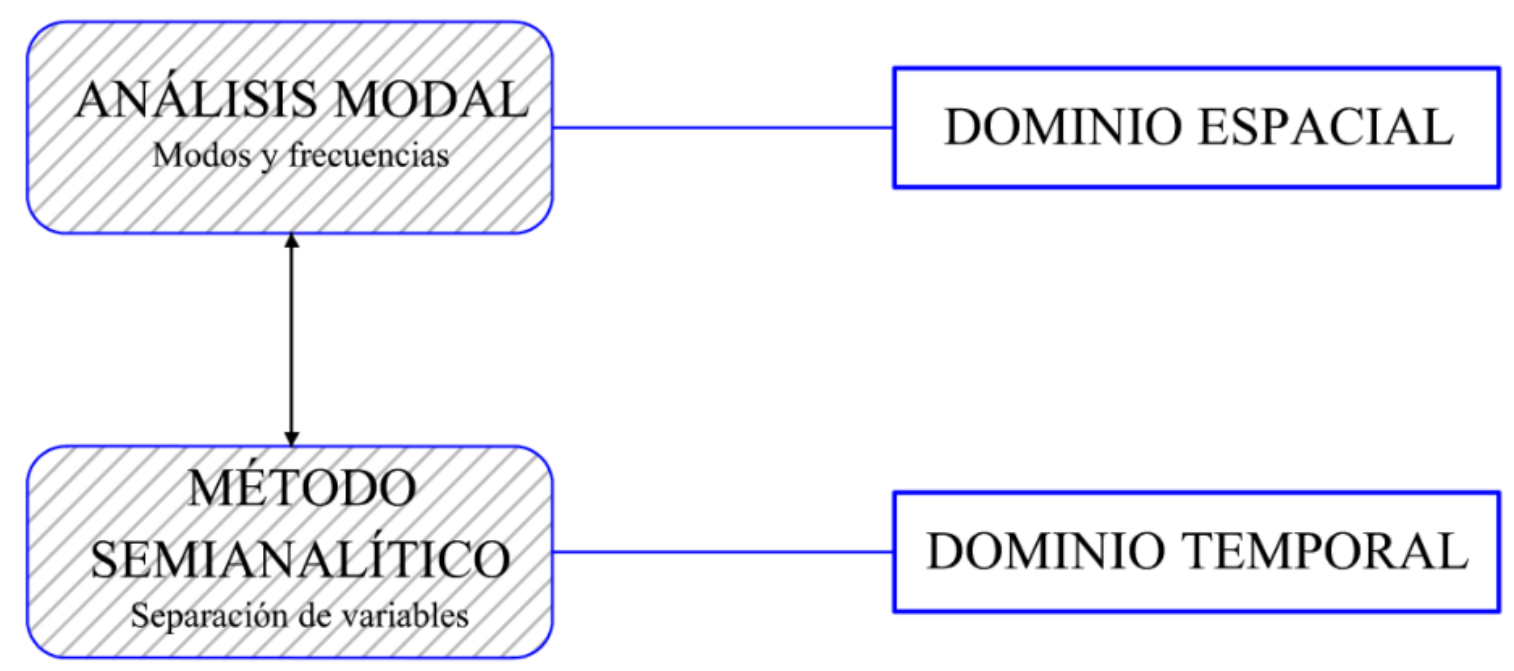

Figura 2.5: Esquema de cálculo del Método Semianalítico Generalizado. García Macías [5] 


\subsection{Pruebas de validación}

\subsubsection{Planteamiento}

Las estructuras que van a ser utilizadas en las pruebas de validación son:

1. Viga isostática biapoyada

2. Viga continua de tres vanos

Sobre las estructuras indicadas se va a realizar un cálculo dinámico con el fin de comprobar los resultados que arrojan tres métodos distintos y comprobar si efectivamente son coincidentes. Los métodos empleados van a ser la solución analítica (solamente en la primera prueba), el cálculo mediante el método semianalítico y el cálculo mediante el programa SAP2000.

El objetivo es el siguiente:

1. Comparativa entre los resultados obtenidos por los diferentes métodos.

2. Detectar las ventajas del método semianalítico frente al cálculo con el programa SAP2000.

3. Demostrar que la aplicación del método semianalítico es válida en el cálculo de pérgolas.

\subsubsection{Prueba de validación 1: Viga de Bernouilli-Euler biapoyada}

Esta primera prueba consiste en la comparativa de resultados dinámicos obtenidos durante el paso de una carga móvil por la viga representada en la figura 2.6.

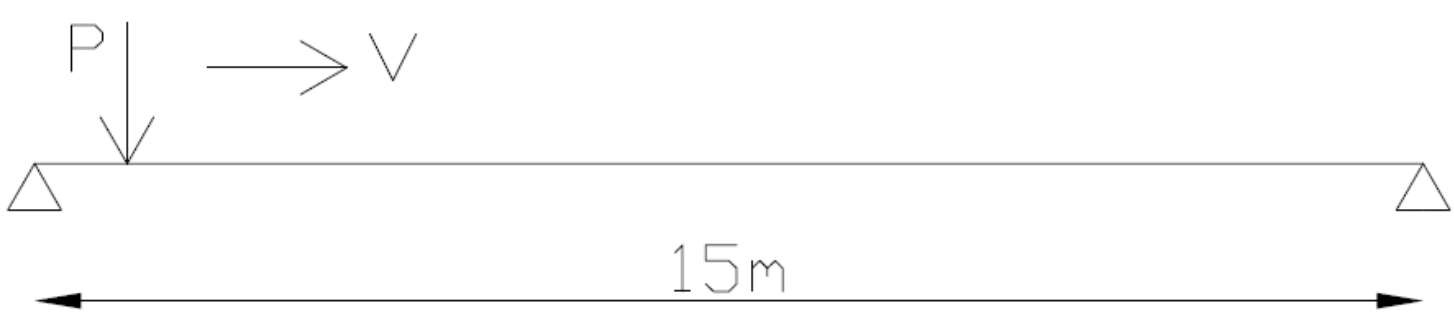

Figura 2.6: Geometría de la viga. Prueba 1.

Las características de la viga son:

- Viga de Bernouilli - Euler

- Longitud: $\mathrm{L}=15 \mathrm{~m}$

- Masa por unidad de longitud: $\rho \mathrm{A}=15.291 \mathrm{t} / \mathrm{m}^{3}$

- Rigidez a flexión: $\mathrm{EI}=7.695 \mathrm{GNm}^{2}$

- Amortiguamiento modal: $\xi=2 \%$

- Velocidad de paso: $\mathrm{V}=100 \mathrm{~m} / \mathrm{s}$

- Carga móvil: P = 195 kN 
Para efectuar el cálculo dinámico se divide la viga de manera uniforme en 8 elementos. Se realiza un análisis bidimensional y el cálculo modal mediante el software SAP2000 considerando 3 modos de vibración.

Los resultados de períodos y frecuencias naturales son los representados en la tabla 2.1.

\begin{tabular}{|c|c|c|}
\hline Modo $\left(\mathrm{n}^{\mathrm{o}}\right)$ & Período $(\mathrm{s})$ & Frecuencia $(\mathrm{Hz})$ \\
\hline 1 & 0.2019 & 4.9524 \\
\hline 2 & 0.0505 & 19.8040 \\
\hline 3 & 0.0225 & 44.4900 \\
\hline
\end{tabular}

Cuadro 2.1: Frecuencias y períodos de vibración. Prueba 1

Una vez obtenidas las formas y frecuencias modales se va a proceder al análisis dinámico de la viga mediante el paso de una carga puntual. El análisis dinámico se va a efectuar a través de los siguientes métodos:

1. Fórmula analítica

2. Programa comercial SAP2000

3. Método semianalítico

\section{Cálculo mediante fórmula analítica}

La solución de esta ecuación es posible mediante el método de separación de variables. Consideraremos que la solución para $\mathrm{w}(\mathrm{x}, \mathrm{t})$ puede expresarse mediante el producto de una función que sólo depende de $\mathrm{x}$, la cual notaremos como $\phi(x)$, por otra función que sólo depende del tiempo $(\mathrm{t})$, la cual notaremos como $\mathrm{q}(\mathrm{t})(2.88)$.

$$
w(x, t)=\phi(x) q(t)
$$

La solución espacial $\phi_{n}(x)$ se denominan modos de vibración y poseen la expresión indicada en la fórmula (2.89).

$$
\phi_{n}(x)=\operatorname{sen}\left(\frac{n \pi x}{l}\right)
$$

La expresión de la solución temporal es la indicada en la fórmula (2.90).

$$
q_{n}(t)=e^{-\xi_{n} \omega_{n} t}\left[A_{n} \cos \left(\omega_{D n} t\right)+B_{n} \operatorname{sen}\left(\omega_{D n} t\right)+\alpha_{n} \cos \left(\Omega_{n} t\right)+\beta_{n} \operatorname{sen}\left(\Omega_{n} t\right)\right]
$$

La expresión de las variables que aparecen en la fórmula (2.90) es el siguiente: 


$$
\begin{gathered}
\alpha_{n}=\frac{4 P_{0} \xi_{n} \omega_{n} \Omega_{n}}{m l^{\prime}} \\
\beta_{n}=\frac{2 P_{0}\left(\Omega_{n}^{2}-\omega_{n}^{2}\right)}{m l^{\prime}} \\
A_{n}=-\alpha_{n} \\
B_{n}=\frac{-\Omega_{n} \beta_{n}-\xi_{n} \omega_{n} \alpha_{n}}{\omega_{D n}}
\end{gathered}
$$

Para obtener los resultados dinámicos del análisis la solución 2.88 se ha implementado en el entorno $\mathrm{C}++$ por su eficiencia de cálculo.

Para los primeros 0.6 segundos se obtienen en centro de vano los resultados de desplazamientos representados en la figura 2.7 .

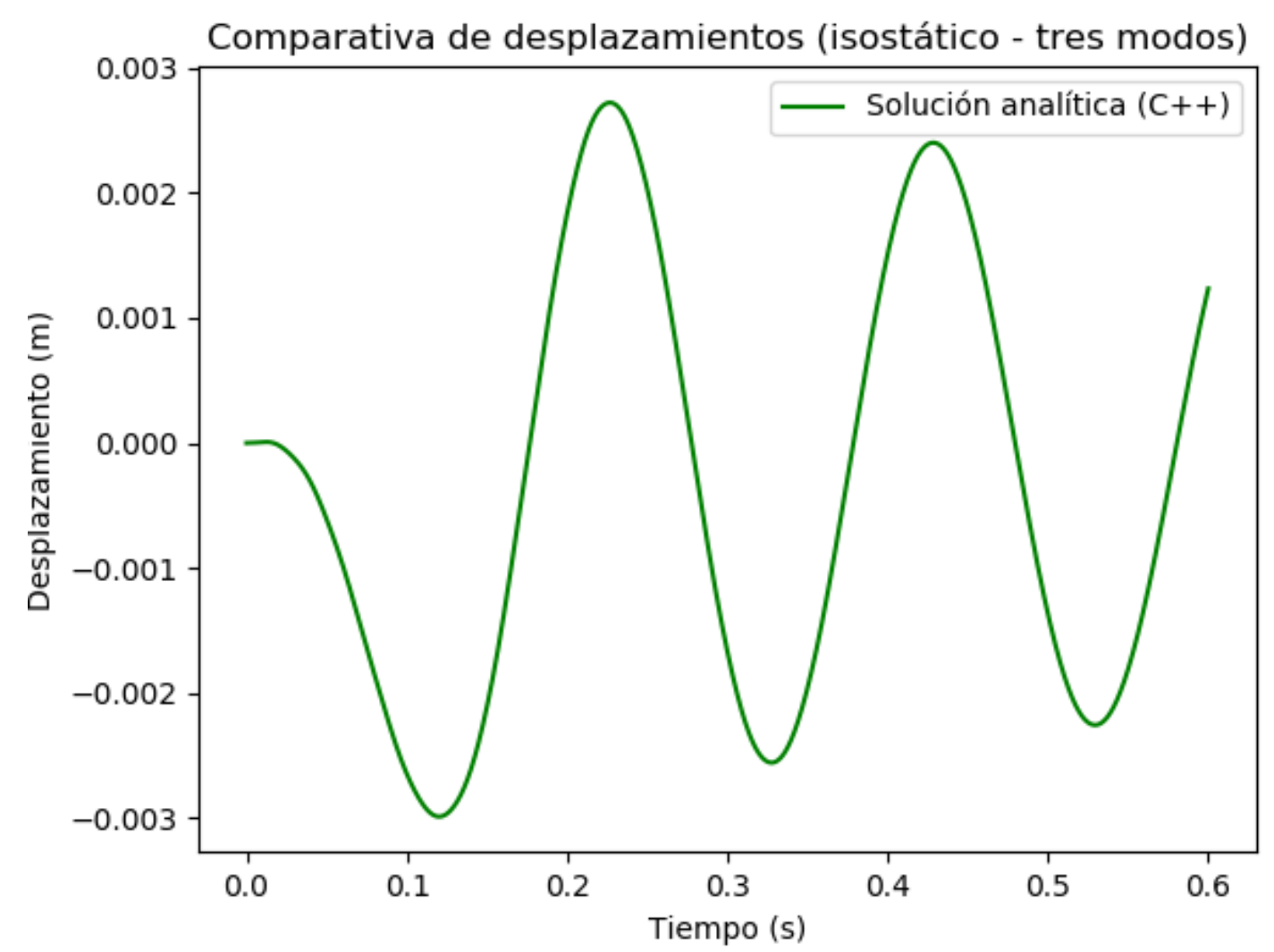

Figura 2.7: Desplazamientos en centro de vano de manera analítica. Prueba 1

Para el mismo período de tiempo y la misma ubicación los resultados de aceleración son los representados en la figura 2.8 . 


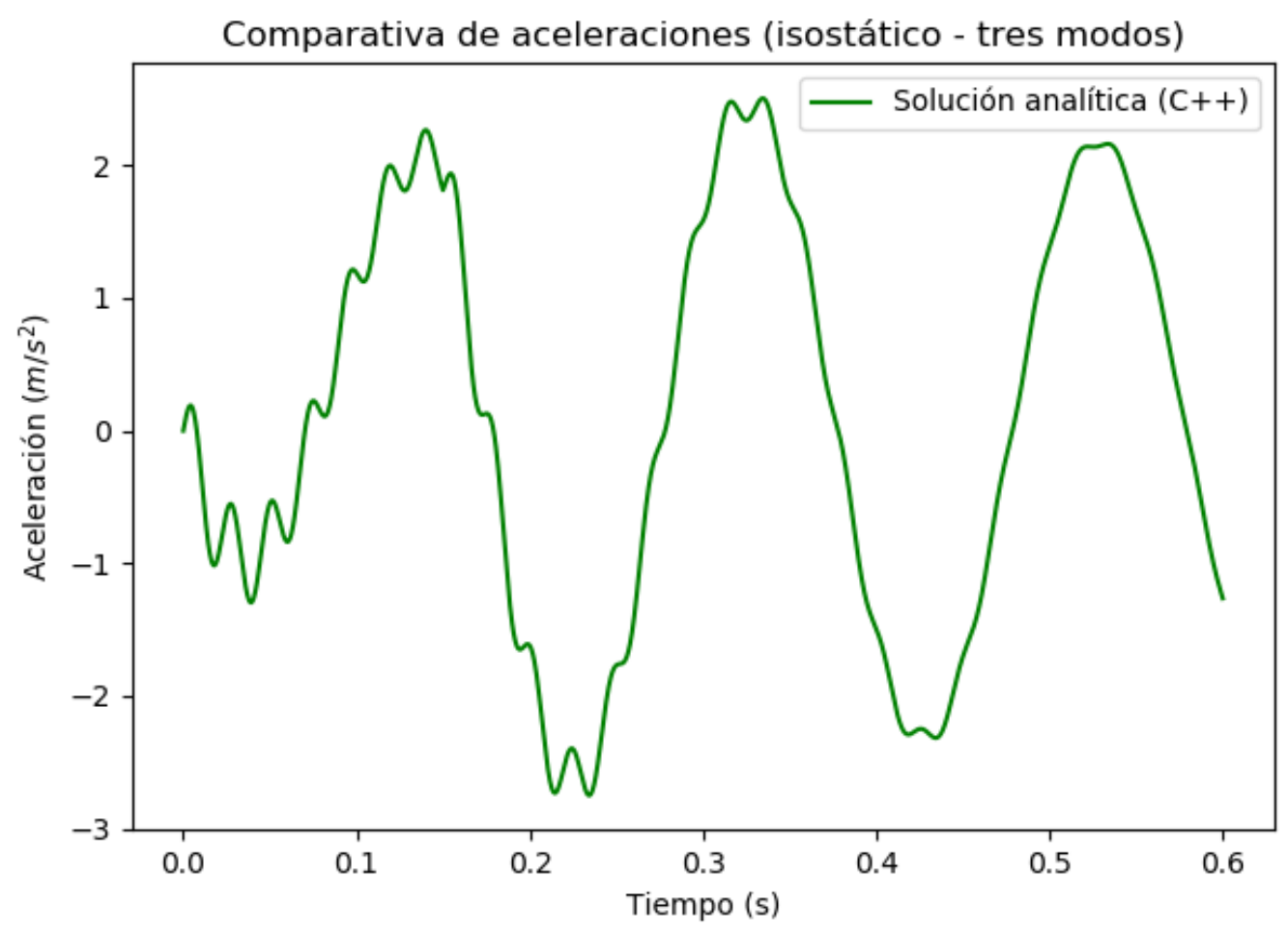

Figura 2.8: Aceleración de centro de vano de manera analítica. Prueba 1

\section{Cálculo mediante SAP2000}

Como en cualquier método paso a paso, se debe definir los dos siguientes parámetros:

1. Tiempo de discretización de la carga: Se emplearán pasos de $3 \cdot 10^{-4} \mathrm{~s}$.

2. Tiempo de discretización del paso: Será igual que el anterior.

Los pasos se eligen con lo suficientemente finos para poder comparar con los otros métodos pero teniendo el cuidado de no seleccionar un número de pasos demasiado elevado, ya que este método conlleva un coste computacional muy alto.

Para los primeros 0.6 segundos se obtienen en centro de vano los resultados de desplazamientos representados en la figura 2.9. 


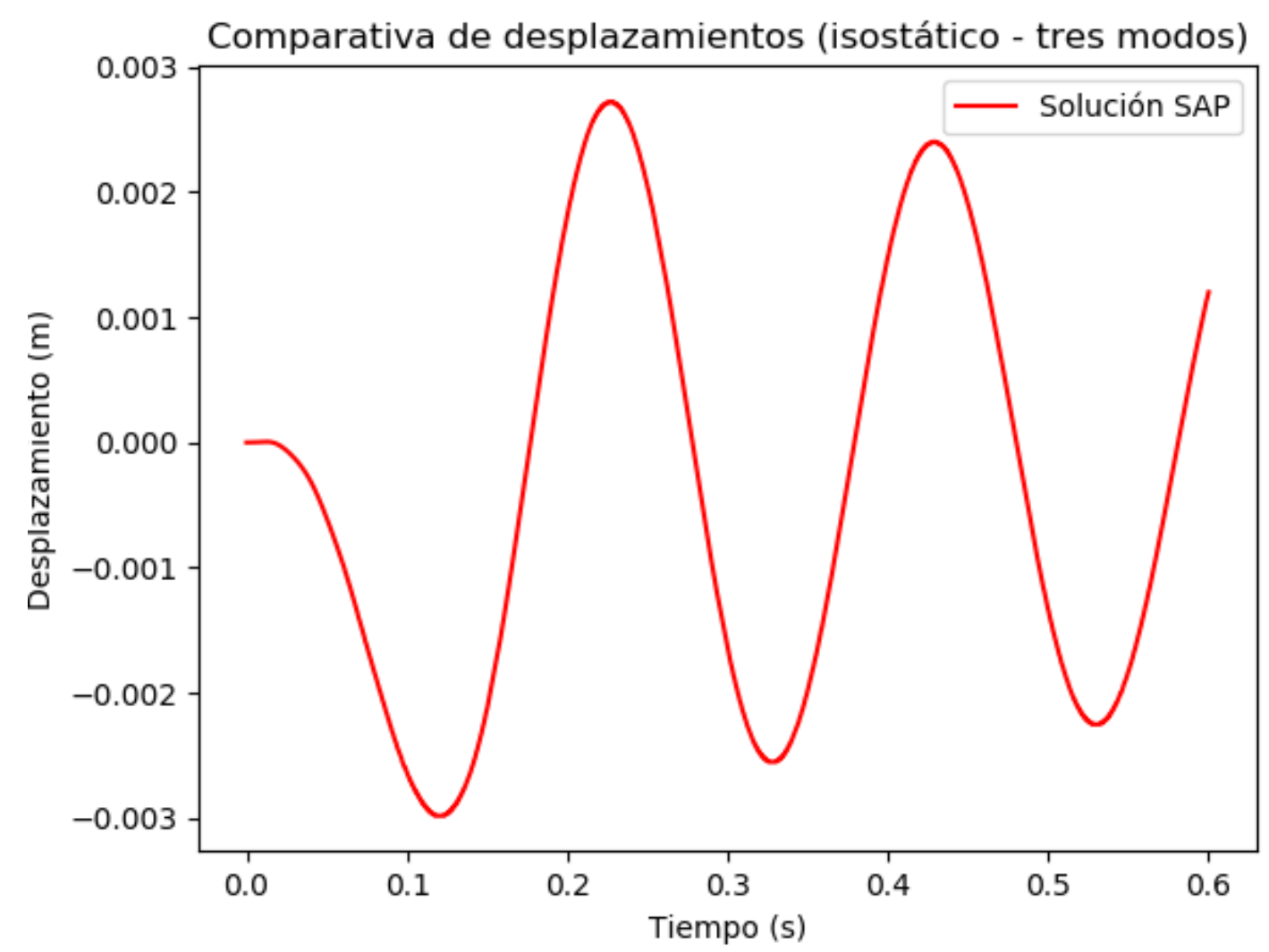

Figura 2.9: Desplazamientos en centro de vano mediante SAP2000. Prueba 1

Para el mismo período de de tiempo y la misma ubicación los resultados de aceleración son los representados en la figura 2.10 . 


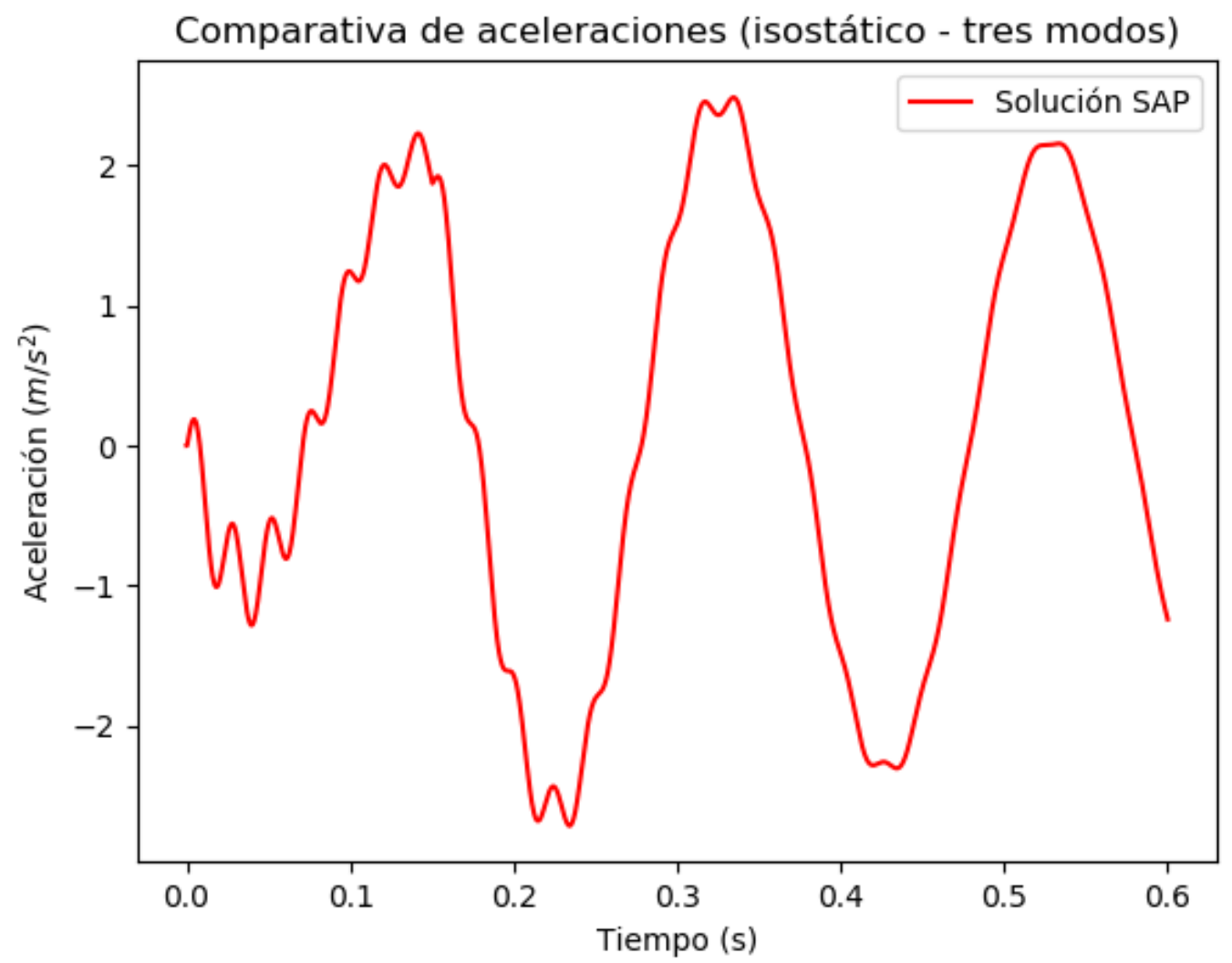

Figura 2.10: Aceleración en centro de vano mediante SAP2000. Prueba 1

\section{Cálculo método semianalítico}

Los datos de entrada del método semianalítico son: frecuencias naturales, número de modos a analizar, valores de aceleración vertical y giros procedentes del análisis modal, valor de la amortiguación, definición de cargas móviles, definición de línea de carga y de puntos de postproceso. El poder definir exclusivamente los puntos que interesa analizar ahorrará más tiempo ya que los cálculos en SAP2000 se realizan en cada nodo que se discretiza la estructura.

El paso de tiempo empleado es el mismo que en el cálculo con SAP2000 y se han empleado como puntos de posproceso cuartos y centro de vano.

Para los primeros 0.6 segundos se obtienen en centro de vano los resultados de desplazamientos representados en la figura 2.11. 


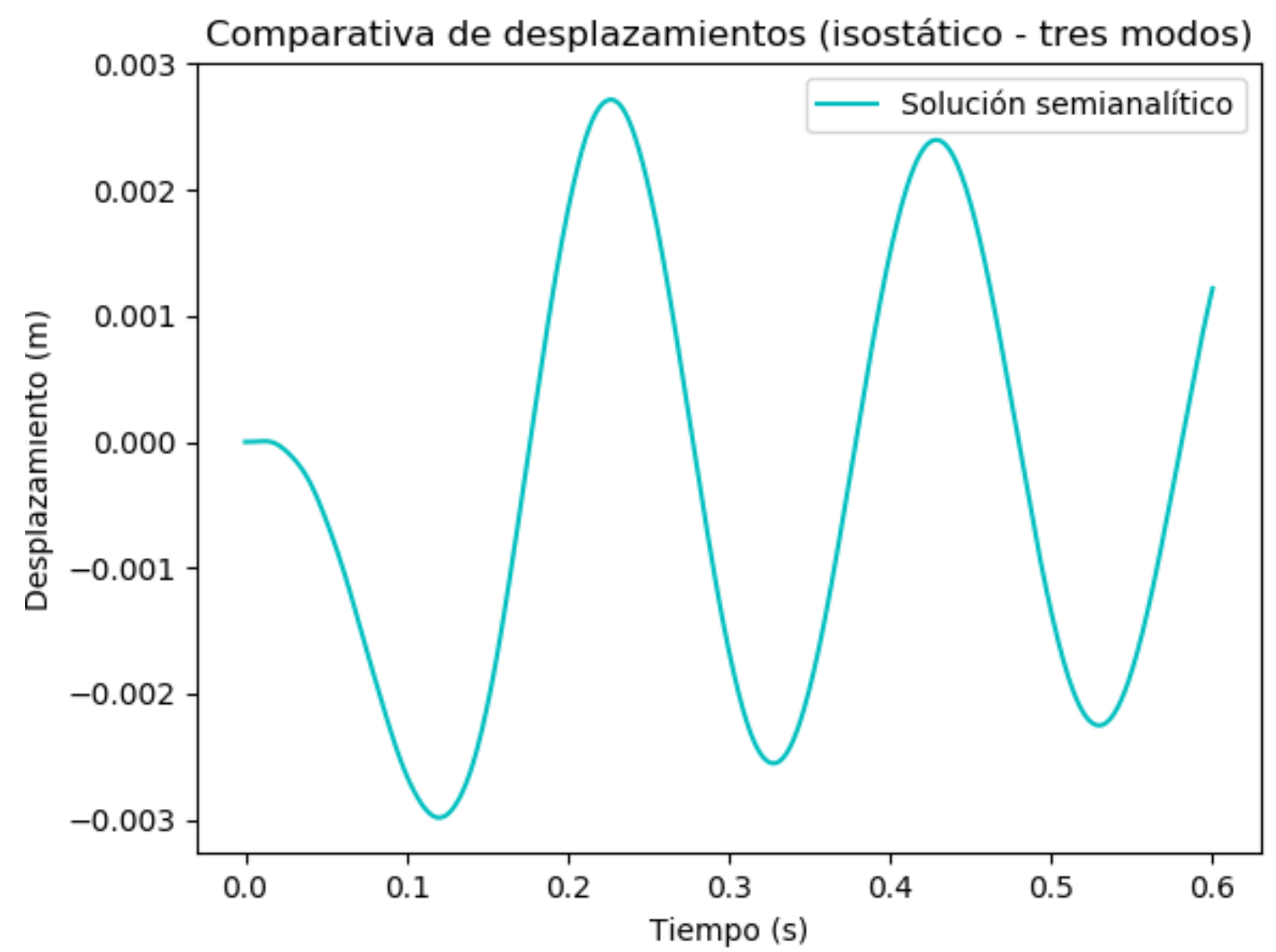

Figura 2.11: Desplazamientos en centro de vano mediante el método semianalítico. Prueba 1

Para el mismo período de de tiempo y la misma ubicación los resultados de aceleración son los representados en la figura 2.12. 


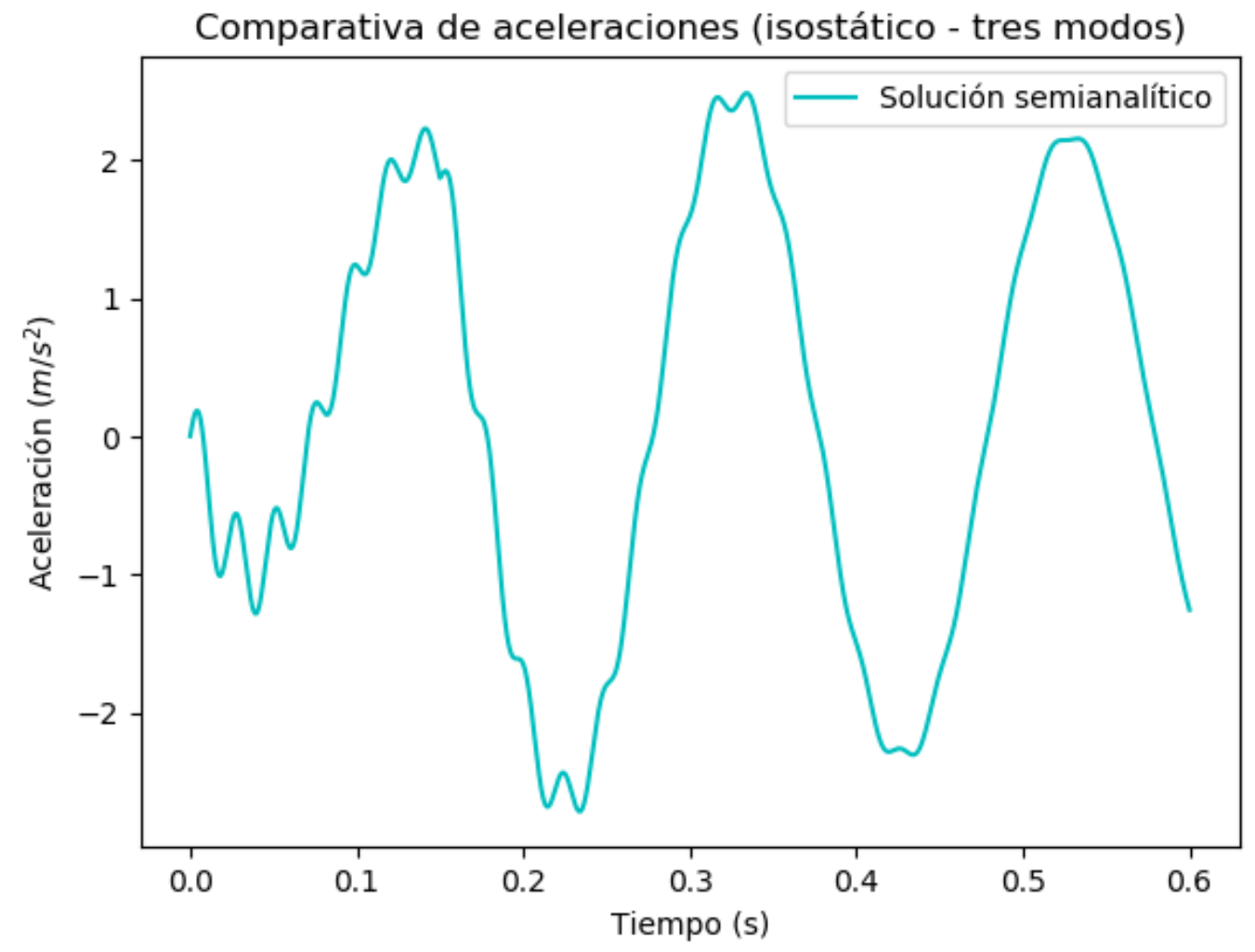

Figura 2.12: Aceleración en centro de vano mediante el método semianalítico. Prueba 1

\section{Comparativa}

La comparativa de resultados de desplazamiento se puede observar en la figura 2.13.

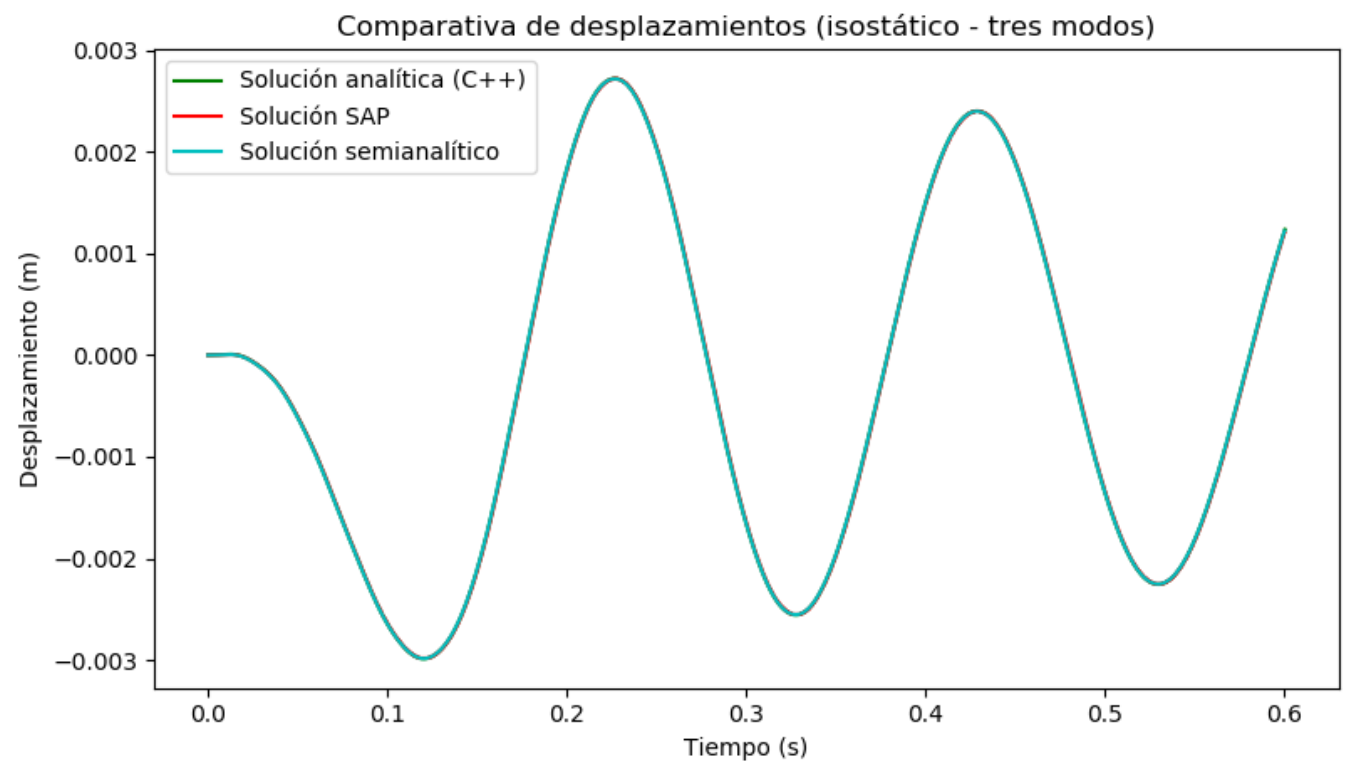

Figura 2.13: Comparativa de desplazamientos. Prueba 1 
La comparativa de resultados de desplazamiento se puede observar en la figura 2.14.

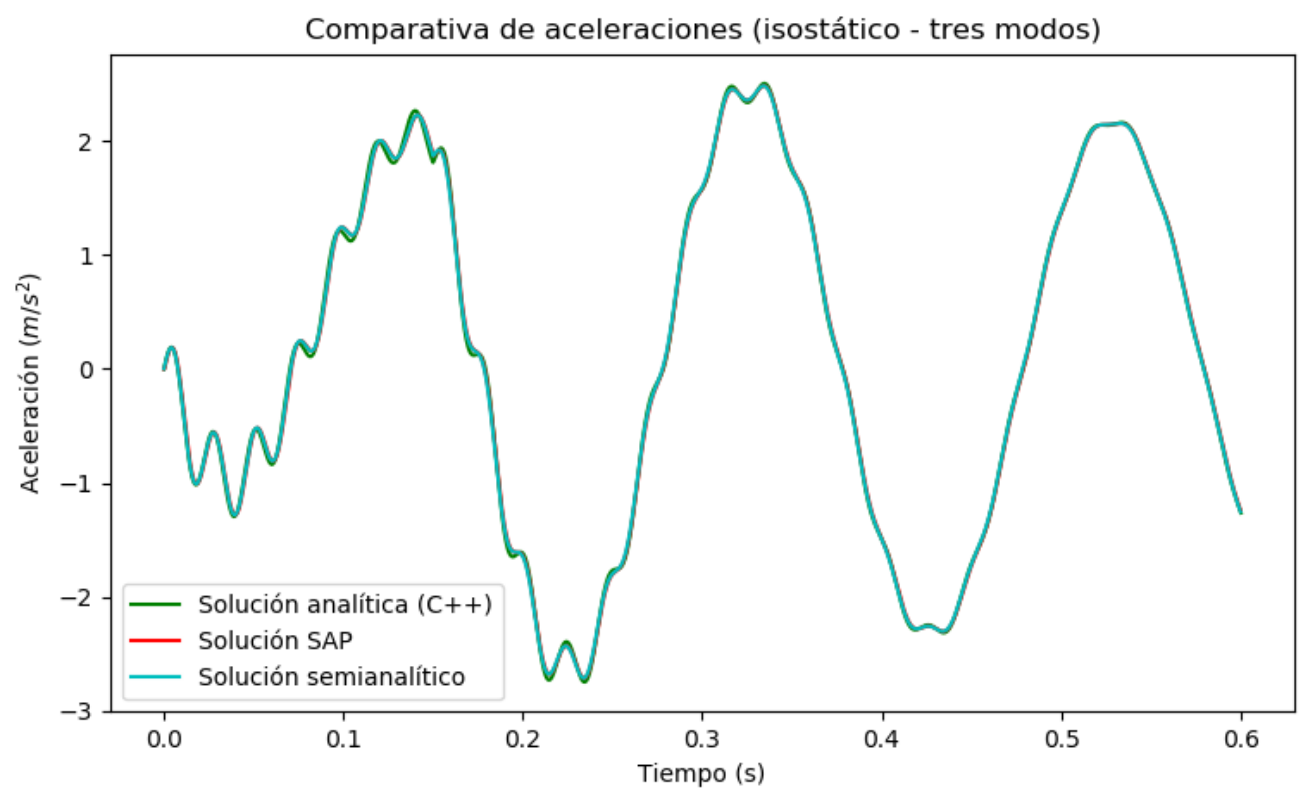

Figura 2.14: Comparativa de aceleración. Prueba 1

Como se puede observar en las figuras 2.13 y 2.14 los tres métodos arrojan el mismo resultado. También cabe destacar que el tiempo de computación de los métodos analítico y semianalítico es mucho menor que el empleado por SAP2000, lo que los convierte en métodos de cálculo mucho más recomendables.

\subsubsection{Prueba de validación 1: Viga de Bernouilli-Euler de tres vanos}

Esta segunda prueba consiste en la comparativa de resultados dinámicos tras el paso de una carga móvil por la viga representada en la figura 2.15 .

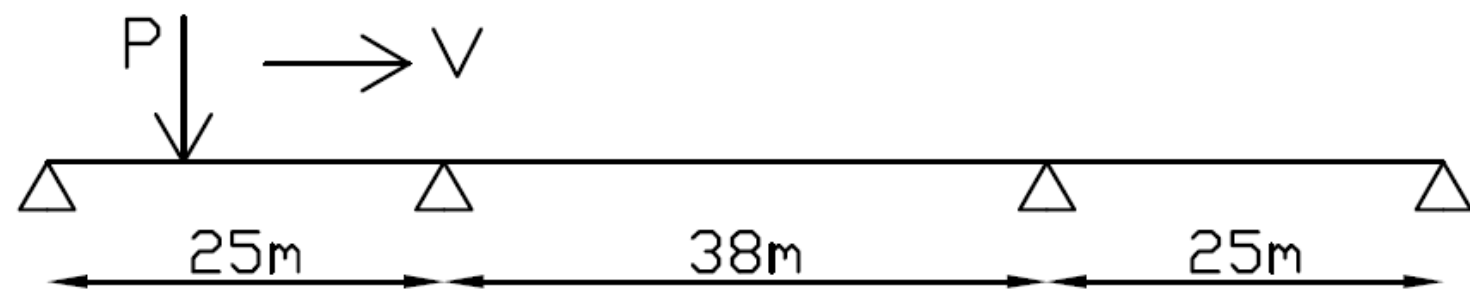

Figura 2.15: Geometría de la viga. Prueba 2

Las características de la viga son:

- Viga de Bernouilli - Euler

- Masa por unidad de longitud: $\rho \mathrm{A}=14.435 \mathrm{t} / \mathrm{m}^{3}$ 
- Rigidez a flexión: $\mathrm{EI}=110.6 \mathrm{GNm}^{2}$

- Amortiguamiento modal: $\xi=2 \%$

- Velocidad de paso: $\mathrm{V}=100 \mathrm{~m} / \mathrm{s}$

- Carga móvil: $\mathrm{P}=195 \mathrm{kN}$

Se divide la viga de manera uniforme en 8 elementos. Se realiza un análisis bidimensional y el cálculo modal mediante el software SAP2000 considerando 5 modos de vibración.

Los resultados de períodos y frecuencias naturales obtenidos en el análisis modal son los representados en la tabla 2.2 .

\begin{tabular}{|c|c|c|}
\hline Modo $\left(\mathrm{n}^{\mathrm{o}}\right)$ & Frecuencia $(\mathrm{Hz})$ & Período $(\mathrm{s})$ \\
\hline 1 & 4.2802 & 0.2336 \\
\hline 2 & 8.1962 & 0.1220 \\
\hline 3 & 9.5928 & 0.1042 \\
\hline 4 & 15.8050 & 0.0633 \\
\hline 5 & 27.4730 & 0.0364 \\
\hline
\end{tabular}

Cuadro 2.2: Frecuencias y períodos de vibración. Prueba 2

Una vez realizado el análisis modal se va a proceder al análisis dinámico de la viga mediante el paso de una carga puntual. El análisis dinámico se va a efectuar a través de los siguientes métodos:

1. Programa comercial SAP2000

2. Método semianalítico

\section{Cálculo mediante SAP2000}

Los pasos de tiempo elegidos son los iguales a los utilizados en el caso de la viga de tipología biapoyada.

1. Tiempo de discretización de la carga: Se emplearán pasos de $3 \cdot 10^{-4} \mathrm{~S}$.

2. Tiempo de discretización del paso: Será igual que el anterior.

Para los primeros 0.6 segundos se obtienen en el centro del vano central los resultados de desplazamientos representados en la figura 2.16 


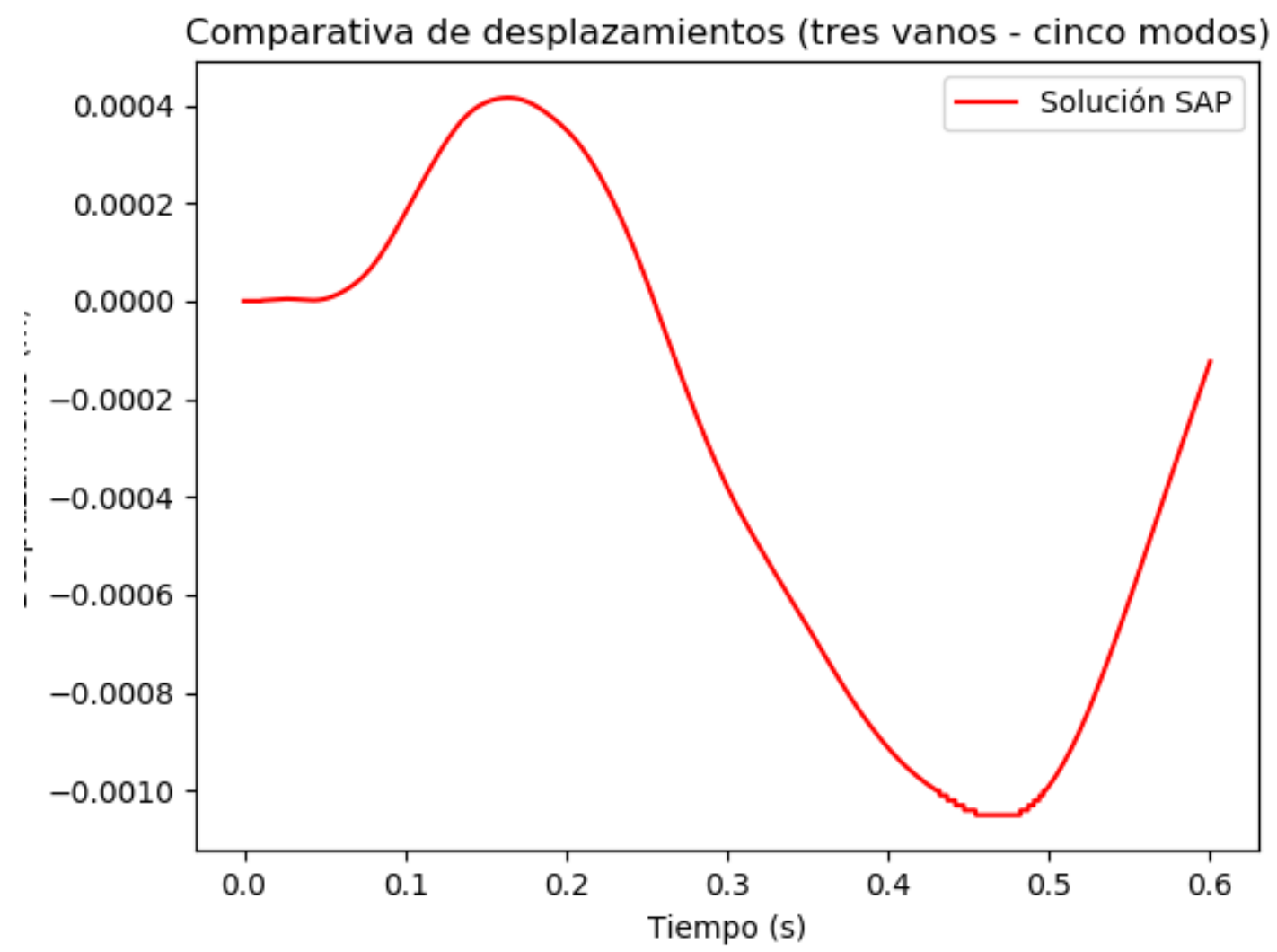

Figura 2.16: Desplazamientos en centro de vano central de SAP2000. Prueba 2

Para el mismo período de de tiempo y la misma ubicación los resultados de aceleración son los representados en la figura 2.17. 


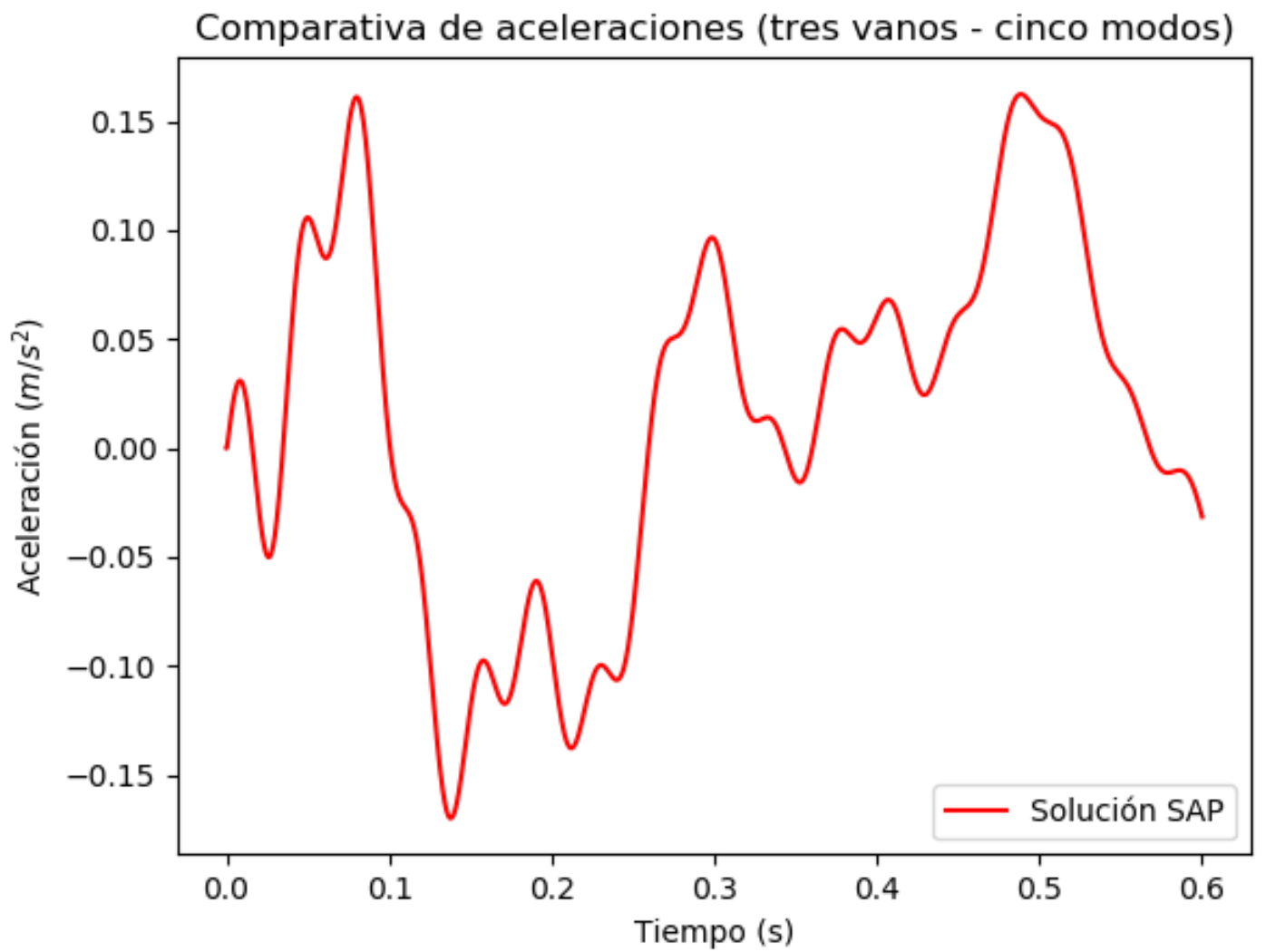

Figura 2.17: Aceleración en centro de vano central de SAP2000. Prueba 2

\section{Cálculo método semianalítico}

Los puntos de posproceso elegidos son los situados en cuartos y centro de vano.

Para los primeros 0.6 segundos se obtienen en el centro del vano central los resultados de desplazamientos representados en la figura 2.18 


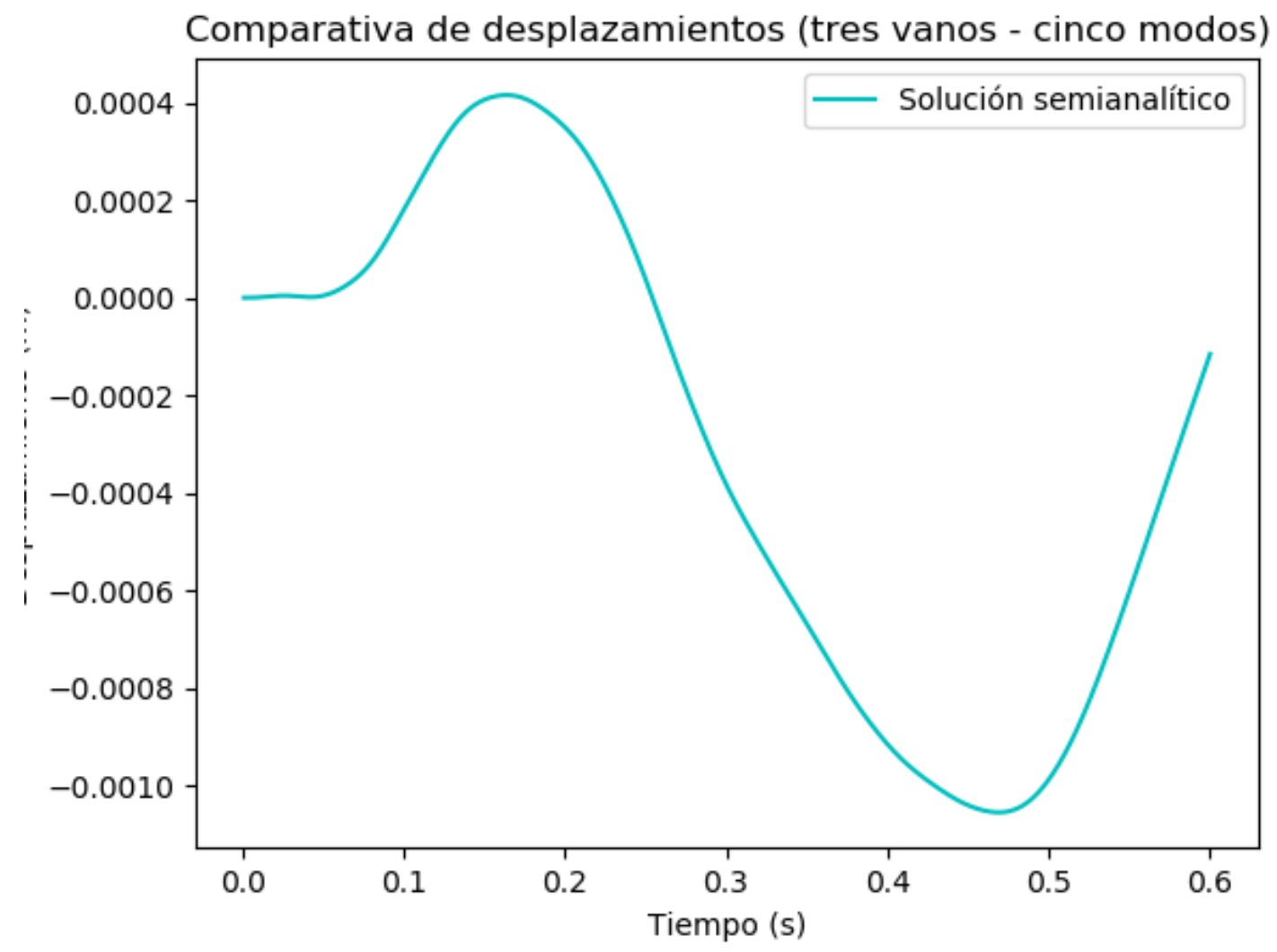

Figura 2.18: Desplazamientos en centro de vano central del método semianalítico. Prueba 2

Para el mismo período de de tiempo y la misma ubicación los resultados de aceleración son los representados en la figura 2.19. 


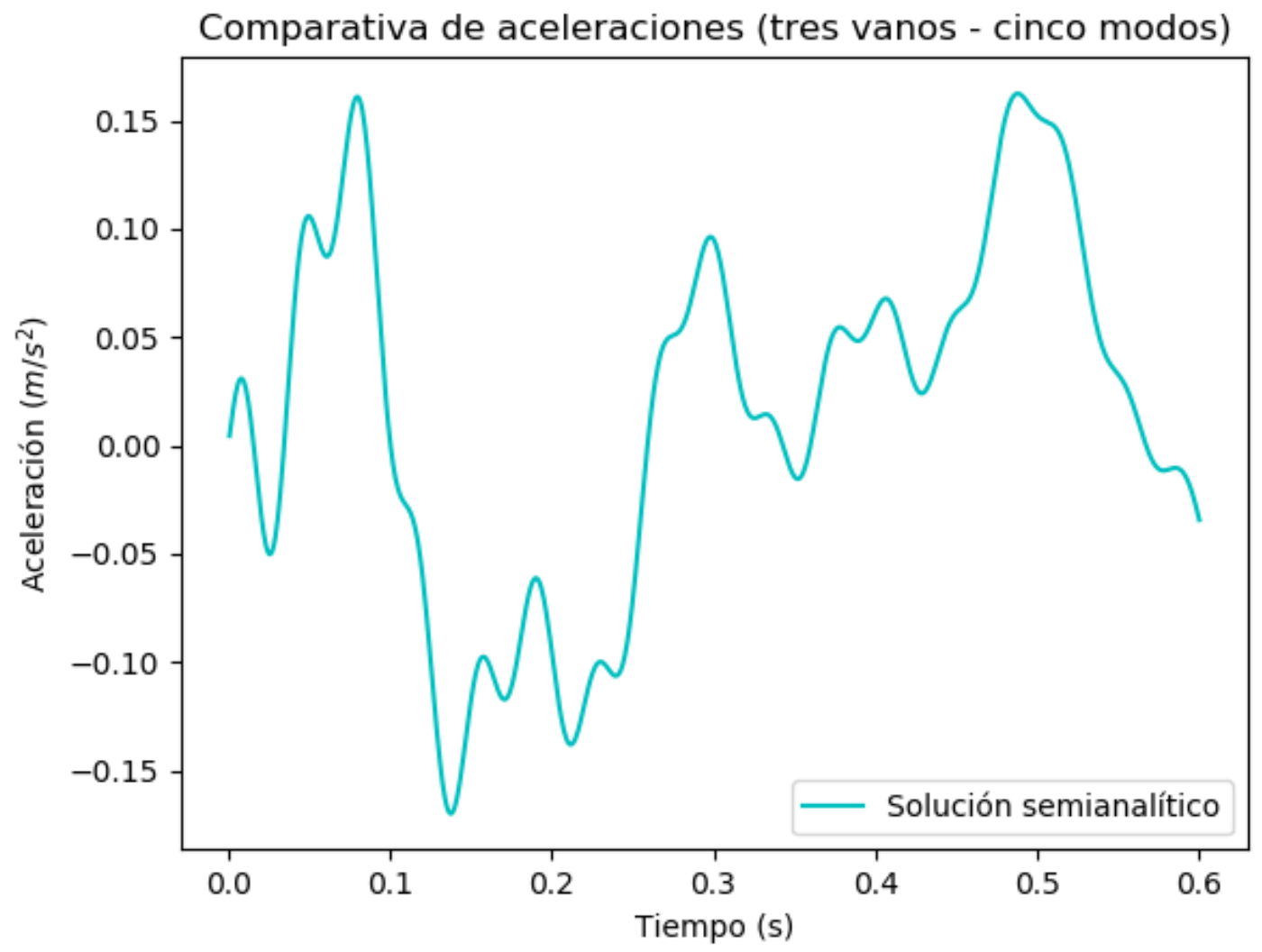

Figura 2.19: Aceleración en centro de vano central del método semianalítico. Prueba 2

\section{Comparativa}

La comparativa de los resultados de desplazamiento se puede observar en la figura 2.20. 


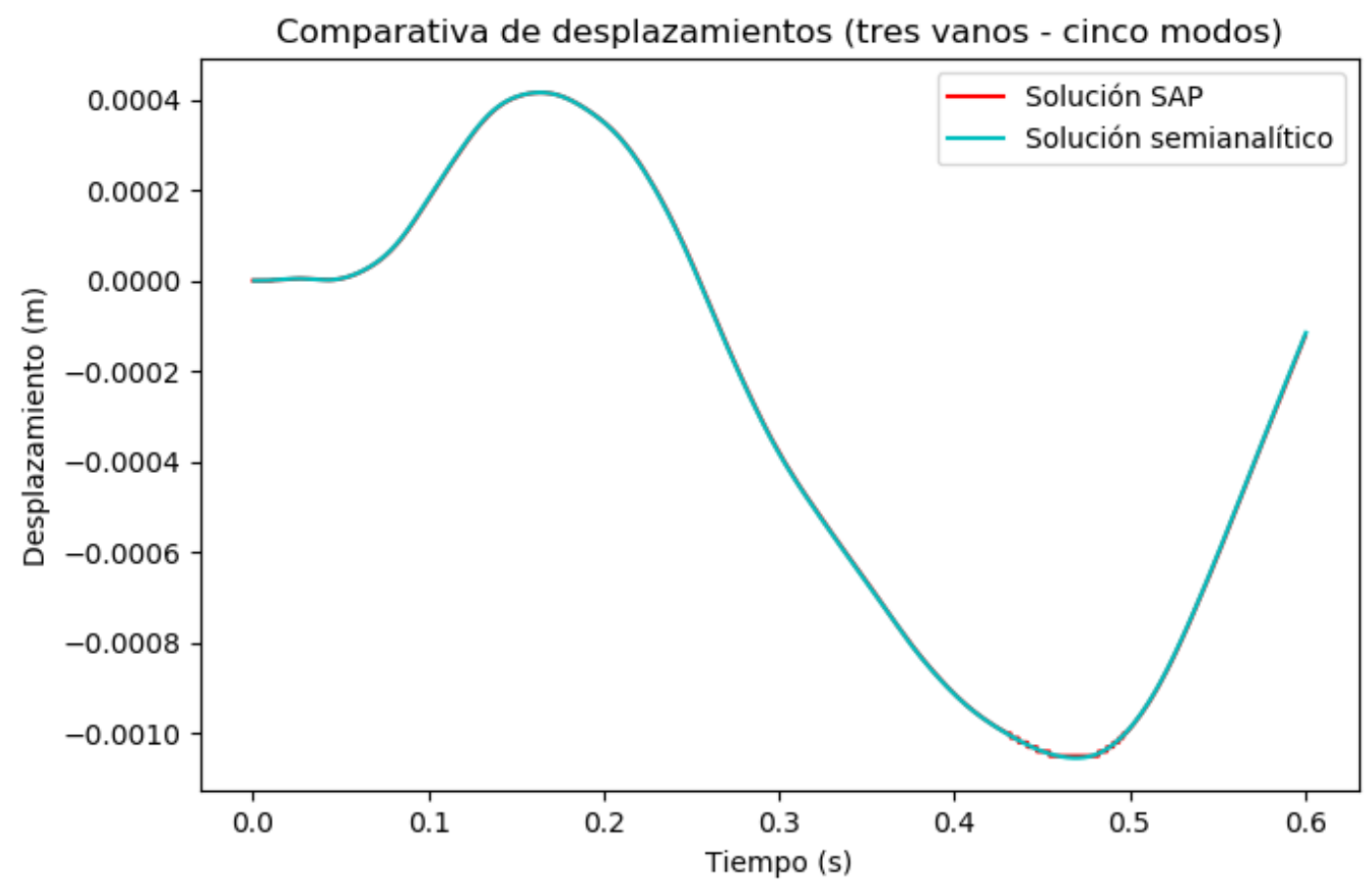

Figura 2.20: Comparativa de desplazamientos en el centro del vano central

La comparativa de los resultados de aceleración se puede observar en la figura 2.21. 


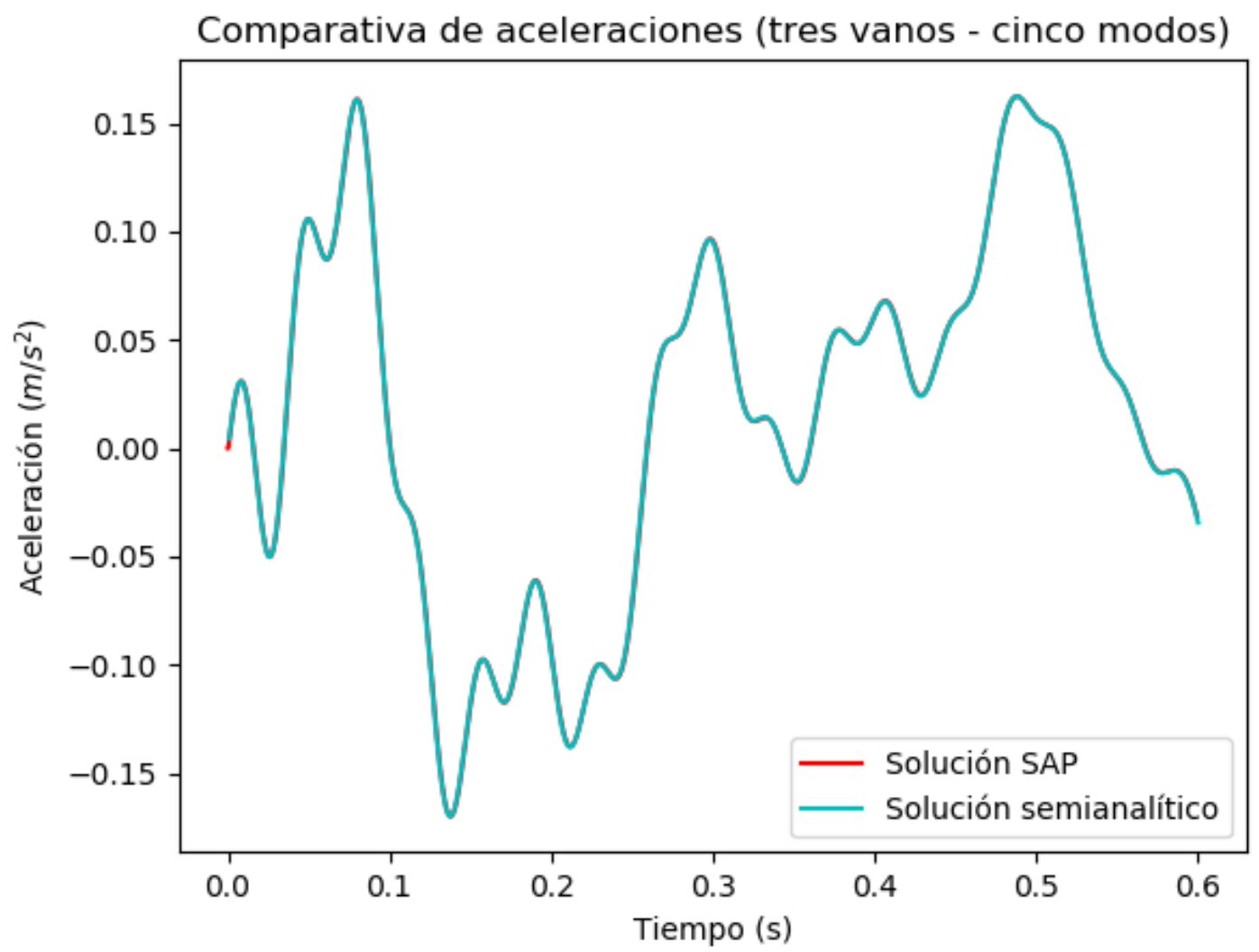

Figura 2.21: Comparativa de aceleración en el centro del vano central

Como se puede observar en las figuras 2.20 y 2.21 los diferentes métodos vuelven a coincidir de forma exacta en los resultados. En lo que se refiere al tiempo de computación de nuevo se ha obtenido un tiempo muy superior en SAP2000, lo que hace que el método semianalítico sea mucha más eficiente y recomendable para el análisis de series temporales.

\subsection{Conclusiones}

A continuación se hace una exposición de las conclusiones a las que se ha llegado tras la realización de las pruebas de validación.

1. La utilización del método semianalítico implica una disminución del tiempo empleado en el cálculo. Este ahorro de tiempo procede de la formulación analítica de la variable temporal, evitando el empleo de métodos de integración en el dominio del tiempo. Los métodos paso a paso ven condicionada su fiabilidad y los tiempos de cálculo al intervalo de paso seleccionado, el cual debe tomar un valor muy pequeño. Por otro lado, el método semianalítico ofrece la posibilidad de escoger los puntos de posproceso en los que se obtienen resultados, lo que elimina gran número de cálculos ya que no se analizan todos los puntos discretizados. 
2. Los métodos paso a paso pierden fiabilidad a medida que el intervalo elegido aumenta su valor, lo que produce una acumulación de errores en cada iteración. En el cálculo realizado mediante el método semianalítico no existe esta problemática ya que se realiza un cálculo exacto en la variable temporal. Los errores acumulados en el empleo del método semianalítico provienen exclusivamente de los cometidos en el análisis modal realizado a partir del método de elementos finitos.

3. Los intervalos de tiempo de evaluación utilizados en el empleo del método semianalítico son menores a los intervalos de tiempo de integración necesitados por los métodos de integración paso a paso. Un intervalo igual a una décima parte del período más pequeño asegura resultados satisfactorios, mientras que en los métodos paso a paso la definición de esta variable condiciona la rigurosidad del cálculo.

4. El método semianalítico proporciona la solución utilizando un total de diez coeficientes por elemento y modos que no son dependientes de la velocidad. Este proceso convierte al método en el óptimo para realizar los numerosos cálculos exigidos por la normativa vigente en materia ferroviaria.

5. A bajas velocidades la solución obtenida mediante el método semianalítico converge a la estática, por lo que se evita el fenómeno de Gibbs asociado a las soluciones analíticas basadas en series armónicas, las cuales deben sufrir un tratamiento especial.

6. Durante el empleo del método semianalítico se evita el uso de funciones exponenciales con argumentos elevados, que conllevan errores numéricos en soluciones analíticas. Esto es debido a la normalización temporal realizada en la formulación de la metodología. 


\section{CAPÍtulo 3}

\section{Interacción suelo-estructura. Análisis modal}

\subsection{Trabajos y publicaciones}

La interacción suelo-estructura es un tema sobre el que se han publicado diversos trabajos en los últimos años. Se va a realizar una síntesis de los mismos con el fin de comprender el tema sobre el que versa el presente trabajo.

Ülker Kaustell et al. [12] llevaron a cabo un estudio sobre el caso de la interacción sueloestructura en un puente de vigas semejante a un pórtico perteneciente a la línea de Botnia, Suecia. Para el estudio se consideraron las siguientes tres hipótesis de estructura:

1. Empotrada

2. Con interacción suelo-estructura estática

3. Con interacción suelo estructura dinámica

En la imagen 3.1 se pueden observar las condiciones en los apoyos de cada una de las hipótesis asumidas. 

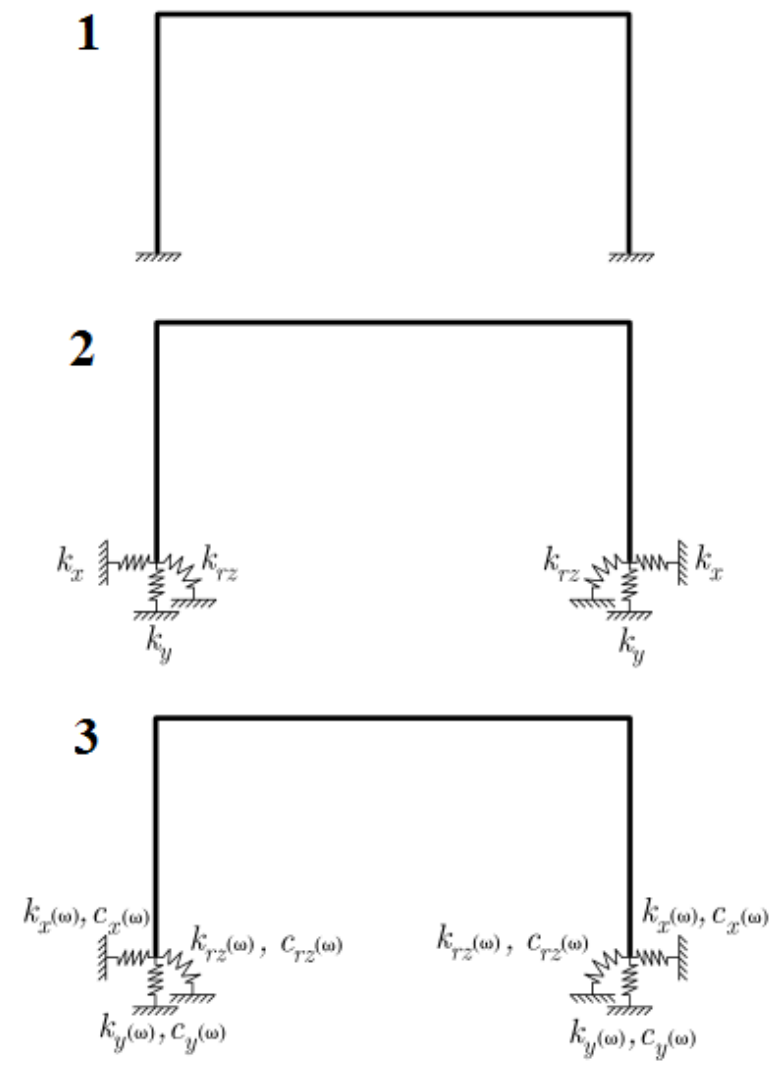

Figura 3.1: Alternativas estudiadas. Ülker Kaustell et al. [12]

Una crítica que se debe realizar al modelo dinámico es la no inclusión de la masa de la cimentación ya que es una magnitud fundamental en el estudio de estructuras bajo el paso de cargas móviles.

En la figura 3.2 se observan los resultados de aceleración máximos obtenidos para el paso de la carga. 

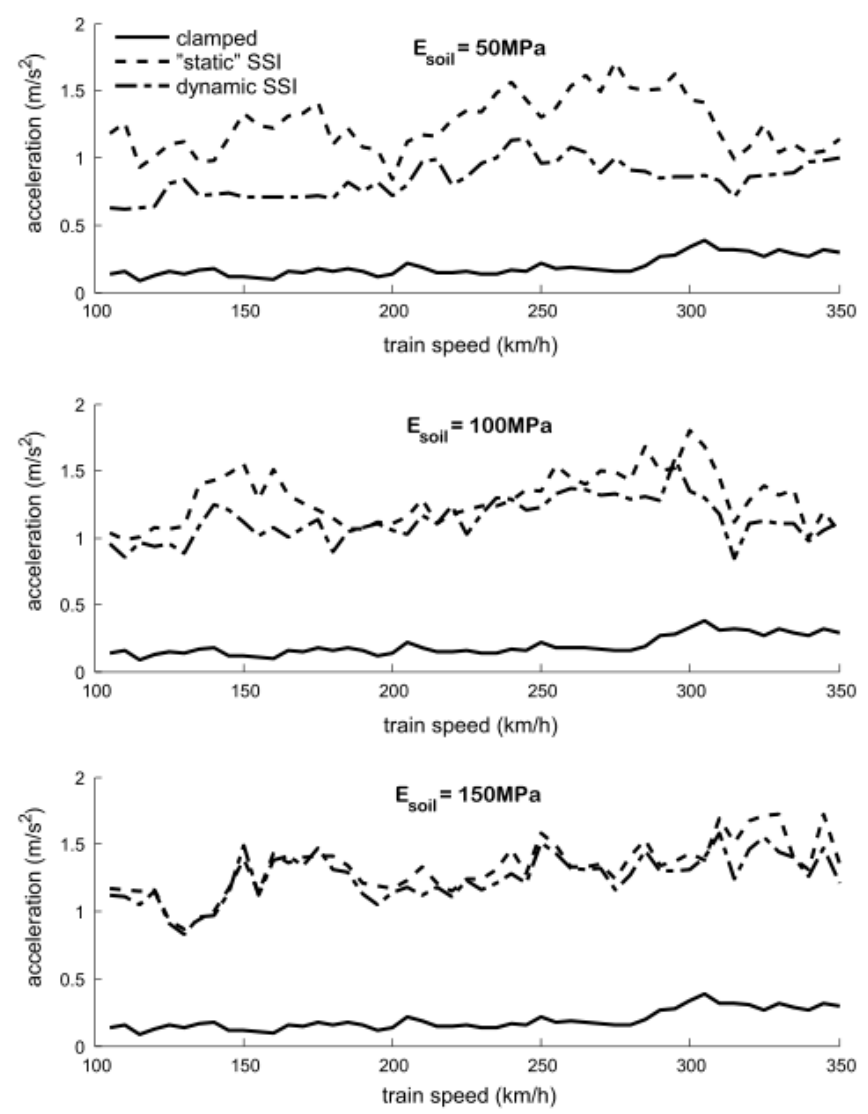

Figura 3.2: Valores máximos de aceleración. Ülker Kaustell et al. [12]

El resultado de la estructura empotrada está muy lejos de reflejar la realidad de la situación. En cuanto a los dos modelos con interacción se observa como el modelo estático devuelve unos valores de mayor magnitud, algo evidente debido a la no inclusión de amortiguadores. A medida que el terreno se vuelve más rígido las soluciones convergen, lo que indica que la tasa de amortiguamiento geométrico producido es menor a medida que el suelo posee una mayor rigidez.

Romero et al. [33] comenzaron a estudiar los efectos de la resonancia, llevando a cabo un estudio parecido al del Ülker Kaustell et al. [12], pero utilizando un modelo de elementos finitos y de contorno en tres dimensiones, mucho más complejo que el anterior(3.3). 


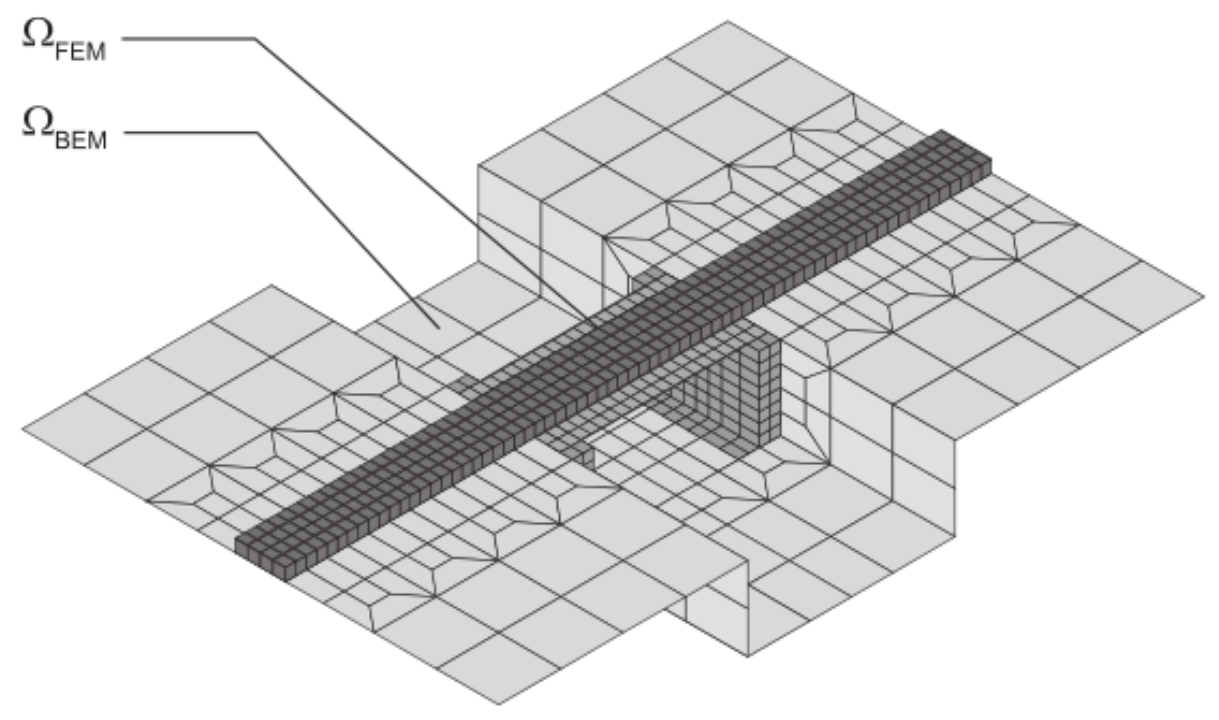

Figura 3.3: Discretización de la estructura. Romero et al. [33]

Para la transmisión de cargas también se usó un modelo mucho más complejo que incluía la representación del bogie del tren.

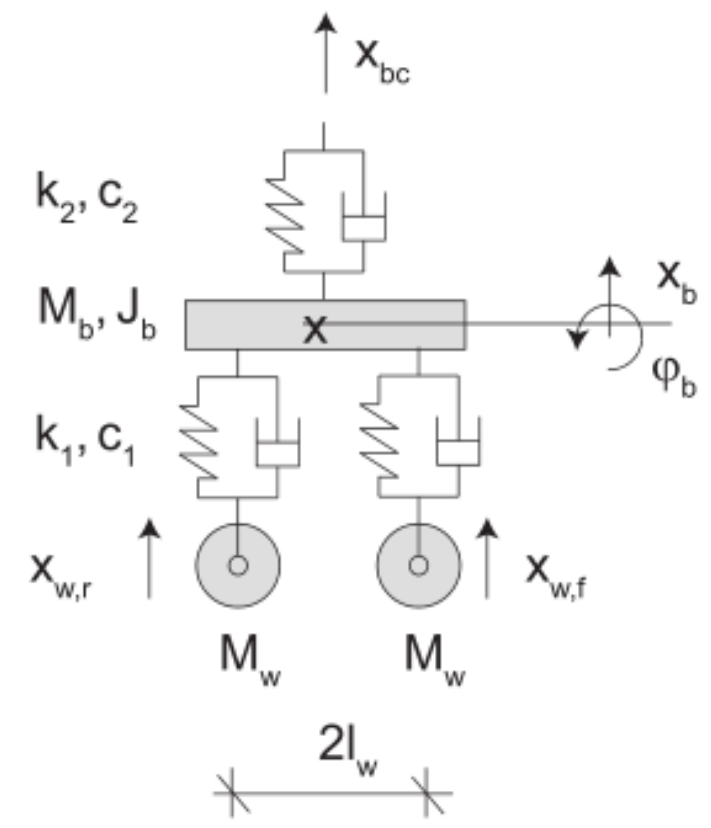

Figura 3.4: Modelo del bogie. Romero et al. [33]

Los resultados de aceleración obtenidos en centro de vano se pueden observar en la figura 3.5 . 

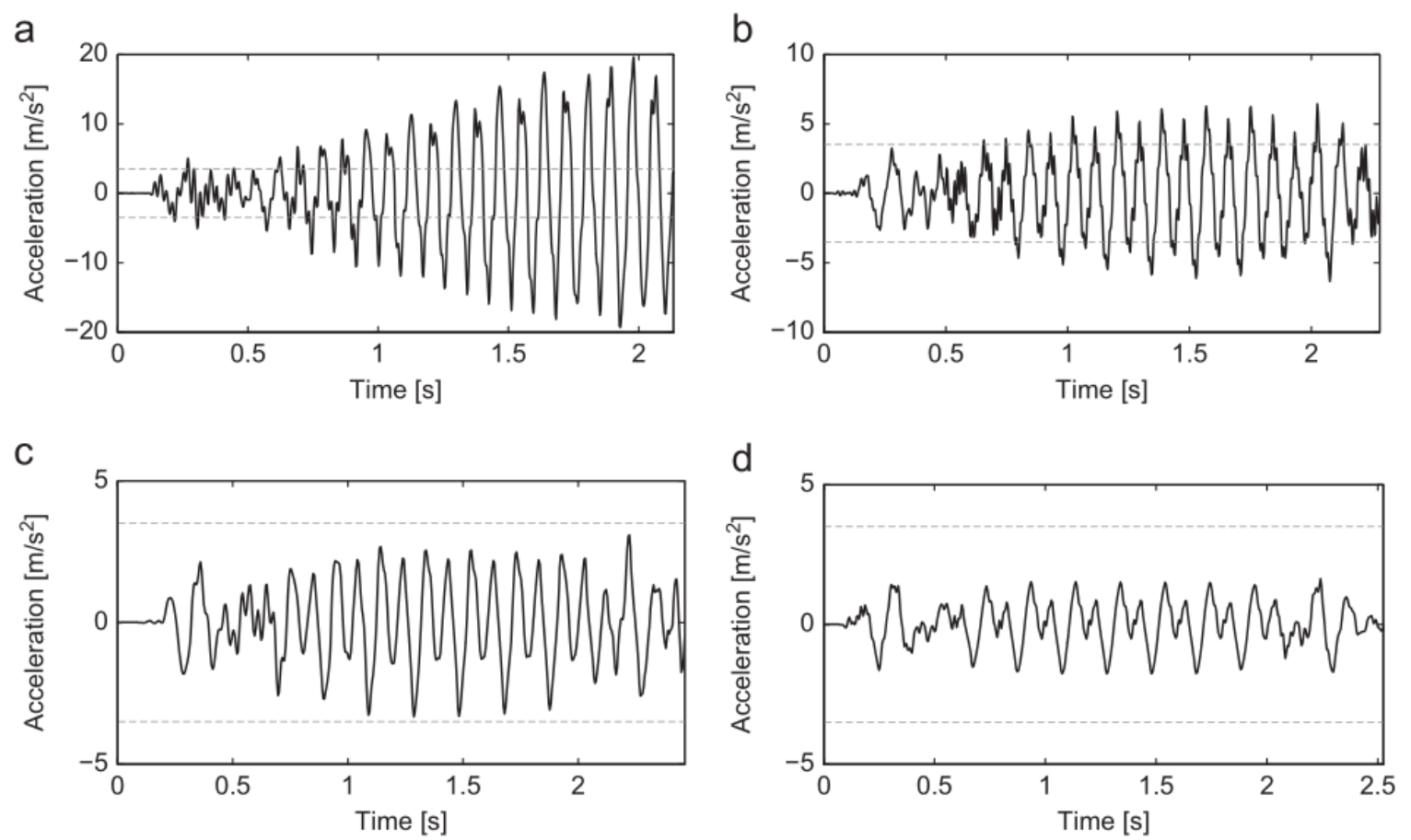

Figura 3.5: Serie temporal de aceleración en centro de vano para un tren viajando a (a) $\mathrm{v}=$ $110.4 \mathrm{~m} / \mathrm{s},(\mathrm{b}) \mathrm{v}=103 \mathrm{~m} / \mathrm{s},(\mathrm{c}) \mathrm{v}=95 \mathrm{~m} / \mathrm{s}$ y (d) $\mathrm{v}=92.5 \mathrm{~m} / \mathrm{s}$ en (a) terreno infinitamente rígido, (b) terreno duro, (c) terreno de dureza media y (d) terreno blando. Romero et al. [33]

Doménech et al. [4] y Martínez-Rodrigo et al. [15] también estudiaron los efectos provocados por la resonancia en elementos con interacción suelo-estructura. Para ello utilizaron el modelo representado en la figura 3.6, elaborado a partir de elementos finitos y de contorno y colocado sobre un semiespacio que representa el terreno. El modelo es muy complejo pero tiene un fallo y es la no inclusión de la masa de la cimentación. 


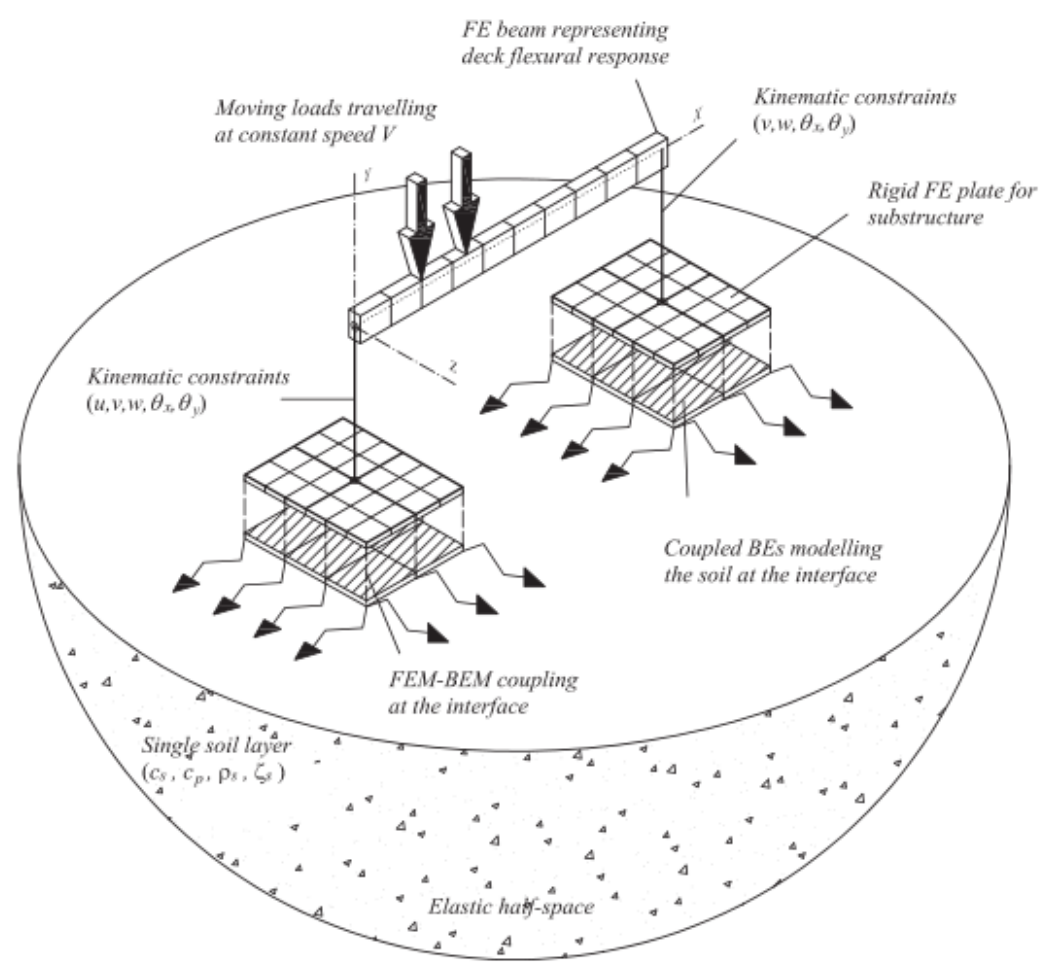

Figura 3.6: Modelo de elementos finitos y de contorno. Doménech et al. [4] y MartínezRodrigo et al. [15]

En la figura 3.7 se pueden observar los resultados de aceleración obtenidos en el trabajo de Doménech et al. [4]. Los resultados están compuestos por una envolvente de aceleraciones máximas y por una serie temporal a una velocidad de $222.58 \mathrm{~km} / \mathrm{h}$. Los resultados están referidos a centro de vano. 

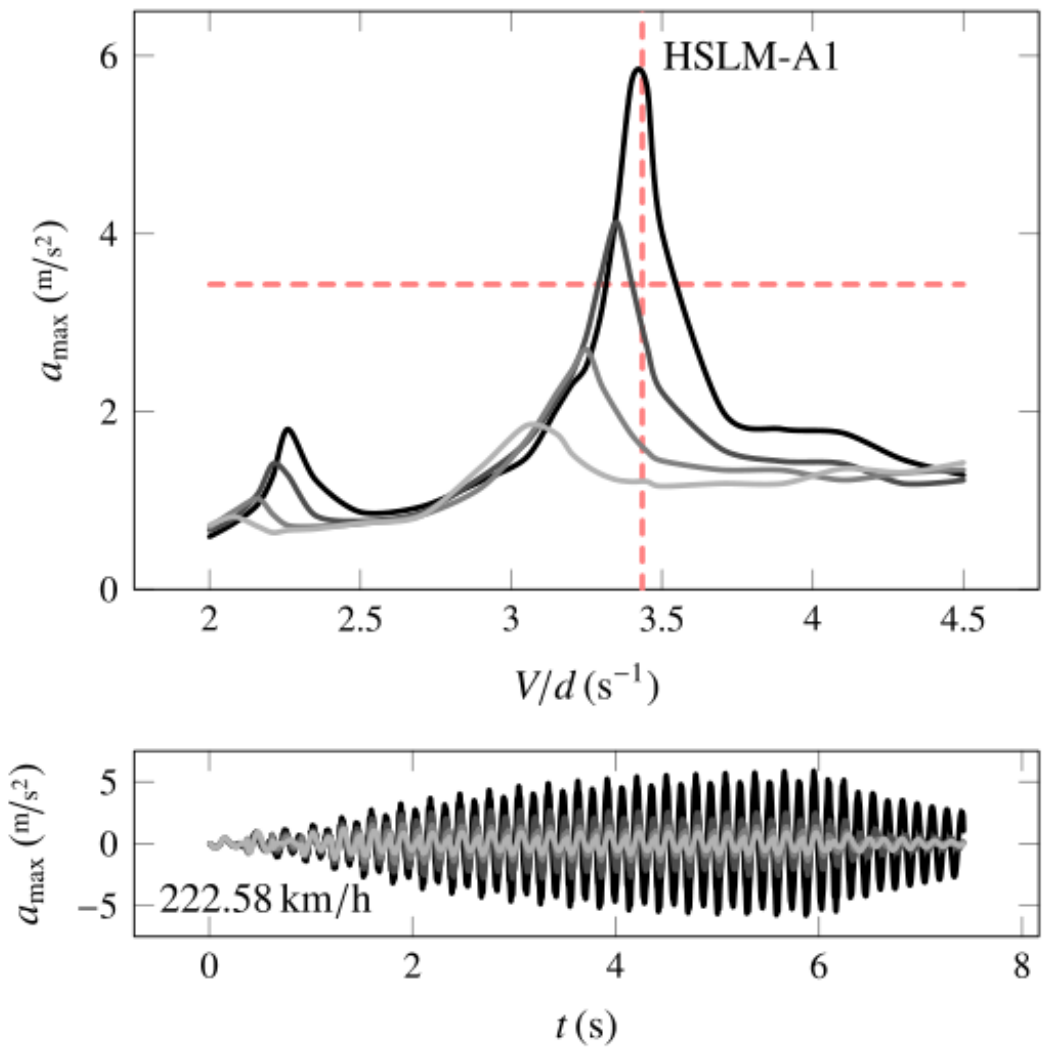

Figura 3.7: Resultados de aceleración. Doménech et al. [4]

En la figura 3.8 se aprecian los resultados de aceleración obtenidos por Martínez-Rodrigo et al. [15]. Se establece un amortiguamiento estructural constante igual al $5 \%$ y se realiza el análisis para cuatro tipos diferentes de terreno, desde suelo infinitamente rígido a blando. 


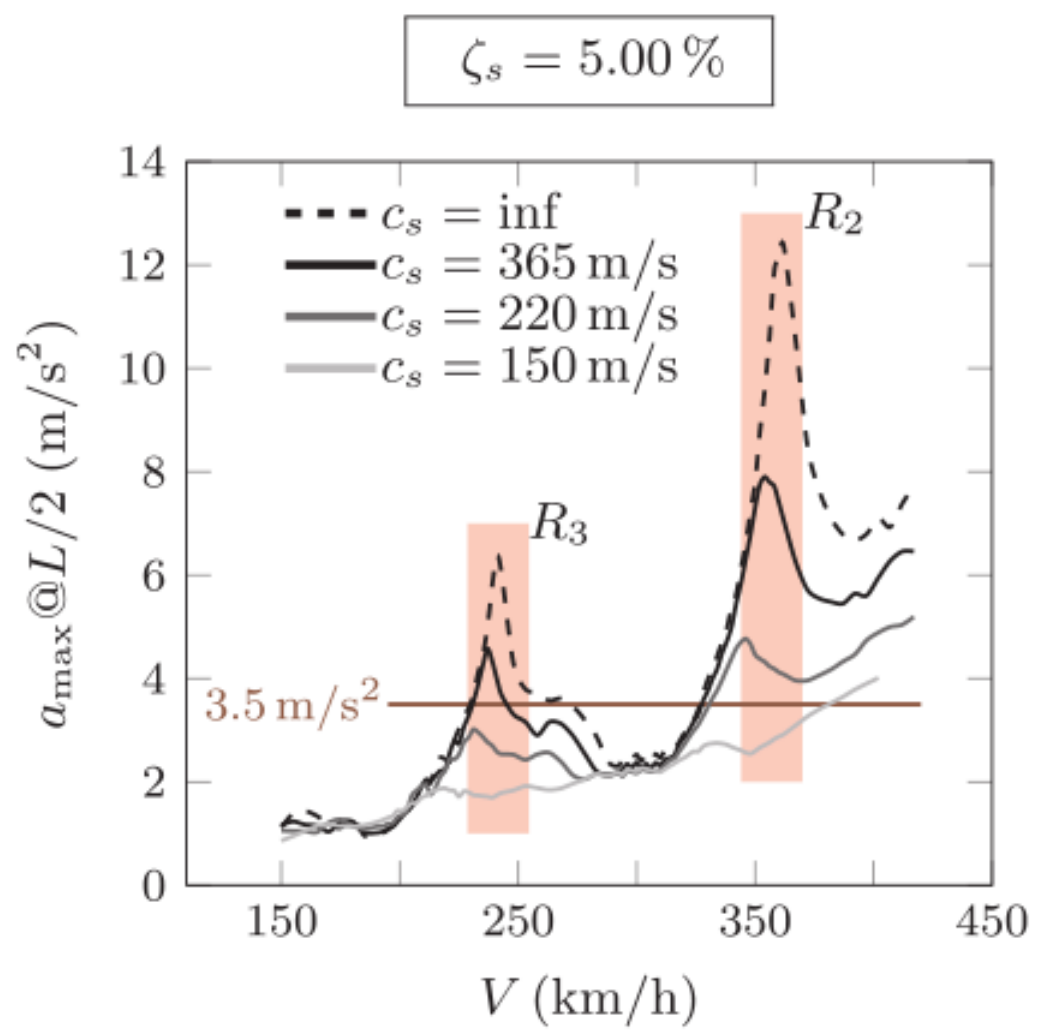

Figura 3.8: Resultados de aceleración. Martínez-Rodrigo et al. [15]

De los trabajos que han estudiado la influencia de la interacción suelo-estructura sobre los fenómenos de resonancia se extrae una conclusión muy clara, y es que cuanto menos rígido es el terreno sobre el que se sustenta la construcción menor es el valor de dichos efectos.

Para el cálculo de los resultados dinámicos obtenidos con anterioridad es necesario el establecimiento de los valores de rigidez y amortiguamiento en la dirección vertical. Recientemente Zangeneh et al. [40] publicaron un trabajo sobre la obtención de estos valores.

El terreno fue representado mediante el semiespacio que se puede observar en la figura 3.9. 


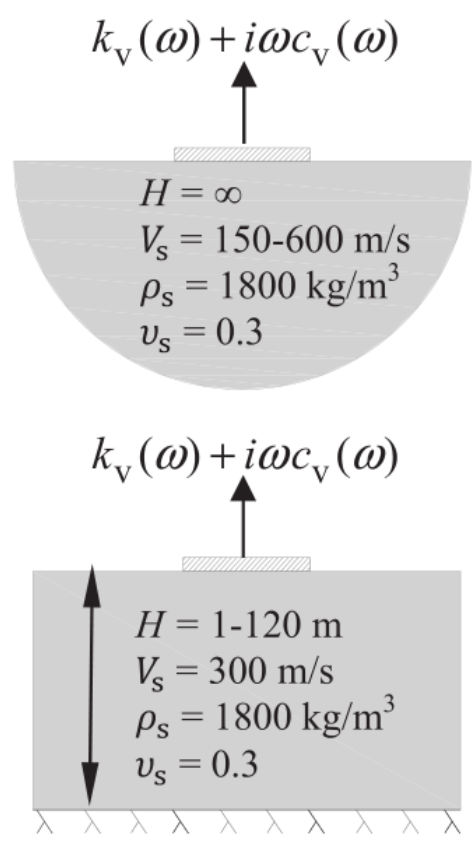

(a)

(b)

Figura 3.9: Diferentes modelos de terreno considerados. (a) Semiespacio homogéneo y (b) estrato homogéneo sobre roca. Zangeneh et al. [40]

Los resultados de rigidez y amortiguamiento adimensionales obtenidos en el estudio se encuentran en la figura 3.10.

(a)

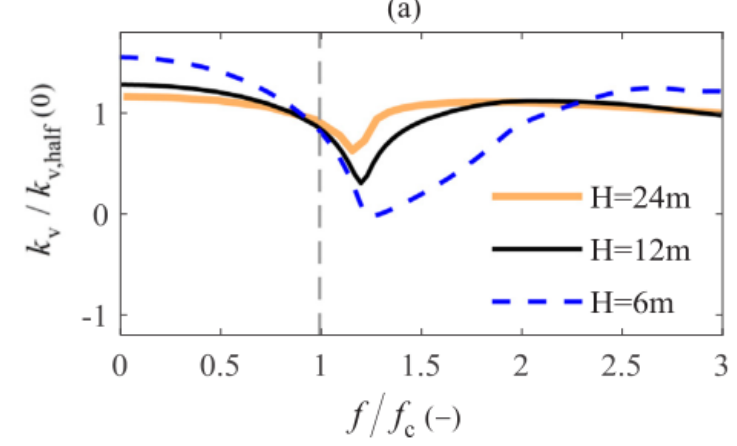

(b)

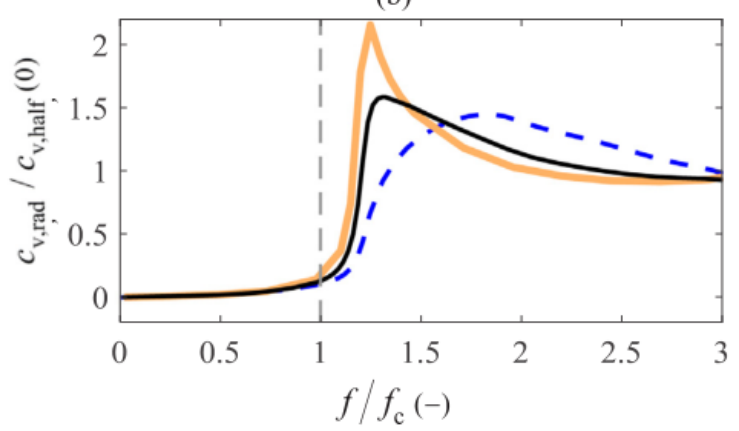

Figura 3.10: (a) Rigidez dinámica normalizada y (b) coeficiente de amortiguamiento vertical normalizado para la superficie estudiada $\left(3.5 \times 7 \mathrm{~m}^{2}\right)$, restante en estrato homogéneo con velocidad de onda $V_{s}=300 \mathrm{~m} / \mathrm{s}$ y gran $\mathrm{H}$ sobre roca. El eje de abcisas representa la relación entre la frecuencia estudiada y la de resonancia. Zangeneh et al. [40]

El valor de rigidez experimenta un descenso cuando la frecuencia es cercana a la de resonancia, más cuantioso cuanto menor es el valor de $\mathrm{H}$. Por lo que si $\mathrm{H}$ tiene un valor muy elevado se puede adoptar un valor de rigidez constante.

Por su parte el amortiguamiento presenta un comportamiento completamente diferente, aumentando en frecuencias cercana a la de resonancia y siendo mayor el aumento a medida que aumenta el valor de $\mathrm{H}$. 


\subsection{Características dinámicas de las cimentaciones mediante pilotes}

\subsubsection{Introducción}

La rigidez dinámica y el amortiguamiento son necesarios en el análisis de estructuras cimentadas mediante pilotes sometidas a cargas móviles.

Los valores de rigidez y amortiguamiento se ven afectados por la interacción con el terreno. Hace un tiempo esta interacción se consideraba mediante una viga equivalente en voladizo más corta que el pilote real. El amortiguamiento se calculaba mediante una estimación.

En estudios más recientes se entiende la interacción suelo-estructura en términos de propagación de ondas elásticas. Para los pilotes aislados se ha optado por aproximaciones continuas (Kobori et al. [8], Nogami and Novak [18], Novak [19], Novak and Aboul-Ella [22] y Tajimi [35]) y mediante elementos finitos (Blaney et al. [3], Kuhlemeyer [9] y Wolf and von Arx [39]). Los estudios citados indican que la interacción suelo-estructura provoca una alteración de la rigidez del pilote, haciéndola dependiente de la frecuencia y que genera un amortiguamiento geométrico.

En grupos de pilotes cercanos la caracterización de la rigidez y el amortiguamiento posee una mayor dificultad debido a la interacción entre pilotes o efecto de grupo. La solución para grupos de pilotes ha sido extendida por Kaynia and Kausel [7], Nogami [17], Sheta and Novak [34], Trbojevic et al. [36], Waas and Hartmann [38] y Wolf and von Arx [39]. El comportamiento dinámico de los grupos de pilotes es mucho más complicado de caracterizar porque es mucho más dependiente de la frecuencia. Sin embargo, es posible analizar grupos de pilotes en ocasiones concretas, como por ejemplo a bajas frecuencias, grupos pequeños o pilotes muy separados entre sí. Dichos cálculos se realizan mediante medias de los factores de interacción.

Los factores de interacción derivan de la consideración de dos pilotes exactamente iguales y la determinación del desplazamiento que se produce en uno si se aplica una carga en el otro. Los citados factores fueron presentados para cargas estáticas por Poulos [29] y Poulos and Davis [30] y para cargas dinámicas por Kaynia and Kausel [7], Randolph and Poulos [31] y Trbojevic et al. [36] realizaron aproximaciones al problema basadas en los factores de interacción proporcionando útiles resultados.

En los últimos años se ha incidido en publicaciones de artículos basados en el cálculo de los grupos de pilotes mediante el empleo de los factores de interacción anteriormente descritos. En Álamo et al. [10] se propone un método numérico que permite el cálculo de grupos de pilotes inclinados en suelos estratificados y en Álamo et al. [11] se describe la importancia del uso de perfiles precisos del suelo a la hora de llevar a cabo cálculos que impliquen la obtención de factores cinemáticos. 
Cuando la distancia entre pilotes es muy extensa los factores de interacción obtienen valores irrisorios, de manera que la rigidez y el amortiguamiento del grupo se obtienen sumando las características de cada pilote de forma individual (Novak [19]).

\subsubsection{Caracterización de la rigidez y el amortiguamiento dinámicos}

La ecuación del movimiento en el dominio del tiempo es la que se puede apreciar a continuación, donde v representa desplazamiento vertical.

$$
m \ddot{u}+c \dot{u}+k u=f
$$

Si a la ecuación (3.1) se le aplica la Transformada de Fourier (3.2) se produce un traslado del dominio del tiempo al de la frecuencia, siendo (3.3) la ecuación del movimiento en dicho dominio.

$$
\begin{gathered}
u=U e^{i \omega t} \\
-\omega^{2} m U+i \omega c U+k U=F
\end{gathered}
$$

De la ecuación (3.3) se extrae que en el dominio de la frecuencia la rigidez dinámica está compuesta por una parte real y una parte imaginaria. Pudiendo expresarse como se indica a continuación.

$$
K=k_{1}+i k_{2}
$$

La parte real representa la rigidez constante del pilote. La parte imaginaria representa amortiguamiento debido a la energía de disipación en suelo y pilote. Este amortiguamiento suele crecer con la frecuencia de forma que se asemeja a un amortiguamiento viscoso, pudiendo definirse en términos de una constante del mismo.

$$
c=\frac{k_{2}}{\omega}
$$

Pasando la rigidez dinámica a expresarse de la forma siguiente.

$$
K=k+i \omega c
$$

La rigidez y el amortiguamiento pueden definirse experimentalmente o de forma teórica, siendo esta última la más indicada debido a que los experimentos son de difícil generalización. 


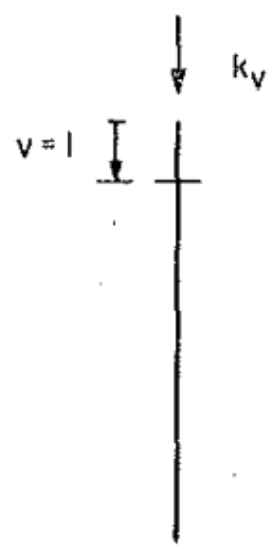

VERTICAL

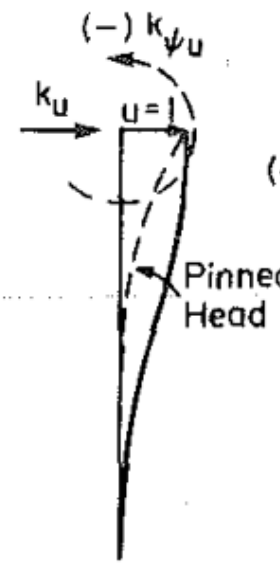

HORIZONTAL

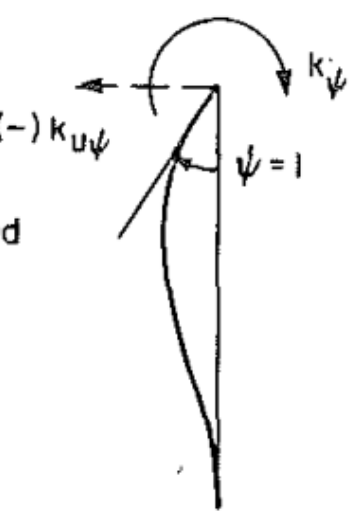

ROTATION

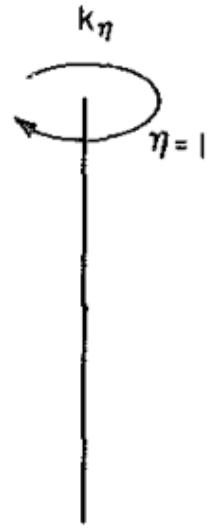

TORSION

Figura 3.11: Generación de rigidez en el pilote en direcciones individuales. Novak et al. [28]

Los valores de rigidez $(k)$ y amortiguamiento vertical (c) se obtienen del estudio de Novak [19]. A continuación se pueden observar las expresiones para cada grado de libertad.

Desplazamiento vertical, v:

$$
k_{v}=\frac{E_{p} A}{R} f_{v 1} \quad c_{v}=\frac{E_{p} A}{V_{s}} f_{v 2}
$$

Desplazamiento horizontal, $\mathrm{u}$ :

$$
k_{u}=\frac{E_{p} I}{R^{3}} f_{u 1} \quad c_{u}=\frac{E_{p} I}{R^{2} V_{s}} f_{u 2}
$$

Rotación de la cabeza del pilote en el plano vertical, $\psi$ :

$$
k_{\psi}=\frac{E_{p} I}{R} f_{\psi 1} \quad c_{\psi}=\frac{E_{p} I}{V_{s}} f_{\psi 2}
$$

Pareja de traslación horizontal y rotación, c:

$$
k_{c}=\frac{E_{p} I}{R^{2}} f_{c 1} \quad c_{u}=\frac{E_{p} I}{R V_{s}} f_{c 2}
$$

Donde $k_{c}=k_{u \psi}=k_{\psi u}$

Torsión, $\eta$ :

$$
k_{\eta}=\frac{G_{p} J}{R} f_{\eta 1} \quad c_{\eta}=\frac{G_{p} J}{V_{s}} f_{\eta 2}
$$

Los símbolos $f_{1}$ y $f_{2}$ son valores adimensionales de rigidez y amortiguamiento. Dependen de los siguientes factores. 
- Frecuencia adimensional: $a_{0}=\frac{\omega R}{V_{s}}$

- Rigidez relativa entre pilote y terreno: $E_{p} / G_{s}$

- Relación entre velocidad de compresión y cizalla en el pilote: $\bar{v}=V_{s} / v_{c}$

- Relación entre la densidad del terreno y la del pilote: $\rho / \rho_{p}$

- Esbeltez del pilote: 1/R

- Amortiguamiento del material que compone el pilote y el terreno

El significado de las variables utilizadas en las fórmulas anteriores es el siguiente:

- $\omega$ : Frecuencia angular

- $v_{c}$ : Velocidad de las ondas de compresión en el pilote $\left(v_{c}=\sqrt{E_{p} / \rho_{p}}\right)$

- $V_{s}$ : Velocidad de ondas de cizalla en el terreno $\left(V_{s}=\sqrt{G_{s} / \rho}\right)$

- $\rho$ : Densidad del terreno

- $\rho_{p}$ : Densidad del material que compone el pilote

- $E_{p}$ : Módulo de Young del pilote

- $G_{p} J$ : Rigidez torsional

- $G_{s}$ : Módulo de cizalla del suelo

- $V_{s}$ : Velocidad de las ondas de cizalla en el suelo

- R: Radio del pilote

- 1: longitud del pilote

- A: Área del pilote

- I: Momento de inercia del pilote

Para una correcta consideración de las variables indicadas con anterioridad es necesario el uso de la mecánica computacional. Un método eficiente y para cualquier clase de terreno fue elaborado por Novak y Aboul-Ella (Novak and Aboul-Ella [21], Novak and Aboul-Ella [22] y Novak and Grigg [23]). El método fue generalizado para incluir la separación entre pilotes por Novak and Sheta [26]. Estas soluciones están basadas en la hipótesis de deformación plana del terreno Novak et al. [27].

Las formulaciones anteriores son una aproximación, pero si se comparan con con las soluciones mediante el cálculo con elementos finitos presentadas por Blaney et al. [3], Kuhlemeyer [9] y Kaynia and Kausel [6] se demuestra que la hipótesis de deformación plana proporciona soluciones suficientemente precisas, sobre todo a altas frecuencias. Para bajas frecuencias la solución puede sufrir una corrección.

El método descrito en Novak and Aboul-Ella [21] y Novak and Aboul-Ella [22] fue el utilizado para la elaboración de Novak et al. [28], artículo en el que se basa este trabajo.

Los efectos de la frecuencia adimensional pueden observarse en la figura 3.12, extraída del trabajo de Nogami and Novak [18]. 


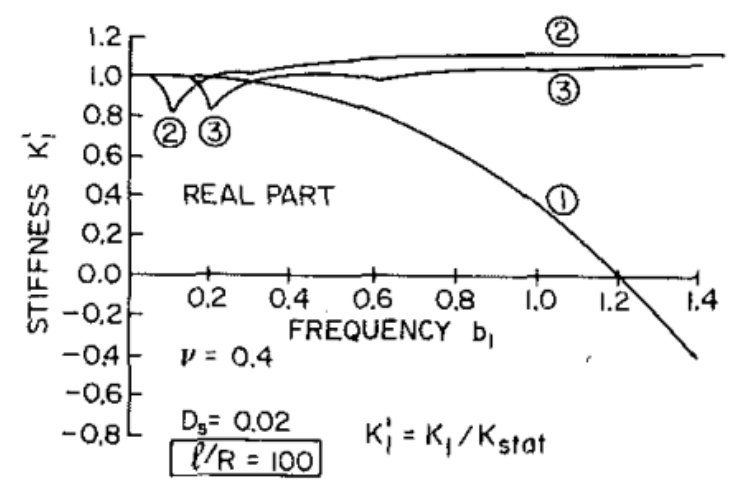

(a)

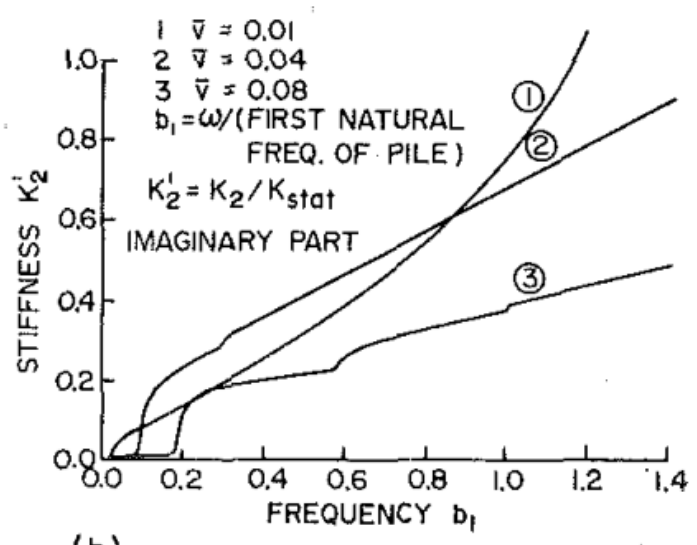

(b)

Figura 3.12: Ejemplo de variación de la rigidez de la pila en función de la frecuencia y la rigidez del terreno. Nogami and Novak [18]

En la figura 3.12 se puede observar una relación entre la frecuencia dinámica (parte real e imaginaria) y la frecuencia estática.

La parte real de la rigidez disminuye de manera considerable con el aumento de la frecuencia, pudiendo incluso llegar a ser negativa si el terreno es muy flexible o el pilote demasiado robusto. Sin embargo, para pilas más esbeltas en suelos más habituales el valor de la parte real se mantiene prácticamente constante para cualquier valor de frecuencia. Los pequeños mínimos que se pueden obervar en las curvas 2 y 3 de la figura 3.12 se deben a efectos de resonancia que desaparecen para valores de amortiguamiento del material más altos.

La parte imaginaria de la rigidez (amortiguamiento de la pila) crece de manera casi lineal con la frecuencia, de manera que puede expresarse a partir de una constante de amortiguamiento viscoso, c, que es casi independiente de la frecuencia. Solamente por debajo de la frecuencia natural del terreno, indicada por los mínimos en la gráfica 3.12 el amortiguamiento geométrico disminuye y el propio del material es el dominante. Se puede esperar que en bajas frecuencias, terrenos muy rígidos y suelos muy someros el amortiguamiento sea prácticamente inexistente.

De la figura 3.12 y lo explicado con posterioridad a ella se extrae una conclusión muy importante, que es sobre la que se basa completamente la realización del presente trabajo. En las cimentaciones profundas mediante pilotes, salvo caso muy excepcionales, los valores de rigidez (k) y amortiguamiento (c) son constantes, lo que permite el cálculo del amortiguamiento geométrico trabajo en el dominio del tiempo con independencia de la frecuencia.

La relación entre la densidad del terreno y la del pilote $\left(\rho / \rho_{p}\right)$ es otro factor que solamente interviene en casos extremos, como puede observarse en la figura 3.13. La rigidez y amortiguamiento de la pila solo sufren variación si el pilote posee una masa muy elevada. 


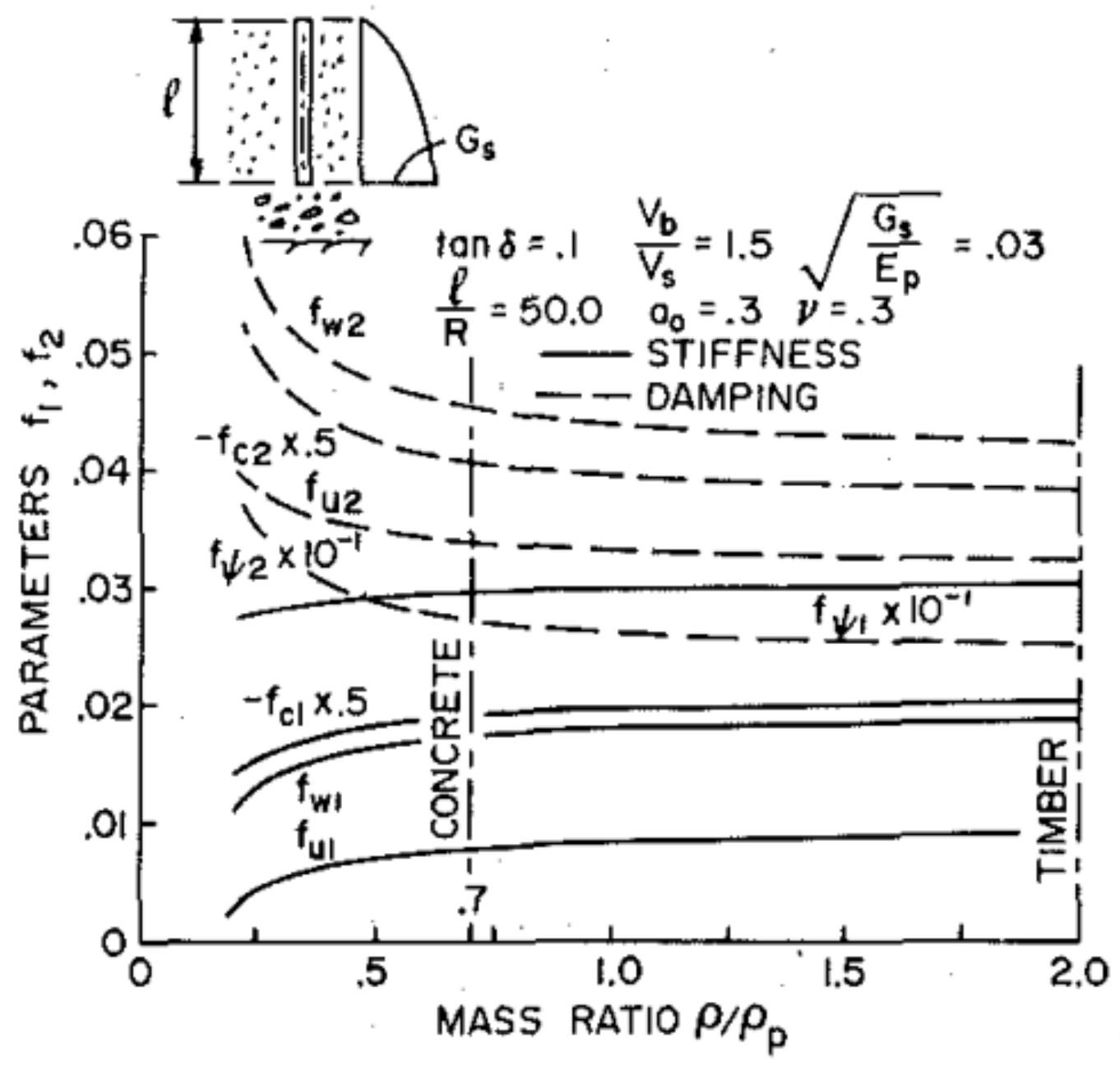

Figura 3.13: Efecto de la relación entre las masas del pilote y el terreno en los parámetros de rigidez y amortiguamiento. Novak and Aboul-Ella [21]

El efecto del módulo de Poisson es muy débil para la vibración vertical, ausente para la torsión y no demasiado importante para el resto de modos de vibración, excepto si su valor es muy próximo a 0.5 y las freuencias son altas. El efecto del módulo de Poisson se puede reducir si la relación de rigideces entre el pilote y el terreno se calcula de la forma $E_{p} / E_{s}$ en lugar de con $E_{p} / G_{s}$ (Roesset [32]).

La esbeltez $(1 / R)$ y las condiciones de empotramiento en punta son muy importantes para pilotes cortos y en la dirección vertical, donde los pilotes son rígidos. En la dirección horizontal los pilotes son muy flexibles y los factores $f_{1}$ y $f_{2}$ son independientes de la esbeltez y las condiciones en punta para relaciones $1 / R$ mayores a 25 y terrenos homogéneos (Novak [19] y Novak [20]). Si la rigidez del suelo disminuye en la sentido vertical ascendente los factores $f_{1}$ y $f_{2}$ disminuyen a mayores esbelteces.

De lo explicado anteriormente se extrae que los factores que que poseen una mayor influencia en el cálculo de $f_{1}$ y $f_{2}$ son: 
- La relación entre la rigidez del pilote y la del suelo

- El perfil del terreno

- La esbeltez y las condiciones en punta

\subsubsection{Gráficas de rigidez y amortiguamiento}

Dirección horizontal

En la figura 3.14 se pueden observar los valores $f_{1}$ y $f_{2}$ asociados a vibración horizontal en la cabeza de los pilotes.

\begin{tabular}{|c|c|c|c|c|c|c|c|c|c|}
\hline \multirow[b]{2}{*}{$\begin{array}{c}v \\
(1) \\
\end{array}$} & \multirow{2}{*}{$\begin{array}{c}E_{\text {pile }} / \\
G_{\text {soll }} \\
(2)\end{array}$} & \multicolumn{4}{|c|}{ Stiffness Parameters } & \multicolumn{4}{|c|}{ Damping Parameters } \\
\hline & & $\begin{array}{l}f_{\phi 1} \\
(3)\end{array}$ & $\begin{array}{l}f_{d} \\
(4)\end{array}$ & $\begin{array}{l}f_{u 1} \\
(5)\end{array}$ & $\begin{array}{l}f_{h}^{p_{1}} \\
(6)\end{array}$ & $\begin{array}{l}f_{v 2} \\
(7)\end{array}$ & $\begin{array}{l}f_{62} \\
(8)\end{array}$ & $\begin{array}{l}f_{u 2} \\
(9)\end{array}$ & $\begin{array}{l}f_{b_{2}}^{p} \\
(10)\end{array}$ \\
\hline \multicolumn{10}{|c|}{ (a) Homogeneous Soil Profile } \\
\hline \multirow[t]{5}{*}{0.25} & 10,000 & 0.2135 & -0.0217 & 0.0042 & 0.0021 & 0.1577 & -0.0333 & 0.0107 & 0.0054 \\
\hline & 2,500 & 0.2998 & -0.0429 & 0.0119 & 0.0061 & 0.2152 & -0.0646 & 0.0297 & 0.0154 \\
\hline & 1,000 & 0.3741 & -0.0668 & 0.0236 & 0.0123 & 0.2598 & -0.0985 & 0.0579 & 0.0306 \\
\hline & 500 & 0.4411 & -0.0929 & 0.0395 & 0.0210 & 0.2953 & -0.1337 & 0.0953 & 0.0514 \\
\hline & 250 & 0.5186 & -0.1281 & 0.0659 & 0.0358 & 0.3299 & -0.1786 & 0.1556 & 0.0864 \\
\hline \multirow[t]{5}{*}{0.40} & 10,000 & 0.2207 & -0.0232 & 0.0047 & 0.0024 & 0.1634 & -0.0358 & 0.0119 & 0.0060 \\
\hline & 2,500 & 0.3097 & -0.0459 & 0.0132 & 0.0068 & 0.2224 & -0.0692 & 0.0329 & 0.0171 \\
\hline & 1,000 & 0.3860 & -0.0714 & 0.0261 & 0.0136 & 0.2677 & -0.1052 & 0.0641 & 0.0339 \\
\hline & 500 & 0.4547 & -0.0991 & 0.0436 & 0.0231 & 0.3034 & -0.1425 & 0.1054 & 0.0570 \\
\hline & 250 & 0.5336 & -0.1365 & 0.0726 & 0.0394 & 0.3377 & -0.1896 & 0.1717 & 0.0957 \\
\hline \multicolumn{10}{|c|}{ (b) Parabolic Soil Profile } \\
\hline \multirow[t]{5}{*}{0.25} & 10,000 & 0.1800 & -0.0144 & 0.0019 & 0.0008 & 0.1450 & -0.0252 & 0.0060 & 0.0028 \\
\hline & 2,500 & 0.2452 & -0.0267 & 0.0047 & 0.0020 & 0.2025 & -0.0484 & 0.0159 & 0.0076 \\
\hline & 1,000 & 0.3000 & -0.0400 & 0.0086 & 0.0037 & 0.2499 & -0.0737 & 0.0303 & 0.0147 \\
\hline & 500 & 0.3489 & -0.0543 & 0.0136 & 0.0059 & 0.2910 & -0.1008 & 0.0491 & 0.0241 \\
\hline & 250 & 0.4049 & -0.0734 & 0.0215 & 0.0094 & 0.3361 & -0.1370 & 0.0793 & 0.0398 \\
\hline \multirow[t]{5}{*}{0.40} & 10,000 & 0.1857 & -0.0153 & 0.0020 & 0.0009 & 0.1508 & -0.0271 & 0.0067 & 0.0031 \\
\hline & 2,500 & 0.2529 & -0.0284 & 0.0051 & 0.0022 & 0.2101 & -0.0519 & 0.0177 & 0.0084 \\
\hline & 1,000 & 0.3094 & -0.0426 & 0.0094 & 0.0041 & 0.2589 & -0.0790 & 0.0336 & 0.0163 \\
\hline & 500 & 0.3596 & -0.0577 & 0.0149 & 0.0065 & 0.3009 & -0.1079 & 0.0544 & 0.0269 \\
\hline & 250 & 0.4170 & -0.0780 & 0.0236 & 0.0103 & 0.3468 & -0.1461 & 0.0880 & 0.0443 \\
\hline
\end{tabular}

Figura 3.14: Parámetros de rigidez y amortiguamiento para vibración horizontal de pilotes con $l / R>25$ en perfiles homogéneos y $l / R>30$ en suelos de perfil parabólico. Novak et al. [28]

Comparando los resultados de la figura 3.14 se extrae que los suelos de perfil parabólico producen valores menores de rigidez y amortiguamiento. En la figura aparecen expresiones de la forma $f_{u 1}^{p} y f_{u 2}^{p}$ que corresponden a pilotes apoyados en cabeza. La rigidez y el amortiguamiento de dichos pilotes es menor que los que poseen los empotrados en cabeza.

\section{Dirección vertical}

Los valores de rigidez y amortiguamiento son fuertemente dependientes de la esbeltez y las condiciones en punta del pilote. En las figuras 3.15 y 3.16 se pueden observar los valores de $f_{v 1}$ y $f_{v 2}$ para varias relaciones de esbeltez y de rigidez del suelo. Los resultados se aportan en dos gráficas según la tipología del pilote en punta, flotantes o empotrados. Dentro de cada tipología de pilote se distingue entre terreno rigidez homogénea y de creciente de forma parabólica en el sentido vertical descendente. 

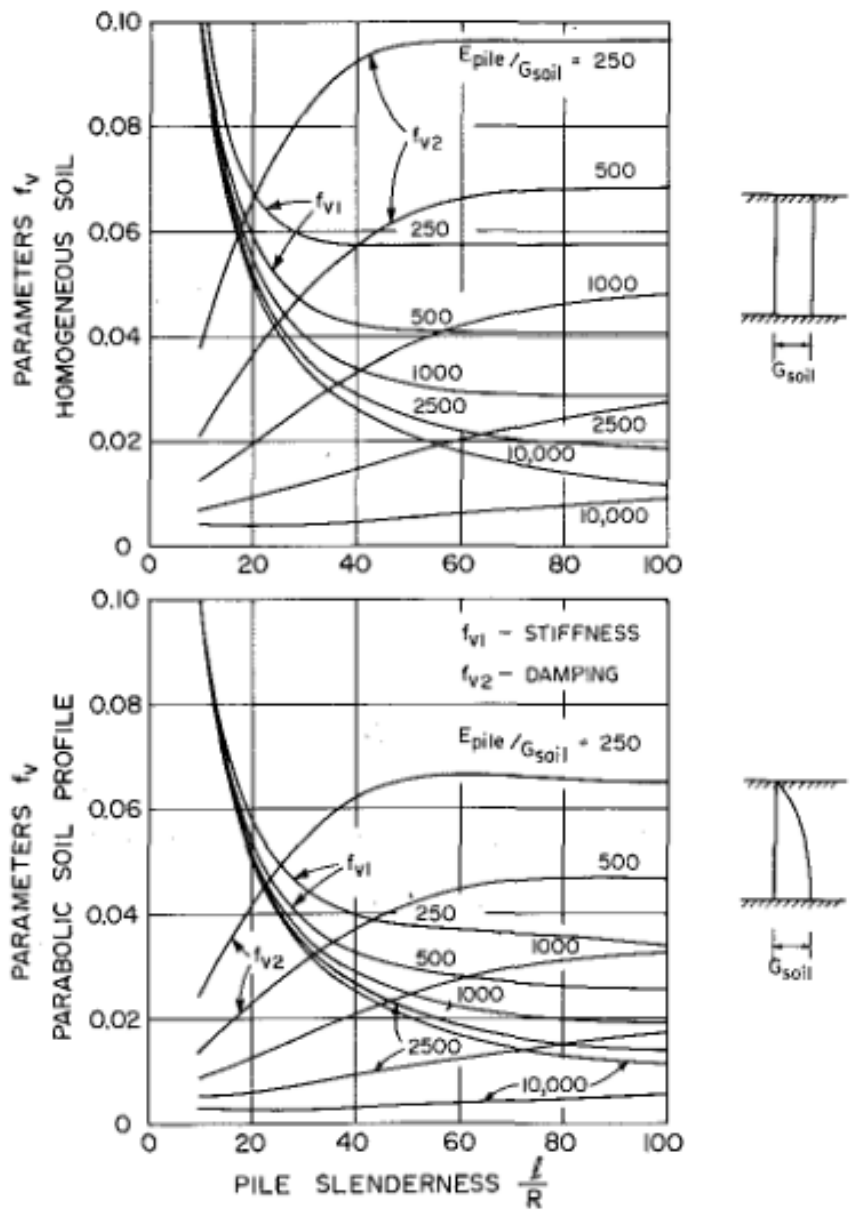

Figura 3.15: Valores de $f_{v 1}$ y $f_{v 2}$ para pilotes empotrados. Novak et al. [28] 


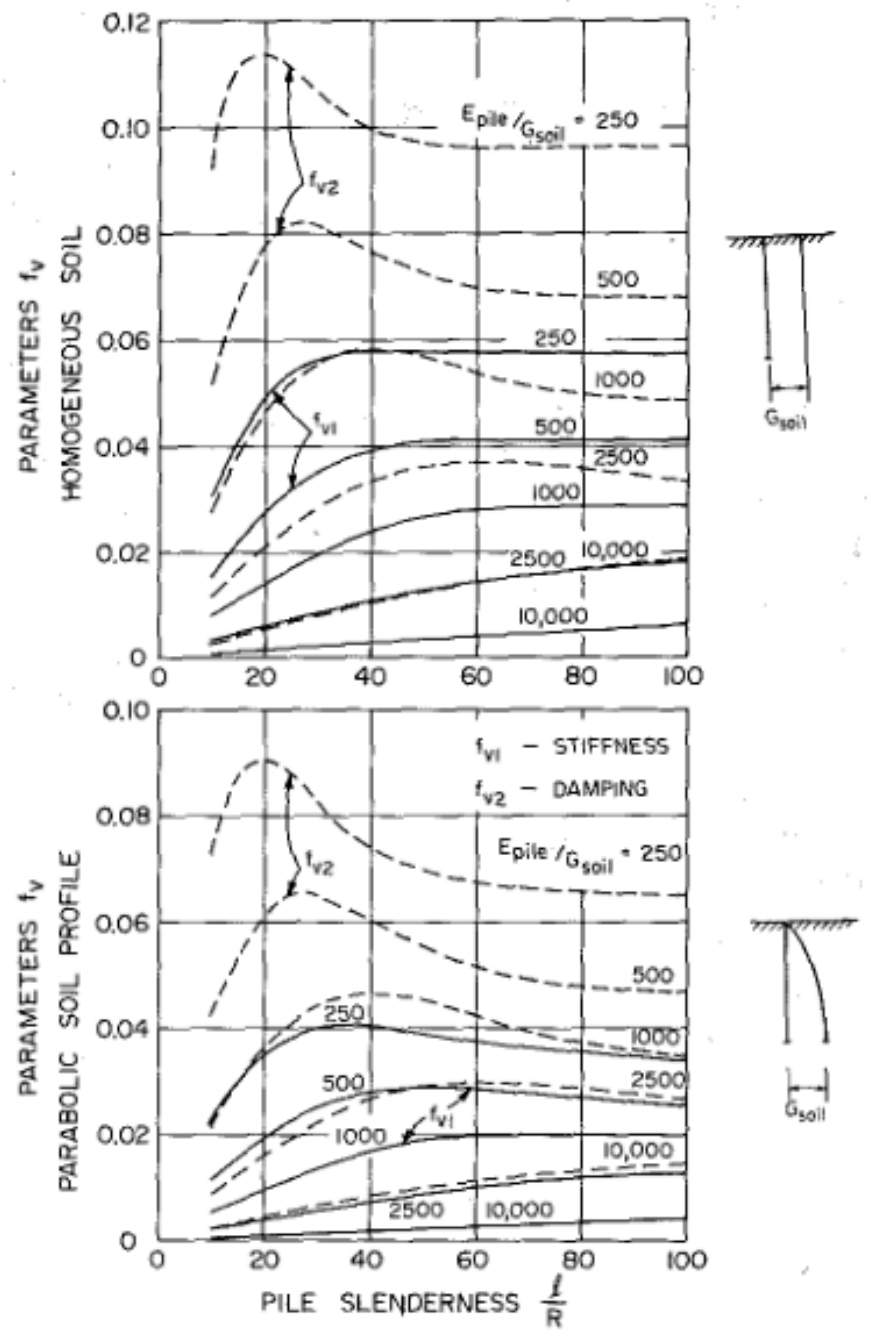

Figura 3.16: Valores de $f_{v 1}$ y $f_{v 2}$ para pilotes flotantes. Novak et al. [28]

En las gráficas se puede apreciar como los valores de rigidez y amortiguamiento son menores para el suelo de perfil parabólico que para el homogéneo. En cuanto a la tipología de pilote se puede observar como los pilotes flotantes experimentan un mayor amortiguamiento y una rigidez mucho menor en las esbelteces más pequeñas.

La torsión es muy dependiente de la frecuencia. Se pueden consultar parámetros torsionales en Novak and Howell [24] y Novak and Howell [25].

\subsubsection{Conclusiones}

Del trabajo de Novak et al. [28] se extraen las siguientes conclusiones:

- Los pilotes apoyados en cabeza poseen menos rigidez y amortiguamiento que los empotrados.

- La variación parabólica de la rigidez del terreno proporciona unos valores de rigidez y amortiguamiento mucho menores 
- Los pilotes flotantes generan en la dirección vertical un mayor amortiguamiento pero una menor rigidez.

\subsection{Análisis modal en pérgolas cimentadas por pilotes}

La expresión que gobierna el movimiento de un sistema de múltiples grados de libertad sin amortiguamiento es la indicada en la ecuación (3.12).

$$
M \ddot{u}+K u=0
$$

La modos de vibración son vectores ortogonales, lo que los hace linealmente independientes. Que los modos de vibración formen una base de vectores linealmente independientes permite expresar el desplazamiento sufrido mediante una combinación lineal de los mismos.

Una vez explicado lo anterior, a la ecuación (3.12) se le aplica el cambio de variable $u(t)=$ $\Phi q(t)$, siendo $\Phi$ y $\mathrm{q}(\mathrm{t})$ la matriz y contribución modal respectivamente, y se multiplica por la matriz traspuesta de la modal se obtiene lo indicado en 3.13 .

$$
\Phi^{T} M \Phi \ddot{q}+\Phi^{T} K \Phi q=0
$$

La matriz modal es ortogonal respecto a la matriz de masa y la de rigidez, dicho lo cual el sistema de múltiples grados de libertad se desacopla en múltiples sistemas de un grado de libertad, notándose el modo correspondiente como i.

$$
m_{i} \ddot{q}_{i}+k_{i} q_{i}=0
$$

La expresión (3.14) es equivalente a la (3.15).

$$
\ddot{q}_{i}+\omega_{i}^{2} q_{i}=0
$$

Si la ecuación (3.15) se enriquece con la inclusión de una tasa de amortiguamiento se obtiene la expresión (3.16).

$$
\ddot{q}_{i}+2 \xi_{i} \omega_{i} \dot{q}_{i}+\omega_{i}^{2} q_{i}=0
$$

La tasa de amortiguamiento $(\xi)$ está compuesta por los siguientes términos:

1. Amortiguamiento estructural: Viene definido en la normativa IAPF-11 [2]. Para estructuras de hormigón se debe tomar una tasa del $2 \%$. 
2. Amortiguamiento geométrico: Viene definido por la interacción entre la estructura y el terreno que se encuentra bajo ella. Se calcula a partir de la matriz de amortiguamiento (D), tal y como se explica a continuación.

La estructura tiene definidos unos sistemas de un grado de libertad (vertical) en una serie de puntos finitos, que son cada uno de los pilotes que componen la cimentación. Esto provoca que la matriz D solo tenga un valor distinto del nulo en el grado de libertad correspondiente al movimiento vertical, siendo ese valor igual al amortiguamiento del sistema (c).

$$
\Phi^{T} D \Phi=\sum_{j=1}^{j=n^{\mathrm{o}} \text { pilotes }} \phi_{i j}^{2} c
$$

La expresión obtenida en la ecuación (3.17) es equivalente a la parte de la ecuación (3.16) que representa el amortiguamiento.

$$
\sum_{j=1}^{j=n^{\mathrm{o}} \text { pilotes }} \phi_{i j}^{2} c=2 \xi_{i} \omega_{i}
$$

El valor de $\phi_{i}$ corresponde al desplazamiento vertical obtenido en cada uno de los pilotes para cada modo de vibración, obtándose a partir de este momento por notarlo como $v_{i}$. Si se procede a despejar el valor de $\xi$ de la ecuación (3.18) es posible obtener una expresión análitica para el cálculo de la tasa de amortiguamiento geométrico.

$$
\xi_{i}=\frac{\sum_{j=1}^{j=n^{\mathrm{o}} \text { pilotes }} v_{i j}^{2} c}{2 \omega_{i}}
$$

Como se ha explicado con anterioridad, en las cimentaciones mediante pilotes el valor de la rigidez ( $\mathrm{k}$ ) y del amortiguamiento (c) son constantes para todo valor de frecuencia, por lo que existe la posibilidad de calcular la tasa de amortiguamiento geométrico para cada modo de vibración en el dominio del tiempo mediante la fórmula (3.19). 


\section{CAPÍtulo 4}

\section{Pérgola de Bobadilla II}

Para el desarrollo de este documento se va a trabajar sobre el modelo de una pérgola real, que cumple los estados límite últimos y de servicio estipulados en la normativa IAPF-2011 [2]. El propósito de esta acción es que los resultados extraídos de este trabajo sean aplicables al resto de pérgolas construidas a lo largo de la extensa red de alta velocidad española, así como a los futuros diseños de las mismas.

\subsection{Geometría de la pérgola de estudio}

La pérgola de estudio es la de Bobadilla II, perteneciente a la línea de alta velocidad Antequera - Granada y diseñada por ACL-Estructuras. En las figura 4.1 se puede observar su planta, y en la figura 4.2 la tipología de las vigas que la integran.

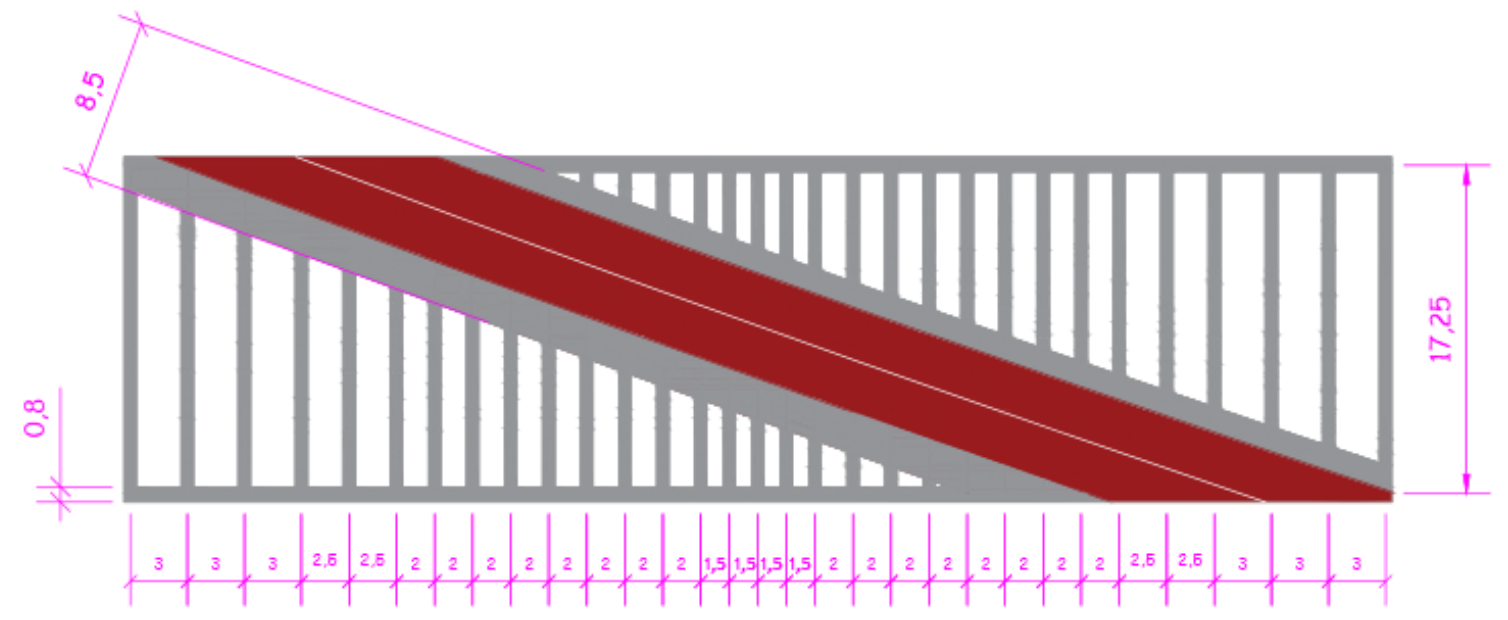

Figura 4.1: Planta. Pérgola Bobadilla II 


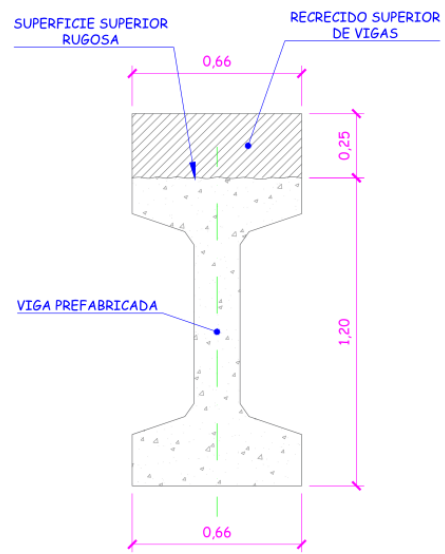

Figura 4.2: Tipología de vigas. Pérgola Bobadilla II

La pérgola está compuesta por los siguientes elementos estructurales. Entre los mismos se incluyen vigas de hormigón armado y pretensado y una losa de hormigón armado.

A continuación se pueden apreciar las características de los elementos estructurales indicados:

- Vigas de hormigón pretensado HP-50

- 31 vigas de $1.20 \mathrm{~m}$ de canto

- $\mathrm{E}=35 \mathrm{GPa}$

- $v=0.2$

- $\rho=2500 \frac{\mathrm{kg}}{\mathrm{m}^{3}}$

- Viga de Bernoulli-Euler

- Losa de hormigón armado HA-30

- $\mathrm{E}=30 \mathrm{GPa}$

- $v=0.2$

- $\rho=2500 \mathrm{~kg} / \mathrm{m}^{3}$

- Placa de Kirchhoff-Love

- Viga riostra de hormigón armado HA-30

- $\mathrm{E}=30 \mathrm{GPa}$

- $v=0.2$

- $\rho=2500 \mathrm{~kg} / \mathrm{m}^{3}$

- Viga de Bernoulli-Euler

La cimentación consiste en un elemento unidimensional formado por la unión del fuste, la zapata y un pilote de $30 \mathrm{~m}$. En la figura 4.3 se puede observar la cimentación en detalle. 


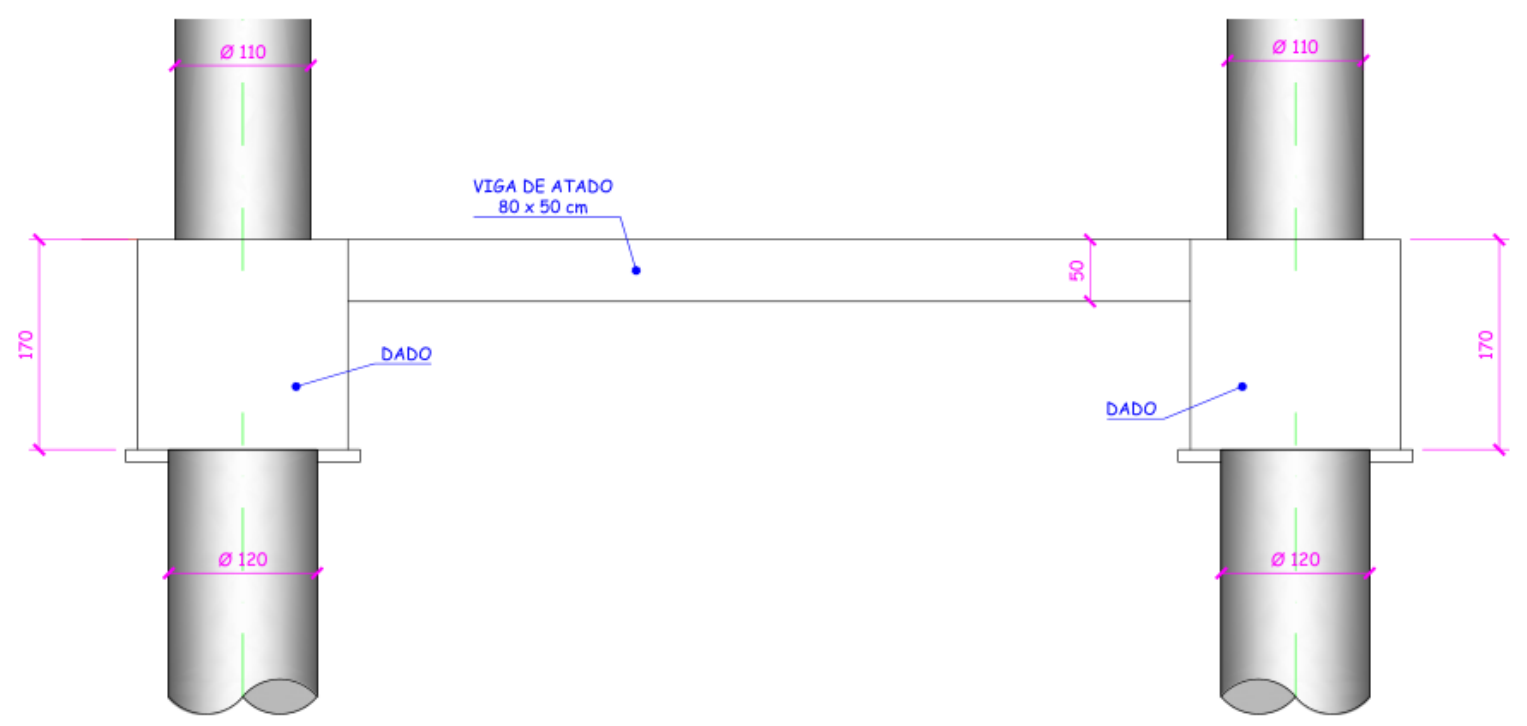

Figura 4.3: Tipología de cimentación. Pérgola de Bobadilla II

\subsection{Modelo tridimensional de la pérgola}

El modelo de la pérgola se realiza en el programa SAP2000. Se modelan las vigas y se unen a la losa superior y entre sí con una viga riostra. Como se ha comentado anteriormente, tanto en las vigas como en la placa se desprecia la deformación por cortante.

Se colocan dos apoyos que restringen el movimiento vertical en los extremos de la línea de carga, evitando una flecha que no existe en la realidad ya que existe continuidad con la vía antes y después de la losa.

En la figura 4.4 se puede observar la geometría del modelo en SAP2000.

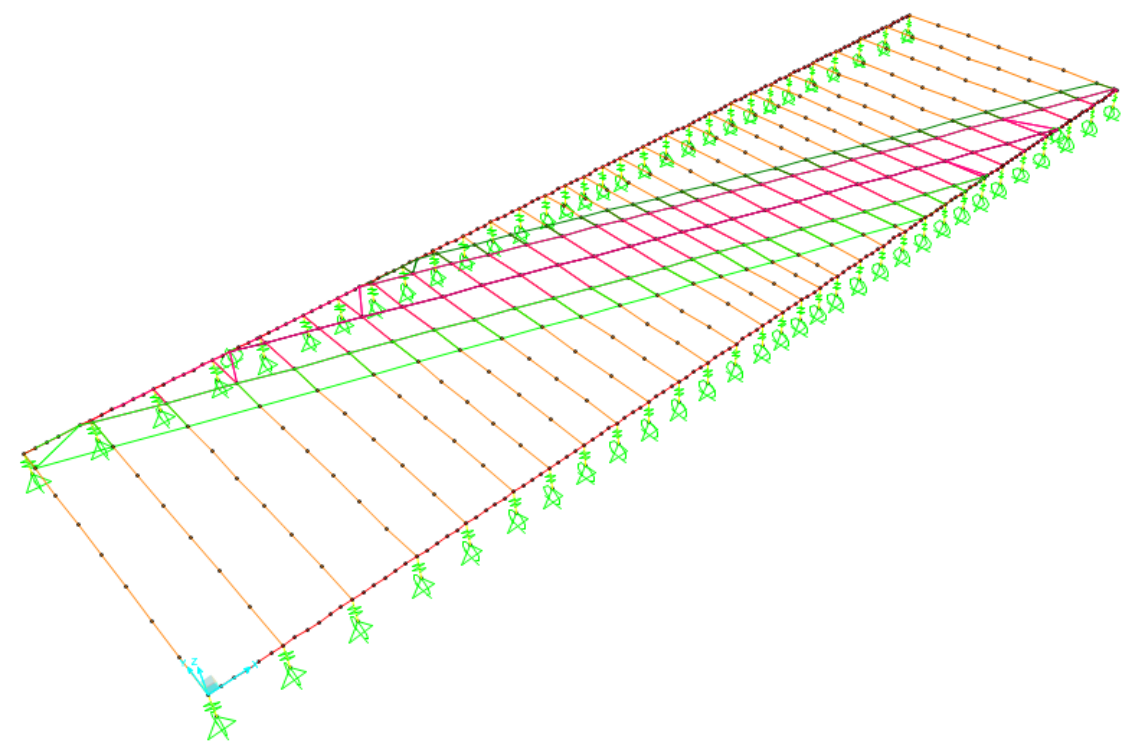

Figura 4.4: Modelo en SAP2000. Pérgola de Bobadilla II 


\subsubsection{Cargas muertas}

Las cargas muertas se van a añadir al modelo como un modificador de masa de la losa.

En la figura 4.5 se puede observar la sección transversal de la vía, de la que se obtienen el conjunto de cargas muertas que actúan sobre la estructura.

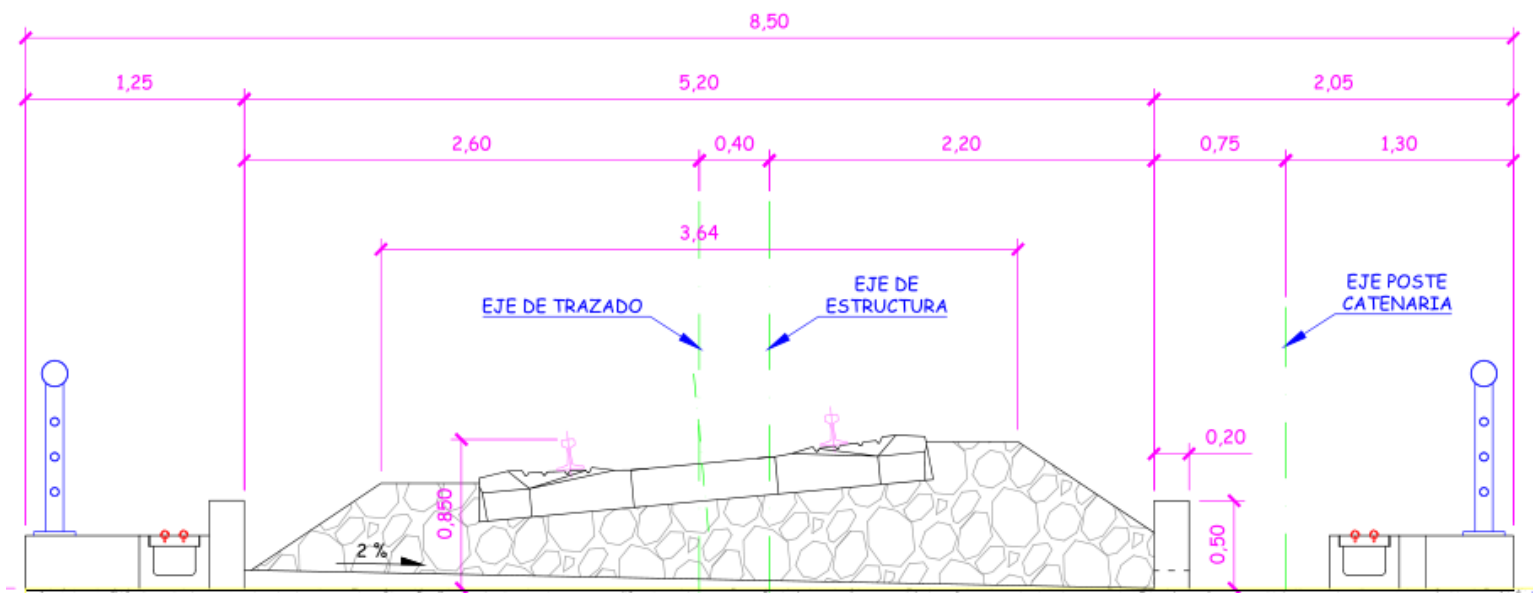

Figura 4.5: Sección transversal de la vía. Pérgola de Bobadilla II

A continuación se obtiene el coeficiente multiplicador de masa para cada una de las tres zonas en que está dividida la losa. Para ello en primer lugar se obtendrá la densidad lineal de cada elemento que suponga una carga muerta en la estructura y se procederá a calcular la relación entre la masa existente y la masa de la losa de hormigón en solitario.

Zona izquierda de la losa

Sobre esta zona actúan las siguientes cargas muertas:

- Losa de hormigón

$\rho_{l}=2500 \mathrm{~kg} / \mathrm{m}^{3} \cdot 0.25 \mathrm{~m} \cdot 1.25 \mathrm{~m}=781.25 \mathrm{~kg} / \mathrm{m}$

- Acera

$\rho_{l}=2500 \mathrm{~kg} / \mathrm{m}^{3} \cdot 0.3 \mathrm{~m} \cdot 1 \mathrm{~m}=750 \mathrm{~kg} / \mathrm{m}$

- Pretil

$\rho_{l}=1000 \mathrm{~kg} / \mathrm{m}$

$$
\text { Coeficiente }=\frac{781.25+750+100}{781.25}=2.1
$$

Zona derecha de la losa

Sobre esta zona actúan las siguientes cargas muertas: 
- Losa de hormigón

$\rho_{l}=2500 \mathrm{~kg} / \mathrm{m}^{3} \cdot 0.25 \mathrm{~m} \cdot 2.05 \mathrm{~m}=1281.25 \mathrm{~kg} / \mathrm{m}$

- Acera

$\rho_{l}=2500 \mathrm{~kg} / \mathrm{m}^{3} \cdot 0.3 \mathrm{~m} \cdot 1 \mathrm{~m}=750 \mathrm{~kg} / \mathrm{m}$

- Pretil

$\rho_{l}=1000 \mathrm{~kg} / \mathrm{m}$

$$
\text { Coeficiente }=\frac{1281.25+750+100}{1281.25}=1.66
$$

Zona central

Sobre esta zona actúan las siguientes cargas muertas:

- Losa de hormigón

$\rho_{l}=2500 \mathrm{~kg} / \mathrm{m}^{3} \cdot 0.25 \mathrm{~m} \cdot 5.20 \mathrm{~m}=3250 \mathrm{~kg} / \mathrm{m}$

- Balasto

Acorde a las indicaciones de la legislación vigente en materia de cargas ferroviarias se asigna al balasto una densidad de $1800 \mathrm{~kg} / \mathrm{m}^{3}$ y un espesor de $0,5 \mathrm{~m}$.

$\rho_{l}=1800 \mathrm{~kg} / \mathrm{m}^{3} \cdot 0.5 \mathrm{~m} \cdot 5.20 \mathrm{~m}=4680 \mathrm{~kg} / \mathrm{m}$

- Traviesas

Se suponen colocadas cada $0,6 \mathrm{~m}$.

$\rho_{l}=2500 \mathrm{~kg} / \mathrm{m}^{3} \cdot 0.3 \mathrm{~m} \cdot 2.60 \mathrm{~m} \cdot 0.6=2340 \mathrm{~kg} / \mathrm{m}$

- Carril

$\rho_{l}=60 \mathrm{~kg} / \mathrm{m} \cdot 2=120 \mathrm{~kg} / \mathrm{m}$

- Muretes para soportar el balasto

$\rho_{l}=2500 \mathrm{~kg} / \mathrm{m}^{3} \cdot 0.2 \mathrm{~m} \cdot 0.5 \mathrm{~m} \cdot 2=500 \mathrm{~kg} / \mathrm{m}$

$$
\text { Coeficiente }=\frac{3250+4680+2340+120+500}{3250}=1.66
$$

\subsubsection{Conexión vigas - losa}

La unión entre las vigas y la losa debe hacerse de forma monolítica para poder realizar el análisis en 6 grados de libertad. Para ello el modelo se realiza en el plano del centro de 
gravedad de la losa, y posteriormente se les asigna a las vigas un offset vertical de forma que su cara superior coincida con la cara inferior de la losa.

En la figura 4.6 se puede observar la conexión en detalle.

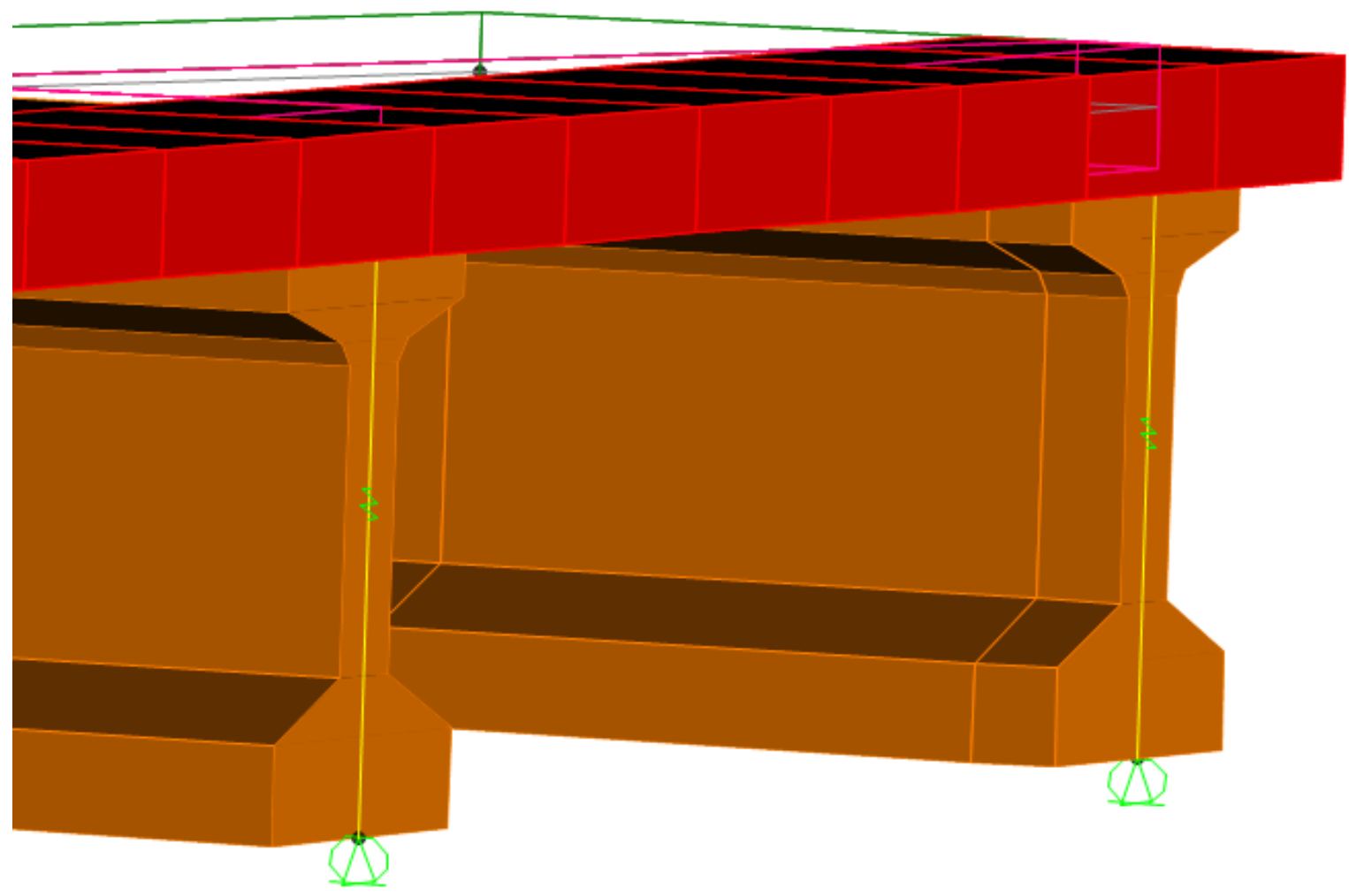

Figura 4.6: Detalle de la conexión entre las vigas y la losa. Pérgola de Bobadilla II

\subsubsection{Apoyos}

Los apoyos deben colocarse en el lugar en el que se encuentran en la realidad con el fin de no restringir los movimientos de membrana de la losa. Para ello se crea un link desde el centro de gravedad de la losa (plano del modelo) a la parte inferior de la viga, tal y como puede apreciarse en la figura 4.6. La tipología del link debe transmitir solamente los movimientos los ejes globales $x$ y $z$.

En cuanto a la tipología del apoyo se debe distinguir entre dos tipos de modelos:

- Modelo sin interacción entre el suelo y la estructura

En este modelo se restringe el movimiento en el eje $x$ y el eje $z$.

- Modelo con interacción entre el suelo y la estructura

En este modelo, mediante un link se restringe el movimiento en el eje $x$. En el eje vertical se crea un conjunto muelle + amortiguador de un grado de libertad con la masa del conjunto 
fuste + zapata + pilote y la rigidez y factor de amortiguamiento del terreno, tal y como puede observarse en la figura 4.7.

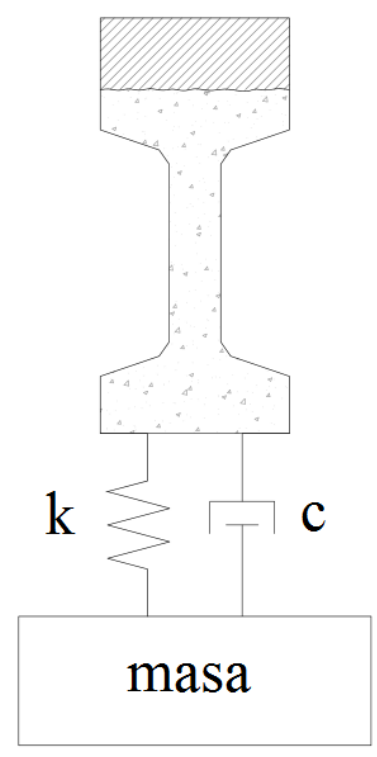

Figura 4.7: Detalle del sistema muelle - amortiguador de un grado de libertad. Pérgola de Bobadilla II

\subsection{Análisis dinámico de la pérgola de Bobadilla II}

En este apartado se va a realizar una análisis dinámico de la pérgola de Bobadilla II, con el fin de comprobar la influencia de la interacción suelo - estructura en las aceleraciones y desplazamientos máximos que experimenta la pérgola. Una vez obtenidos los datos anteriormente citados se procederá a elaborar unas gráficas que ayuden a entender el comportamiento de las estructuras de este tipo a medida que la rigidez del suelo varía.

Como se ha explicado con anterioridad, para modelizar la tipología de cimentación se emplean sistemas muelle - amortiguador de un grado de libertad en la dirección vertical, como puede observarse en la figura 4.7. Los valores de rigidez y amortiguamiento dinámicos a incluir en el sistema de un grado de libertad se obtienen a partir de los resultados del trabajo de Novak et al. [28].

El cálculo se va a realizar para la siguiente serie de relaciones entre la rigidez del pilote y la del terreno.

- $\operatorname{Apoyos}\left(E_{p} / G_{s}=0\right)$

- $E_{p} / G_{s}=250$

- $E_{p} / G_{s}=500$

- $E_{p} / G_{s}=1000$

- $E_{p} / G_{s}=2500$

- $E_{p} / G_{s}=5000$ 
- $E_{p} / G_{s}=10000$

La pérgola de Bobadilla II está diseñada para una velocidad de paso de $200 \mathrm{~km} / \mathrm{h}$ y según se indica en la normativa pertinente [2] se debe realizar el análisis dinámico hasta una velocidad un $20 \%$ superior a la estipulada para el paso de los trenes.

$$
v_{\max }=200 \mathrm{~km} / \mathrm{h} \cdot 1.2=240 \mathrm{~km} / \mathrm{h}
$$

La red debe garantizar la compatibilidad con la red europea, así que los trenes a considerar van a ser los siguientes:

- 10 trenes de la composición HSLM [1]

- Tren AVE

- Tren TALGO

En lo que respecta a los puntos de posproceso se distingue entre dos tipos:

- Puntos de posproceso de aceleración

- Puntos de posproceso de desplazamiento.

Puntos de posproceso de aceleración

Están localizados en centros y cuartos de vano de la línea de carga y los extremos de la cama de balasto, lugar donde suelen experimentarse las máximas aceleraciones en estructuras de este tipo. Son un total de 9 puntos, cuya localización puede apreciarse en la figura 4.8 .

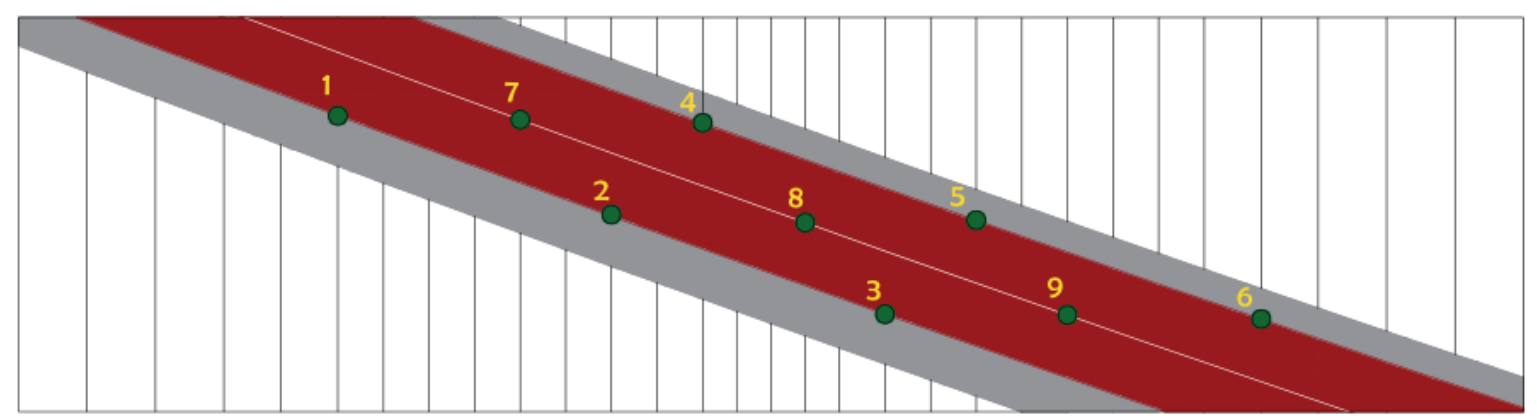

Figura 4.8: Puntos de posproceso de valores de aceleración. Pérgola de Bobadilla II

Puntos de posproceso de desplazamiento

Están localizados en centro y cuartos de vano de la línea de carga, lugar donde se experimentan las máximos valores de desplazamiento. Son un total de 3 puntos, cuya localización puede apreciarse en la figura 4.9 . 


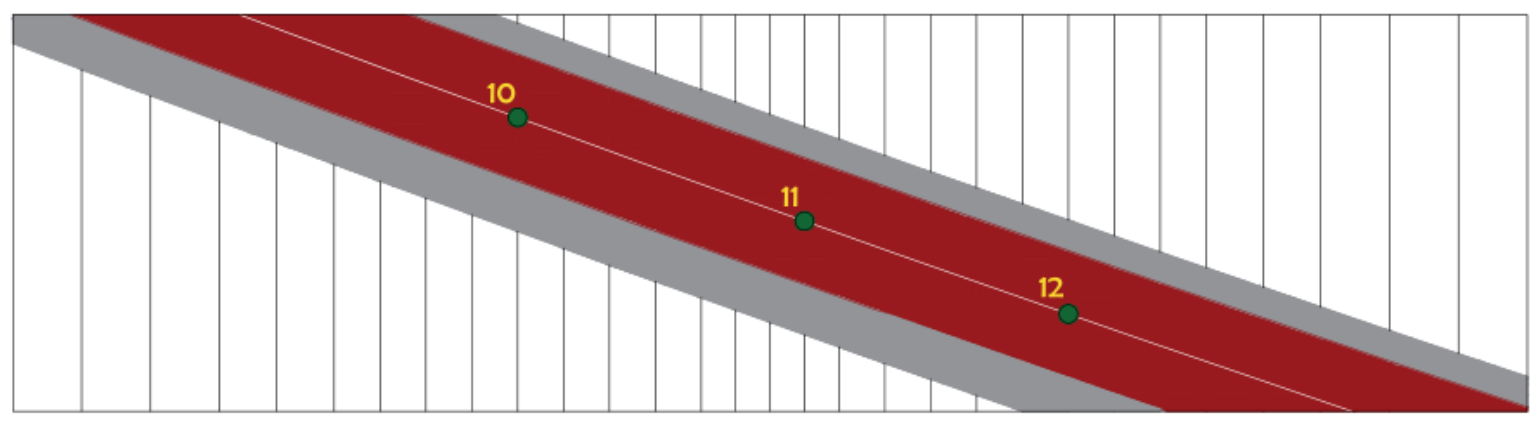

Figura 4.9: Puntos de posproceso de valores de desplazamiento. Pérgola de Bobadilla II

\subsubsection{Datos de partida}

Los datos necesarios para el cálculo de la rigidez y amortiguamiento en los pilotes de la pérgola de Bobadilla II son los siguientes:

- $E_{p}: 3 \cdot 10^{10} \mathrm{~N} / \mathrm{m}^{2}$

- $V_{s}: 250 \mathrm{~m} / \mathrm{s}$

- R: $0.6 \mathrm{~m}$

- $1: 30 \mathrm{~m}$

- A: $1.131 \mathrm{~m}^{2}$

- I: $0.101787 m^{4}$

A partir de los datos anteriores se procede a obtener la relación existente entre la longitud y el radio del pilote.

$$
\frac{l}{R}=\frac{30}{0.6}=50
$$

Una vez se ha obtenido la relación entre la longitud y el radio del pilote se deben obtener los valores de $f_{v 1}$ y $f_{v 2}$ para la tipología de pilote a la que pertenezca. Los pilotes de la pérgola de Bobadilla II y el terreno existente a su alrededor tienen las siguientes características:

- Pilote flotante

- Rigidez del terreno ascendente de forma parabólica en el sentido vertical descendente

En la figura 4.10 se pueden observar los valores de $f_{v 1}$ y $f_{v 2}$ para la esbeltez calculada mediante la fórmula (4.5). 


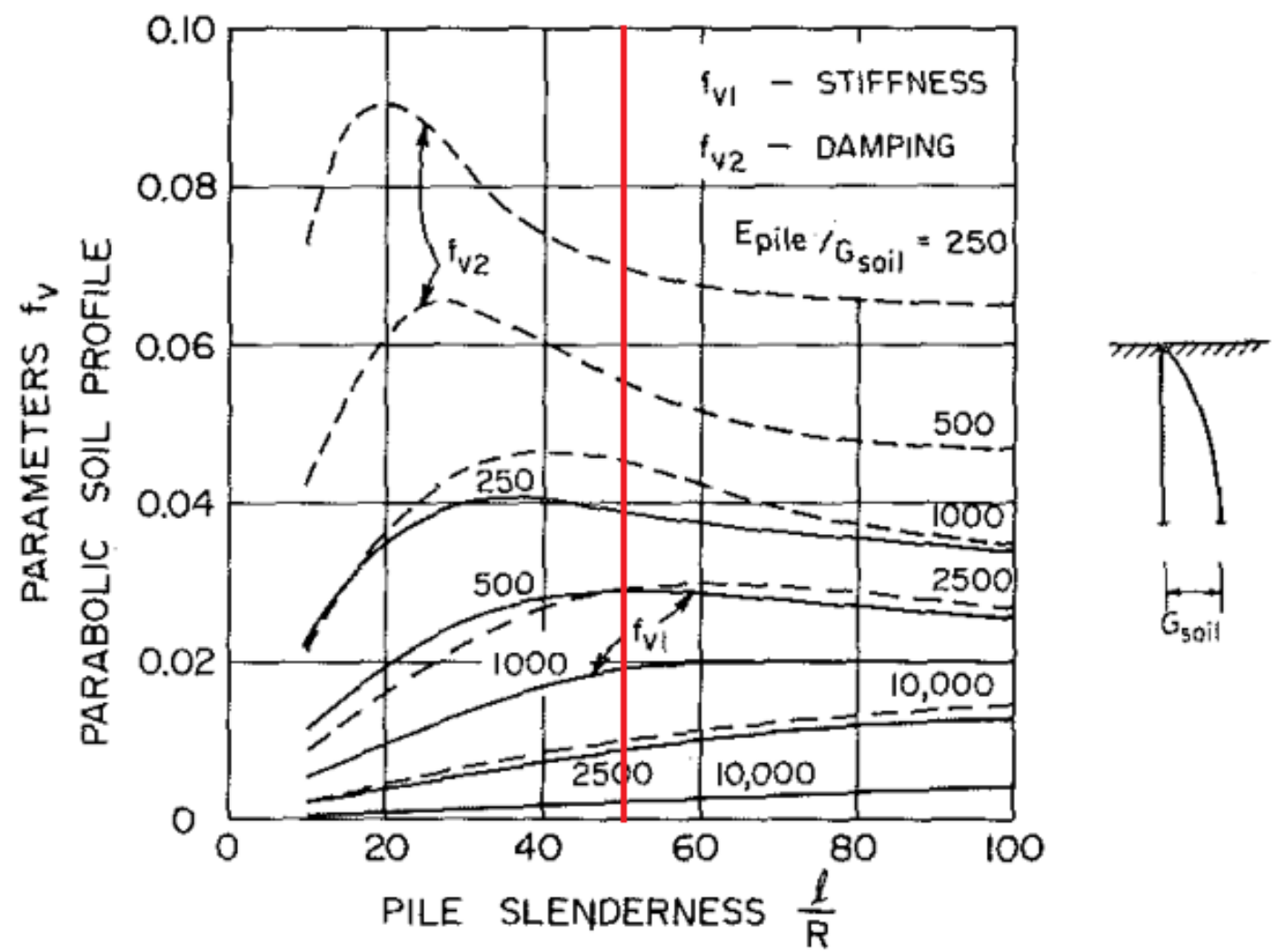

Figura 4.10: Valores de $f_{v 1}$ y $f_{v 2}$ para $\frac{l}{R}=50$. Novak et al. [28]

Una vez obtenidos los valores de $f_{v 1}$ y $f_{v 2}$ para todas las relaciones de rigidez $\left(E_{p} / G_{s}\right)$ presentes en la gráfica 4.10 se procede a elaborar una curva de tendencia para cada parámetro, con el fin de poder realizar el análisis para relaciones de rigidez distintas a las utilizadas por Novak et al. [28].

La línea de tendencia para el valor $f_{v 1}$ se puede observar en la figura 4.11. Su ecuación es la (4.6). 


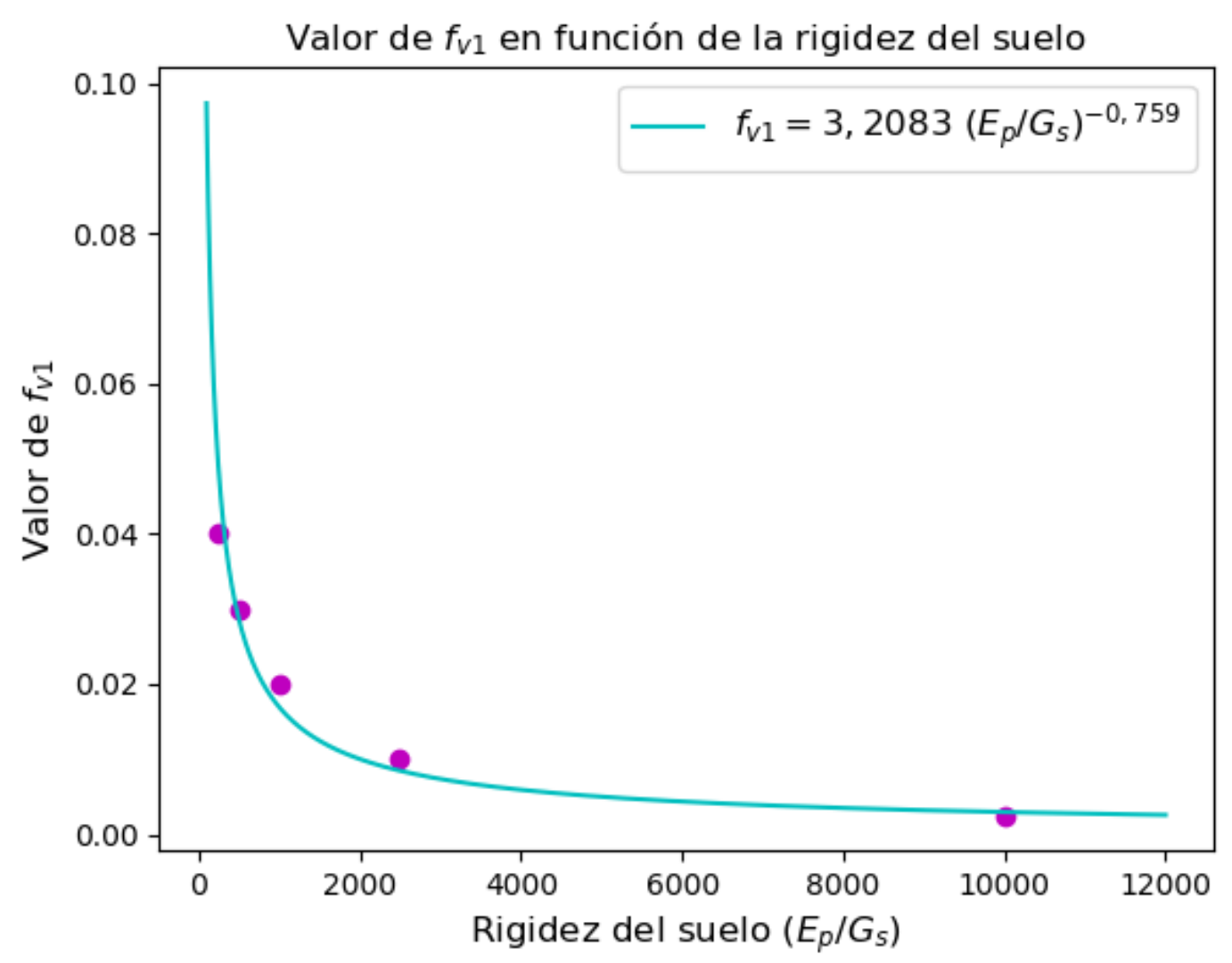

Figura 4.11: Valores de $f_{v 1}$. Pérgola de Bobadilla II

$$
f_{v 1}=3.2083\left(\frac{E_{p}}{G_{s}}\right)^{-0.759} \quad R^{2}=0.9749
$$

Los valores de $f_{v 1}$ para los casos de estudio se pueden observar en la tabla 4.1.

\begin{tabular}{|c|c|}
\hline$E_{p} / G_{s}$ & $f_{v 1}$ \\
\hline 250 & 0.04 \\
\hline 500 & 0.03 \\
\hline 1000 & 0.02 \\
\hline 2500 & 0.01 \\
\hline 5000 & 0.005 \\
\hline 10000 & 0.0025 \\
\hline
\end{tabular}

Cuadro 4.1: Valores de $f_{v 1}$ para distintos casos de rigidez. Pérgola de Bobadilla II

La línea de tendencia para el valor $f_{v 2}$ se puede observar en la figura 4.12. Su ecuación es la (4.7). 


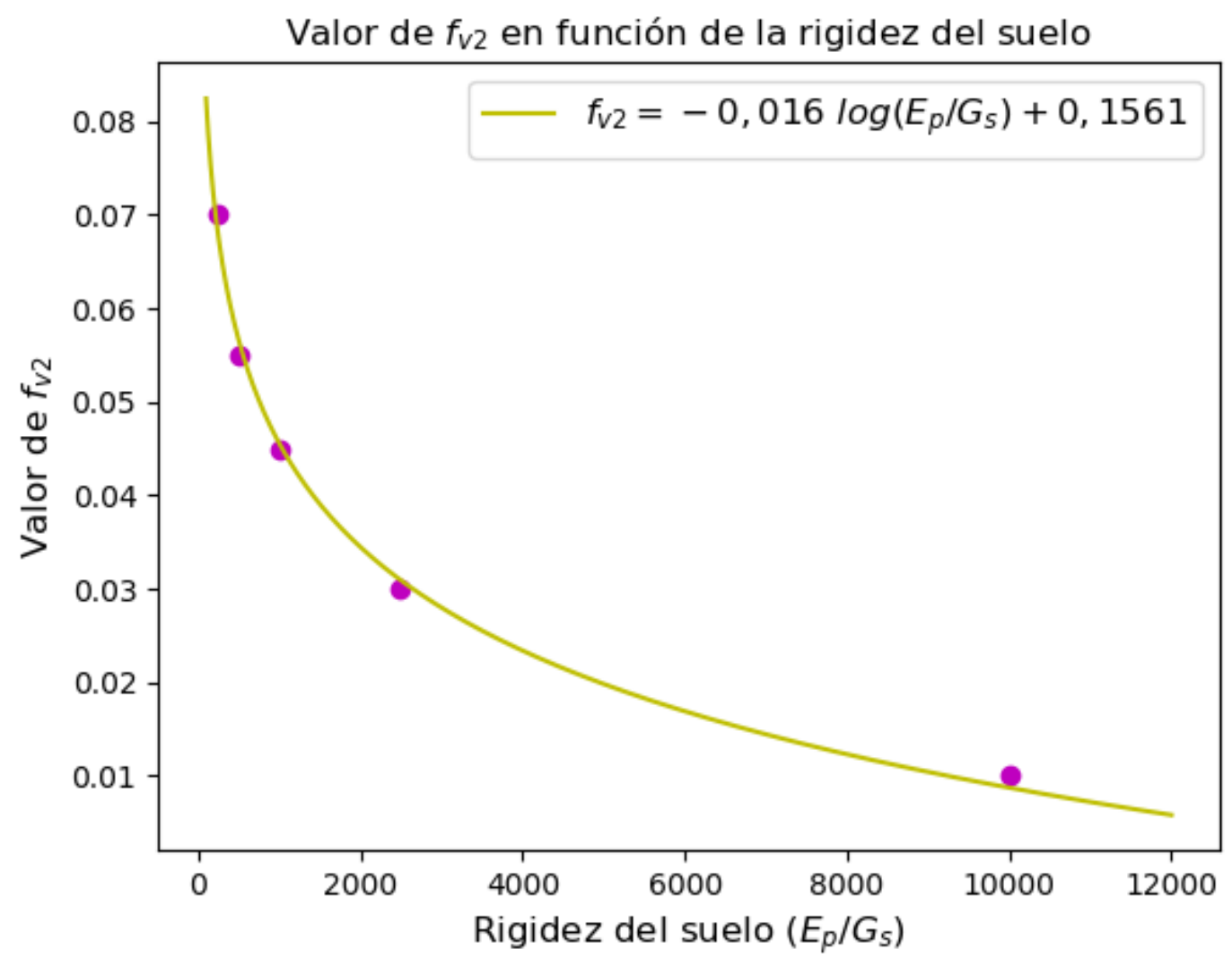

Figura 4.12: Valores de $f_{v 2}$. Pérgola de Bobadilla II

$$
f_{v 2}=-0.016 \log \left(\frac{E_{p}}{G_{s}}\right)+0.1561 \quad R^{2}=0.995
$$

Los valores de $f_{v 2}$ para los casos de estudio se pueden observar en la tabla 4.2.

\begin{tabular}{|c|c|}
\hline$E_{p} / G_{s}$ & $f_{v 2}$ \\
\hline 250 & 0.07 \\
\hline 500 & 0.055 \\
\hline 1000 & 0.045 \\
\hline 2500 & 0.03 \\
\hline 5000 & 0.02 \\
\hline 10000 & 0.01 \\
\hline
\end{tabular}

Cuadro 4.2: Valores de $f_{v 2}$ para distintos casos de rigidez. Pérgola de Bobadilla II

A partir de la ecuación (3.7) y la línea de tendencia obtenida para $f_{v 1}$ de la figura 4.11, cuya ecuación es la (4.6), es posible expresar la rigidez vertical $\left(k_{v}\right)$ de forma analítica.

La gráfica que representa $\left(k_{v}\right)$ es la figura 4.13 . 


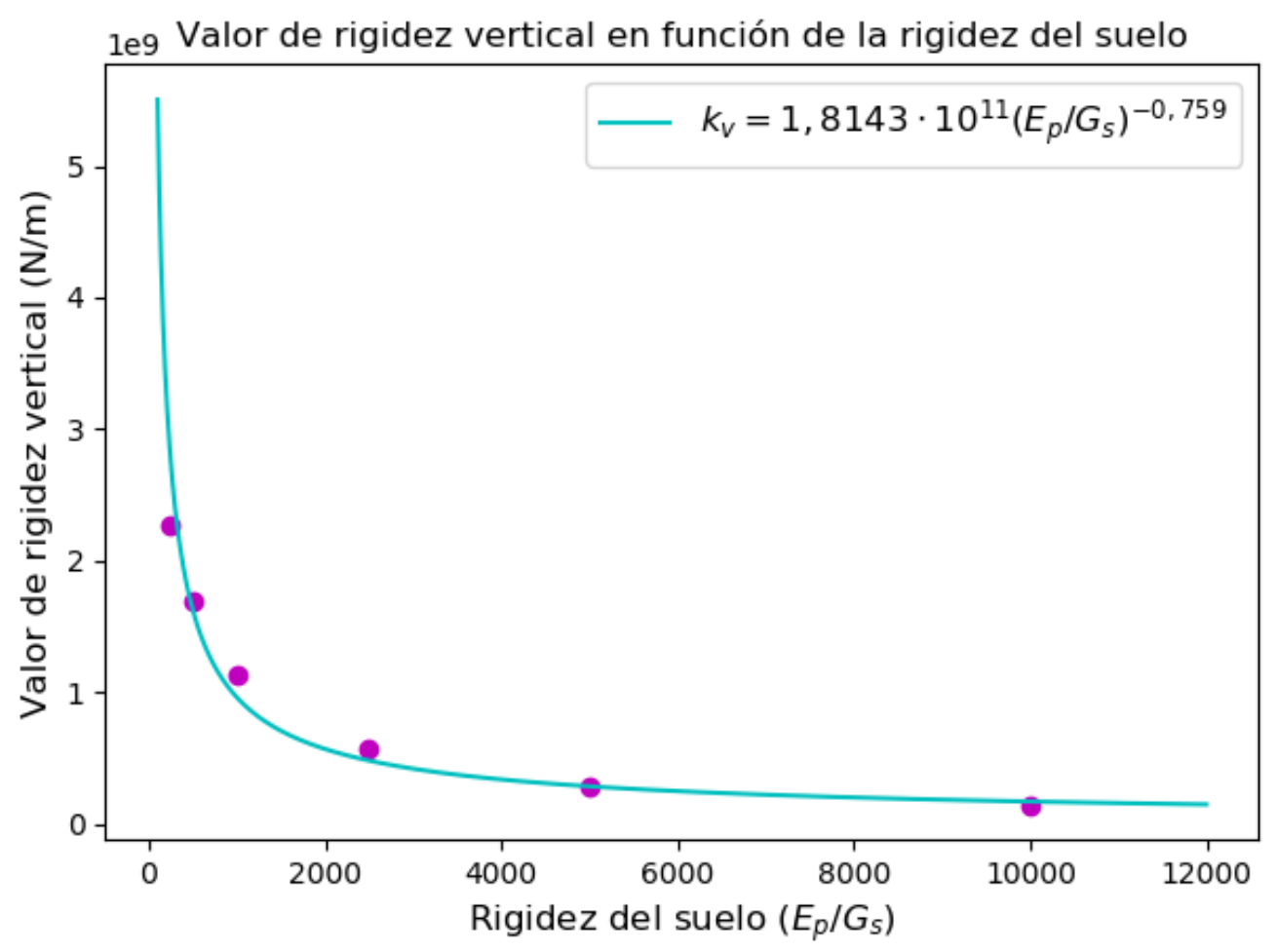

Figura 4.13: Valores de $k_{v}$. Pérgola de Bobadilla II

Y su ecuación es la (4.8).

$$
k_{v}=1.8143 \cdot 10^{11}\left(\frac{E_{p}}{G_{s}}\right)^{-0.759}
$$

De manera análoga se puede calcular una expresión análitica para el amortiguamiento vertical $\left(c_{v}\right)$. En esta ocasión se utiliza la línea de tendencia de $f_{v 2}$ (ecuación (4.7)).

La gráfica que representa $\left(c_{v}\right)$ es la figura 4.14 . 


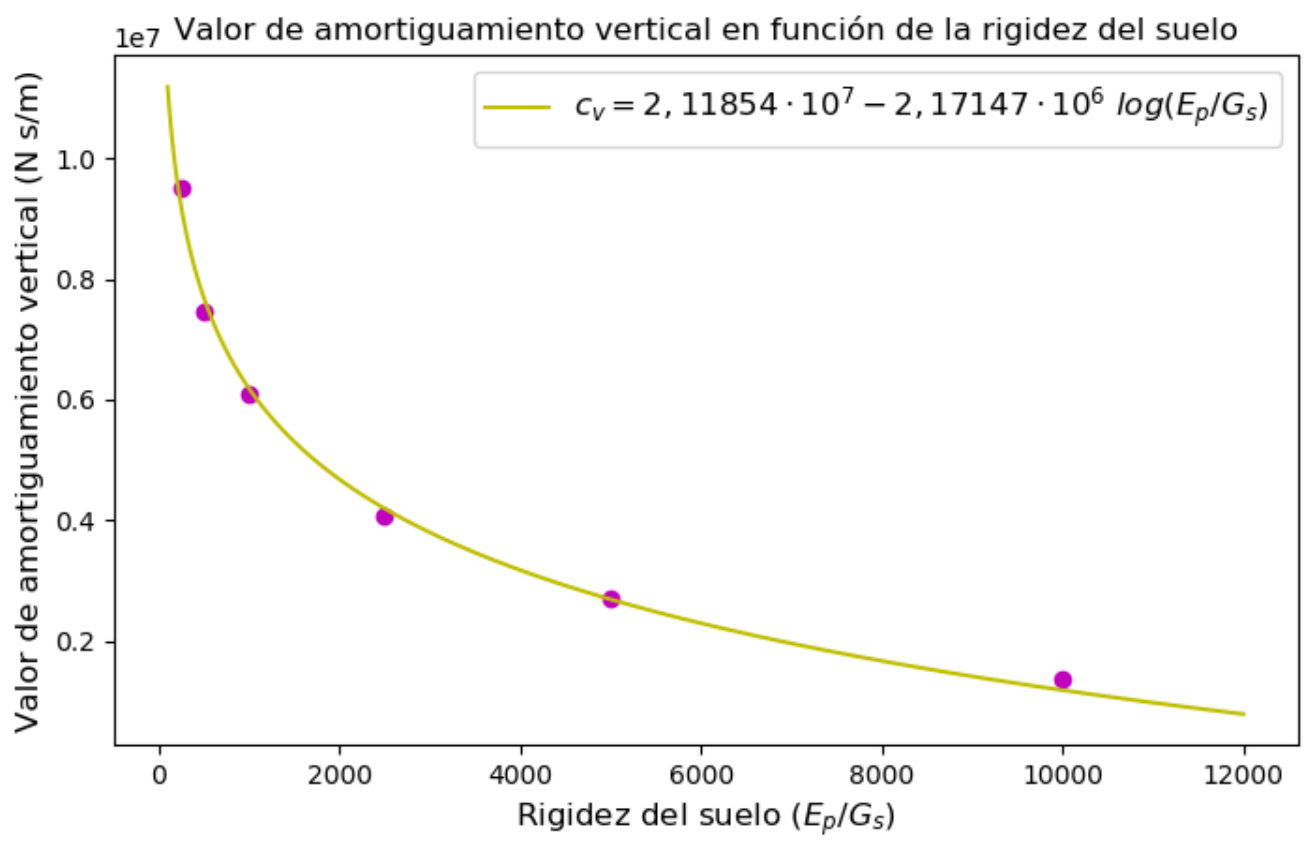

Figura 4.14: Valores de $k_{v}$. Pérgola de Bobadilla II

Y su ecuación es la (4.9).

$$
c_{v}=2.11854 \cdot 10^{7}-2.17147 \cdot 10^{6} \log \left(\frac{E_{p}}{G_{s}}\right)
$$

Los valores de $k_{v}$ y $c_{v}$ para cada los distintos casos de rigidez del suelo contemplados en este trabajo pueden observarse en la tabla 4.3.

\begin{tabular}{|c|c|c|}
\hline$E_{p} / G_{s}$ & $k_{v}(\mathrm{~N} / m)$ & $c_{v}\left(\frac{\mathrm{NS}}{m}\right)$ \\
\hline 250 & 2261946710.58 & 9500176.18 \\
\hline 500 & 1696460032.93 & 7464424.14 \\
\hline 1000 & 1130973355.29 & 6107256.11 \\
\hline 2500 & 565486677.64 & 4071504.07 \\
\hline 5000 & 282743338.82 & 2714336.05 \\
\hline 10000 & 141371669.41 & 1357168.02 \\
\hline
\end{tabular}

Cuadro 4.3: Valores de $k_{v}$ y $c_{v}$ para distintos casos de rigidez. Pérgola de Bobadilla II

\subsubsection{Cálculo dinámico}

Para el cálculo dinámico se van a seguir las siguientes directrices.

- Modos de vibración con frecuencia inferior a $30 \mathrm{~Hz}$

- Amortiguamiento estructural constante igual al $2 \%$

- Amortiguamiento por la interacción suelo-estructura propio para cada modo calculado por SAP2000 
Estructura con apoyos rígidos

Estructura con apoyos. Análisis modal

Los modos de vibración con frecuencia inferior a $30 \mathrm{~Hz}$ se pueden observar en la tabla 4.4. En este caso no existe amortiguamiento por interacción suelo-estructura.

\begin{tabular}{|c|c|c|}
\hline Modo & Período (s) & Frecuencia $(\mathrm{Hz})$ \\
\hline 1 & 0.1560 & 6.4094 \\
\hline 2 & 0.1520 & 6.5811 \\
\hline 3 & 0.1469 & 6.8089 \\
\hline 4 & 0.1419 & 7.0469 \\
\hline 5 & 0.1397 & 7.1558 \\
\hline 6 & 0.1395 & 7.1694 \\
\hline 7 & 0.1362 & 7.3426 \\
\hline 8 & 0.1309 & 7.6402 \\
\hline 9 & 0.1266 & 7.8965 \\
\hline 10 & 0.1250 & 7.9994 \\
\hline 11 & 0.1240 & 8.0673 \\
\hline 12 & 0.1213 & 8.2474 \\
\hline 13 & 0.1201 & 8.3294 \\
\hline 14 & 0.1148 & 8.7124 \\
\hline 15 & 0.1136 & 8.8005 \\
\hline 16 & 0.1100 & 9.0912 \\
\hline 17 & 0.1090 & 9.1779 \\
\hline 18 & 0.1059 & 9.4386 \\
\hline 19 & 0.1030 & 9.7072 \\
\hline 20 & 0.0999 & 10.0120 \\
\hline 21 & 0.0973 & 10.2790 \\
\hline 22 & 0.0950 & 10.5310 \\
\hline 23 & 0.0929 & 10.7620 \\
\hline 24 & 0.0893 & 11.2010 \\
\hline 25 & 0.0889 & 11.2520 \\
\hline 26 & 0.0862 & 11.6060 \\
\hline 27 & 0.0849 & 11.7840 \\
\hline 28 & 0.0821 & 12.1810 \\
\hline 29 & 0.0813 & 12.3060 \\
\hline 30 & 0.0782 & 12.7830 \\
\hline 31 & 0.0762 & 13.1300 \\
\hline 32 & 0.0734 & 13.6180 \\
\hline
\end{tabular}

Continúa en la página siguiente. 


\begin{tabular}{|c|c|c|}
\hline Modo & Período (s) & Frecuencia $(\mathrm{Hz})$ \\
\hline 33 & 0.0712 & 14.0360 \\
\hline 34 & 0.0709 & 14.1030 \\
\hline 35 & 0.0693 & 14.4370 \\
\hline 36 & 0.0671 & 14.9030 \\
\hline 37 & 0.0666 & 15.0260 \\
\hline 38 & 0.0655 & 15.2680 \\
\hline 39 & 0.0637 & 15.6960 \\
\hline 40 & 0.0630 & 15.8830 \\
\hline 41 & 0.0612 & 16.3430 \\
\hline 42 & 0.0594 & 16.8340 \\
\hline 43 & 0.0585 & 17.0890 \\
\hline 44 & 0.0568 & 17.6000 \\
\hline 45 & 0.0563 & 17.7520 \\
\hline 46 & 0.0559 & 17.8890 \\
\hline 47 & 0.0542 & 18.4650 \\
\hline 48 & 0.0527 & 18.9930 \\
\hline 49 & 0.0513 & 19.4990 \\
\hline 50 & 0.0510 & 19.5930 \\
\hline 51 & 0.0506 & 19.7610 \\
\hline 52 & 0.0502 & 19.9150 \\
\hline 53 & 0.0471 & 21.2170 \\
\hline 54 & 0.0466 & 21.4410 \\
\hline 55 & 0.0461 & 21.6910 \\
\hline 56 & 0.0452 & 22.1030 \\
\hline 57 & 0.0443 & 22.5690 \\
\hline 58 & 0.0433 & 23.1090 \\
\hline 59 & 0.0432 & 23.1520 \\
\hline 60 & 0.0427 & 23.4140 \\
\hline 61 & 0.0413 & 24.1850 \\
\hline 62 & 0.0404 & 24.7750 \\
\hline 63 & 0.0401 & 24.9330 \\
\hline 64 & 0.0399 & 25.0390 \\
\hline 65 & 0.0391 & 25.5700 \\
\hline 66 & 0.0388 & 25.7550 \\
\hline 67 & 0.0385 & 26.0010 \\
\hline 68 & 0.0377 & 26.5580 \\
\hline 69 & 0.0375 & 26.6690 \\
\hline
\end{tabular}

Continúa en la página siguiente. 


\begin{tabular}{|c|c|c|}
\hline Modo & Período (s) & Frecuencia $(\mathrm{Hz})$ \\
\hline \hline 70 & 0.0374 & 26.7350 \\
\hline 71 & 0.0369 & 27.0800 \\
\hline 72 & 0.0363 & 27.5380 \\
\hline 73 & 0.0362 & 27.6500 \\
\hline 74 & 0.0360 & 27.7980 \\
\hline 75 & 0.0358 & 27.9710 \\
\hline 76 & 0.0356 & 28.1150 \\
\hline 77 & 0.0352 & 28.3780 \\
\hline 78 & 0.0350 & 28.5360 \\
\hline 79 & 0.0348 & 28.7040 \\
\hline 80 & 0.0348 & 28.7490 \\
\hline 81 & 0.0347 & 28.8210 \\
\hline 82 & 0.0346 & 28.8660 \\
\hline 83 & 0.0345 & 28.9670 \\
\hline 84 & 0.0343 & 29.1350 \\
\hline 85 & 0.0341 & 29.3340 \\
\hline 86 & 0.0338 & 29.5490 \\
\hline 87 & 0.0336 & 29.7220 \\
\hline 88 & 0.0335 & 29.8180 \\
\hline
\end{tabular}

Cuadro 4.4: Valores de período y frecuencia para estructura con apoyos. Pérgola de Bobadilla II

La forma de los tres primeros modos de vibración se puede apreciar en las figuras 4.15 , 4.16 y 4.16 . 


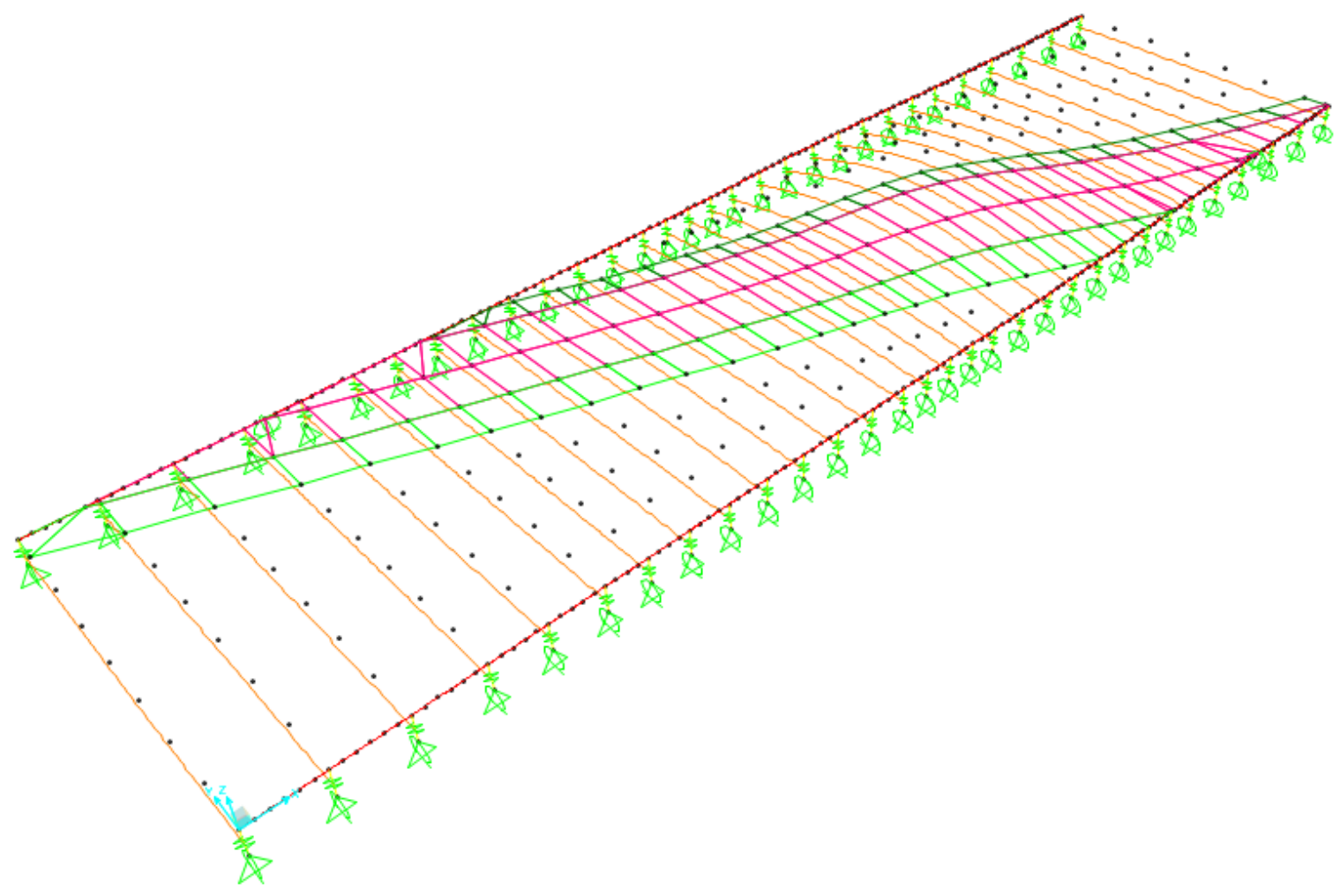

Figura 4.15: Modo 1 de vibración. Pérgola de Bobadilla II

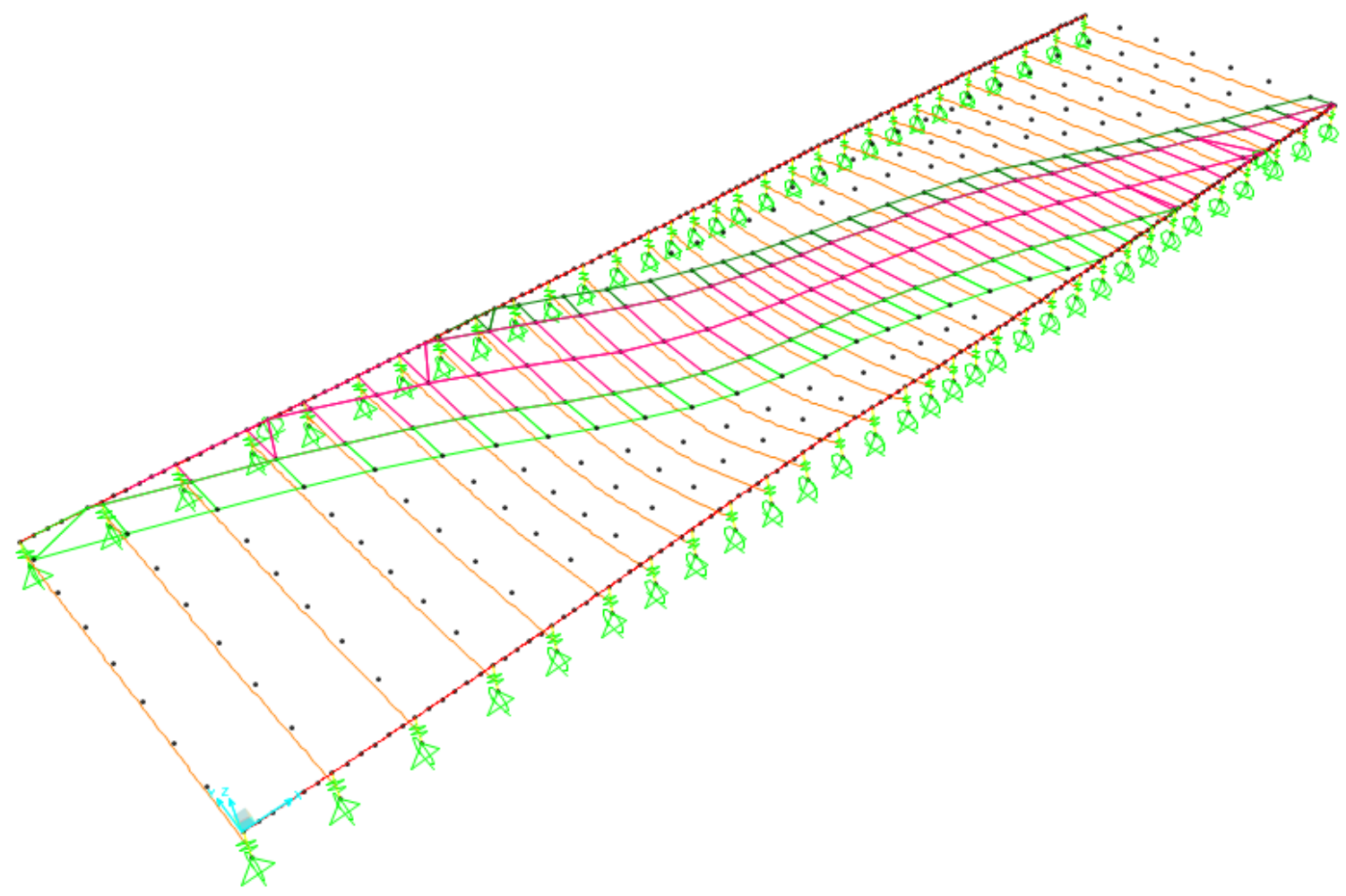

Figura 4.16: Modo 2 de vibración. Pérgola de Bobadilla II 


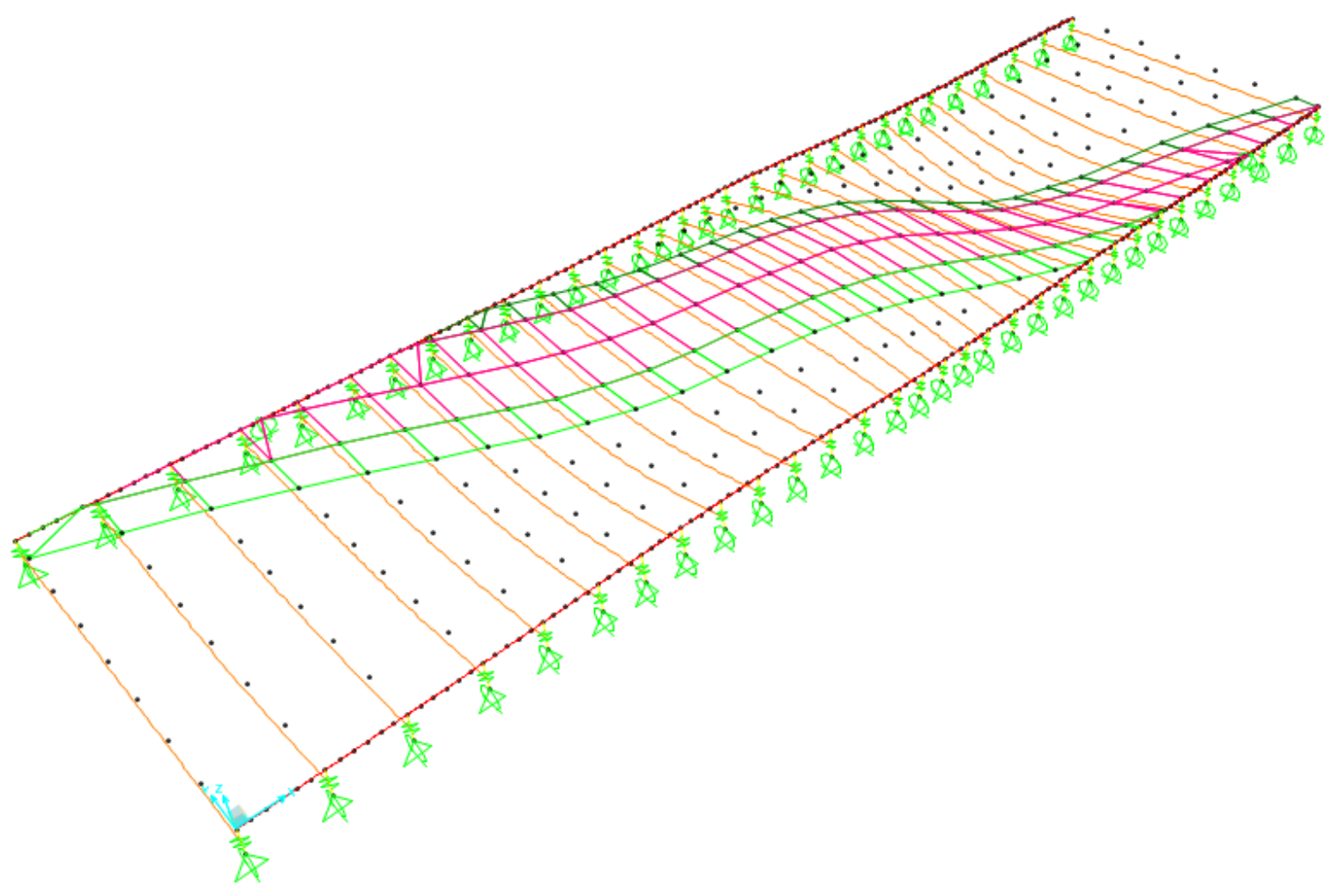

Figura 4.17: Modo 3 de vibración. Pérgola de Bobadilla II

Estructura con apoyos. Resultados de aceleración

En la tabla 4.5 se puede observar toda la información relativa al punto de aceleración máxima.

\begin{tabular}{|c|c|}
\hline Máximo valor de aceleración & $4.320 \mathrm{~m} / \mathrm{s}^{2}$ \\
\hline Punto & 2 \\
\hline Tren & 2 \\
\hline Velocidad & $240 \mathrm{~km} / \mathrm{h}$ \\
\hline
\end{tabular}

Cuadro 4.5: Máxima aceleración para estrutura con apoyos. Pérgola de Bobadilla II

En la figura 4.18 se puede observar la envolvente de aceleración en el punto en el que se alcanza su valor máximo. 


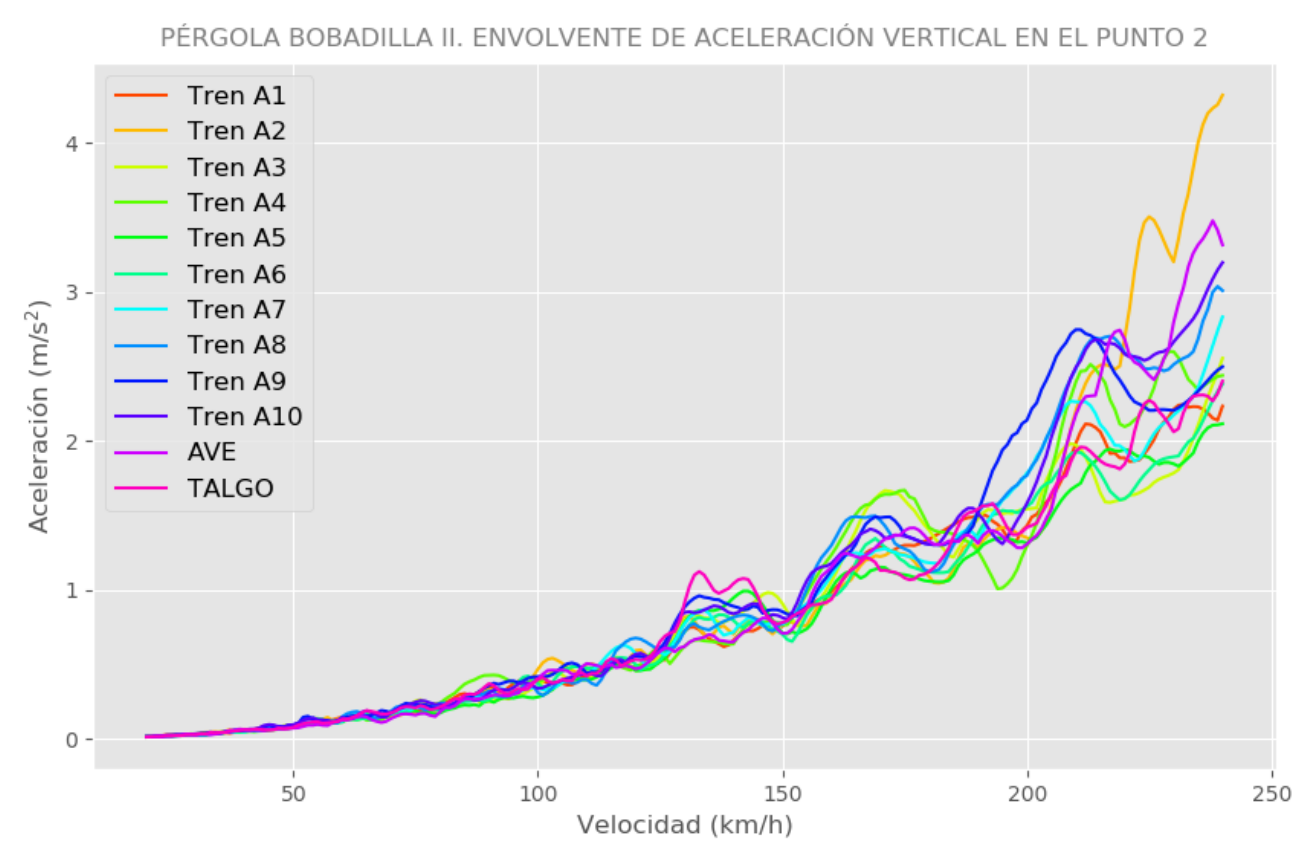

Figura 4.18: Envolvente de aceleración en el punto 2 en estructura con apoyos. Pérgola de Bobadilla II

Estructura con apoyos. Resultados de desplazamiento

En la tabla 4.6 se puede observar toda la información relativa al punto de aceleración máxima.

\begin{tabular}{|c|c|}
\hline Máximo valor de desplazamiento & $2.717 \mathrm{~mm}$ \\
\hline Punto & 11 \\
\hline Tren & 10 \\
\hline Velocidad & $240 \mathrm{~km} / \mathrm{h}$ \\
\hline
\end{tabular}

Cuadro 4.6: Máximo desplazamiento para estrutura con apoyos. Pérgola de Bobadilla II

En la figura 4.19 se puede observar la envolvente de desplazamiento en el punto en el que se alcanza su valor máximo. 


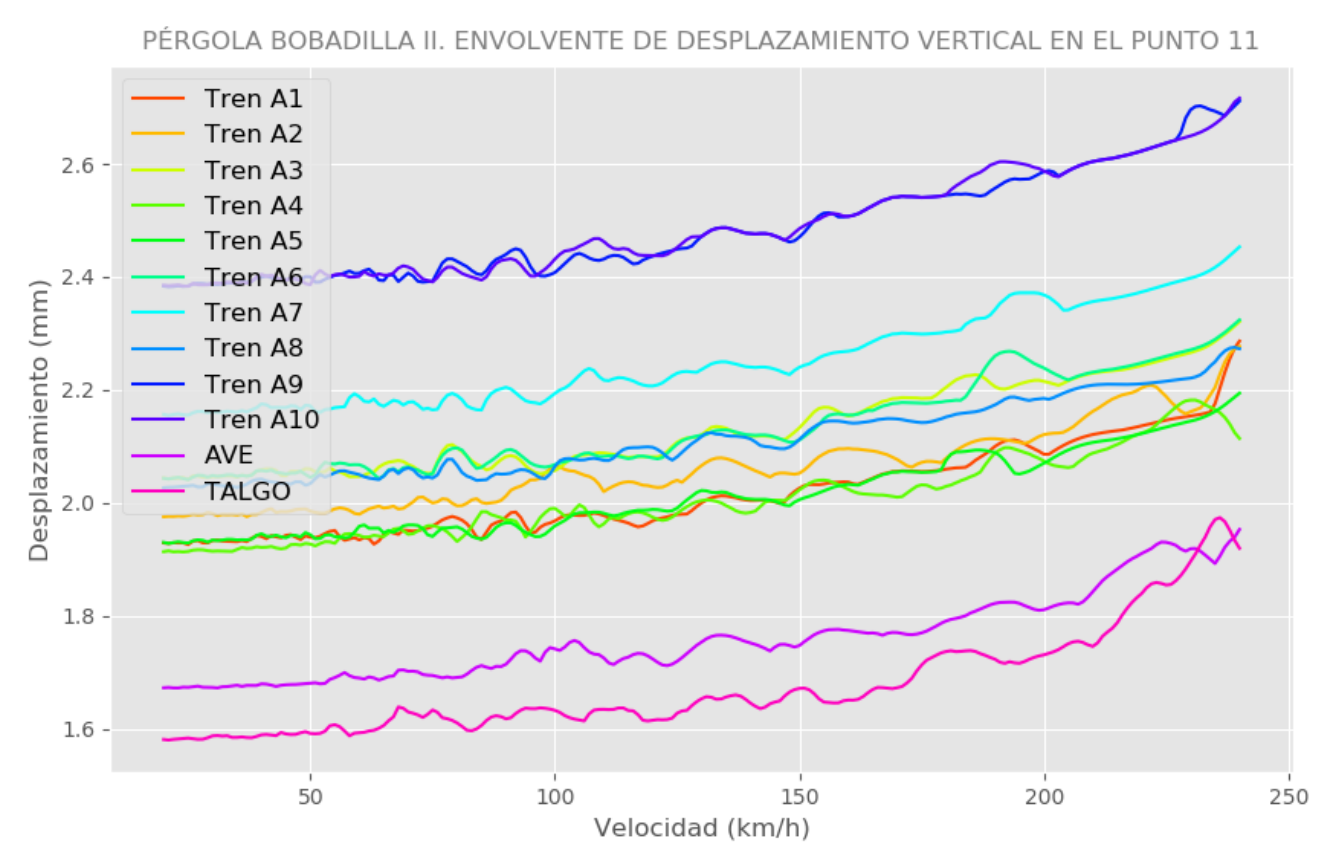

Figura 4.19: Envolvente de desplazamiento en el punto 11 en estructura con apoyos. Pérgola de Bobadilla II

Estructura con $E_{p} / G_{s}=250$

$E_{p} / G_{s}=250$. Análisis modal

Los modos de vibración con frecuencia inferior a $30 \mathrm{~Hz}$ y su amortiguamiento por la interacción suelo estructura se pueden observar en la tabla 4.7.

\begin{tabular}{|c|c|c|c|}
\hline Modo & Período (s) & Frecuencia $(\mathrm{Hz})$ & Amortiguamiento por interacción suelo - estructura \\
\hline \hline 1 & 0.1572 & 6.3626 & 0.001276 \\
\hline 2 & 0.1531 & 6.5302 & 0.001390 \\
\hline 3 & 0.1479 & 6.7609 & 0.001315 \\
\hline 4 & 0.1428 & 7.0005 & 0.001274 \\
\hline 5 & 0.1398 & 7.1516 & 0.000139 \\
\hline 6 & 0.1395 & 7.1668 & 0.000061 \\
\hline 7 & 0.1370 & 7.2996 & 0.001138 \\
\hline 8 & 0.1316 & 7.5965 & 0.001167 \\
\hline 9 & 0.1273 & 7.8579 & 0.001056 \\
\hline 10 & 0.1253 & 7.9834 & 0.000488 \\
\hline 11 & 0.1242 & 8.0546 & 0.000373 \\
\hline 12 & 0.1216 & 8.2220 & 0.000669 \\
\hline 13 & 0.1204 & 8.3077 & 0.000581 \\
\hline
\end{tabular}

Continúa en la página siguiente. 


\begin{tabular}{|c|c|c|c|}
\hline Modo & Período (s) & Frecuencia $(\mathrm{Hz})$ & Amortiguamiento por interacción suelo - estructura \\
\hline 14 & 0.1153 & 8.6758 & 0.001013 \\
\hline 15 & 0.1139 & 8.7808 & 0.000551 \\
\hline 16 & 0.1104 & 9.0592 & 0.000916 \\
\hline 17 & 0.1092 & 9.1610 & 0.000473 \\
\hline 18 & 0.1061 & 9.4239 & 0.000408 \\
\hline 19 & 0.1033 & 9.6775 & 0.000824 \\
\hline 20 & 0.1002 & 9.9767 & 0.001006 \\
\hline 21 & 0.0974 & 10.2640 & 0.000426 \\
\hline 22 & 0.0951 & 10.5100 & 0.000620 \\
\hline 23 & 0.0931 & 10.7420 & 0.000525 \\
\hline 24 & 0.0895 & 11.1730 & 0.000975 \\
\hline 25 & 0.0891 & 11.2210 & 0.000745 \\
\hline 26 & 0.0864 & 11.5710 & 0.001072 \\
\hline 27 & 0.0850 & 11.7630 & 0.000539 \\
\hline 28 & 0.0823 & 12.1520 & 0.000948 \\
\hline 29 & 0.0814 & 12.2790 & 0.000701 \\
\hline 30 & 0.0783 & 12.7710 & 0.000372 \\
\hline 31 & 0.0764 & 13.0910 & 0.001191 \\
\hline 32 & 0.0735 & 13.6080 & 0.000308 \\
\hline 33 & 0.0714 & 14.0000 & 0.001199 \\
\hline 34 & 0.0710 & 14.0900 & 0.000371 \\
\hline 35 & 0.0694 & 14.4150 & 0.000645 \\
\hline 36 & 0.0672 & 14.8740 & 0.001047 \\
\hline 37 & 0.0667 & 15.0040 & 0.000674 \\
\hline 38 & 0.0656 & 15.2490 & 0.000553 \\
\hline 39 & 0.0638 & 15.6860 & 0.000335 \\
\hline 40 & 0.0630 & 15.8730 & 0.000360 \\
\hline 41 & 0.0614 & 16.2900 & 0.001793 \\
\hline 42 & 0.0594 & 16.8240 & 0.000340 \\
\hline 43 & 0.0585 & 17.0810 & 0.000294 \\
\hline 44 & 0.0569 & 17.5600 & 0.001623 \\
\hline 45 & 0.0564 & 17.7310 & 0.000677 \\
\hline 46 & 0.0559 & 17.8760 & 0.000405 \\
\hline 47 & 0.0542 & 18.4540 & 0.000460 \\
\hline 48 & 0.0527 & 18.9870 & 0.000272 \\
\hline 49 & 0.0515 & 19.4360 & 0.002765 \\
\hline 50 & 0.0511 & 19.5760 & 0.000498 \\
\hline
\end{tabular}

Continúa en la página siguiente. 


\begin{tabular}{|c|c|c|c|}
\hline Modo & Período (s) & Frecuencia $(\mathrm{Hz})$ & Amortiguamiento por interacción suelo - estructura \\
\hline 51 & 0.0506 & 19.7550 & 0.000207 \\
\hline 52 & 0.0502 & 19.9060 & 0.000340 \\
\hline 53 & 0.0474 & 21.1110 & 0.004777 \\
\hline 54 & 0.0467 & 21.4290 & 0.000597 \\
\hline 55 & 0.0461 & 21.6780 & 0.000647 \\
\hline 56 & 0.0453 & 22.0850 & 0.001104 \\
\hline 57 & 0.0443 & 22.5580 & 0.000632 \\
\hline 58 & 0.0435 & 22.9710 & 0.007824 \\
\hline 59 & 0.0433 & 23.0880 & 0.003755 \\
\hline 60 & 0.0428 & 23.3730 & 0.002908 \\
\hline 61 & 0.0414 & 24.1470 & 0.003648 \\
\hline 62 & 0.0405 & 24.6880 & 0.011204 \\
\hline 63 & 0.0404 & 24.7340 & 0.013059 \\
\hline 64 & 0.0401 & 24.9110 & 0.025098 \\
\hline 65 & 0.0398 & 25.1510 & 0.072679 \\
\hline 66 & 0.0393 & 25.4310 & 0.098788 \\
\hline 67 & 0.0391 & 25.5570 & 0.030694 \\
\hline 68 & 0.0391 & 25.6050 & 0.115136 \\
\hline 69 & 0.0389 & 25.6850 & 0.032970 \\
\hline 70 & 0.0387 & 25.8400 & 0.095243 \\
\hline 71 & 0.0386 & 25.9130 & 0.090607 \\
\hline 72 & 0.0385 & 25.9760 & 0.145698 \\
\hline 73 & 0.0382 & 26.1540 & 0.170270 \\
\hline 74 & 0.0382 & 26.1930 & 0.095211 \\
\hline 75 & 0.0379 & 26.3640 & 0.025744 \\
\hline 76 & 0.0379 & 26.3900 & 0.159608 \\
\hline 77 & 0.0378 & 26.4500 & 0.165070 \\
\hline 78 & 0.0375 & 26.6590 & 0.155826 \\
\hline 79 & 0.0374 & 26.7580 & 0.041543 \\
\hline 80 & 0.0371 & 26.9730 & 0.150327 \\
\hline 81 & 0.0369 & 27.0760 & 0.158579 \\
\hline 82 & 0.0366 & 27.3070 & 0.163081 \\
\hline 83 & 0.0363 & 27.5490 & 0.098601 \\
\hline 84 & 0.0362 & 27.6050 & 0.104007 \\
\hline 85 & 0.0361 & 27.7320 & 0.152585 \\
\hline 86 & 0.0359 & 27.8250 & 0.127430 \\
\hline 87 & 0.0357 & 27.9950 & 0.188080 \\
\hline
\end{tabular}

Continúa en la página siguiente. 


\begin{tabular}{|c|c|c|c|}
\hline Modo & Período (s) & Frecuencia $(\mathrm{Hz})$ & Amortiguamiento por interacción suelo - estructura \\
\hline \hline 88 & 0.0355 & 28.1390 & 0.077048 \\
\hline 89 & 0.0354 & 28.2670 & 0.121093 \\
\hline 90 & 0.0353 & 28.3350 & 0.064141 \\
\hline 91 & 0.0352 & 28.3710 & 0.105000 \\
\hline 92 & 0.0351 & 28.4690 & 0.164873 \\
\hline 93 & 0.0349 & 28.6350 & 0.186433 \\
\hline 94 & 0.0348 & 28.7160 & 0.031108 \\
\hline 95 & 0.0346 & 28.9110 & 0.070686 \\
\hline 96 & 0.0344 & 29.0740 & 0.068305 \\
\hline 97 & 0.0342 & 29.2250 & 0.228225 \\
\hline 98 & 0.0341 & 29.3320 & 0.210991 \\
\hline 99 & 0.0337 & 29.6320 & 0.251851 \\
\hline 100 & 0.0336 & 29.7290 & 0.108680 \\
\hline 101 & 0.0335 & 29.8420 & 0.353863 \\
\hline 102 & 0.0334 & 29.9330 & 0.366850 \\
\hline 103 & 0.0334 & 29.9480 & 0.344265 \\
\hline
\end{tabular}

Cuadro 4.7: Valores de período, frecuencia y amortiguamiento para $E_{p} / G_{s}=250$. Pérgola de Bobadilla II

$E_{p} / G_{s}=250$. Resultados de aceleración

En la tabla 4.8 se puede observar toda la información relativa al punto de aceleración máxima.

\begin{tabular}{|c|c|}
\hline Máximo valor de aceleración & $3.828 \mathrm{~m} / \mathrm{s}^{2}$ \\
\hline Punto & 2 \\
\hline Tren & 2 \\
\hline Velocidad & $238 \mathrm{~km} / \mathrm{h}$ \\
\hline
\end{tabular}

Cuadro 4.8: Máxima aceleración para $E_{p} / G_{s}=250$. Pérgola de Bobadilla II

En la figura 4.20 se puede observar la envolvente de aceleración en el punto en el que se alcanza su valor máximo. 


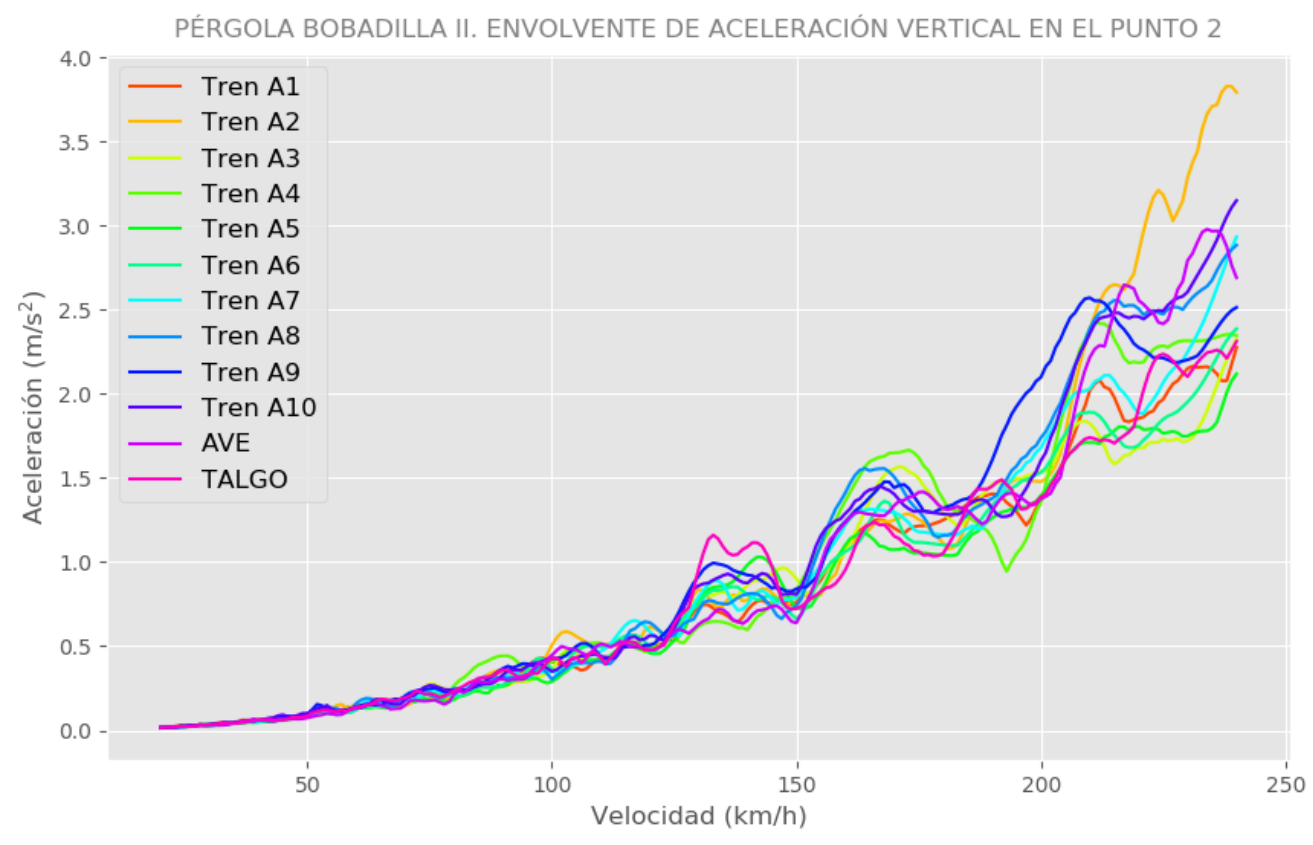

Figura 4.20: Envolvente de aceleración en el punto 2 para $E_{p} / G_{s}=250$. Pérgola de Bobadilla II

$$
E_{p} / G_{s}=250 . \text { Resultados de desplazamiento }
$$

En la tabla 4.9 se puede observar toda la información relativa al punto de desplazamiento máximo.

\begin{tabular}{|c|c|}
\hline Máximo valor de desplazamiento & $2.753 \mathrm{~mm}$ \\
\hline Punto & 11 \\
\hline Tren & 10 \\
\hline Velocidad & $240 \mathrm{~km} / \mathrm{h}$ \\
\hline
\end{tabular}

Cuadro 4.9: Máximo desplazamiento para $E_{p} / G_{s}=250$. Pérgola de Bobadilla II

En la figura 4.21 se puede observar la envolvente de desplazamiento en el punto en el que se alcanza su valor máximo. 


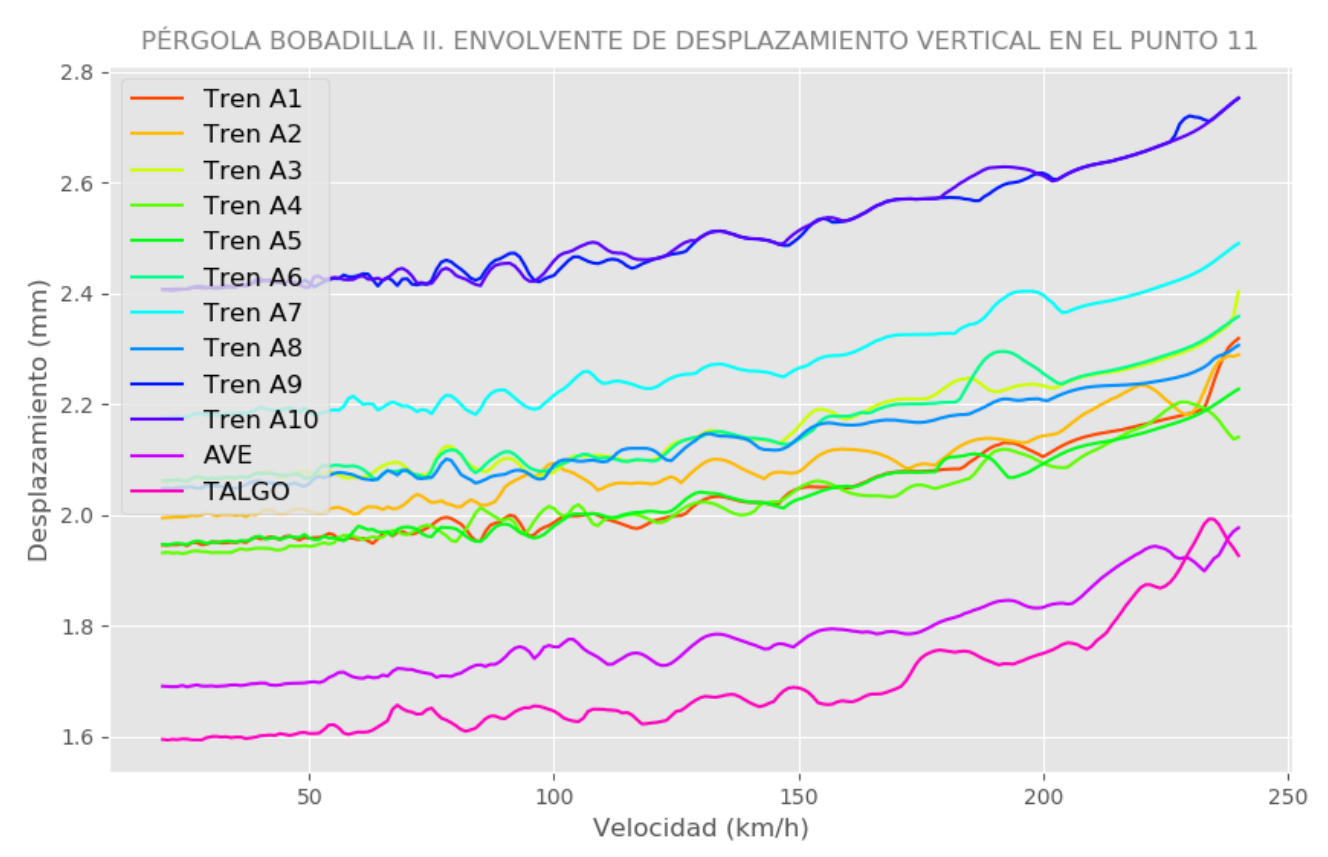

Figura 4.21: Envolvente de desplazamiento en el punto 11 para $E_{p} / G_{s}=250$. Pérgola de Bobadilla II

Estructura con $E_{p} / G_{s}=500$

$E_{p} / G_{s}=500$. Análisis modal

Los modos de vibración con frecuencia inferior a $30 \mathrm{~Hz}$ y su amortiguamiento por la interacción suelo estructura se pueden observar en la tabla 4.10.

\begin{tabular}{|c|c|c|c|}
\hline Modo & Período (s) & Frecuencia $(\mathrm{Hz})$ & Amortiguamiento por interacción suelo - estructura \\
\hline \hline 1 & 0.1576 & 6.3463 & 0.001824 \\
\hline 2 & 0.1536 & 6.5124 & 0.001988 \\
\hline 3 & 0.1483 & 6.7440 & 0.001884 \\
\hline 4 & 0.1432 & 6.9842 & 0.001828 \\
\hline 5 & 0.1399 & 7.1497 & 0.000234 \\
\hline 6 & 0.1395 & 7.1661 & 0.000082 \\
\hline 7 & 0.1373 & 7.2852 & 0.001583 \\
\hline 8 & 0.1319 & 7.5817 & 0.001652 \\
\hline 9 & 0.1275 & 7.8444 & 0.001517 \\
\hline 10 & 0.1254 & 7.9769 & 0.000759 \\
\hline 11 & 0.1242 & 8.0497 & 0.000560 \\
\hline 12 & 0.1218 & 8.2136 & 0.000927 \\
\hline 13 & 0.1205 & 8.3003 & 0.000819 \\
\hline
\end{tabular}

Continúa en la página siguiente. 


\begin{tabular}{|c|c|c|c|}
\hline Modo & Período (s) & Frecuencia $(\mathrm{Hz})$ & Amortiguamiento por interacción suelo - estructura \\
\hline 14 & 0.1154 & 8.6628 & 0.001466 \\
\hline 15 & 0.1140 & 8.7737 & 0.000801 \\
\hline 16 & 0.1105 & 9.0473 & 0.001348 \\
\hline 17 & 0.1092 & 9.1549 & 0.000694 \\
\hline 18 & 0.1062 & 9.4186 & 0.000593 \\
\hline 19 & 0.1034 & 9.6669 & 0.001189 \\
\hline 20 & 0.1004 & 9.9636 & 0.001480 \\
\hline 21 & 0.0975 & 10.2590 & 0.000655 \\
\hline 22 & 0.0952 & 10.5020 & 0.000918 \\
\hline 23 & 0.0931 & 10.7360 & 0.000732 \\
\hline 24 & 0.0896 & 11.1590 & 0.001604 \\
\hline 25 & 0.0892 & 11.2120 & 0.000976 \\
\hline 26 & 0.0865 & 11.5570 & 0.001687 \\
\hline 27 & 0.0851 & 11.7560 & 0.000751 \\
\hline 28 & 0.0824 & 12.1390 & 0.001555 \\
\hline 29 & 0.0815 & 12.2700 & 0.000946 \\
\hline 30 & 0.0783 & 12.7660 & 0.000594 \\
\hline 31 & 0.0765 & 13.0750 & 0.001823 \\
\hline 32 & 0.0735 & 13.6040 & 0.000473 \\
\hline 33 & 0.0715 & 13.9840 & 0.001978 \\
\hline 34 & 0.0710 & 14.0850 & 0.000565 \\
\hline 35 & 0.0694 & 14.4070 & 0.000985 \\
\hline 36 & 0.0673 & 14.8590 & 0.001847 \\
\hline 37 & 0.0667 & 14.9950 & 0.001045 \\
\hline 38 & 0.0656 & 15.2420 & 0.000846 \\
\hline 39 & 0.0638 & 15.6810 & 0.000575 \\
\hline 40 & 0.0630 & 15.8670 & 0.000655 \\
\hline 41 & 0.0615 & 16.2650 & 0.003026 \\
\hline 42 & 0.0595 & 16.8190 & 0.000632 \\
\hline 43 & 0.0586 & 17.0770 & 0.000548 \\
\hline 44 & 0.0570 & 17.5360 & 0.003177 \\
\hline 45 & 0.0564 & 17.7220 & 0.001124 \\
\hline 46 & 0.0560 & 17.8700 & 0.000653 \\
\hline 47 & 0.0542 & 18.4470 & 0.000997 \\
\hline 48 & 0.0527 & 18.9820 & 0.000609 \\
\hline 49 & 0.0516 & 19.3930 & 0.005838 \\
\hline 50 & 0.0511 & 19.5690 & 0.000858 \\
\hline
\end{tabular}

Continúa en la página siguiente. 


\begin{tabular}{|c|c|c|c|}
\hline Modo & Período (s) & Frecuencia $(\mathrm{Hz})$ & Amortiguamiento por interacción suelo - estructura \\
\hline 51 & 0.0506 & 19.7520 & 0.000437 \\
\hline 52 & 0.0503 & 19.9010 & 0.000674 \\
\hline 53 & 0.0475 & 21.0330 & 0.011181 \\
\hline 54 & 0.0467 & 21.4170 & 0.002187 \\
\hline 55 & 0.0462 & 21.6650 & 0.002236 \\
\hline 56 & 0.0453 & 22.0580 & 0.005970 \\
\hline 57 & 0.0444 & 22.5430 & 0.003248 \\
\hline 58 & 0.0438 & 22.8280 & 0.022654 \\
\hline 59 & 0.0436 & 22.9530 & 0.044558 \\
\hline 60 & 0.0430 & 23.2620 & 0.045142 \\
\hline 61 & 0.0427 & 23.4310 & 0.232704 \\
\hline 62 & 0.0425 & 23.5090 & 0.213629 \\
\hline 63 & 0.0423 & 23.6260 & 0.202033 \\
\hline 64 & 0.0421 & 23.7500 & 0.188981 \\
\hline 65 & 0.0419 & 23.8440 & 0.241858 \\
\hline 66 & 0.0418 & 23.9210 & 0.186965 \\
\hline 67 & 0.0417 & 23.9610 & 0.208366 \\
\hline 68 & 0.0417 & 23.9900 & 0.246941 \\
\hline 69 & 0.0414 & 24.1670 & 0.103868 \\
\hline 70 & 0.0412 & 24.2940 & 0.187529 \\
\hline 71 & 0.0409 & 24.4330 & 0.054398 \\
\hline 72 & 0.0408 & 24.5100 & 0.201574 \\
\hline 73 & 0.0406 & 24.6230 & 0.224183 \\
\hline 74 & 0.0404 & 24.7230 & 0.194861 \\
\hline 75 & 0.0403 & 24.8020 & 0.104127 \\
\hline 76 & 0.0401 & 24.9500 & 0.247654 \\
\hline 77 & 0.0399 & 25.0580 & 0.147081 \\
\hline 78 & 0.0399 & 25.0880 & 0.196986 \\
\hline 79 & 0.0395 & 25.3080 & 0.220281 \\
\hline 80 & 0.0394 & 25.3530 & 0.248966 \\
\hline 81 & 0.0392 & 25.4850 & 0.232906 \\
\hline 82 & 0.0392 & 25.5200 & 0.068337 \\
\hline 83 & 0.0391 & 25.5850 & 0.222785 \\
\hline 84 & 0.0389 & 25.6780 & 0.217829 \\
\hline 85 & 0.0388 & 25.7720 & 0.128281 \\
\hline 86 & 0.0388 & 25.7940 & 0.195506 \\
\hline 87 & 0.0386 & 25.8840 & 0.193817 \\
\hline
\end{tabular}

Continúa en la página siguiente. 


\begin{tabular}{|c|c|c|c|}
\hline Modo & Período (s) & Frecuencia $(\mathrm{Hz})$ & Amortiguamiento por interacción suelo - estructura \\
\hline 88 & 0.0384 & 26.0490 & 0.280084 \\
\hline 89 & 0.0383 & 26.0850 & 0.280043 \\
\hline 90 & 0.0383 & 26.0980 & 0.319093 \\
\hline 91 & 0.0383 & 26.1370 & 0.223895 \\
\hline 92 & 0.0382 & 26.1910 & 0.336972 \\
\hline 93 & 0.0381 & 26.2620 & 0.332574 \\
\hline 94 & 0.0380 & 26.2830 & 0.269234 \\
\hline 95 & 0.0380 & 26.3230 & 0.328746 \\
\hline 96 & 0.0379 & 26.4000 & 0.337794 \\
\hline 97 & 0.0378 & 26.4230 & 0.337685 \\
\hline 98 & 0.0378 & 26.4720 & 0.311850 \\
\hline 99 & 0.0377 & 26.5310 & 0.297452 \\
\hline 100 & 0.0377 & 26.5510 & 0.342435 \\
\hline 101 & 0.0376 & 26.5840 & 0.332824 \\
\hline 102 & 0.0375 & 26.6730 & 0.337755 \\
\hline 103 & 0.0375 & 26.6990 & 0.332890 \\
\hline 104 & 0.0374 & 26.7190 & 0.320216 \\
\hline 105 & 0.0373 & 26.7800 & 0.337598 \\
\hline 106 & 0.0373 & 26.8190 & 0.099743 \\
\hline 107 & 0.0372 & 26.8780 & 0.265014 \\
\hline 108 & 0.0372 & 26.9010 & 0.312488 \\
\hline 109 & 0.0371 & 26.9670 & 0.294563 \\
\hline 110 & 0.0370 & 27.0130 & 0.326758 \\
\hline 111 & 0.0369 & 27.1080 & 0.323709 \\
\hline 112 & 0.0368 & 27.1590 & 0.307664 \\
\hline 113 & 0.0368 & 27.2100 & 0.333505 \\
\hline 114 & 0.0366 & 27.3280 & 0.304109 \\
\hline 115 & 0.0365 & 27.4170 & 0.324658 \\
\hline 116 & 0.0363 & 27.5490 & 0.314962 \\
\hline 117 & 0.0363 & 27.5700 & 0.249636 \\
\hline 118 & 0.0362 & 27.6240 & 0.108311 \\
\hline 119 & 0.0359 & 27.8390 & 0.302843 \\
\hline 120 & 0.0359 & 27.8860 & 0.287307 \\
\hline 121 & 0.0358 & 27.9000 & 0.242833 \\
\hline 122 & 0.0357 & 27.9840 & 0.286027 \\
\hline 123 & 0.0357 & 28.0460 & 0.035236 \\
\hline 124 & 0.0356 & 28.0660 & 0.300842 \\
\hline
\end{tabular}

Continúa en la página siguiente. 


\begin{tabular}{|c|c|c|c|}
\hline Modo & Período (s) & Frecuencia $(\mathrm{Hz})$ & Amortiguamiento por interacción suelo - estructura \\
\hline \hline 125 & 0.0353 & 28.3140 & 0.172610 \\
\hline 126 & 0.0353 & 28.3430 & 0.142765 \\
\hline 127 & 0.0351 & 28.4980 & 0.304703 \\
\hline 128 & 0.0349 & 28.6230 & 0.235880 \\
\hline 129 & 0.0349 & 28.6520 & 0.042451 \\
\hline 130 & 0.0348 & 28.7320 & 0.049973 \\
\hline 131 & 0.0347 & 28.8040 & 0.088742 \\
\hline 132 & 0.0347 & 28.8250 & 0.227901 \\
\hline 133 & 0.0346 & 28.8770 & 0.289942 \\
\hline 134 & 0.0345 & 29.0270 & 0.052101 \\
\hline 135 & 0.0344 & 29.0810 & 0.300759 \\
\hline 136 & 0.0343 & 29.1600 & 0.298307 \\
\hline 137 & 0.0338 & 29.5600 & 0.158792 \\
\hline 138 & 0.0337 & 29.6450 & 0.114719 \\
\hline 139 & 0.0336 & 29.7600 & 0.317764 \\
\hline 140 & 0.0334 & 29.9480 & 0.315422 \\
\hline
\end{tabular}

Cuadro 4.10: Valores de período, frecuencia y amortiguamiento para $E_{p} / G_{s}=500$. Pérgola de Bobadilla II

$E_{p} / G_{s}=500$. Resultados de aceleración

En la tabla 4.11 se puede observar toda la información relativa al punto de aceleración máxima.

\begin{tabular}{|c|c|}
\hline Máximo valor de aceleración & $3.450 \mathrm{~m} / \mathrm{s}^{2}$ \\
\hline Punto & 2 \\
\hline Tren & 2 \\
\hline Velocidad & $240 \mathrm{~km} / \mathrm{h}$ \\
\hline
\end{tabular}

Cuadro 4.11: Máxima aceleración para $E_{p} / G_{s}=500$. Pérgola de Bobadilla II

En la figura 4.22 se puede observar la envolvente de aceleración en el punto en el que se alcanza su valor máximo. 


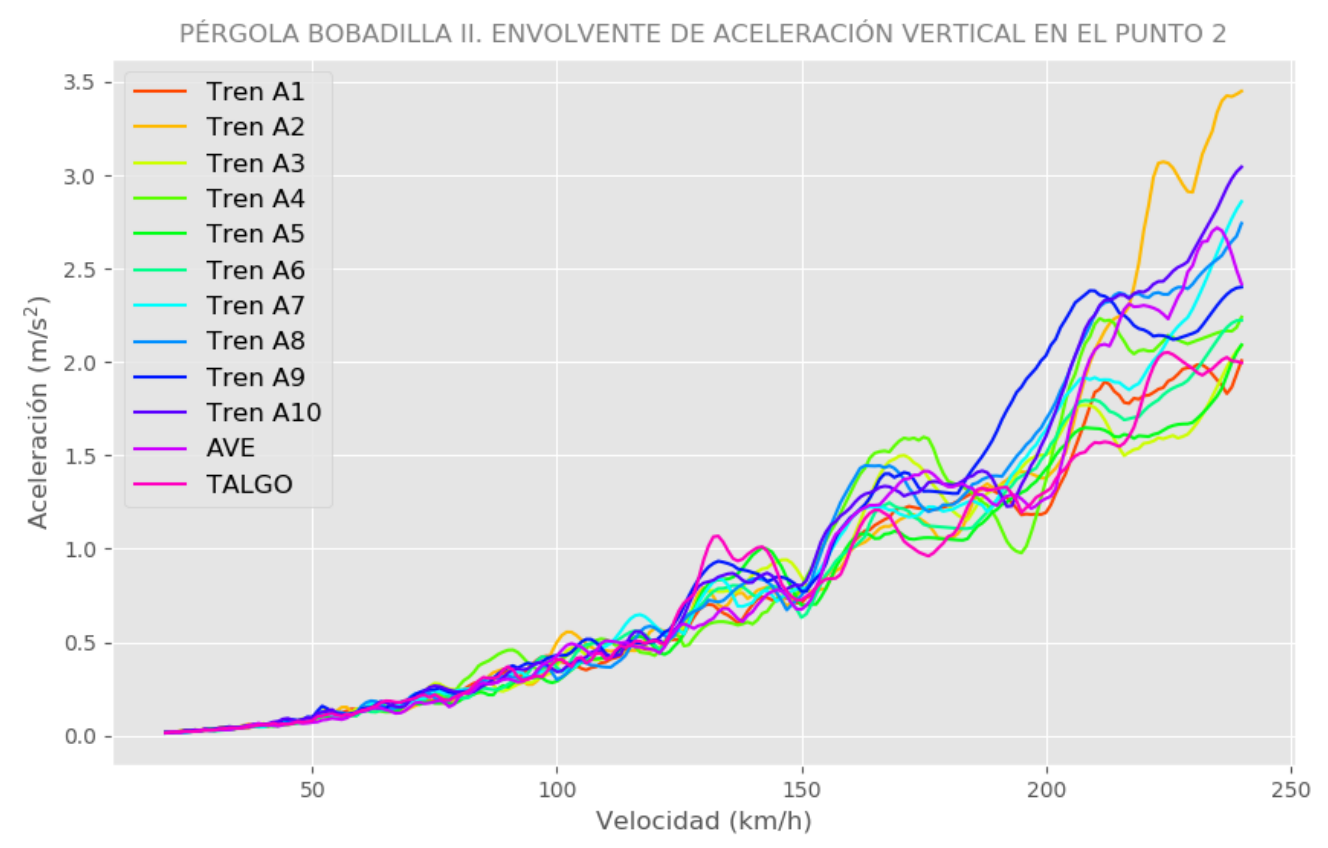

Figura 4.22: Envolvente de aceleración en el punto 2 para $E_{p} / G_{s}=500$. Pérgola de Bobadilla II

$E_{p} / G_{s}=500$. Resultados de desplazamiento

En la tabla 4.12 se puede observar toda la información relativa al punto de desplazamiento máximo.

\begin{tabular}{|c|c|}
\hline Máximo valor de desplazamiento & $2.768 \mathrm{~mm}$ \\
\hline Punto & 11 \\
\hline Tren & 9 \\
\hline Velocidad & $240 \mathrm{~km} / \mathrm{h}$ \\
\hline
\end{tabular}

Cuadro 4.12: Máximo desplazamiento para $E_{p} / G_{s}=500$. Pérgola de Bobadilla II

En la figura 4.23 se puede observar la envolvente de desplazamiento en el punto en el que se alcanza su valor máximo. 


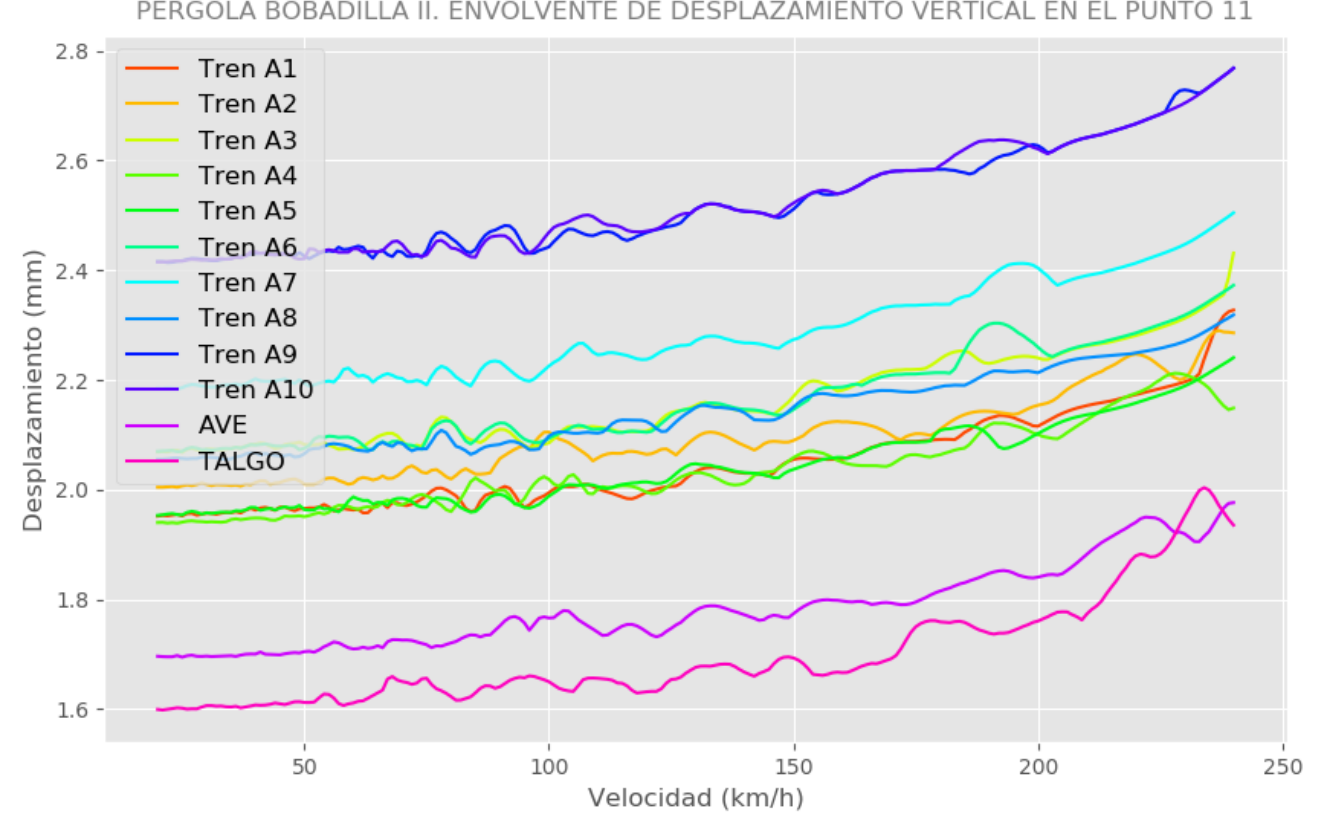

Figura 4.23: Envolvente de desplazamiento en el punto 11 para $E_{p} / G_{s}=500$. Pérgola de Bobadilla II

Estructura con $E_{p} / G_{s}=1000$

$E_{p} / G_{s}=1000$. Análisis modal

Los modos de vibración con frecuencia inferior a $30 \mathrm{~Hz}$ y su amortiguamiento por la interacción suelo estructura se pueden observar en la tabla 4.13 .

\begin{tabular}{|c|c|c|c|}
\hline Modo & Período (s) & Frecuencia $(\mathrm{Hz})$ & Amortiguamiento por interacción suelo - estructura \\
\hline \hline 1 & 0.1584 & 6.3126 & 0.003505 \\
\hline 2 & 0.1544 & 6.4757 & 0.003825 \\
\hline 3 & 0.1491 & 6.7092 & 0.003638 \\
\hline 4 & 0.1439 & 6.9503 & 0.003537 \\
\hline 5 & 0.1400 & 7.1444 & 0.000663 \\
\hline 6 & 0.1396 & 7.1646 & 0.000146 \\
\hline 7 & 0.1378 & 7.2570 & 0.002808 \\
\hline 8 & 0.1324 & 7.5514 & 0.003127 \\
\hline 9 & 0.1279 & 7.8162 & 0.002958 \\
\hline 10 & 0.1256 & 7.9616 & 0.001732 \\
\hline 11 & 0.1244 & 8.0388 & 0.001186 \\
\hline 12 & 0.1220 & 8.1970 & 0.001659 \\
\hline 13 & 0.1207 & 8.2854 & 0.001531 \\
\hline
\end{tabular}

Continúa en la página siguiente. 


\begin{tabular}{|c|c|c|c|}
\hline Modo & Período (s) & Frecuencia $(\mathrm{Hz})$ & Amortiguamiento por interacción suelo - estructura \\
\hline 14 & 0.1158 & 8.6353 & 0.002900 \\
\hline 15 & 0.1142 & 8.7586 & 0.001601 \\
\hline 16 & 0.1108 & 9.0217 & 0.002736 \\
\hline 17 & 0.1094 & 9.1416 & 0.001429 \\
\hline 18 & 0.1063 & 9.4074 & 0.001184 \\
\hline 19 & 0.1037 & 9.6448 & 0.002325 \\
\hline 20 & 0.1007 & 9.9354 & 0.003020 \\
\hline 21 & 0.0976 & 10.2460 & 0.001489 \\
\hline 22 & 0.0954 & 10.4840 & 0.001872 \\
\hline 23 & 0.0933 & 10.7230 & 0.001352 \\
\hline 24 & 0.0899 & 11.1260 & 0.003797 \\
\hline 25 & 0.0893 & 11.1950 & 0.001714 \\
\hline 26 & 0.0868 & 11.5230 & 0.003925 \\
\hline 27 & 0.0852 & 11.7430 & 0.001370 \\
\hline 28 & 0.0826 & 12.1060 & 0.003893 \\
\hline 29 & 0.0816 & 12.2540 & 0.001585 \\
\hline 30 & 0.0784 & 12.7530 & 0.001504 \\
\hline 31 & 0.0767 & 13.0390 & 0.004032 \\
\hline 32 & 0.0736 & 13.5950 & 0.001072 \\
\hline 33 & 0.0717 & 13.9410 & 0.005145 \\
\hline 34 & 0.0711 & 14.0730 & 0.001362 \\
\hline 35 & 0.0695 & 14.3870 & 0.002234 \\
\hline 36 & 0.0675 & 14.8160 & 0.005613 \\
\hline 37 & 0.0668 & 14.9740 & 0.002411 \\
\hline 38 & 0.0657 & 15.2250 & 0.001915 \\
\hline 39 & 0.0638 & 15.6680 & 0.001781 \\
\hline 40 & 0.0631 & 15.8510 & 0.002384 \\
\hline 41 & 0.0617 & 16.1980 & 0.008427 \\
\hline 42 & 0.0595 & 16.8030 & 0.002489 \\
\hline 43 & 0.0586 & 17.0620 & 0.002223 \\
\hline 44 & 0.0573 & 17.4510 & 0.012368 \\
\hline 45 & 0.0565 & 17.6950 & 0.003818 \\
\hline 46 & 0.0560 & 17.8560 & 0.001979 \\
\hline 47 & 0.0543 & 18.4110 & 0.007763 \\
\hline 48 & 0.0528 & 18.9570 & 0.006017 \\
\hline 49 & 0.0520 & 19.2180 & 0.028258 \\
\hline 50 & 0.0512 & 19.5350 & 0.011588 \\
\hline
\end{tabular}

Continúa en la página siguiente. 


\begin{tabular}{|c|c|c|c|}
\hline Modo & Período (s) & Frecuencia (Hz) & Amortiguamiento por interacción suelo - estructura \\
\hline 51 & 0.0507 & 19.7140 & 0.086852 \\
\hline 52 & 0.0506 & 19.7640 & 0.292977 \\
\hline 53 & 0.0505 & 19.7880 & 0.205306 \\
\hline 54 & 0.0503 & 19.8760 & 0.030018 \\
\hline 55 & 0.0498 & 20.0780 & 0.292377 \\
\hline 56 & 0.0497 & 20.1060 & 0.303185 \\
\hline 57 & 0.0497 & 20.1370 & 0.292001 \\
\hline 58 & 0.0495 & 20.1940 & 0.289827 \\
\hline 59 & 0.0490 & 20.4010 & 0.293309 \\
\hline 60 & 0.0488 & 20.4870 & 0.285321 \\
\hline 61 & 0.0485 & 20.6240 & 0.086790 \\
\hline 62 & 0.0484 & 20.6790 & 0.295586 \\
\hline 63 & 0.0480 & 20.8270 & 0.315754 \\
\hline 64 & 0.0479 & 20.8680 & 0.302616 \\
\hline 65 & 0.0478 & 20.9100 & 0.300327 \\
\hline 66 & 0.0474 & 21.1010 & 0.300797 \\
\hline 67 & 0.0472 & 21.2040 & 0.292860 \\
\hline 68 & 0.0470 & 21.2570 & 0.310289 \\
\hline 69 & 0.0469 & 21.3230 & 0.234141 \\
\hline 70 & 0.0468 & 21.3650 & 0.254879 \\
\hline 71 & 0.0467 & 21.4280 & 0.323906 \\
\hline 72 & 0.0466 & 21.4560 & 0.340476 \\
\hline 73 & 0.0466 & 21.4660 & 0.337549 \\
\hline 74 & 0.0465 & 21.4930 & 0.321357 \\
\hline 75 & 0.0465 & 21.5190 & 0.294979 \\
\hline 76 & 0.0464 & 21.5730 & 0.320257 \\
\hline 77 & 0.0463 & 21.6040 & 0.293211 \\
\hline 78 & 0.0462 & 21.6620 & 0.221975 \\
\hline 79 & 0.0461 & 21.6830 & 0.241615 \\
\hline 80 & 0.0461 & 21.7140 & 0.295829 \\
\hline 81 & 0.0459 & 21.7860 & 0.329583 \\
\hline 82 & 0.0459 & 21.7870 & 0.299658 \\
\hline 83 & 0.0459 & 21.7940 & 0.190021 \\
\hline 84 & 0.0458 & 21.8530 & 0.313451 \\
\hline 85 & 0.0457 & 21.8790 & 0.326252 \\
\hline 86 & 0.0456 & 21.9240 & 0.305158 \\
\hline 87 & 0.0455 & 21.9840 & 0.329588 \\
\hline
\end{tabular}

Continúa en la página siguiente. 


\begin{tabular}{|c|c|c|c|}
\hline Modo & Período (s) & Frecuencia $(\mathrm{Hz})$ & Amortiguamiento por interacción suelo - estructura \\
\hline 88 & 0.0453 & 22.0760 & 0.335220 \\
\hline 89 & 0.0452 & 22.1160 & 0.283305 \\
\hline 90 & 0.0451 & 22.1900 & 0.192457 \\
\hline 91 & 0.0450 & 22.2150 & 0.322459 \\
\hline 92 & 0.0449 & 22.2520 & 0.294001 \\
\hline 93 & 0.0449 & 22.2820 & 0.301793 \\
\hline 94 & 0.0447 & 22.3820 & 0.278633 \\
\hline 95 & 0.0446 & 22.4110 & 0.307215 \\
\hline 96 & 0.0446 & 22.4440 & 0.291822 \\
\hline 97 & 0.0444 & 22.5320 & 0.219746 \\
\hline 98 & 0.0442 & 22.6420 & 0.142491 \\
\hline 99 & 0.0440 & 22.7020 & 0.301577 \\
\hline 100 & 0.0440 & 22.7360 & 0.315303 \\
\hline 101 & 0.0438 & 22.8500 & 0.303254 \\
\hline 102 & 0.0436 & 22.9510 & 0.285328 \\
\hline 103 & 0.0436 & 22.9600 & 0.301902 \\
\hline 104 & 0.0434 & 23.0350 & 0.315051 \\
\hline 105 & 0.0433 & 23.1000 & 0.290906 \\
\hline 106 & 0.0430 & 23.2480 & 0.067453 \\
\hline 107 & 0.0429 & 23.3080 & 0.261706 \\
\hline 108 & 0.0428 & 23.3810 & 0.273651 \\
\hline 109 & 0.0426 & 23.4710 & 0.133014 \\
\hline 110 & 0.0425 & 23.5430 & 0.256496 \\
\hline 111 & 0.0421 & 23.7560 & 0.272033 \\
\hline 112 & 0.0418 & 23.9190 & 0.293572 \\
\hline 113 & 0.0414 & 24.1620 & 0.153128 \\
\hline 114 & 0.0413 & 24.2140 & 0.138822 \\
\hline 115 & 0.0410 & 24.3690 & 0.206038 \\
\hline 116 & 0.0408 & 24.4940 & 0.281963 \\
\hline 117 & 0.0406 & 24.6190 & 0.309524 \\
\hline 118 & 0.0403 & 24.7960 & 0.158512 \\
\hline 119 & 0.0403 & 24.8410 & 0.203403 \\
\hline 120 & 0.0402 & 24.8990 & 0.146410 \\
\hline 121 & 0.0400 & 24.9800 & 0.250034 \\
\hline 122 & 0.0398 & 25.1050 & 0.048751 \\
\hline 123 & 0.0396 & 25.2810 & 0.277359 \\
\hline 124 & 0.0391 & 25.5890 & 0.010097 \\
\hline
\end{tabular}

Continúa en la página siguiente. 


\begin{tabular}{|c|c|c|c|}
\hline Modo & Período (s) & Frecuencia $(\mathrm{Hz})$ & Amortiguamiento por interacción suelo - estructura \\
\hline \hline 125 & 0.0391 & 25.6020 & 0.299672 \\
\hline 126 & 0.0389 & 25.6820 & 0.078137 \\
\hline 127 & 0.0388 & 25.7960 & 0.240432 \\
\hline 128 & 0.0388 & 25.8060 & 0.062826 \\
\hline 129 & 0.0384 & 26.0530 & 0.003352 \\
\hline 130 & 0.0373 & 26.7780 & 0.001984 \\
\hline 131 & 0.0369 & 27.1340 & 0.032810 \\
\hline 132 & 0.0363 & 27.5780 & 0.002620 \\
\hline 133 & 0.0358 & 27.9400 & 0.004323 \\
\hline 134 & 0.0357 & 27.9910 & 0.055538 \\
\hline 135 & 0.0354 & 28.2460 & 0.007984 \\
\hline 136 & 0.0352 & 28.4370 & 0.024787 \\
\hline 137 & 0.0350 & 28.5610 & 0.015436 \\
\hline 138 & 0.0349 & 28.6920 & 0.003127 \\
\hline 139 & 0.0347 & 28.7930 & 0.019040 \\
\hline 140 & 0.0344 & 29.0550 & 0.015942 \\
\hline 141 & 0.0343 & 29.1440 & 0.015705 \\
\hline 142 & 0.0340 & 29.4400 & 0.055885 \\
\hline 143 & 0.0338 & 29.5650 & 0.034947 \\
\hline & & & \\
\hline
\end{tabular}

Cuadro 4.13: Valores de período, frecuencia y amortiguamiento para $E_{p} / G_{s}=1000$. Pérgola de Bobadilla II

$E_{p} / G_{s}=1000$. Resultados de aceleración

En la tabla 4.14 se puede observar toda la información relativa al punto de aceleración máxima.

\begin{tabular}{|c|c|}
\hline Máximo valor de aceleración & $2.707 \mathrm{~m} / \mathrm{s}^{2}$ \\
\hline Punto & 5 \\
\hline Tren & 9 \\
\hline Velocidad & $240 \mathrm{~km} / \mathrm{h}$ \\
\hline
\end{tabular}

Cuadro 4.14: Máxima aceleración para $E_{p} / G_{s}=1000$. Pérgola de Bobadilla II

En la figura 4.24 se puede observar la envolvente de aceleración en el punto en el que se alcanza su valor máximo. 


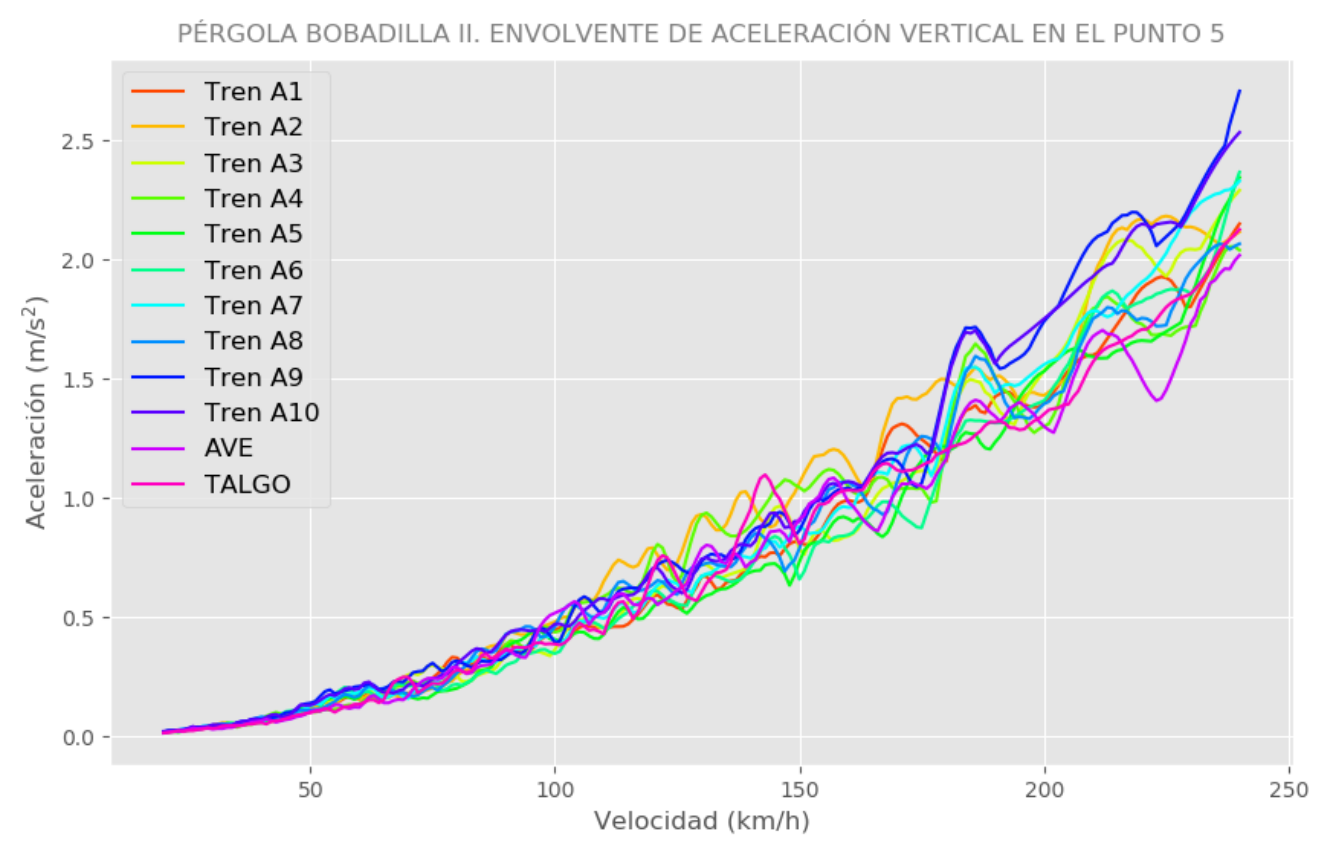

Figura 4.24: Envolvente de aceleración en el punto 5 para $E_{p} / G_{s}=1000$. Pérgola de Bobadilla II

$E_{p} / G_{s}=1000$. Resultados de desplazamiento

En la tabla 4.15 se puede observar toda la información relativa al punto de desplazamiento máximo.

\begin{tabular}{|c|c|}
\hline Máximo valor de desplazamiento & $2.798 \mathrm{~mm}$ \\
\hline Punto & 11 \\
\hline Tren & 9 \\
\hline Velocidad & $240 \mathrm{~km} / \mathrm{h}$ \\
\hline
\end{tabular}

Cuadro 4.15: Máximo desplazamiento para $E_{p} / G_{s}=1000$. Pérgola de Bobadilla II

En la figura 4.25 se puede observar la envolvente de desplazamiento en el punto en el que se alcanza su valor máximo. 


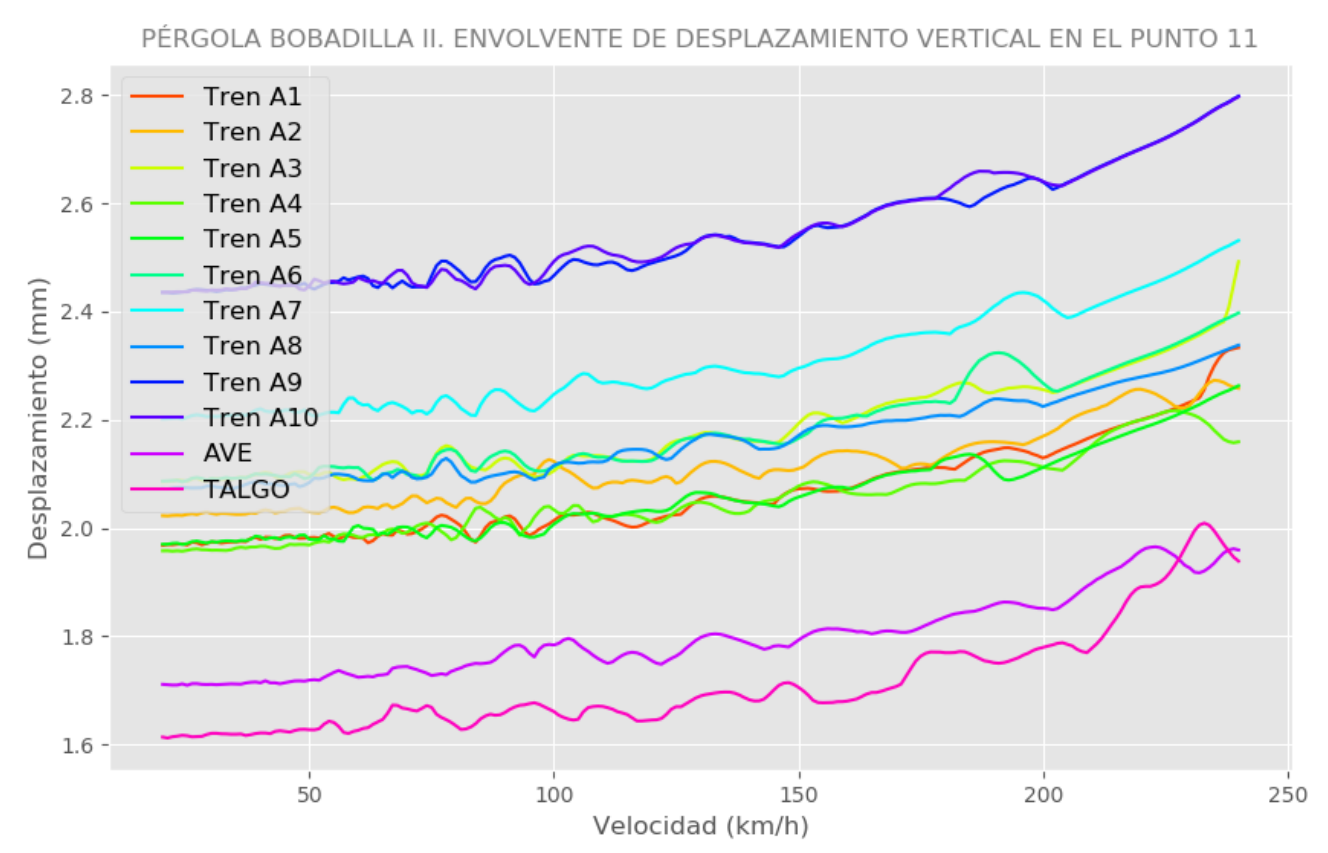

Figura 4.25: Envolvente de desplazamiento en el punto 11 para $E_{p} / G_{s}=1000$. Pérgola de Bobadilla II

Estructura con $E_{p} / G_{s}=2500$

$E_{p} / G_{s}=2500$. Análisis modal

Los modos de vibración con frecuencia inferior a $30 \mathrm{~Hz}$ y su amortiguamiento por la interacción suelo estructura se pueden observar en la tabla 4.16 .

\begin{tabular}{|c|c|c|c|}
\hline Modo & Período (s) & Frecuencia $(\mathrm{Hz})$ & Amortiguamiento por interacción suelo - estructura \\
\hline \hline 1 & 0.1612 & 6.2025 & 0.010574 \\
\hline 2 & 0.1573 & 6.3554 & 0.011562 \\
\hline 3 & 0.1517 & 6.5941 & 0.011138 \\
\hline 4 & 0.1462 & 6.8382 & 0.010863 \\
\hline 5 & 0.1408 & 7.1001 & 0.007007 \\
\hline 6 & 0.1397 & 7.1601 & 0.000451 \\
\hline 7 & 0.1390 & 7.1925 & 0.003471 \\
\hline 8 & 0.1341 & 7.4545 & 0.009227 \\
\hline 9 & 0.1295 & 7.7200 & 0.009606 \\
\hline 10 & 0.1267 & 7.8912 & 0.008335 \\
\hline 11 & 0.1251 & 7.9948 & 0.004984 \\
\hline 12 & 0.1227 & 8.1511 & 0.003868 \\
\hline 13 & 0.1214 & 8.2401 & 0.004028 \\
\hline
\end{tabular}

Continúa en la página siguiente. 


\begin{tabular}{|c|c|c|c|}
\hline Modo & Período (s) & Frecuencia $(\mathrm{Hz})$ & Amortiguamiento por interacción suelo - estructura \\
\hline 14 & 0.1171 & 8.5394 & 0.009705 \\
\hline 15 & 0.1149 & 8.7040 & 0.005753 \\
\hline 16 & 0.1120 & 8.9292 & 0.009432 \\
\hline 17 & 0.1100 & 9.0889 & 0.006019 \\
\hline 18 & 0.1068 & 9.3672 & 0.004194 \\
\hline 19 & 0.1045 & 9.5701 & 0.007310 \\
\hline 20 & 0.1017 & 9.8311 & 0.010928 \\
\hline 21 & 0.0982 & 10.1810 & 0.008438 \\
\hline 22 & 0.0959 & 10.4230 & 0.005755 \\
\hline 23 & 0.0936 & 10.6810 & 0.004243 \\
\hline 24 & 0.0911 & 10.9770 & 0.017145 \\
\hline 25 & 0.0898 & 11.1330 & 0.007449 \\
\hline 26 & 0.0881 & 11.3570 & 0.020440 \\
\hline 27 & 0.0855 & 11.7020 & 0.003983 \\
\hline 28 & 0.0838 & 11.9290 & 0.022820 \\
\hline 29 & 0.0819 & 12.2110 & 0.003954 \\
\hline 30 & 0.0790 & 12.6560 & 0.017972 \\
\hline 31 & 0.0776 & 12.8900 & 0.014825 \\
\hline 32 & 0.0739 & 13.5380 & 0.011277 \\
\hline 33 & 0.0732 & 13.6530 & 0.045151 \\
\hline 34 & 0.0717 & 13.9400 & 0.047076 \\
\hline 35 & 0.0702 & 14.2470 & 0.040286 \\
\hline 36 & 0.0697 & 14.3450 & 0.290536 \\
\hline 37 & 0.0697 & 14.3530 & 0.297499 \\
\hline 38 & 0.0694 & 14.4080 & 0.076405 \\
\hline 39 & 0.0687 & 14.5480 & 0.302591 \\
\hline 40 & 0.0687 & 14.5610 & 0.294567 \\
\hline 41 & 0.0680 & 14.7020 & 0.292246 \\
\hline 42 & 0.0677 & 14.7790 & 0.253269 \\
\hline 43 & 0.0673 & 14.8510 & 0.098376 \\
\hline 44 & 0.0671 & 14.8980 & 0.292551 \\
\hline 45 & 0.0665 & 15.0390 & 0.286040 \\
\hline 46 & 0.0662 & 15.1030 & 0.280935 \\
\hline 47 & 0.0661 & 15.1240 & 0.135955 \\
\hline 48 & 0.0659 & 15.1860 & 0.295142 \\
\hline 49 & 0.0658 & 15.2080 & 0.297471 \\
\hline 50 & 0.0654 & 15.2920 & 0.171061 \\
\hline
\end{tabular}

Continúa en la página siguiente. 


\begin{tabular}{|c|c|c|c|}
\hline Modo & Período (s) & Frecuencia (Hz) & Amortiguamiento por interacción suelo - estructura \\
\hline 51 & 0.0653 & 15.3160 & 0.275906 \\
\hline 52 & 0.0651 & 15.3650 & 0.297252 \\
\hline 53 & 0.0650 & 15.3790 & 0.303389 \\
\hline 54 & 0.0649 & 15.4020 & 0.307473 \\
\hline 55 & 0.0647 & 15.4540 & 0.308456 \\
\hline 56 & 0.0645 & 15.5010 & 0.252501 \\
\hline 57 & 0.0644 & 15.5370 & 0.297526 \\
\hline 58 & 0.0641 & 15.5910 & 0.279279 \\
\hline 59 & 0.0638 & 15.6660 & 0.286222 \\
\hline 60 & 0.0636 & 15.7120 & 0.290153 \\
\hline 61 & 0.0635 & 15.7440 & 0.290826 \\
\hline 62 & 0.0634 & 15.7830 & 0.258503 \\
\hline 63 & 0.0632 & 15.8110 & 0.275085 \\
\hline 64 & 0.0631 & 15.8470 & 0.226632 \\
\hline 65 & 0.0629 & 15.8920 & 0.217336 \\
\hline 66 & 0.0627 & 15.9470 & 0.246002 \\
\hline 67 & 0.0626 & 15.9720 & 0.275163 \\
\hline 68 & 0.0625 & 16.0020 & 0.204031 \\
\hline 69 & 0.0622 & 16.0870 & 0.261747 \\
\hline 70 & 0.0621 & 16.1100 & 0.281186 \\
\hline 71 & 0.0617 & 16.2090 & 0.282428 \\
\hline 72 & 0.0613 & 16.3230 & 0.286920 \\
\hline 73 & 0.0609 & 16.4290 & 0.276132 \\
\hline 74 & 0.0606 & 16.5020 & 0.282226 \\
\hline 75 & 0.0604 & 16.5590 & 0.270006 \\
\hline 76 & 0.0603 & 16.5890 & 0.246821 \\
\hline 77 & 0.0601 & 16.6490 & 0.255278 \\
\hline 78 & 0.0597 & 16.7600 & 0.277245 \\
\hline 79 & 0.0596 & 16.7850 & 0.210979 \\
\hline 80 & 0.0592 & 16.9030 & 0.238444 \\
\hline 81 & 0.0588 & 17.0100 & 0.115458 \\
\hline 82 & 0.0588 & 17.0160 & 0.255246 \\
\hline 83 & 0.0585 & 17.0880 & 0.215084 \\
\hline 84 & 0.0583 & 17.1590 & 0.147145 \\
\hline 85 & 0.0582 & 17.1910 & 0.252949 \\
\hline 86 & 0.0579 & 17.2620 & 0.241237 \\
\hline 87 & 0.0577 & 17.3270 & 0.257740 \\
\hline
\end{tabular}

Continúa en la página siguiente. 


\begin{tabular}{|c|c|c|c|}
\hline Modo & Período (s) & Frecuencia $(\mathrm{Hz})$ & Amortiguamiento por interacción suelo - estructura \\
\hline 88 & 0.0573 & 17.4590 & 0.280608 \\
\hline 89 & 0.0571 & 17.5030 & 0.274045 \\
\hline 90 & 0.0570 & 17.5530 & 0.233484 \\
\hline 91 & 0.0564 & 17.7230 & 0.019699 \\
\hline 92 & 0.0560 & 17.8690 & 0.037432 \\
\hline 93 & 0.0559 & 17.9000 & 0.259632 \\
\hline 94 & 0.0554 & 18.0410 & 0.203012 \\
\hline 95 & 0.0552 & 18.1080 & 0.255028 \\
\hline 96 & 0.0547 & 18.2830 & 0.223768 \\
\hline 97 & 0.0541 & 18.4700 & 0.203178 \\
\hline 98 & 0.0540 & 18.5270 & 0.049351 \\
\hline 99 & 0.0537 & 18.6210 & 0.169020 \\
\hline 100 & 0.0531 & 18.8280 & 0.233866 \\
\hline 101 & 0.0525 & 19.0400 & 0.014587 \\
\hline 102 & 0.0517 & 19.3360 & 0.244288 \\
\hline 103 & 0.0515 & 19.4360 & 0.258517 \\
\hline 104 & 0.0511 & 19.5830 & 0.026280 \\
\hline 105 & 0.0509 & 19.6370 & 0.239274 \\
\hline 106 & 0.0507 & 19.7270 & 0.228090 \\
\hline 107 & 0.0506 & 19.7530 & 0.022213 \\
\hline 108 & 0.0504 & 19.8580 & 0.014939 \\
\hline 109 & 0.0498 & 20.0910 & 0.048556 \\
\hline 110 & 0.0492 & 20.3160 & 0.223103 \\
\hline 111 & 0.0485 & 20.6030 & 0.253722 \\
\hline 112 & 0.0483 & 20.6850 & 0.228206 \\
\hline 113 & 0.0480 & 20.8220 & 0.251758 \\
\hline 114 & 0.0478 & 20.9410 & 0.188850 \\
\hline 115 & 0.0466 & 21.4720 & 0.007183 \\
\hline 116 & 0.0464 & 21.5340 & 0.014613 \\
\hline 117 & 0.0460 & 21.7530 & 0.007121 \\
\hline 118 & 0.0452 & 22.1320 & 0.001433 \\
\hline 119 & 0.0443 & 22.5840 & 0.000617 \\
\hline 120 & 0.0431 & 23.1890 & 0.002606 \\
\hline 121 & 0.0427 & 23.4200 & 0.011295 \\
\hline 122 & 0.0426 & 23.4740 & 0.002981 \\
\hline 123 & 0.0413 & 24.2180 & 0.001258 \\
\hline 124 & 0.0403 & 24.8120 & 0.000886 \\
\hline
\end{tabular}

Continúa en la página siguiente. 


\begin{tabular}{|c|c|c|c|}
\hline Modo & Período (s) & Frecuencia $(\mathrm{Hz})$ & Amortiguamiento por interacción suelo - estructura \\
\hline \hline 125 & 0.0400 & 25.0210 & 0.001105 \\
\hline 126 & 0.0396 & 25.2410 & 0.007486 \\
\hline 127 & 0.0391 & 25.5800 & 0.000256 \\
\hline 128 & 0.0388 & 25.7800 & 0.000500 \\
\hline 129 & 0.0384 & 26.0310 & 0.000529 \\
\hline 130 & 0.0374 & 26.7610 & 0.000496 \\
\hline 131 & 0.0372 & 26.8900 & 0.005390 \\
\hline 132 & 0.0365 & 27.4270 & 0.016145 \\
\hline 133 & 0.0363 & 27.5500 & 0.001588 \\
\hline 134 & 0.0359 & 27.8910 & 0.002344 \\
\hline 135 & 0.0357 & 27.9870 & 0.012293 \\
\hline 136 & 0.0354 & 28.2430 & 0.002759 \\
\hline 137 & 0.0352 & 28.4480 & 0.003192 \\
\hline 138 & 0.0350 & 28.6080 & 0.005874 \\
\hline 139 & 0.0349 & 28.6490 & 0.003107 \\
\hline 140 & 0.0346 & 28.8650 & 0.014756 \\
\hline 141 & 0.0346 & 28.9250 & 0.004529 \\
\hline 142 & 0.0345 & 29.0230 & 0.003491 \\
\hline 143 & 0.0342 & 29.2480 & 0.008032 \\
\hline 144 & 0.0337 & 29.6440 & 0.013310 \\
\hline 145 & 0.0334 & 29.9240 & 0.012643 \\
\hline & & & 0 \\
\hline
\end{tabular}

Cuadro 4.16: Valores de período, frecuencia y amortiguamiento para $E_{p} / G_{s}=2500$. Pérgola de Bobadilla II

$E_{p} / G_{s}=2500$. Resultados de aceleración

En la tabla 4.17 se puede observar toda la información relativa al punto de aceleración máxima.

\begin{tabular}{|c|c|}
\hline Máximo valor de aceleración & $2.857 \mathrm{~m} / \mathrm{s}^{2}$ \\
\hline Punto & 5 \\
\hline Tren & 9 \\
\hline Velocidad & $240 \mathrm{~km} / \mathrm{h}$ \\
\hline
\end{tabular}

Cuadro 4.17: Máxima aceleración para $E_{p} / G_{s}=2500$. Pérgola de Bobadilla II

En la figura 4.26 se puede observar la envolvente de aceleración en el punto en el que se alcanza su valor máximo. 


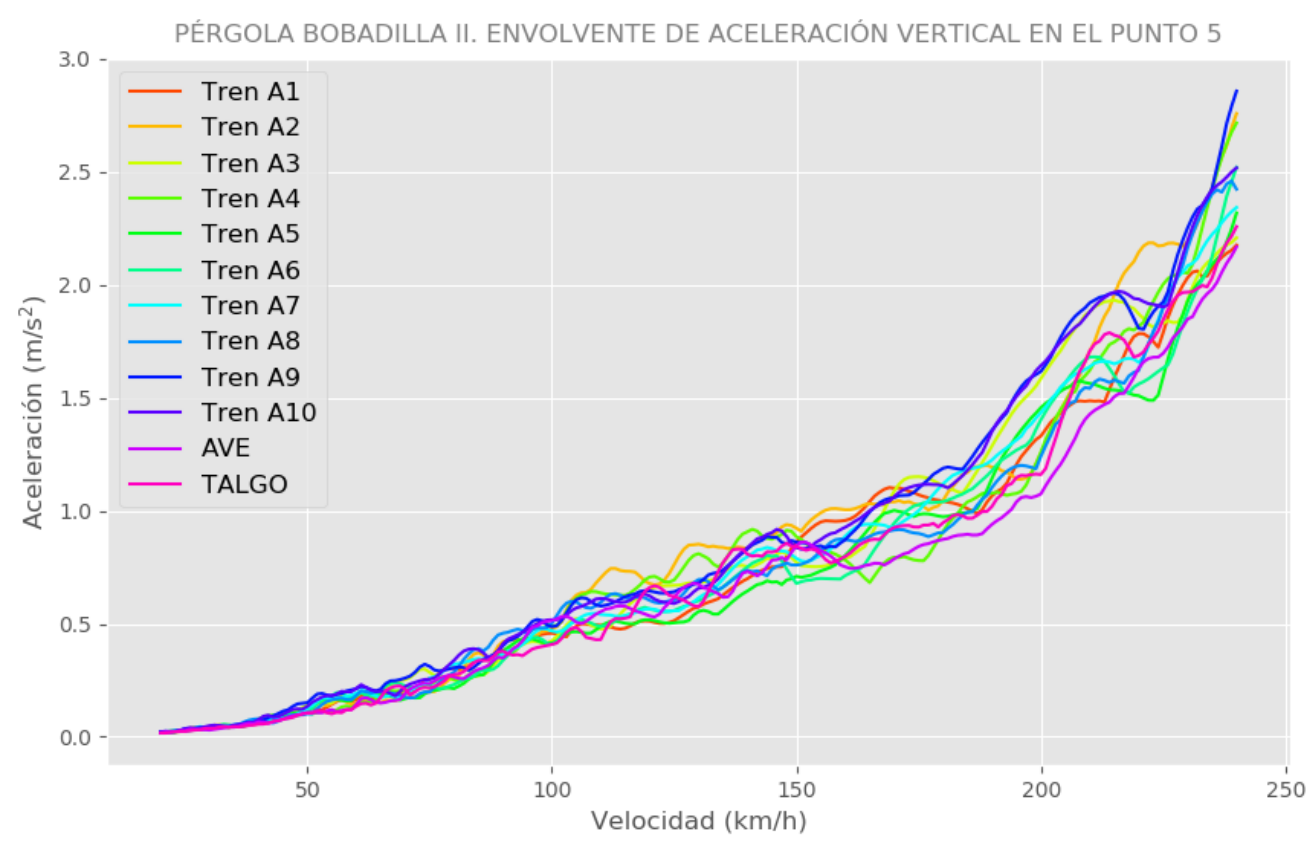

Figura 4.26: Envolvente de aceleración en el punto 5 para $E_{p} / G_{s}=2500$. Pérgola de Bobadilla II

$E_{p} / G_{s}=2500$. Resultados de desplazamiento

En la tabla 4.18 se puede observar toda la información relativa al punto de desplazamiento máximo.

\begin{tabular}{|c|c|}
\hline Máximo valor de desplazamiento & $2.843 \mathrm{~mm}$ \\
\hline Punto & 11 \\
\hline Tren & 10 \\
\hline Velocidad & $240 \mathrm{~km} / \mathrm{h}$ \\
\hline
\end{tabular}

Cuadro 4.18: Máximo desplazamiento para $E_{p} / G_{s}=2500$. Pérgola de Bobadilla II

En la figura 4.27 se puede observar la envolvente de desplazamiento en el punto en el que se alcanza su valor máximo. 


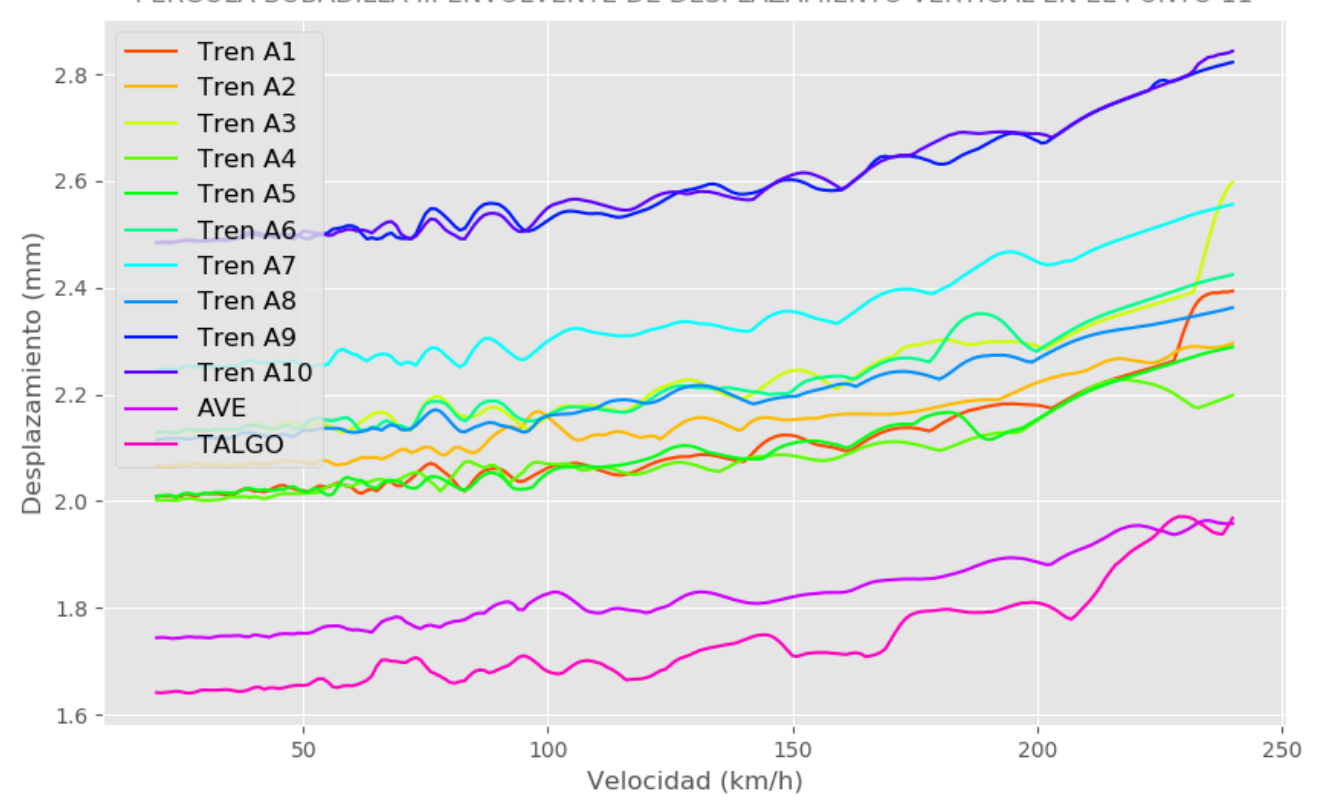

Figura 4.27: Envolvente de desplazamiento en el punto 11 para $E_{p} / G_{s}=2500$. Pérgola de Bobadilla II

Estructura con $E_{p} / G_{s}=5000$

$E_{p} / G_{s}=5000$. Análisis modal

Los modos de vibración con frecuencia inferior a $30 \mathrm{~Hz}$ y su amortiguamiento por la interacción suelo estructura se pueden observar en la tabla 4.19.

\begin{tabular}{|c|c|c|c|}
\hline Modo & Período (s) & Frecuencia $(\mathrm{Hz})$ & Amortiguamiento por interacción suelo - estructura \\
\hline \hline 1 & 0.1683 & 5.9427 & 0.034220 \\
\hline 2 & 0.1662 & 6.0163 & 0.000175 \\
\hline 3 & 0.1647 & 6.0720 & 0.037053 \\
\hline 4 & 0.1641 & 6.0923 & 0.000138 \\
\hline 5 & 0.1583 & 6.3168 & 0.036885 \\
\hline 6 & 0.1523 & 6.5676 & 0.035981 \\
\hline 7 & 0.1456 & 6.8691 & 0.033130 \\
\hline 8 & 0.1402 & 7.1349 & 0.009103 \\
\hline 9 & 0.1396 & 7.1615 & 0.002081 \\
\hline 10 & 0.1382 & 7.2375 & 0.024048 \\
\hline 11 & 0.1342 & 7.4519 & 0.040537 \\
\hline 12 & 0.1314 & 7.6088 & 0.045808 \\
\hline 13 & 0.1281 & 7.8077 & 0.033253 \\
\hline
\end{tabular}

Continúa en la página siguiente. 


\begin{tabular}{|c|c|c|c|}
\hline Modo & Período (s) & Frecuencia $(\mathrm{Hz})$ & Amortiguamiento por interacción suelo - estructura \\
\hline 14 & 0.1240 & 8.0629 & 0.015199 \\
\hline 15 & 0.1225 & 8.1601 & 0.009496 \\
\hline 16 & 0.1207 & 8.2841 & 0.031650 \\
\hline 17 & 0.1177 & 8.4955 & 0.040217 \\
\hline 18 & 0.1149 & 8.7004 & 0.025873 \\
\hline 19 & 0.1130 & 8.8518 & 0.044292 \\
\hline 20 & 0.1083 & 9.2302 & 0.029110 \\
\hline 21 & 0.1068 & 9.3594 & 0.033418 \\
\hline 22 & 0.1047 & 9.5543 & 0.029199 \\
\hline 23 & 0.1021 & 9.7932 & 0.074545 \\
\hline 24 & 0.0977 & 10.2370 & 0.105269 \\
\hline 25 & 0.0973 & 10.2750 & 0.176203 \\
\hline 26 & 0.0968 & 10.3330 & 0.226077 \\
\hline 27 & 0.0964 & 10.3740 & 0.198277 \\
\hline 28 & 0.0962 & 10.3990 & 0.275702 \\
\hline 29 & 0.0956 & 10.4560 & 0.281202 \\
\hline 30 & 0.0951 & 10.5180 & 0.291905 \\
\hline 31 & 0.0950 & 10.5290 & 0.159797 \\
\hline 32 & 0.0942 & 10.6170 & 0.123049 \\
\hline 33 & 0.0937 & 10.6760 & 0.242704 \\
\hline 34 & 0.0925 & 10.8050 & 0.292782 \\
\hline 35 & 0.0923 & 10.8370 & 0.153572 \\
\hline 36 & 0.0914 & 10.9440 & 0.244851 \\
\hline 37 & 0.0910 & 10.9940 & 0.217561 \\
\hline 38 & 0.0907 & 11.0190 & 0.262000 \\
\hline 39 & 0.0900 & 11.1060 & 0.277317 \\
\hline 40 & 0.0894 & 11.1820 & 0.273467 \\
\hline 41 & 0.0884 & 11.3100 & 0.271532 \\
\hline 42 & 0.0883 & 11.3240 & 0.269978 \\
\hline 43 & 0.0881 & 11.3500 & 0.202897 \\
\hline 44 & 0.0876 & 11.4130 & 0.258022 \\
\hline 45 & 0.0871 & 11.4850 & 0.237454 \\
\hline 46 & 0.0870 & 11.4930 & 0.254125 \\
\hline 47 & 0.0867 & 11.5360 & 0.176911 \\
\hline 48 & 0.0861 & 11.6200 & 0.221740 \\
\hline 49 & 0.0856 & 11.6840 & 0.215953 \\
\hline 50 & 0.0854 & 11.7070 & 0.250793 \\
\hline
\end{tabular}

Continúa en la página siguiente. 


\begin{tabular}{|c|c|c|c|}
\hline Modo & Período (s) & Frecuencia $(\mathrm{Hz})$ & Amortiguamiento por interacción suelo - estructura \\
\hline 51 & 0.0851 & 11.7570 & 0.251625 \\
\hline 52 & 0.0848 & 11.7930 & 0.183075 \\
\hline 53 & 0.0841 & 11.8950 & 0.237755 \\
\hline 54 & 0.0838 & 11.9350 & 0.226125 \\
\hline 55 & 0.0836 & 11.9560 & 0.246271 \\
\hline 56 & 0.0832 & 12.0230 & 0.219833 \\
\hline 57 & 0.0822 & 12.1650 & 0.135544 \\
\hline 58 & 0.0819 & 12.2040 & 0.207359 \\
\hline 59 & 0.0816 & 12.2480 & 0.177643 \\
\hline 60 & 0.0811 & 12.3270 & 0.227873 \\
\hline 61 & 0.0809 & 12.3650 & 0.188301 \\
\hline 62 & 0.0802 & 12.4630 & 0.203531 \\
\hline 63 & 0.0799 & 12.5140 & 0.226681 \\
\hline 64 & 0.0790 & 12.6510 & 0.212876 \\
\hline 65 & 0.0789 & 12.6820 & 0.216508 \\
\hline 66 & 0.0781 & 12.8000 & 0.173345 \\
\hline 67 & 0.0776 & 12.8790 & 0.104228 \\
\hline 68 & 0.0767 & 13.0410 & 0.235114 \\
\hline 69 & 0.0762 & 13.1160 & 0.237537 \\
\hline 70 & 0.0760 & 13.1570 & 0.196144 \\
\hline 71 & 0.0754 & 13.2650 & 0.196266 \\
\hline 72 & 0.0748 & 13.3710 & 0.194729 \\
\hline 73 & 0.0742 & 13.4690 & 0.188234 \\
\hline 74 & 0.0739 & 13.5290 & 0.075609 \\
\hline 75 & 0.0738 & 13.5560 & 0.234147 \\
\hline 76 & 0.0736 & 13.5920 & 0.223148 \\
\hline 77 & 0.0724 & 13.8160 & 0.237866 \\
\hline 78 & 0.0722 & 13.8460 & 0.163249 \\
\hline 79 & 0.0720 & 13.8820 & 0.208141 \\
\hline 80 & 0.0712 & 14.0470 & 0.165694 \\
\hline 81 & 0.0710 & 14.0860 & 0.156214 \\
\hline 82 & 0.0705 & 14.1860 & 0.071075 \\
\hline 83 & 0.0699 & 14.3110 & 0.029681 \\
\hline 84 & 0.0696 & 14.3620 & 0.179891 \\
\hline 85 & 0.0684 & 14.6190 & 0.208418 \\
\hline 86 & 0.0677 & 14.7610 & 0.154371 \\
\hline 87 & 0.0672 & 14.8880 & 0.146310 \\
\hline
\end{tabular}

Continúa en la página siguiente. 


\begin{tabular}{|c|c|c|c|}
\hline Modo & Período (s) & Frecuencia (Hz) & Amortiguamiento por interacción suelo - estructura \\
\hline 88 & 0.0666 & 15.0180 & 0.019438 \\
\hline 89 & 0.0661 & 15.1350 & 0.054101 \\
\hline 90 & 0.0655 & 15.2780 & 0.152703 \\
\hline 91 & 0.0649 & 15.4060 & 0.147853 \\
\hline 92 & 0.0639 & 15.6480 & 0.054281 \\
\hline 93 & 0.0638 & 15.6770 & 0.182475 \\
\hline 94 & 0.0633 & 15.7880 & 0.046187 \\
\hline 95 & 0.0628 & 15.9310 & 0.008358 \\
\hline 96 & 0.0617 & 16.2050 & 0.210775 \\
\hline 97 & 0.0610 & 16.3950 & 0.183403 \\
\hline 98 & 0.0607 & 16.4640 & 0.206507 \\
\hline 99 & 0.0599 & 16.6980 & 0.057641 \\
\hline 100 & 0.0594 & 16.8350 & 0.156631 \\
\hline 101 & 0.0593 & 16.8730 & 0.003605 \\
\hline 102 & 0.0583 & 17.1410 & 0.008247 \\
\hline 103 & 0.0571 & 17.5190 & 0.176422 \\
\hline 104 & 0.0569 & 17.5730 & 0.197877 \\
\hline 105 & 0.0565 & 17.6890 & 0.004903 \\
\hline 106 & 0.0561 & 17.8160 & 0.175275 \\
\hline 107 & 0.0561 & 17.8350 & 0.025154 \\
\hline 108 & 0.0558 & 17.9170 & 0.160236 \\
\hline 109 & 0.0553 & 18.0700 & 0.030722 \\
\hline 110 & 0.0542 & 18.4640 & 0.038457 \\
\hline 111 & 0.0538 & 18.5900 & 0.111397 \\
\hline 112 & 0.0526 & 19.0110 & 0.000716 \\
\hline 113 & 0.0511 & 19.5720 & 0.001241 \\
\hline 114 & 0.0507 & 19.7270 & 0.004776 \\
\hline 115 & 0.0505 & 19.7930 & 0.002459 \\
\hline 116 & 0.0501 & 19.9640 & 0.002799 \\
\hline 117 & 0.0467 & 21.4090 & 0.004097 \\
\hline 118 & 0.0466 & 21.4620 & 0.000904 \\
\hline 119 & 0.0460 & 21.7190 & 0.000882 \\
\hline 120 & 0.0452 & 22.1220 & 0.000374 \\
\hline 121 & 0.0443 & 22.5790 & 0.000188 \\
\hline 122 & 0.0432 & 23.1700 & 0.000773 \\
\hline 123 & 0.0429 & 23.3310 & 0.003711 \\
\hline 124 & 0.0426 & 23.4540 & 0.000755 \\
\hline
\end{tabular}

Continúa en la página siguiente. 


\begin{tabular}{|c|c|c|c|}
\hline Modo & Período (s) & Frecuencia $(\mathrm{Hz})$ & Amortiguamiento por interacción suelo - estructura \\
\hline 125 & 0.0413 & 24.2090 & 0.000398 \\
\hline 126 & 0.0403 & 24.8040 & 0.000364 \\
\hline 127 & 0.0400 & 25.0110 & 0.000549 \\
\hline 128 & 0.0397 & 25.1790 & 0.002727 \\
\hline 129 & 0.0391 & 25.5780 & 0.000104 \\
\hline 130 & 0.0388 & 25.7750 & 0.000217 \\
\hline 131 & 0.0384 & 26.0260 & 0.000240 \\
\hline 132 & 0.0374 & 26.7560 & 0.000268 \\
\hline 133 & 0.0373 & 26.8430 & 0.002225 \\
\hline 134 & 0.0367 & 27.2760 & 0.007634 \\
\hline 135 & 0.0363 & 27.5340 & 0.001008 \\
\hline 136 & 0.0359 & 27.8420 & 0.004841 \\
\hline 137 & 0.0358 & 27.8960 & 0.002358 \\
\hline 138 & 0.0354 & 28.2180 & 0.001319 \\
\hline 139 & 0.0352 & 28.4140 & 0.001966 \\
\hline 140 & 0.0350 & 28.5520 & 0.002919 \\
\hline 141 & 0.0350 & 28.5950 & 0.004564 \\
\hline 142 & 0.0348 & 28.7450 & 0.004432 \\
\hline 143 & 0.0346 & 28.8880 & 0.001778 \\
\hline 144 & 0.0345 & 28.9920 & 0.001562 \\
\hline 145 & 0.0343 & 29.1770 & 0.003382 \\
\hline 146 & 0.0339 & 29.5180 & 0.006568 \\
\hline 147 & 0.0336 & 29.7980 & 0.006856 \\
\hline 148 & 0.0335 & 29.8930 & 0.009586 \\
\hline
\end{tabular}

Cuadro 4.19: Valores de período, frecuencia y amortiguamiento para $E_{p} / G_{s}=5000$. Pérgola de Bobadilla II

$E_{p} / G_{s}=5000$. Resultados de aceleración

En la tabla 4.20 se puede observar toda la información relativa al punto de aceleración máxima.

\begin{tabular}{|c|c|}
\hline Máximo valor de aceleración & $3.804 \mathrm{~m} / \mathrm{s}^{2}$ \\
\hline Punto & 1 \\
\hline Tren & 2 \\
\hline Velocidad & $240 \mathrm{~km} / \mathrm{h}$ \\
\hline
\end{tabular}

Cuadro 4.20: Máxima aceleración para $E_{p} / G_{s}=5000$. Pérgola de Bobadilla II 
En la figura 4.28 se puede observar la envolvente de aceleración en el punto en el que se alcanza su valor máximo.

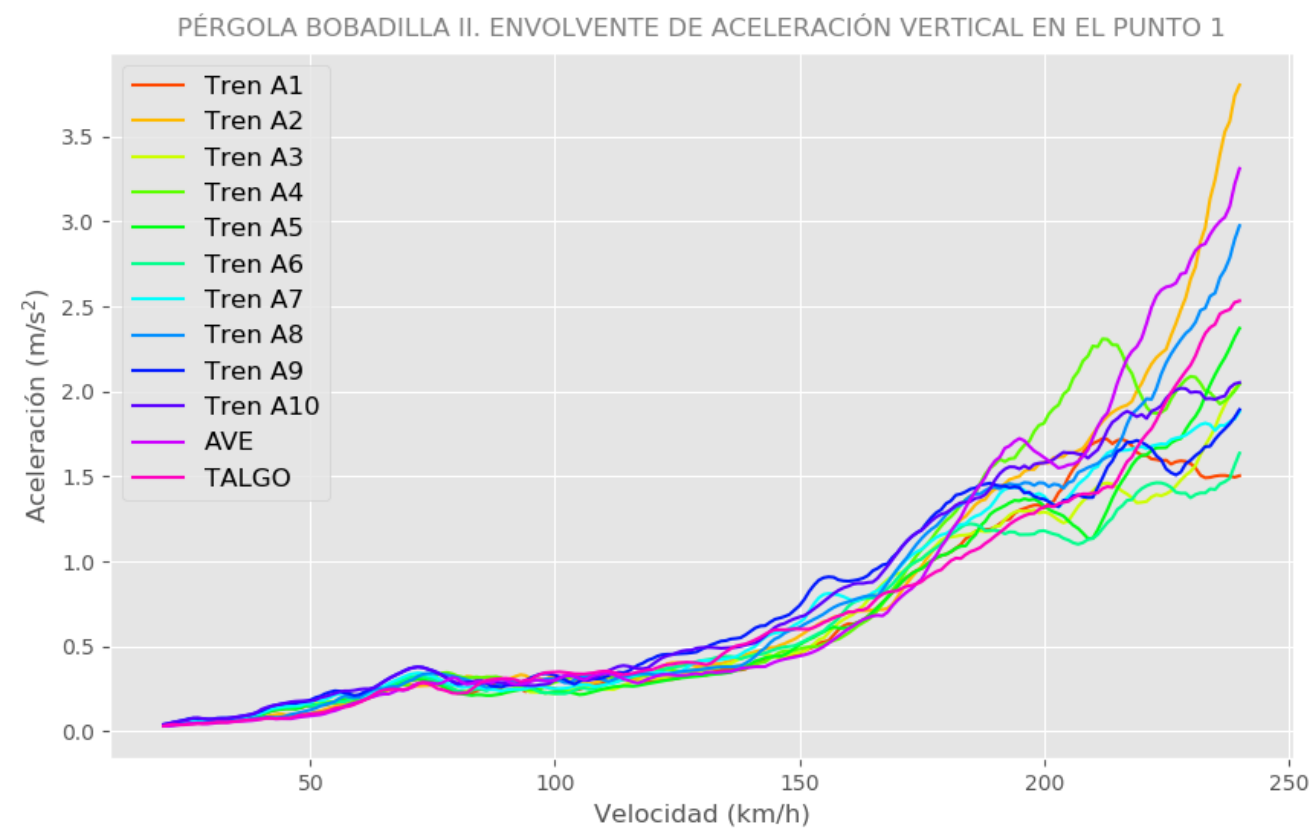

Figura 4.28: Envolvente de aceleración en el punto 1 para $E_{p} / G_{s}=5000$. Pérgola de Bobadilla II

$E_{p} / G_{s}=5000$. Resultados de desplazamiento

En la tabla 4.21 se puede observar toda la información relativa al punto de desplazamiento máximo.

\begin{tabular}{|c|c|}
\hline Máximo valor de desplazamiento & $2.908 \mathrm{~mm}$ \\
\hline Punto & 11 \\
\hline Tren & 9 \\
\hline Velocidad & $235 \mathrm{~km} / \mathrm{h}$ \\
\hline
\end{tabular}

Cuadro 4.21: Máximo desplazamiento para $E_{p} / G_{s}=5000$. Pérgola de Bobadilla II

En la figura 4.29 se puede observar la envolvente de desplazamiento en el punto en el que se alcanza su valor máximo. 


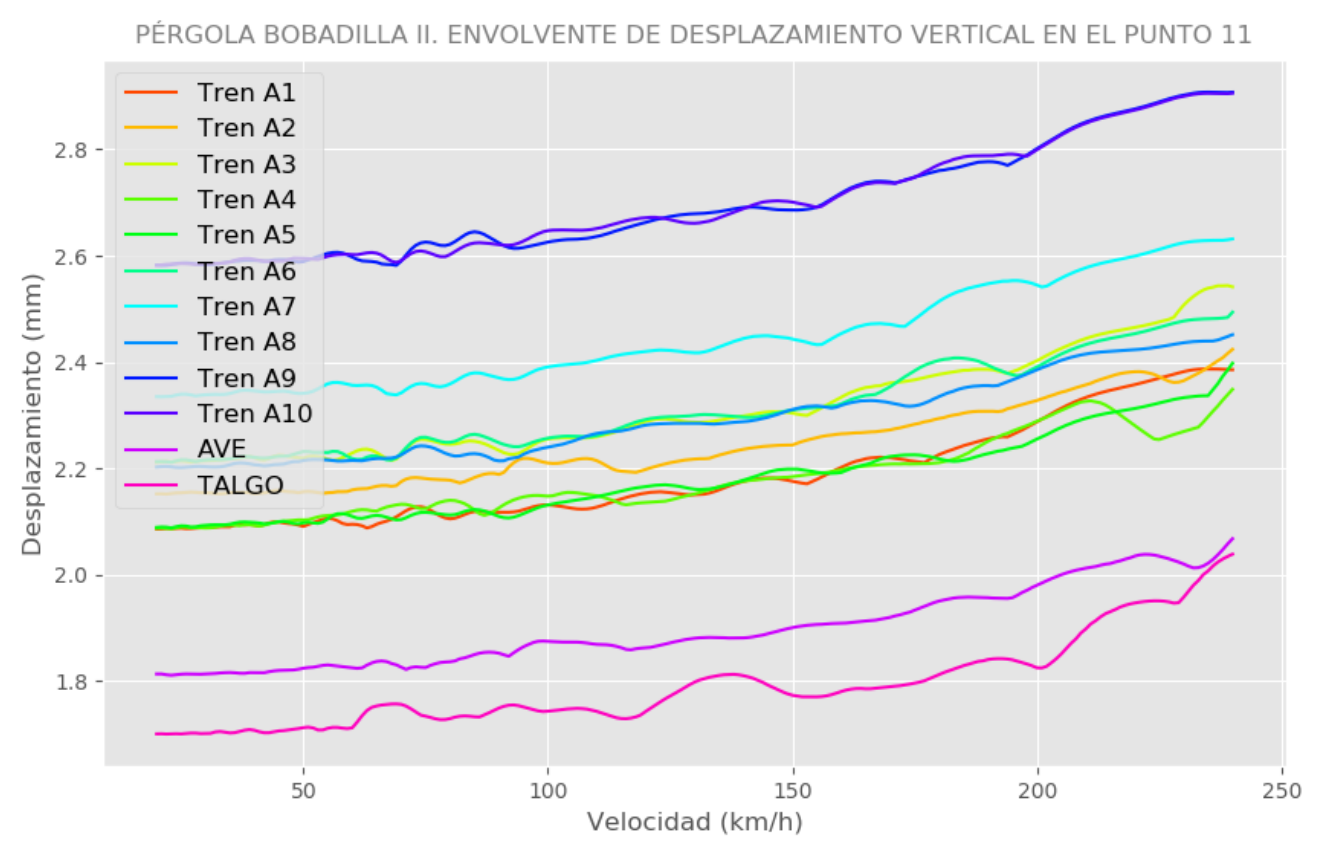

Figura 4.29: Envolvente de desplazamiento en el punto 11 para $E_{p} / G_{s}=5000$. Pérgola de Bobadilla II

Estructura con $E_{p} / G_{s}=10000$

$E_{p} / G_{s}=10000$. Análisis modal

Los modos de vibración con frecuencia inferior a $30 \mathrm{~Hz}$ y su amortiguamiento por la interacción suelo estructura se pueden observar en la tabla 4.22 .

\begin{tabular}{|c|c|c|c|}
\hline Modo & Período (s) & Frecuencia $(\mathrm{Hz})$ & Amortiguamiento por interacción suelo - estructura \\
\hline \hline 1 & 0.1876 & 5.3292 & 0.074878 \\
\hline 2 & 0.1845 & 5.4190 & 0.077855 \\
\hline 3 & 0.1768 & 5.6554 & 0.079828 \\
\hline 4 & 0.1687 & 5.9288 & 0.076061 \\
\hline 5 & 0.1663 & 6.0144 & 0.000537 \\
\hline 6 & 0.1642 & 6.0901 & 0.000793 \\
\hline 7 & 0.1598 & 6.2593 & 0.075366 \\
\hline 8 & 0.1546 & 6.4688 & 0.117421 \\
\hline 9 & 0.1534 & 6.5182 & 0.122753 \\
\hline 10 & 0.1491 & 6.7077 & 0.090445 \\
\hline 11 & 0.1433 & 6.9787 & 0.087089 \\
\hline 12 & 0.1405 & 7.1199 & 0.038546 \\
\hline 13 & 0.1388 & 7.2022 & 0.027332 \\
\hline
\end{tabular}

Continúa en la página siguiente. 


\begin{tabular}{|c|c|c|c|}
\hline Modo & Período (s) & Frecuencia $(\mathrm{Hz})$ & Amortiguamiento por interacción suelo - estructura \\
\hline 14 & 0.1370 & 7.3008 & 0.090469 \\
\hline 15 & 0.1354 & 7.3855 & 0.213935 \\
\hline 16 & 0.1349 & 7.4106 & 0.208634 \\
\hline 17 & 0.1343 & 7.4477 & 0.201350 \\
\hline 18 & 0.1336 & 7.4826 & 0.205885 \\
\hline 19 & 0.1330 & 7.5205 & 0.157502 \\
\hline 20 & 0.1322 & 7.5623 & 0.200943 \\
\hline 21 & 0.1295 & 7.7197 & 0.153718 \\
\hline 22 & 0.1289 & 7.7556 & 0.181455 \\
\hline 23 & 0.1259 & 7.9457 & 0.193393 \\
\hline 24 & 0.1256 & 7.9636 & 0.108997 \\
\hline 25 & 0.1232 & 8.1171 & 0.054833 \\
\hline 26 & 0.1228 & 8.1458 & 0.080032 \\
\hline 27 & 0.1214 & 8.2368 & 0.172465 \\
\hline 28 & 0.1210 & 8.2658 & 0.131128 \\
\hline 29 & 0.1204 & 8.3062 & 0.151545 \\
\hline 30 & 0.1194 & 8.3761 & 0.151283 \\
\hline 31 & 0.1183 & 8.4542 & 0.103356 \\
\hline 32 & 0.1176 & 8.5010 & 0.150034 \\
\hline 33 & 0.1168 & 8.5652 & 0.147037 \\
\hline 34 & 0.1161 & 8.6131 & 0.135077 \\
\hline 35 & 0.1127 & 8.8704 & 0.168346 \\
\hline 36 & 0.1121 & 8.9215 & 0.135077 \\
\hline 37 & 0.1116 & 8.9587 & 0.123906 \\
\hline 38 & 0.1105 & 9.0510 & 0.159061 \\
\hline 39 & 0.1099 & 9.1031 & 0.137138 \\
\hline 40 & 0.1092 & 9.1586 & 0.058848 \\
\hline 41 & 0.1084 & 9.2232 & 0.094408 \\
\hline 42 & 0.1082 & 9.2441 & 0.155905 \\
\hline 43 & 0.1073 & 9.3188 & 0.083322 \\
\hline 44 & 0.1070 & 9.3501 & 0.117093 \\
\hline 45 & 0.1056 & 9.4657 & 0.117763 \\
\hline 46 & 0.1052 & 9.5056 & 0.100782 \\
\hline 47 & 0.1050 & 9.5210 & 0.125849 \\
\hline 48 & 0.1040 & 9.6163 & 0.119081 \\
\hline 49 & 0.1027 & 9.7384 & 0.126953 \\
\hline 50 & 0.1018 & 9.8207 & 0.080908 \\
\hline
\end{tabular}

Continúa en la página siguiente. 


\begin{tabular}{|c|c|c|c|}
\hline Modo & Período (s) & Frecuencia $(\mathrm{Hz})$ & Amortiguamiento por interacción suelo - estructura \\
\hline 51 & 0.1009 & 9.9109 & 0.128451 \\
\hline 52 & 0.1003 & 9.9750 & 0.114582 \\
\hline 53 & 0.0990 & 10.1030 & 0.137182 \\
\hline 54 & 0.0988 & 10.1200 & 0.128275 \\
\hline 55 & 0.0981 & 10.1970 & 0.125855 \\
\hline 56 & 0.0966 & 10.3500 & 0.055103 \\
\hline 57 & 0.0965 & 10.3580 & 0.084873 \\
\hline 58 & 0.0953 & 10.4920 & 0.095460 \\
\hline 59 & 0.0944 & 10.5900 & 0.116275 \\
\hline 60 & 0.0938 & 10.6660 & 0.034779 \\
\hline 61 & 0.0932 & 10.7300 & 0.112732 \\
\hline 62 & 0.0925 & 10.8100 & 0.142754 \\
\hline 63 & 0.0917 & 10.9050 & 0.109180 \\
\hline 64 & 0.0909 & 11.0010 & 0.087275 \\
\hline 65 & 0.0898 & 11.1340 & 0.113533 \\
\hline 66 & 0.0889 & 11.2470 & 0.127946 \\
\hline 67 & 0.0883 & 11.3210 & 0.138945 \\
\hline 68 & 0.0878 & 11.3840 & 0.118337 \\
\hline 69 & 0.0878 & 11.3940 & 0.020079 \\
\hline 70 & 0.0863 & 11.5920 & 0.124945 \\
\hline 71 & 0.0858 & 11.6520 & 0.138794 \\
\hline 72 & 0.0857 & 11.6750 & 0.059012 \\
\hline 73 & 0.0850 & 11.7610 & 0.076724 \\
\hline 74 & 0.0834 & 11.9870 & 0.051825 \\
\hline 75 & 0.0829 & 12.0620 & 0.111587 \\
\hline 76 & 0.0823 & 12.1440 & 0.051187 \\
\hline 77 & 0.0815 & 12.2740 & 0.093259 \\
\hline 78 & 0.0801 & 12.4900 & 0.061777 \\
\hline 79 & 0.0794 & 12.5920 & 0.095834 \\
\hline 80 & 0.0786 & 12.7300 & 0.014225 \\
\hline 81 & 0.0781 & 12.8050 & 0.108855 \\
\hline 82 & 0.0768 & 13.0140 & 0.033587 \\
\hline 83 & 0.0748 & 13.3730 & 0.070412 \\
\hline 84 & 0.0744 & 13.4440 & 0.115727 \\
\hline 85 & 0.0739 & 13.5360 & 0.039251 \\
\hline 86 & 0.0724 & 13.8190 & 0.042520 \\
\hline 87 & 0.0721 & 13.8720 & 0.088531 \\
\hline
\end{tabular}

Continúa en la página siguiente. 


\begin{tabular}{|c|c|c|c|}
\hline Modo & Período (s) & Frecuencia $(\mathrm{Hz})$ & Amortiguamiento por interacción suelo - estructura \\
\hline 88 & 0.0709 & 14.0960 & 0.009648 \\
\hline 89 & 0.0701 & 14.2570 & 0.025427 \\
\hline 90 & 0.0698 & 14.3340 & 0.109903 \\
\hline 91 & 0.0685 & 14.5940 & 0.090730 \\
\hline 92 & 0.0682 & 14.6620 & 0.043779 \\
\hline 93 & 0.0681 & 14.6870 & 0.092832 \\
\hline 94 & 0.0667 & 14.9890 & 0.006000 \\
\hline 95 & 0.0661 & 15.1380 & 0.059778 \\
\hline 96 & 0.0659 & 15.1690 & 0.042742 \\
\hline 97 & 0.0647 & 15.4510 & 0.008695 \\
\hline 98 & 0.0635 & 15.7510 & 0.011128 \\
\hline 99 & 0.0631 & 15.8430 & 0.107149 \\
\hline 100 & 0.0629 & 15.8900 & 0.022674 \\
\hline 101 & 0.0626 & 15.9790 & 0.073977 \\
\hline 102 & 0.0621 & 16.1100 & 0.108408 \\
\hline 103 & 0.0610 & 16.3830 & 0.096048 \\
\hline 104 & 0.0603 & 16.5870 & 0.007892 \\
\hline 105 & 0.0594 & 16.8460 & 0.004093 \\
\hline 106 & 0.0584 & 17.1160 & 0.003212 \\
\hline 107 & 0.0580 & 17.2490 & 0.073618 \\
\hline 108 & 0.0566 & 17.6740 & 0.000736 \\
\hline 109 & 0.0561 & 17.8310 & 0.001930 \\
\hline 110 & 0.0556 & 17.9910 & 0.002869 \\
\hline 111 & 0.0541 & 18.4910 & 0.000472 \\
\hline 112 & 0.0526 & 19.0070 & 0.000186 \\
\hline 113 & 0.0511 & 19.5650 & 0.000361 \\
\hline 114 & 0.0508 & 19.6950 & 0.001566 \\
\hline 115 & 0.0506 & 19.7800 & 0.000447 \\
\hline 116 & 0.0501 & 19.9480 & 0.000646 \\
\hline 117 & 0.0468 & 21.3790 & 0.001549 \\
\hline 118 & 0.0466 & 21.4570 & 0.000219 \\
\hline 119 & 0.0461 & 21.7130 & 0.000271 \\
\hline 120 & 0.0452 & 22.1200 & 0.000136 \\
\hline 121 & 0.0443 & 22.5780 & 0.000071 \\
\hline 122 & 0.0432 & 23.1640 & 0.000295 \\
\hline 123 & 0.0429 & 23.3050 & 0.001388 \\
\hline 124 & 0.0426 & 23.4480 & 0.000276 \\
\hline
\end{tabular}

Continúa en la página siguiente. 


\begin{tabular}{|c|c|c|c|}
\hline Modo & Período (s) & Frecuencia $(\mathrm{Hz})$ & Amortiguamiento por interacción suelo - estructura \\
\hline 125 & 0.0413 & 24.2060 & 0.000151 \\
\hline 126 & 0.0403 & 24.8010 & 0.000149 \\
\hline 127 & 0.0400 & 25.0070 & 0.000246 \\
\hline 128 & 0.0397 & 25.1590 & 0.001053 \\
\hline 129 & 0.0391 & 25.5770 & 0.000042 \\
\hline 130 & 0.0388 & 25.7730 & 0.000091 \\
\hline 131 & 0.0384 & 26.0240 & 0.000102 \\
\hline 132 & 0.0374 & 26.7540 & 0.000126 \\
\hline 133 & 0.0373 & 26.8270 & 0.000902 \\
\hline 134 & 0.0367 & 27.2180 & 0.003249 \\
\hline 135 & 0.0363 & 27.5250 & 0.000542 \\
\hline 136 & 0.0360 & 27.8010 & 0.002428 \\
\hline 137 & 0.0359 & 27.8820 & 0.000627 \\
\hline 138 & 0.0355 & 28.2070 & 0.000603 \\
\hline 139 & 0.0352 & 28.3980 & 0.000983 \\
\hline 140 & 0.0351 & 28.5290 & 0.001245 \\
\hline 141 & 0.0350 & 28.5540 & 0.002610 \\
\hline 142 & 0.0348 & 28.7170 & 0.001234 \\
\hline 143 & 0.0346 & 28.8740 & 0.000749 \\
\hline 144 & 0.0345 & 28.9800 & 0.000665 \\
\hline 145 & 0.0343 & 29.1520 & 0.001394 \\
\hline 146 & 0.0339 & 29.4670 & 0.002850 \\
\hline 147 & 0.0336 & 29.7440 & 0.003183 \\
\hline 148 & 0.0335 & 29.8200 & 0.004023 \\
\hline
\end{tabular}

Cuadro 4.22: Valores de período, frecuencia y amortiguamiento para $E_{p} / G_{s}=10000$. Pérgola de Bobadilla II

$E_{p} / G_{s}=10000$. Resultados de aceleración

En la tabla 4.23 se puede observar toda la información relativa al punto de aceleración máxima.

\begin{tabular}{|c|c|}
\hline Máximo valor de aceleración & $4.318 \mathrm{~m} / \mathrm{s}^{2}$ \\
\hline Punto & 2 \\
\hline Tren & 2 \\
\hline Velocidad & $240 \mathrm{~km} / \mathrm{h}$ \\
\hline
\end{tabular}

Cuadro 4.23: Máxima aceleración para $E_{p} / G_{s}=10000$. Pérgola de Bobadilla II 
En la figura 4.30 se puede observar la envolvente de aceleración en el punto en el que se alcanza su valor máximo.

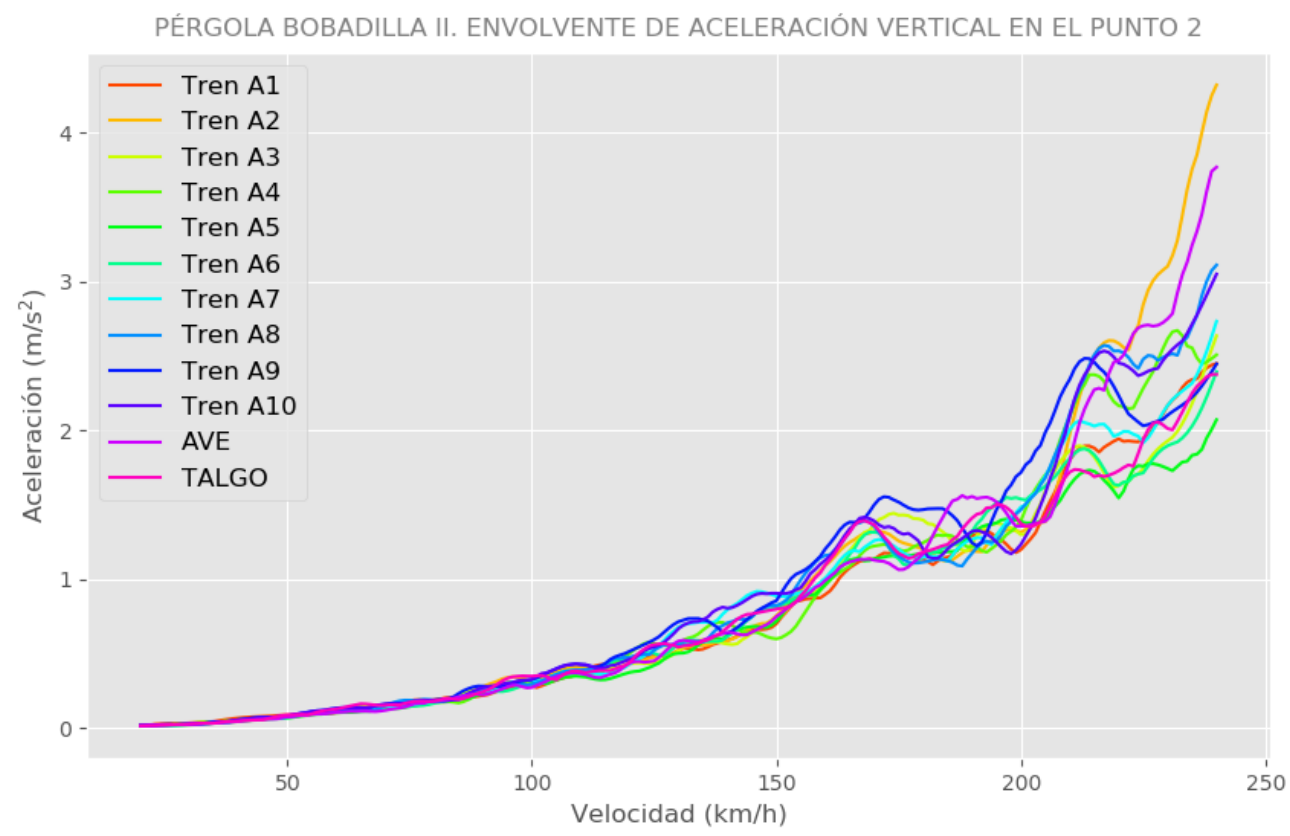

Figura 4.30: Envolvente de aceleración en el punto 2 para $E_{p} / G_{s}=10000$. Pérgola de Bobadilla II

$E_{p} / G_{s}=10000$. Resultados de desplazamiento

En la tabla 4.24 se puede observar toda la información relativa al punto de desplazamiento máximo.

\begin{tabular}{|c|c|}
\hline Máximo valor de desplazamiento & $3.173 \mathrm{~mm}$ \\
\hline Punto & 11 \\
\hline Tren & 10 \\
\hline Velocidad & $212 \mathrm{~km} / \mathrm{h}$ \\
\hline
\end{tabular}

Cuadro 4.24: Máximo desplazamiento para $E_{p} / G_{s}=10000$. Pérgola de Bobadilla II

En la figura 4.31 se puede observar la envolvente de desplazamiento en el punto en el que se alcanza su valor máximo. 


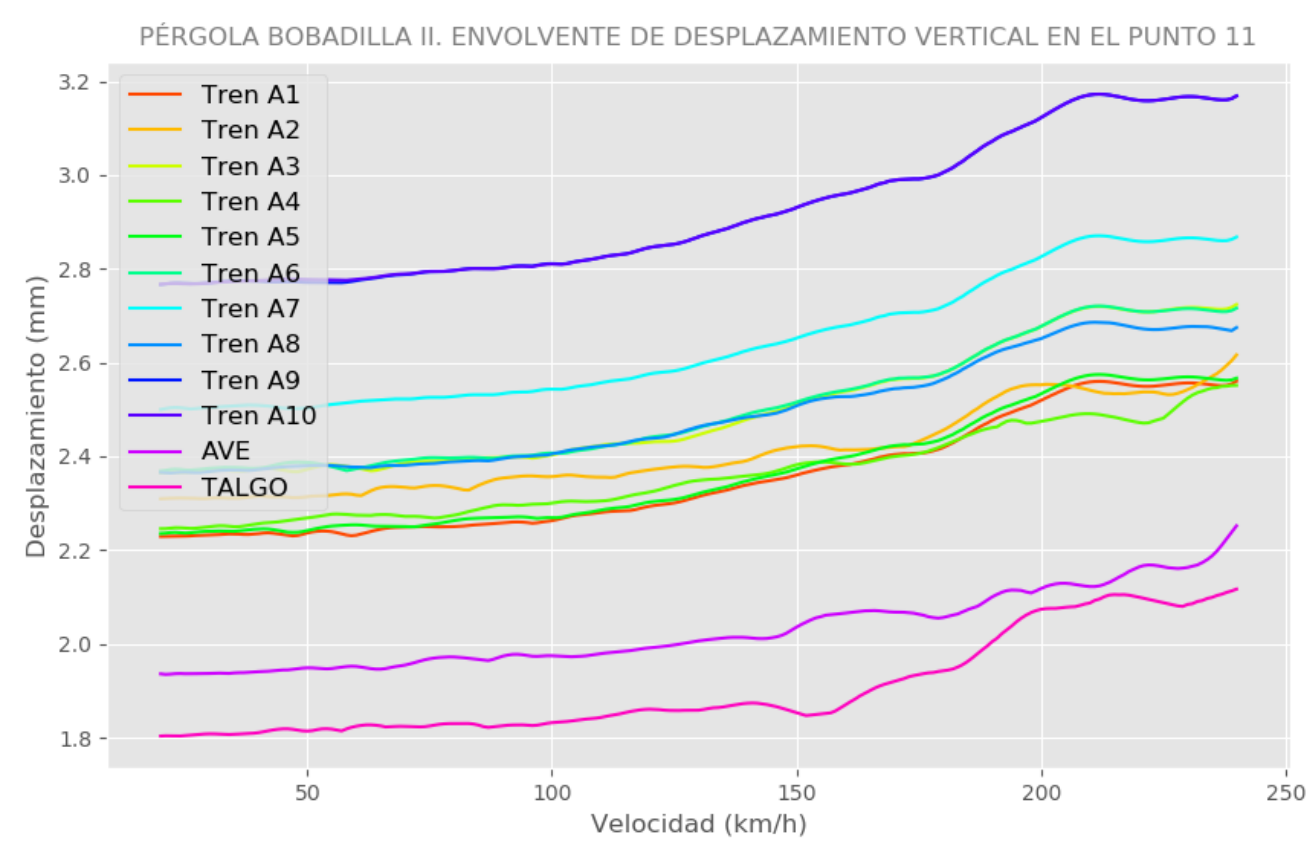

Figura 4.31: Envolvente de desplazamiento en el punto 11 para $E_{p} / G_{s}=10000$. Pérgola de Bobadilla II

\subsubsection{Resultados de la interacción suelo - estructura}

Resultados de aceleración

En la figura 4.32 se puede observar la variación de la aceleración máxima experimentada en la pérgola para cada una de las relaciones $E_{p} / G_{s}$ consideradas. 


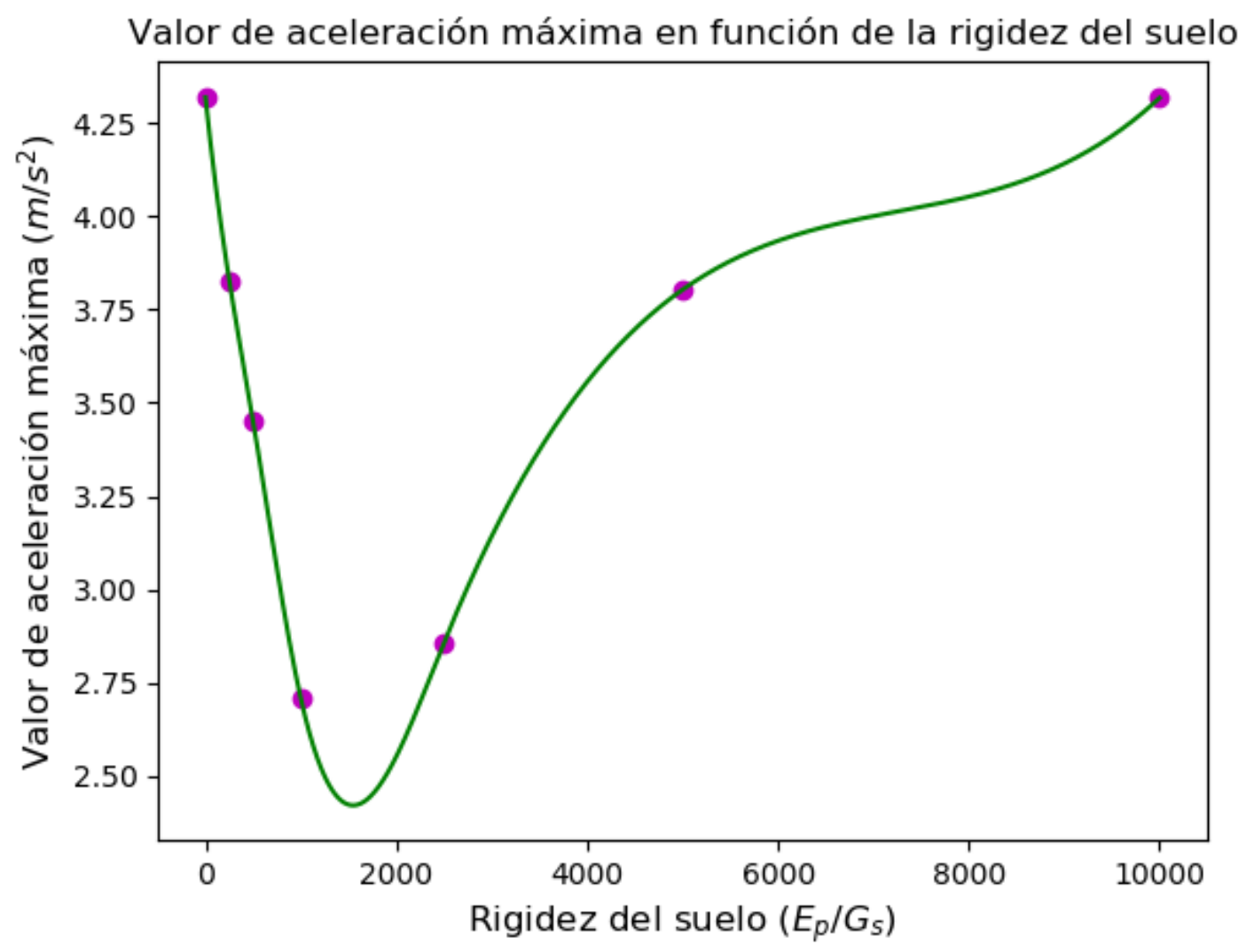

Figura 4.32: Aceleración máxima en función de $E_{p} / G_{s}$. Pérgola de Bobadilla II

Para poder entender los resultados reflejados en la gráfica hay que tener en cuenta lo siguiente:

1. El aumento de $E_{p} / G_{s}$ produce una disminución de la rigidez del suelo, lo que hace que las fecuencias de la estructura bajen, haciéndola más flexible. Este hecho aislado produciría un aumento de la aceleración máxima si no se contemplase el amortiguamiento producido por la interacción suelo - estructura.

2. Al ser el suelo menos rígido se producen unas oscilaciones más grandes en la estructura. Se activan los elementos muelle - amortiguador y hace que la disipación por interacción suelo - estructura sea mayor, a pesar de que a medida que disminuye la rigidez del suelo los valores de $k_{v}$ y $c_{v}$ son cada vez menores.

La gráfica 4.32 la podemos dividir en tres zonas claramente diferenciadas:

1. Zona entre $E_{p} / G_{s}=0$ y $E_{p} / G_{s}=1500 \mathrm{El}$ efecto de la disipación suelo estructura es mayor que el efecto de la pérdida de rigidez de la estructura, y a medida que aumenta $E_{p} / G_{s}$ la diferencia entre ambos es cada vez mayor. Esto produce una disminución de la aceleración máxima a medida que aumenta $E_{p} / G_{s}$. 
2. Zona entre $E_{p} / G_{s}=1500$ y $E_{p} / G_{s}=10000 \mathrm{El}$ efecto de la disipación suelo estructura es mayor que el efecto de la pérdida de rigidez de la estructura, y a medida que aumenta $E_{p} / G_{s}$ la diferencia entre ambos es cada vez menor. Esto produce un aumento de la aceleración máxima a medida que aumenta $E_{p} / G_{s}$, pero siempre con una valor menor al de la estructura modelada con terreno infinitamente rígido.

3. Zona superior a $E_{p} / G_{s}=10000 \mathrm{El}$ efecto de la disipación suelo - estructura y el efecto de la pérdida de rigidez de la estructura son prácticamente equivalentes, lo que hace que la aceleración máxima tenga aproximadamente el mismo valor que en la estructura modelada con terreno infinitamente rígido.

Resultados de desplazamiento

En la figura 4.33 se puede observar la variación de la aceleración máxima experimentada en la pérgola para cada una de las relaciones $E_{p} / G_{s}$ consideradas.

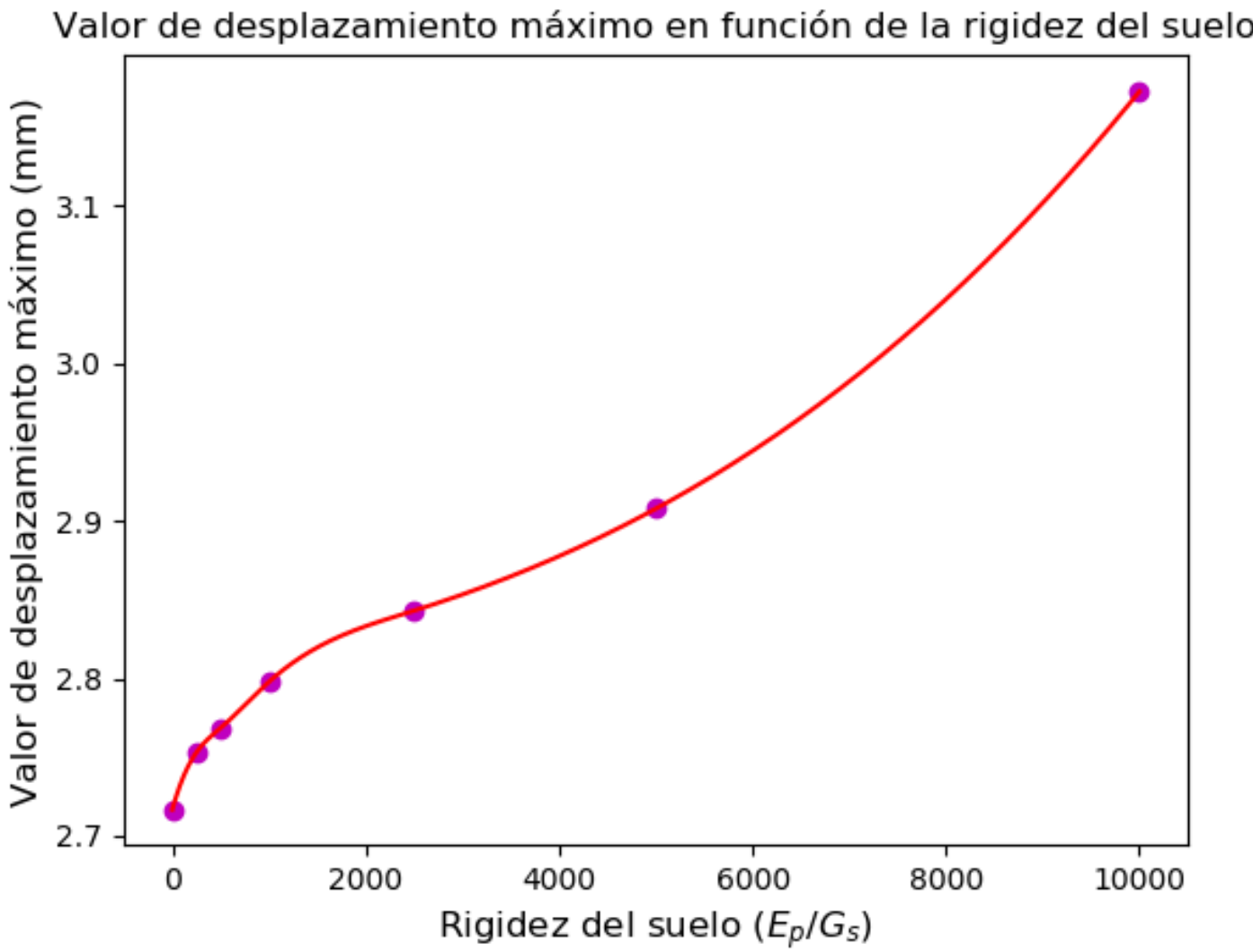

Figura 4.33: Desplazamiento máxima en función de $E_{p} / G_{s}$. Pérgola de Bobadilla II

En este caso. al convertirse la pérgola en una estructura más flexible se producen mayores oscilaciones, que hacen que aumente el valor de desplazamiento máximo experimentado bajo el paso de los trenes seleccionados. 


\section{CAPÍtulo 5}

\section{Conclusiones y aportaciones}

A continuación se describen las conclusiones obtenidas de la realización del presente documento:

1. El método semianalítico para resolver el problema de paso de cargas móviles presenta una serie de ventajas significativas con respecto a los métodos de integración por incrementos paso a paso, las cuales se sintetizan en los siguientes puntos:

- Importante disminución del tiempo de cálculo.

- Menor acumulación de errores por su cálculo de manera exacta en la variable temporal.

- Requerimiento de intervalos de tiempo no tan exigentes como los métodos paso a paso.

- Obtención de las respuestas dinámicas en los puntos de posproceso seleccionados por el usuario.

- Permite el empleo de intervalos de velocidad más pequeños que posibiliten la obtención de gráficas de respuesta de mayor precisión y calidad.

2. El modelizado de las pérgolas mediante la inclusión de la cimentación lleva acarreada una disminución de la frecuencia fundamental de la estructura.

3. La tasa de amortiguamiento geométrico asociado a cada uno de los modos de vibración depende fuertemente de la flexibilidad del suelo. A medida que el terreno se convierte en un elemento más flexible, la tasa de amortiguamiento modal por propagación de ondas a lo largo del mismo también aumenta, a pesar de que los valores de rigidez y amortiguamiento de los pilotes disminuyen.

4. El valor máximo de aceleración obtenido tras el paso de las cargas sobre la pérgola varía con la rigidez del terreno. Se produce un descenso del mismo hasta un cierto valor de rigidez del terreno y a continuación un ascenso hasta el valor inicial, de forma que un terreno infinitamente flexible presenta aproximadamente los mismos valores de aceleración que uno infinitamente rígido.

5. Mediante el presente trabajo se muestra una metodología sencilla y de fácil inclusión en el modelo de cálculo de una pérgola cimentada mediante pilotes por cualquier proyectista. La utilización de un modelo que incluya la interacción entre el suelo y la estructura permite obtener unos valores máximos de aceleración menores que si no se 
utilizara, lo que acarrea un abaratamiento de la estructura si es de nueva construcción, así como un aumento de la velocidad límite de paso si la pérgola estudiada ya está en servicio. 


\section{Apéndice A}

\section{Análisis dinámico de la pérgola de Bobadilla II}

En este apéndice se van a exponer los resultados dinámicos en todos los puntos de posproceso de aceleración (figura 4.8) y desplazamiento (figura 4.9).

\section{A.1. Resultado para estructura con apoyos}

\section{A.1.1. Resultados de aceleración}

ENVOLVENTE DE ACELERACIÓN VERTICAL EN EL PUNTO 1

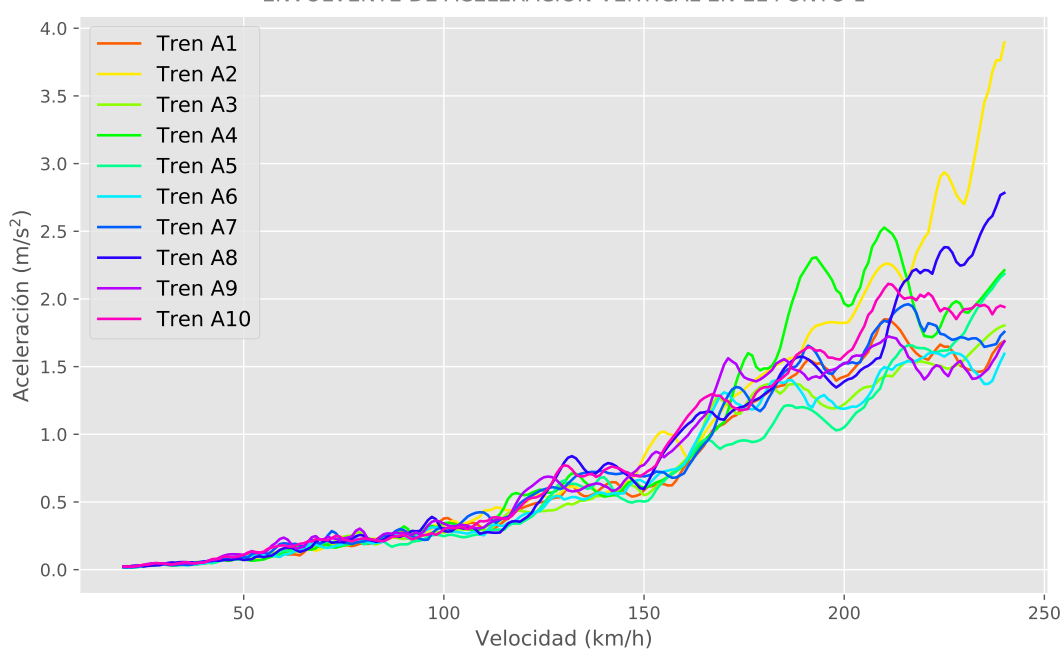


ENVOLVENTE DE ACELERACIÓN VERTICAL EN EL PUNTO 2

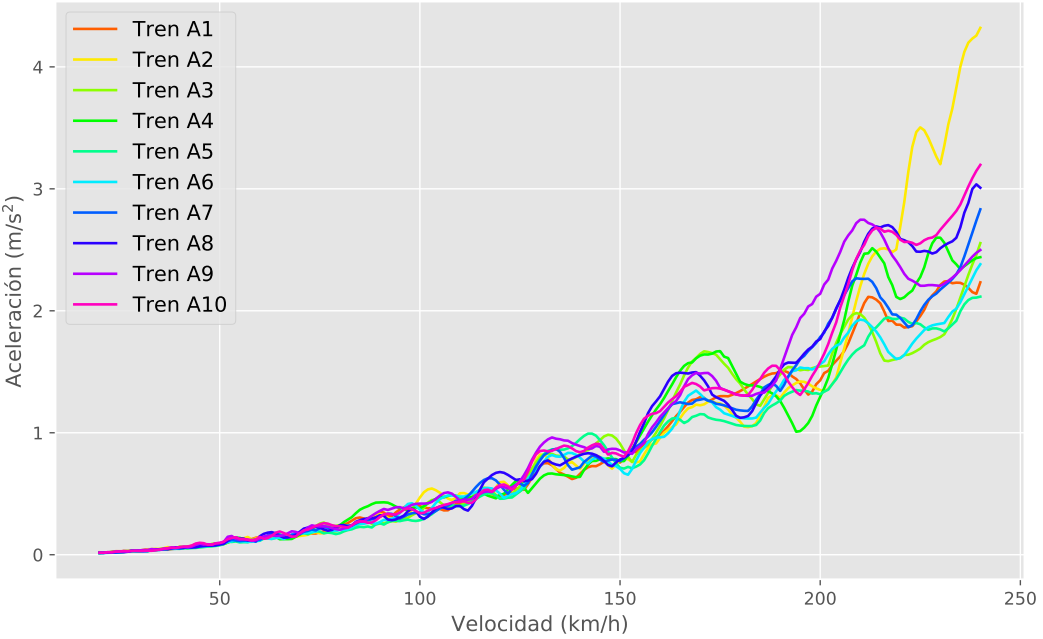

ENVOLVENTE DE ACELERACIÓN VERTICAL EN EL PUNTO 3

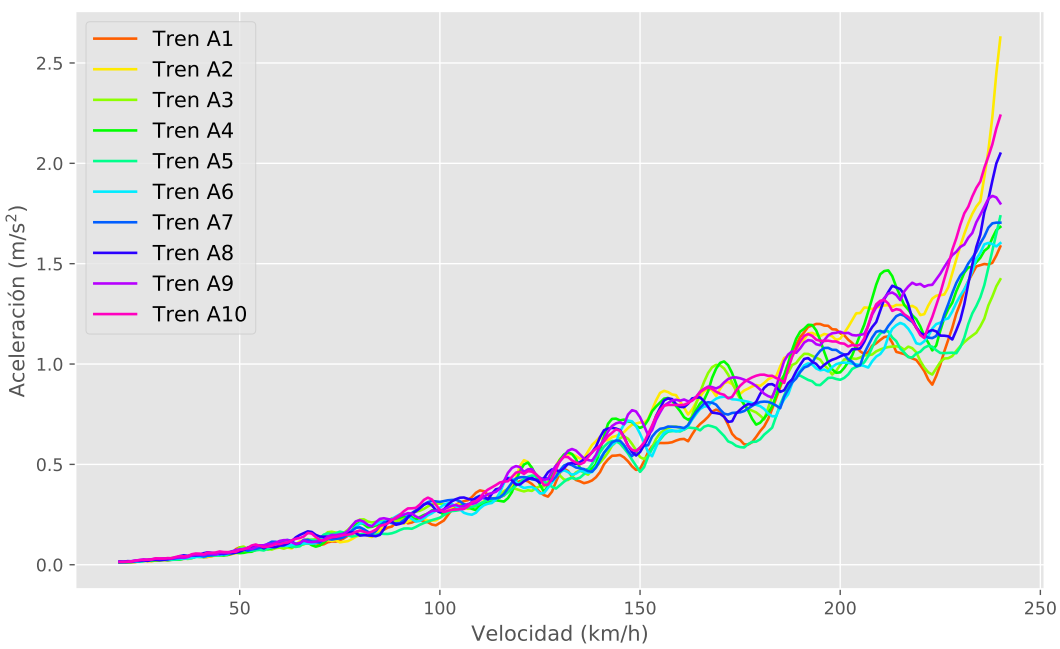




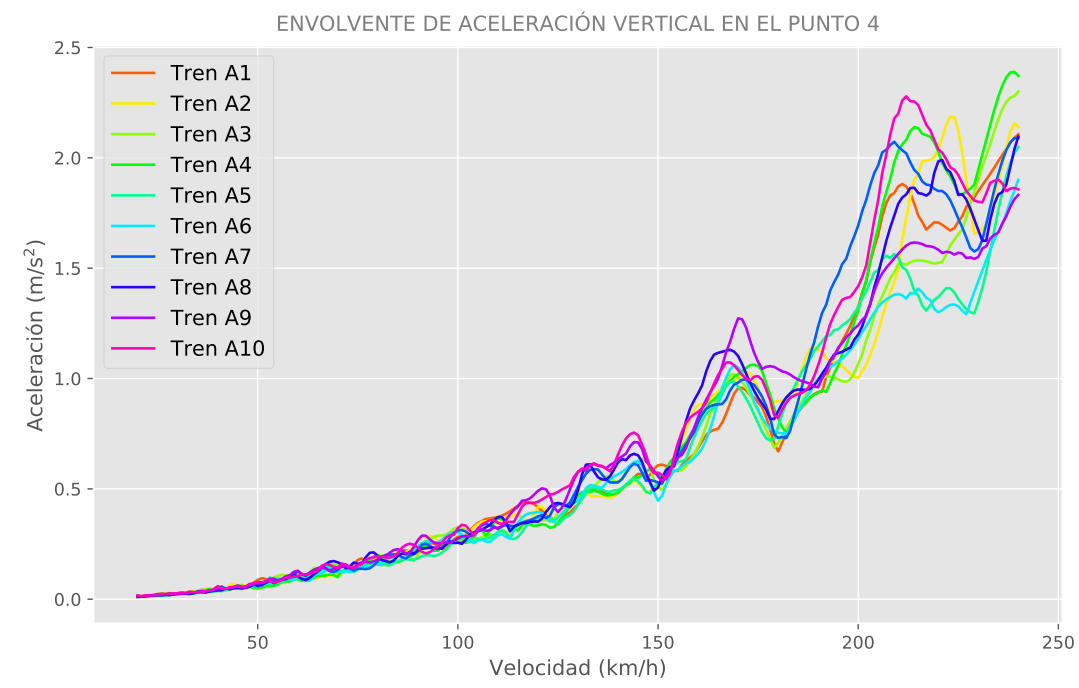

ENVOLVENTE DE ACELERACIÓN VERTICAL EN EL PUNTO 5

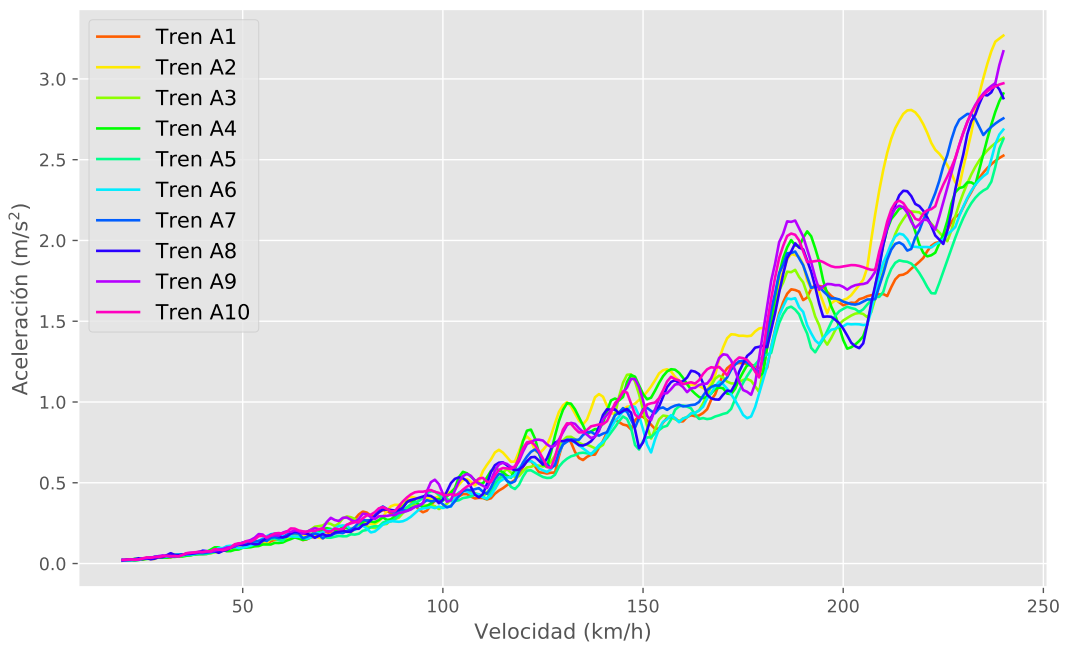


ENVOLVENTE DE ACELERACIÓN VERTICAL EN EL PUNTO 6

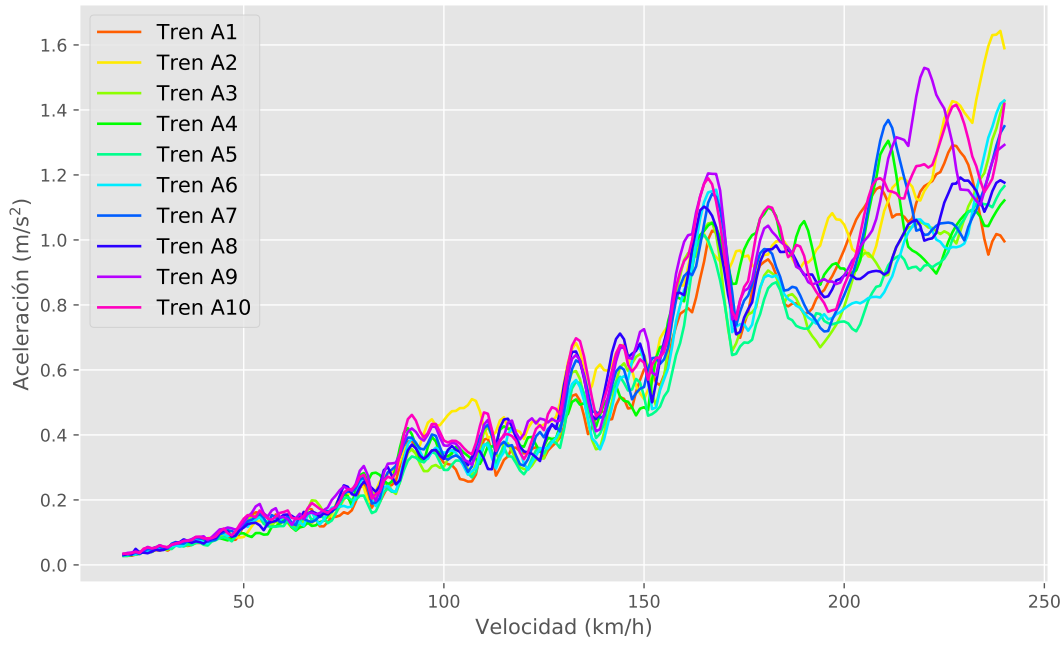

ENVOLVENTE DE ACELERACIÓN VERTICAL EN EL PUNTO 7

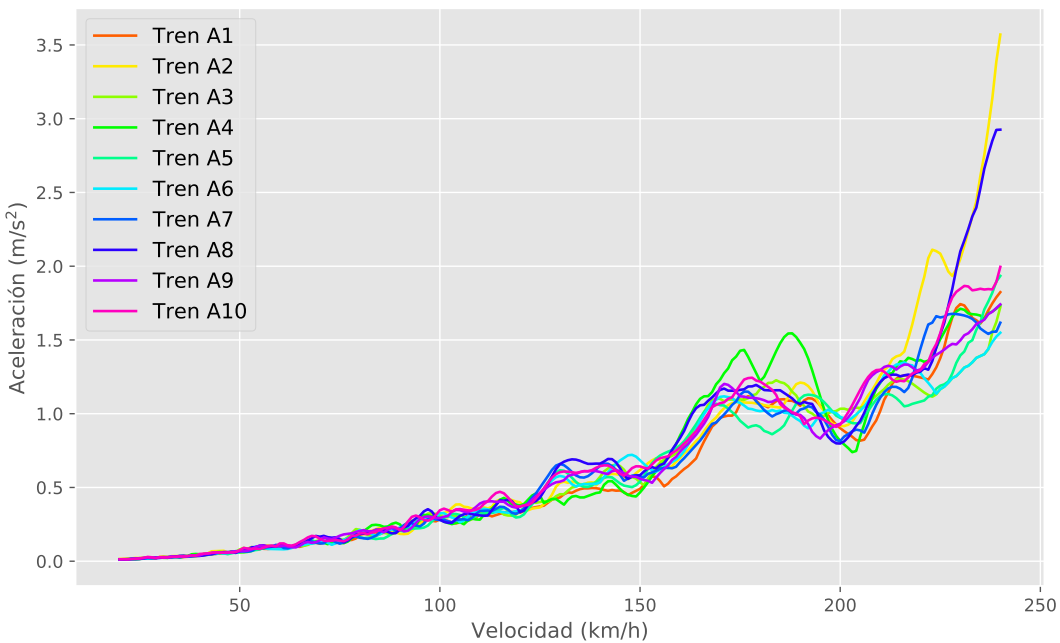


ENVOLVENTE DE ACELERACIÓN VERTICAL EN EL PUNTO 8

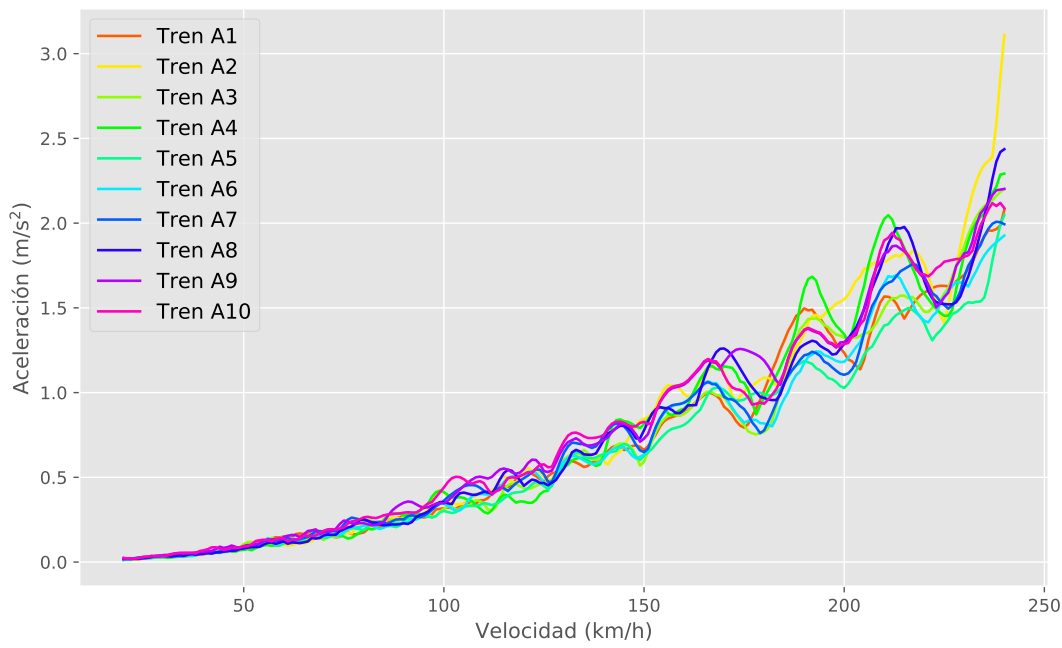

ENVOLVENTE DE ACELERACIÓN VERTICAL EN EL PUNTO 9

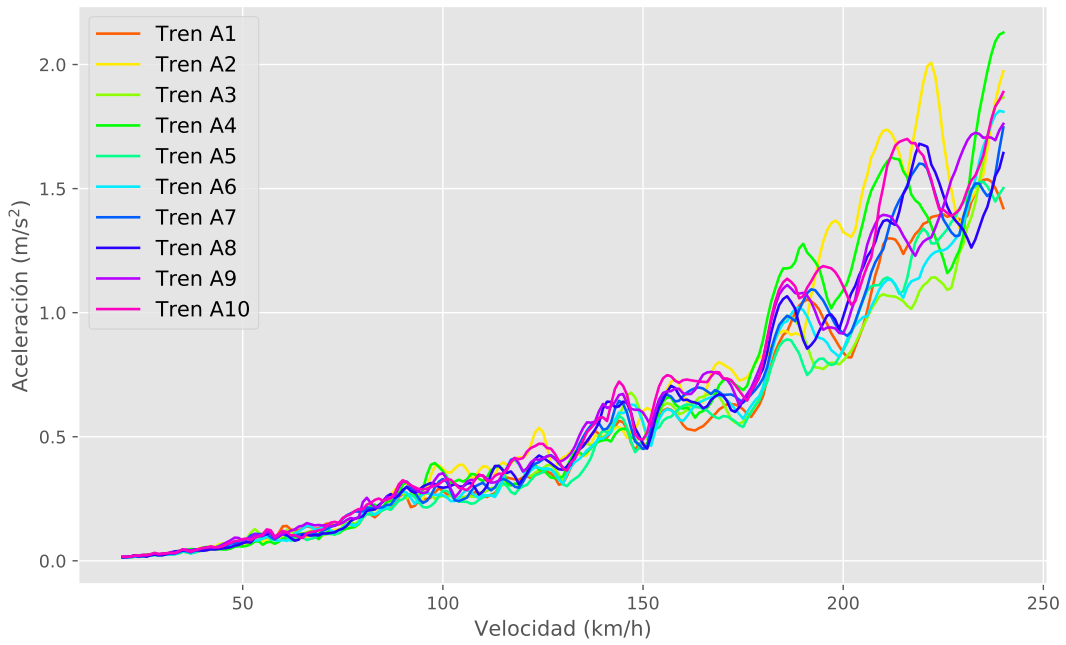




\section{A.1.2. Resultados de desplazamiento}
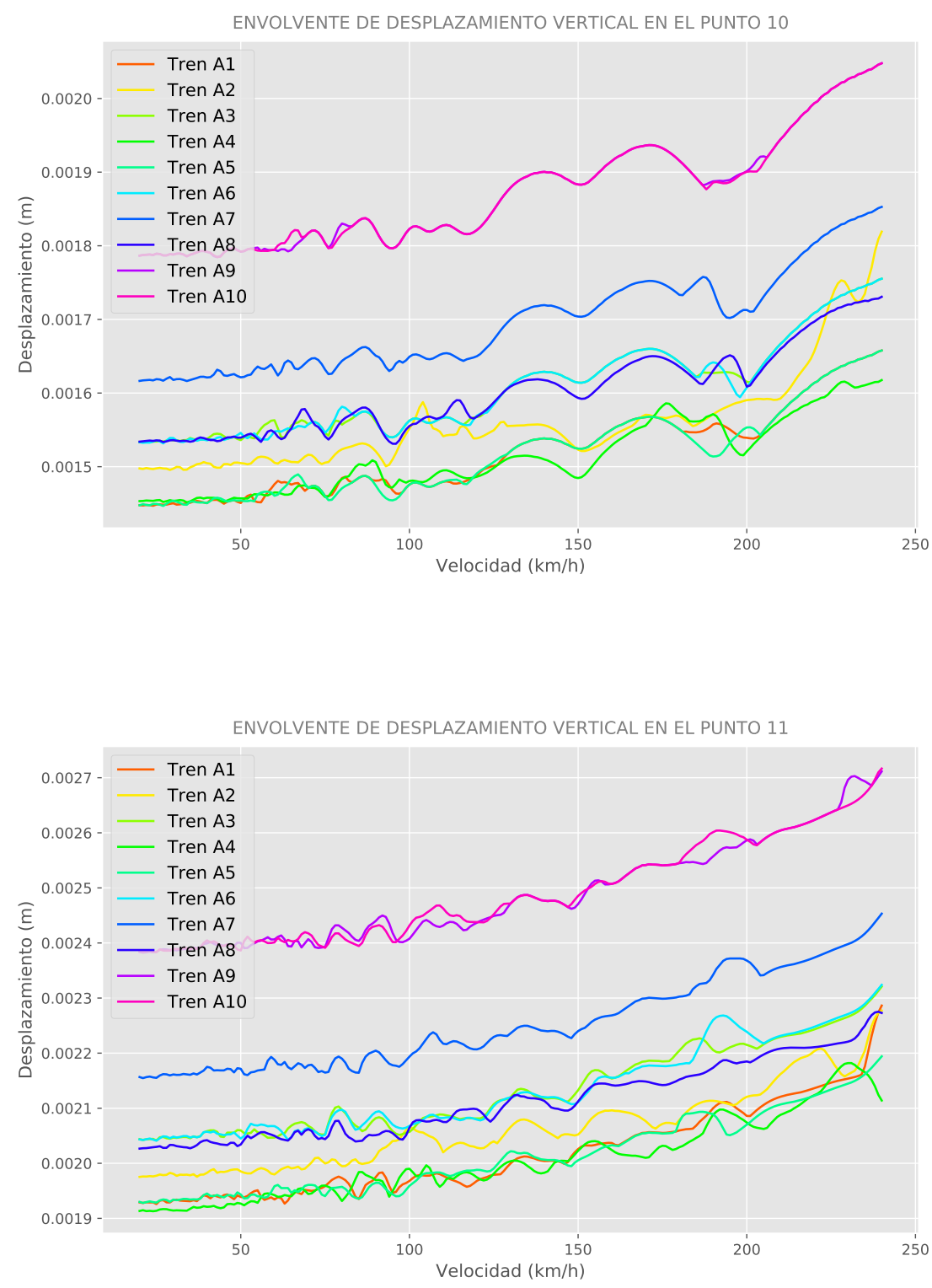


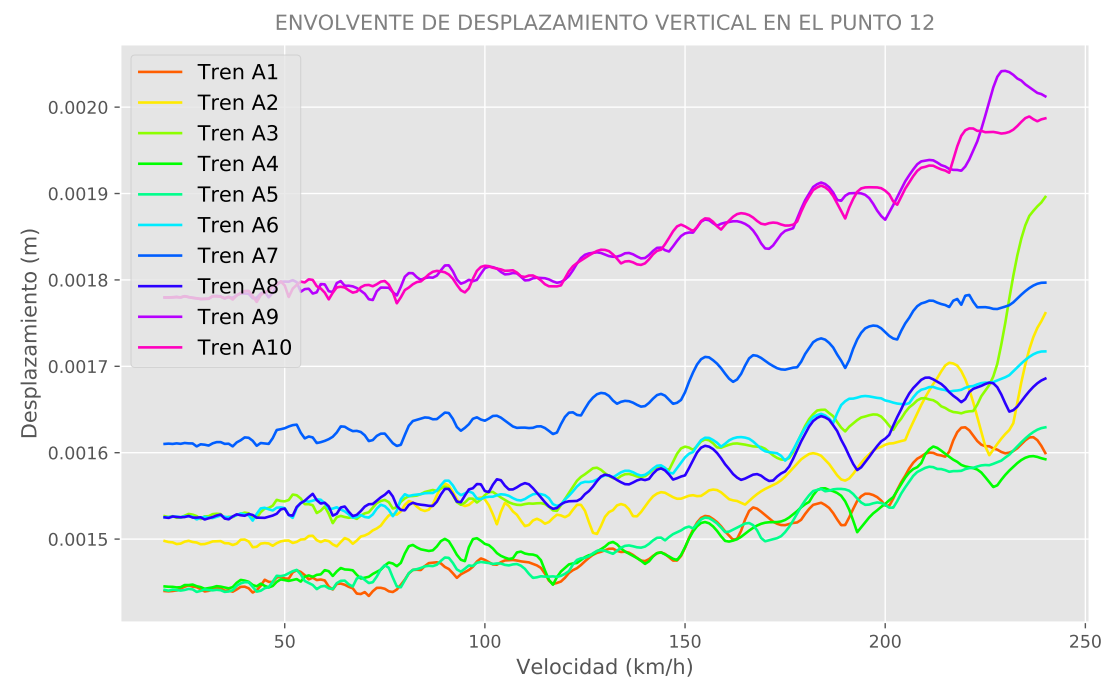

A.2. Resultados para $E_{p} / G_{s}=250$

\section{A.2.1. Resultados de aceleración}

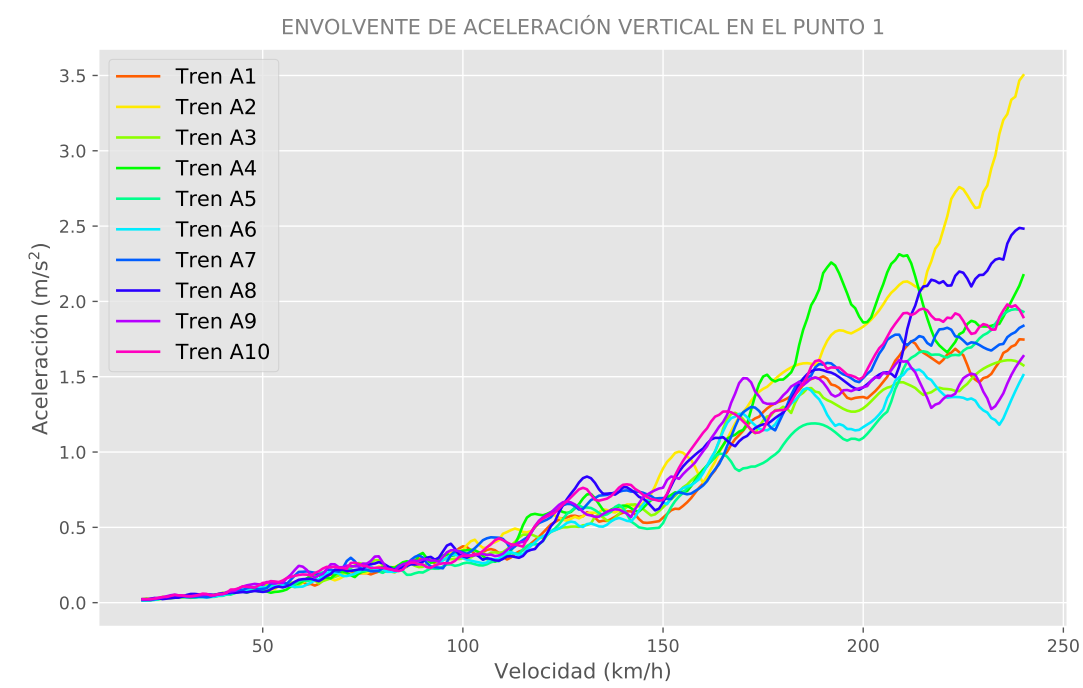




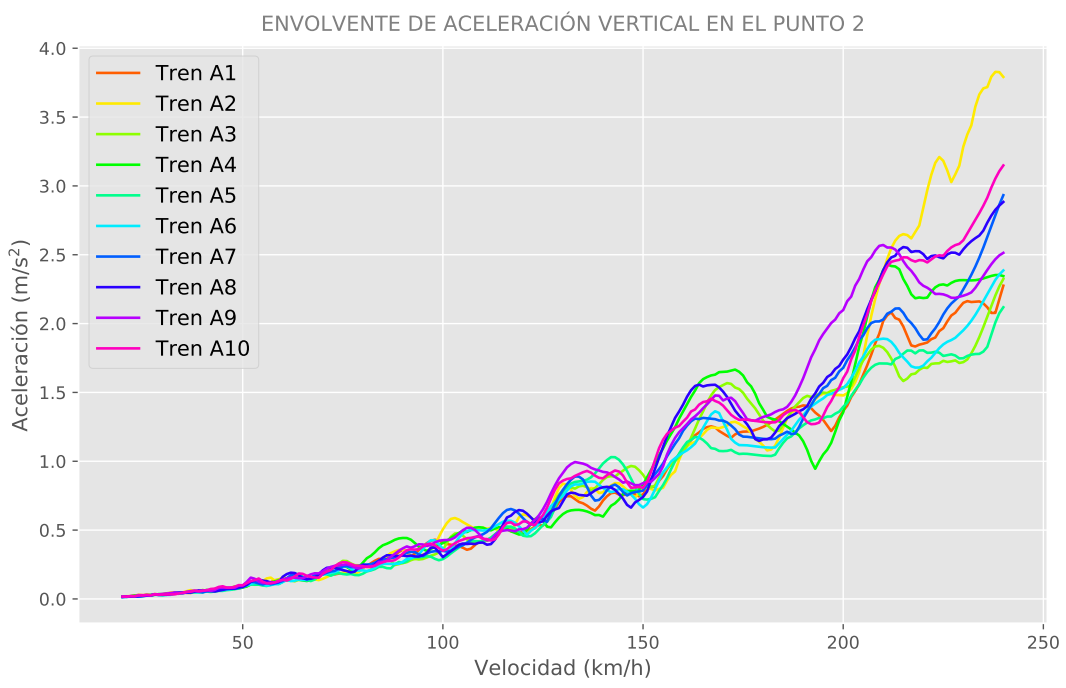

ENVOLVENTE DE ACELERACIÓN VERTICAL EN EL PUNTO 3

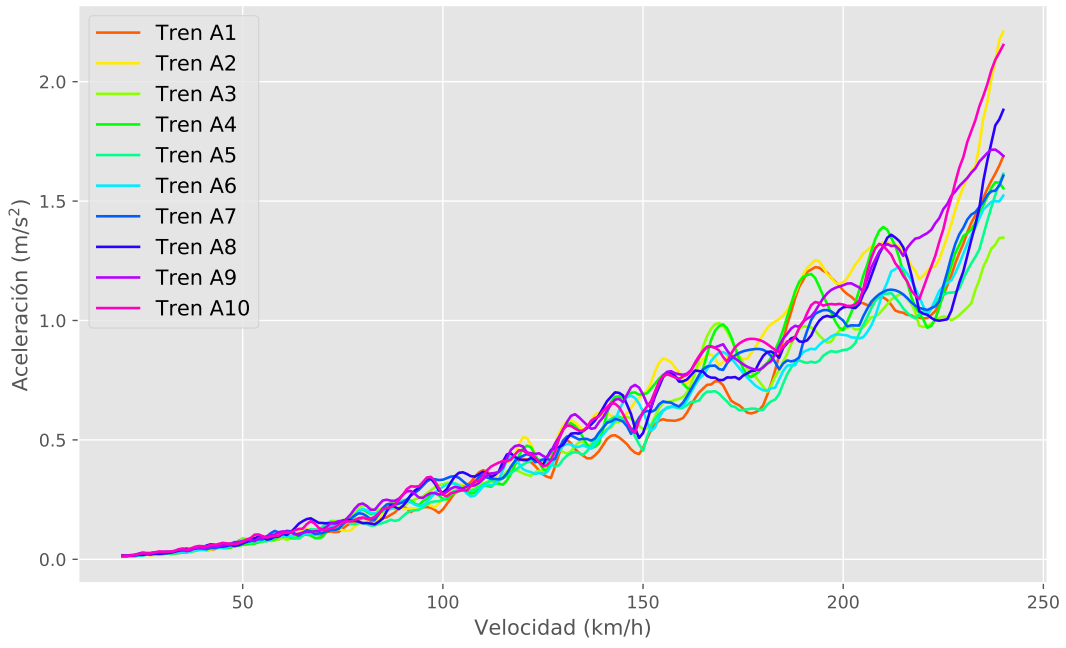


ENVOLVENTE DE ACELERACIÓN VERTICAL EN EL PUNTO 4

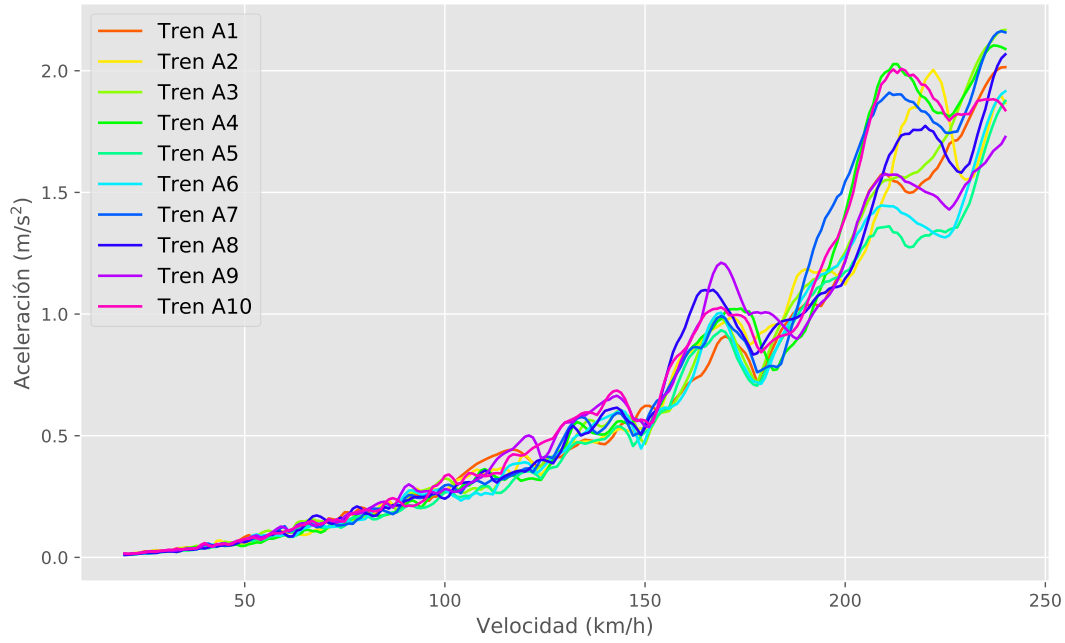

ENVOLVENTE DE ACELERACIÓN VERTICAL EN EL PUNTO 5

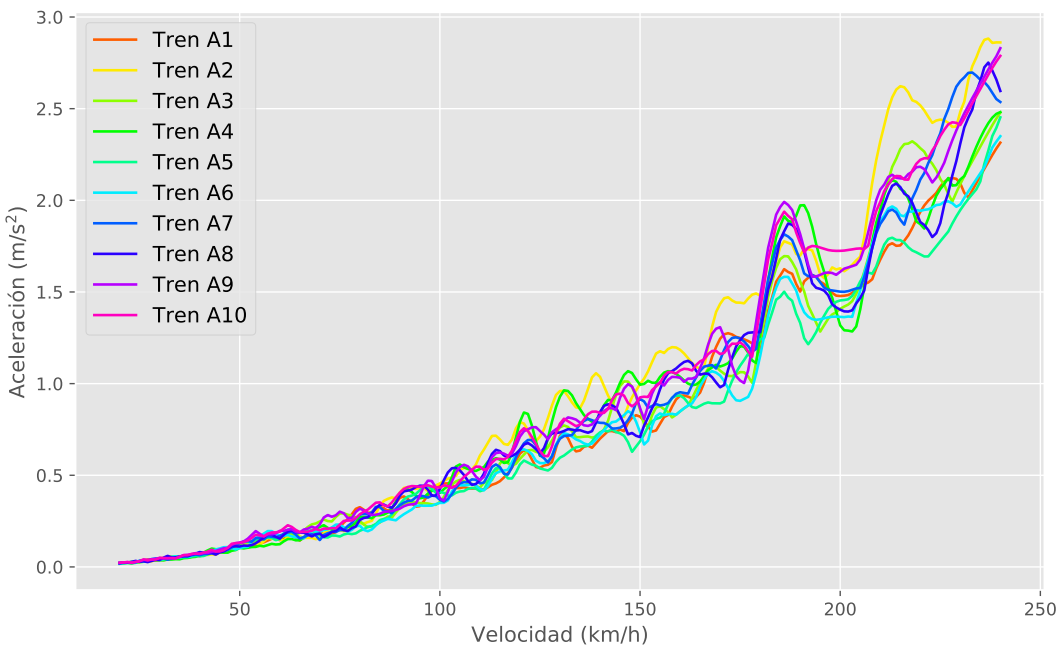


ENVOLVENTE DE ACELERACIÓN VERTICAL EN EL PUNTO 6

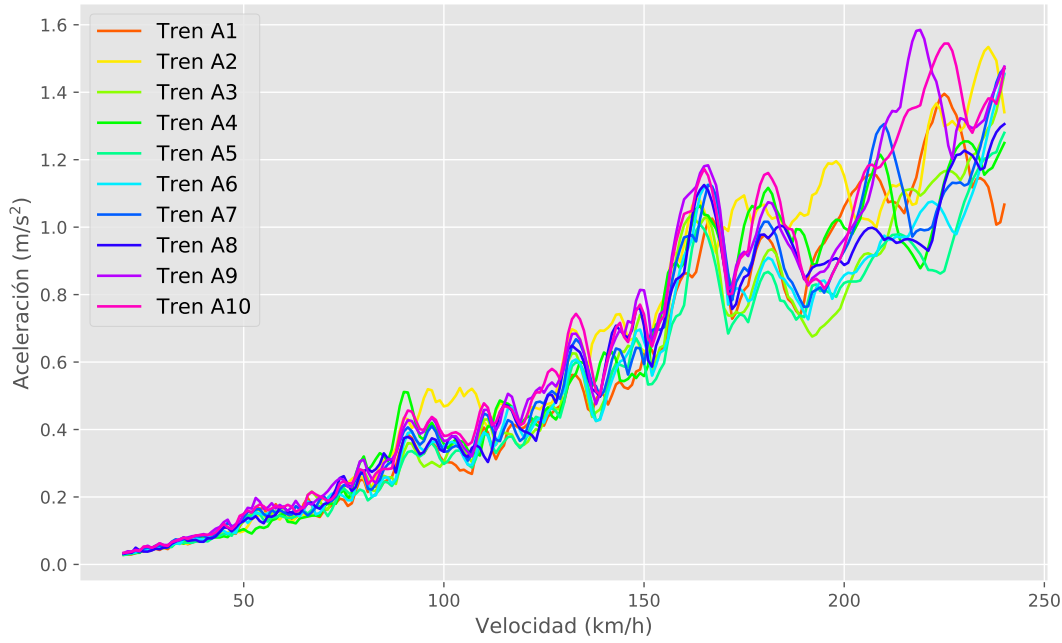

ENVOLVENTE DE ACELERACIÓN VERTICAL EN EL PUNTO 7

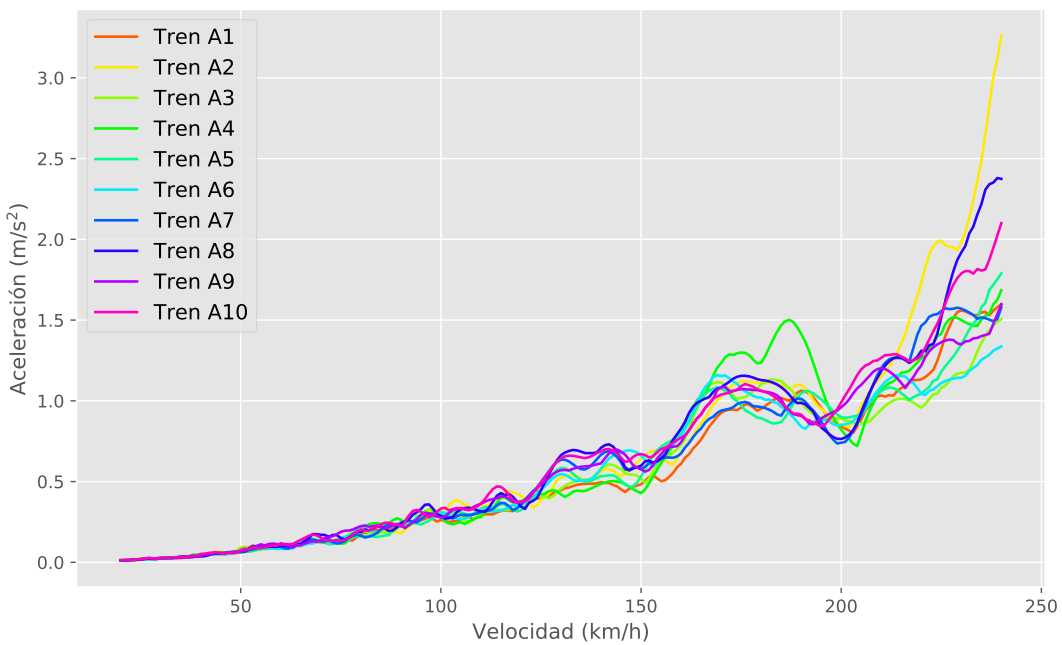


ENVOLVENTE DE ACELERACIÓN VERTICAL EN EL PUNTO 8

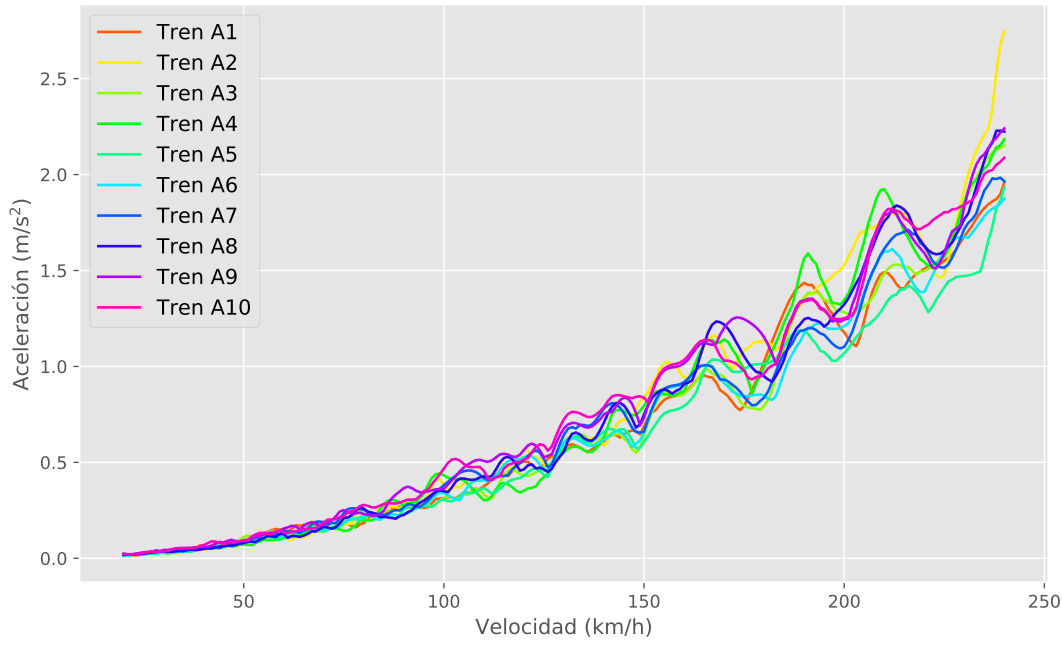

ENVOLVENTE DE ACELERACIÓN VERTICAL EN EL PUNTO 9

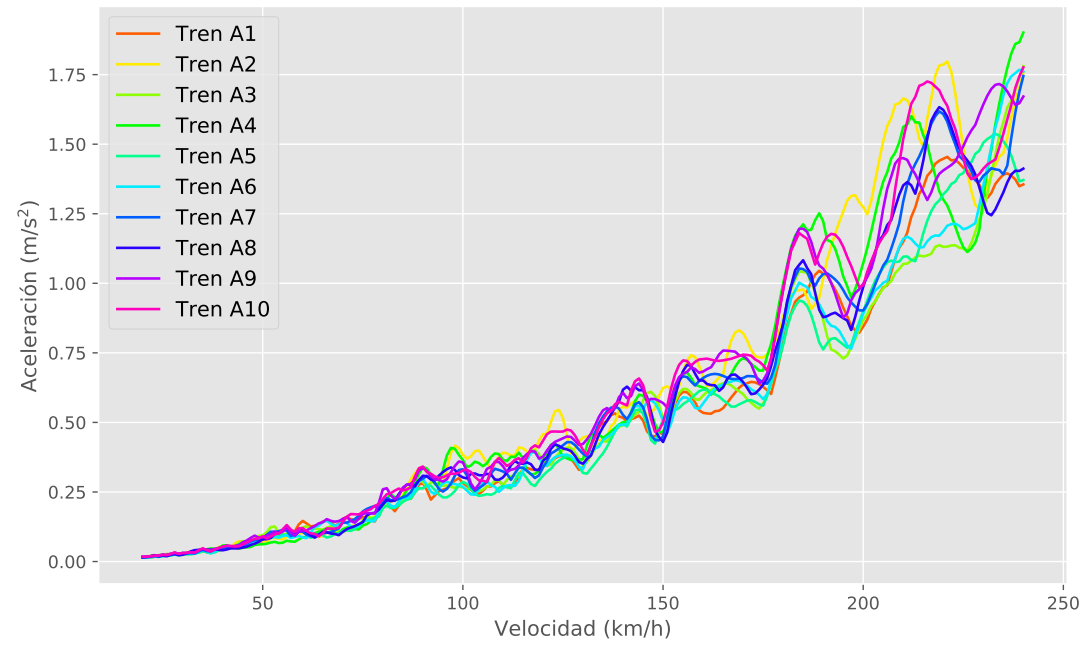




\section{A.2.2. Resultados de desplazamiento}
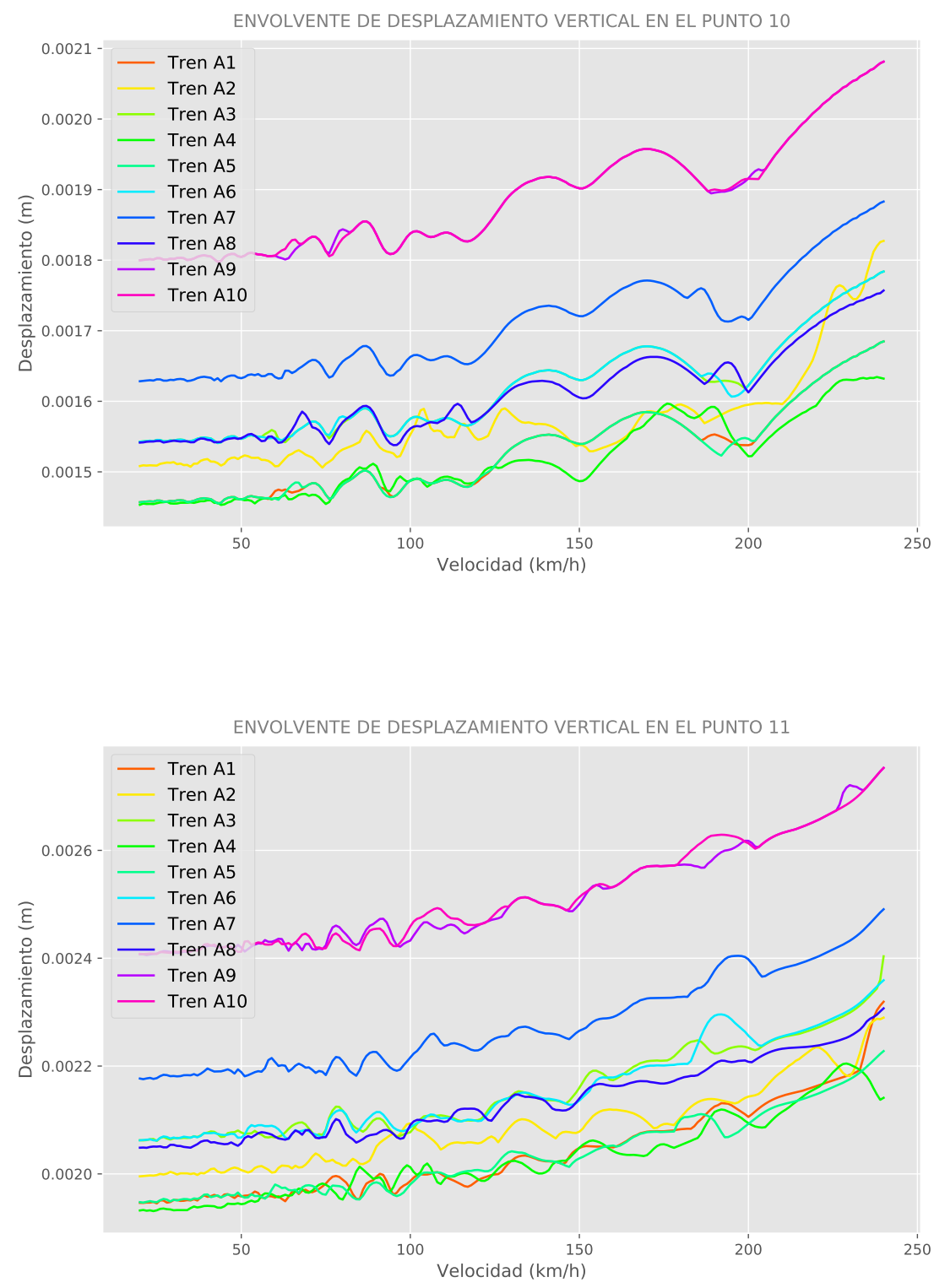
ENVOLVENTE DE DESPLAZAMIENTO VERTICAL EN EL PUNTO 12

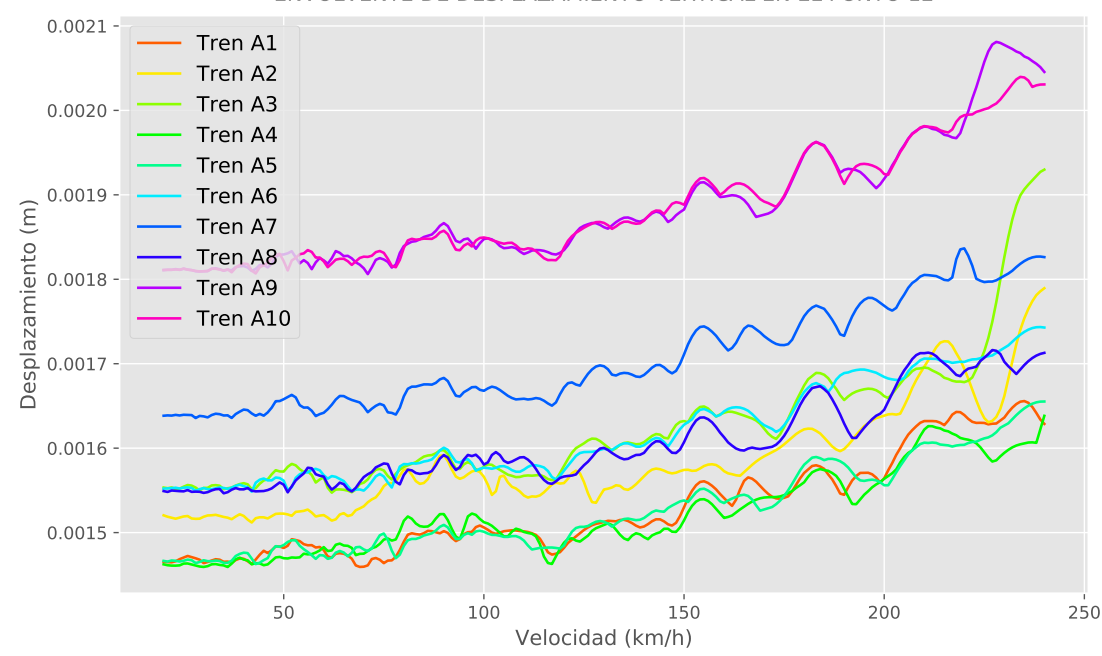

A.3. Resultados para $E_{p} / G_{s}=500$

\section{A.3.1. Resultados de aceleración}

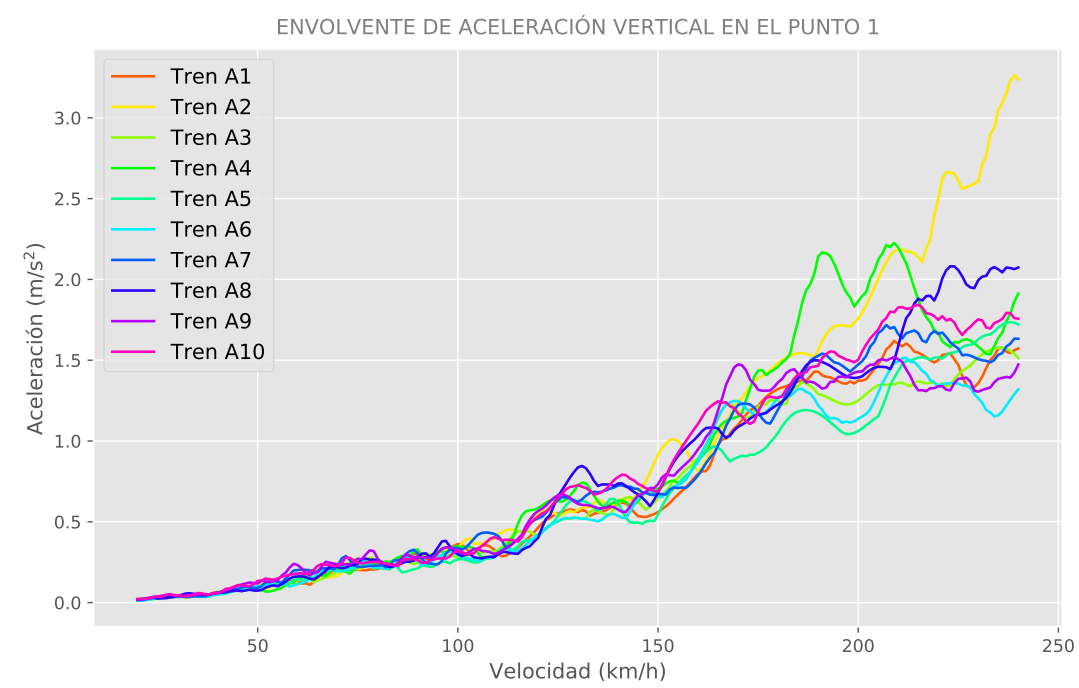


ENVOLVENTE DE ACELERACIÓN VERTICAL EN EL PUNTO 2

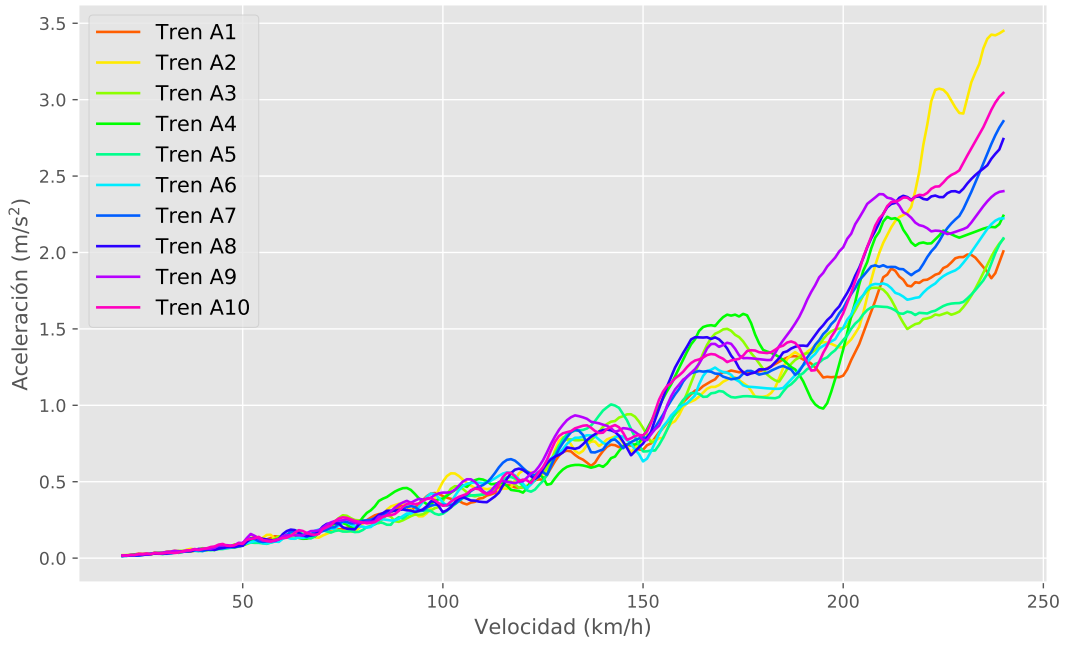

ENVOLVENTE DE ACELERACIÓN VERTICAL EN EL PUNTO 3

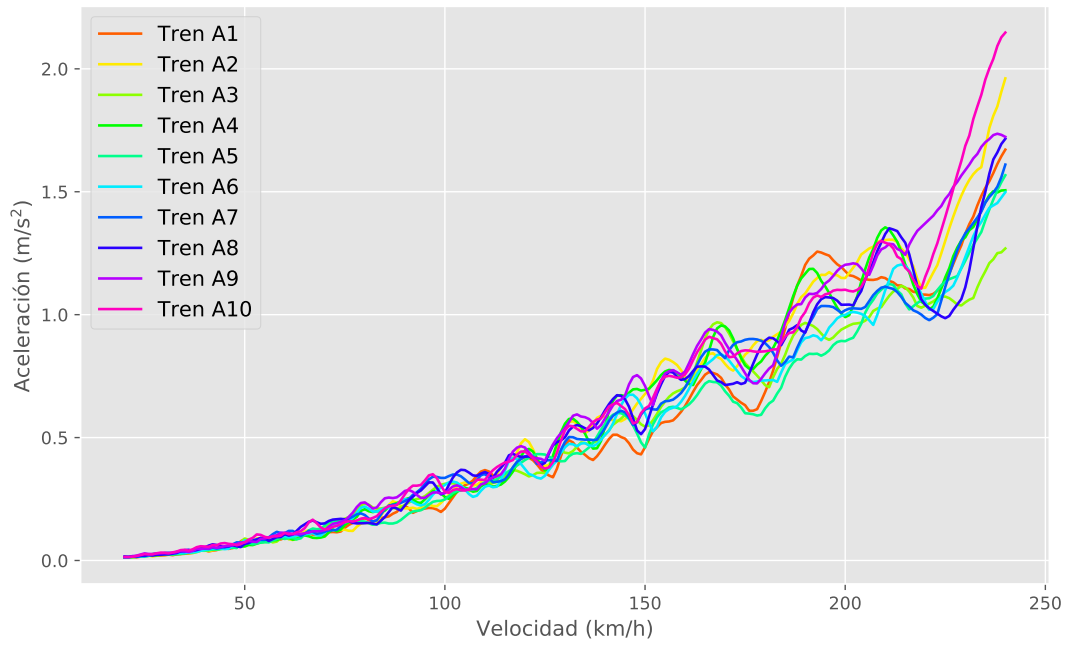


ENVOLVENTE DE ACELERACIÓN VERTICAL EN EL PUNTO 4

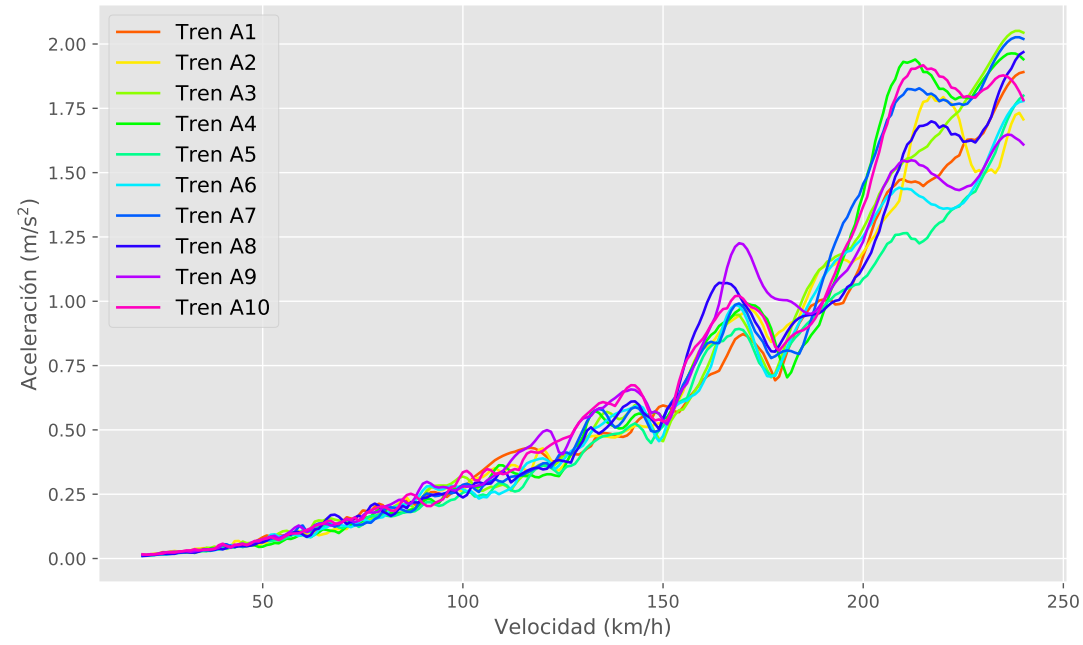

ENVOLVENTE DE ACELERACIÓN VERTICAL EN EL PUNTO 5

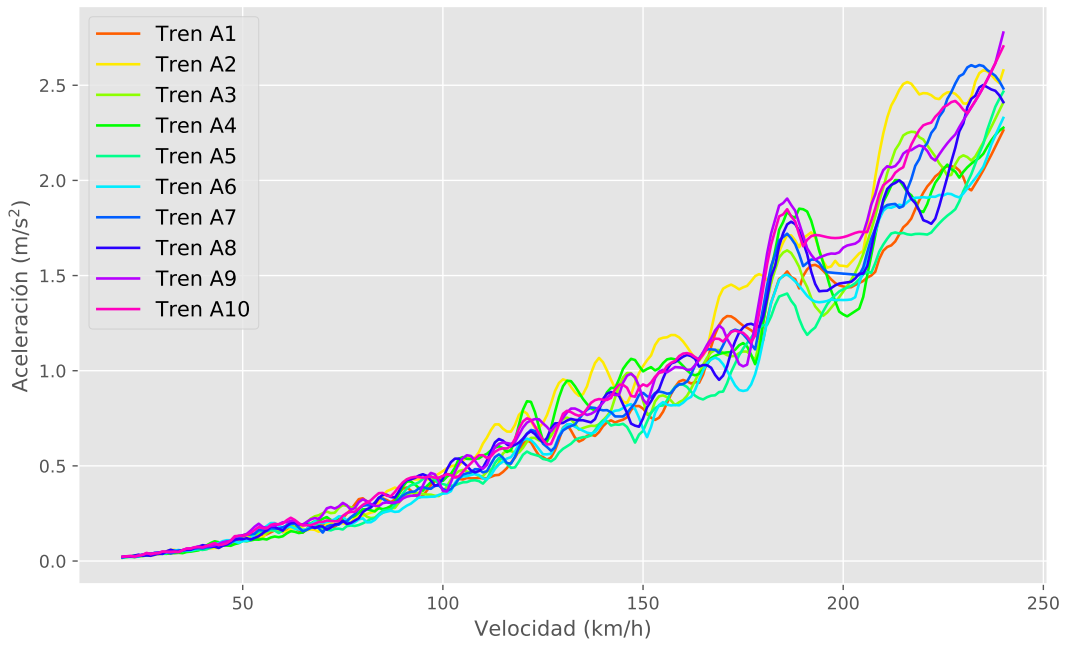


ENVOLVENTE DE ACELERACIÓN VERTICAL EN EL PUNTO 6

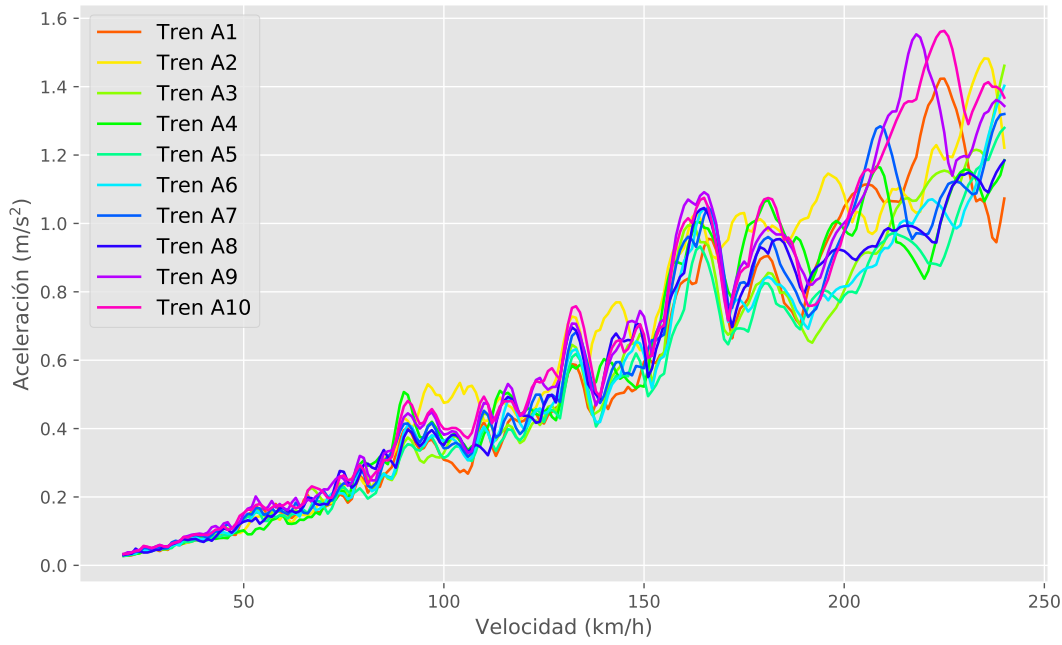

ENVOLVENTE DE ACELERACIÓN VERTICAL EN EL PUNTO 7

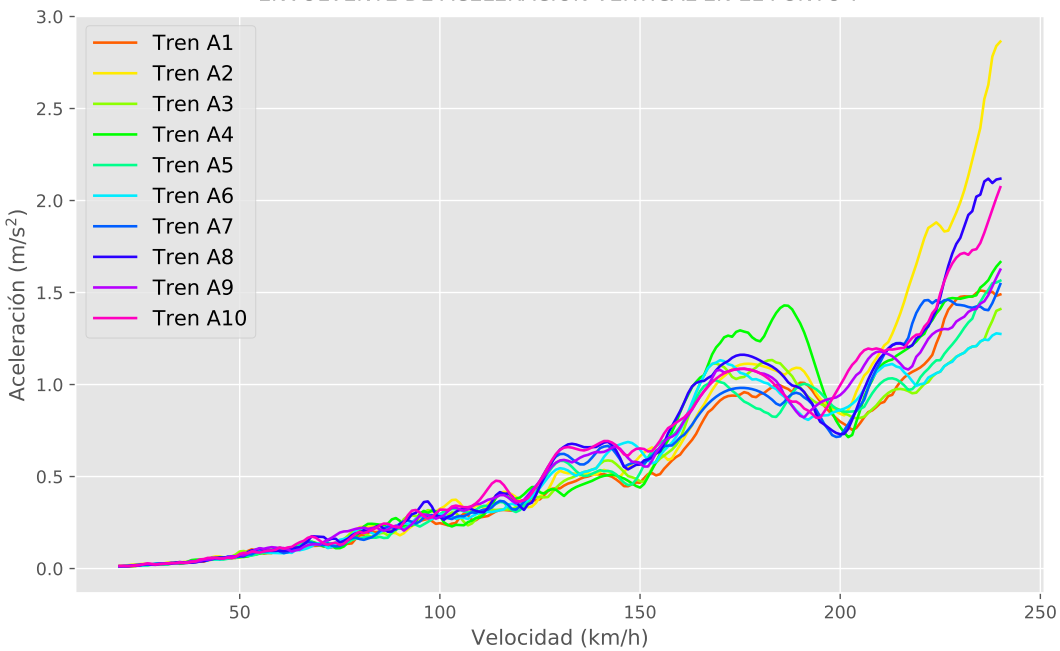


ENVOLVENTE DE ACELERACIÓN VERTICAL EN EL PUNTO 8

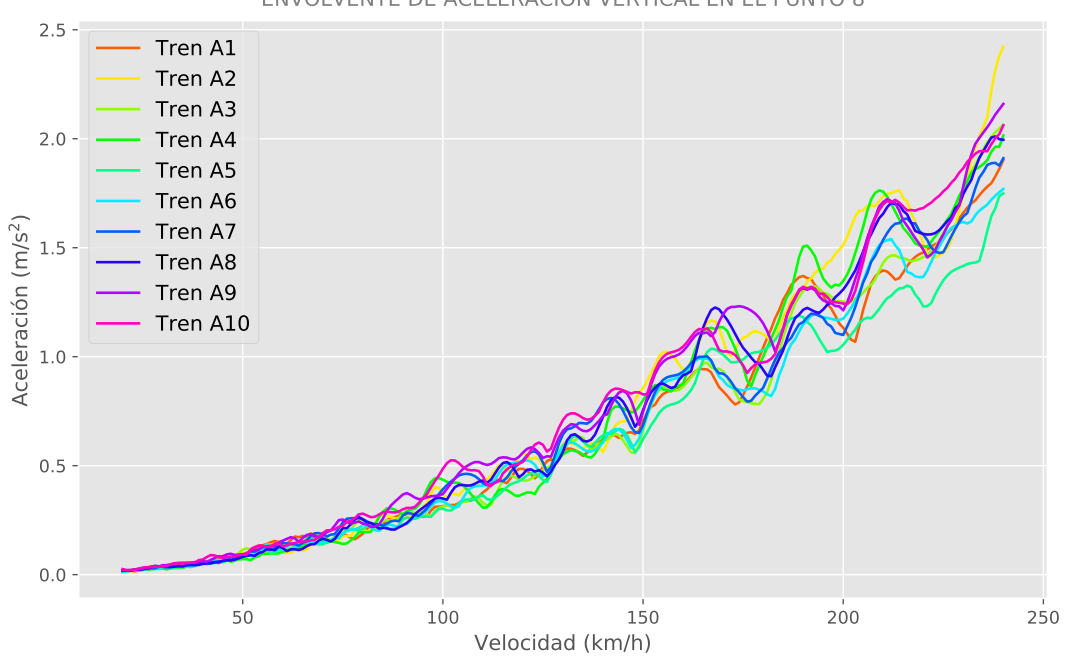

ENVOLVENTE DE ACELERACIÓN VERTICAL EN EL PUNTO 9

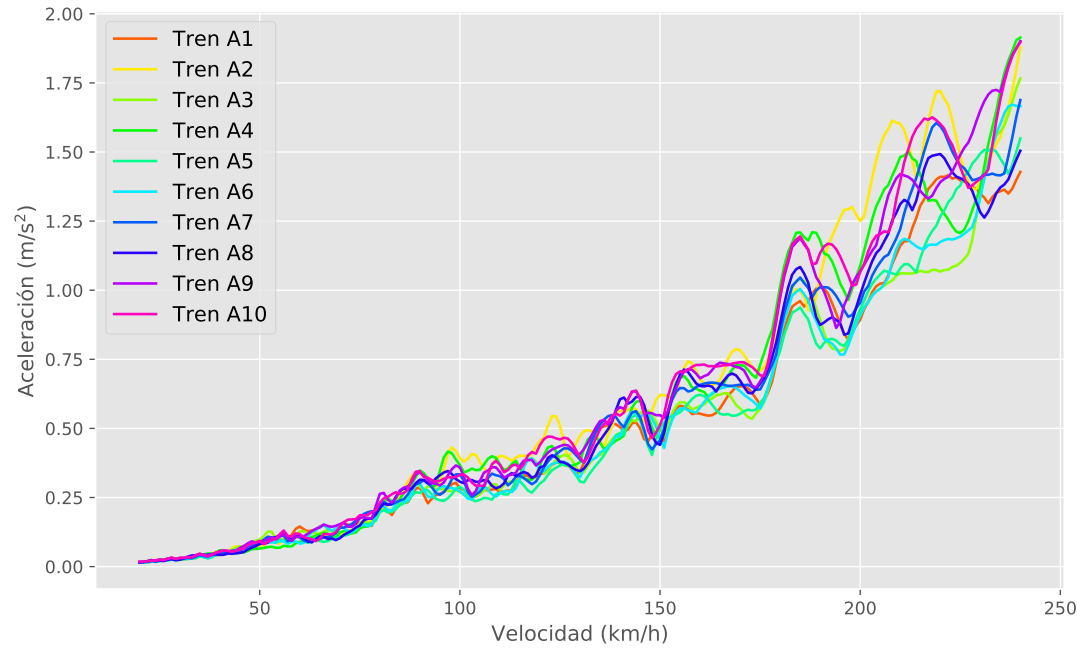




\section{A.3.2. Resultados de desplazamiento}
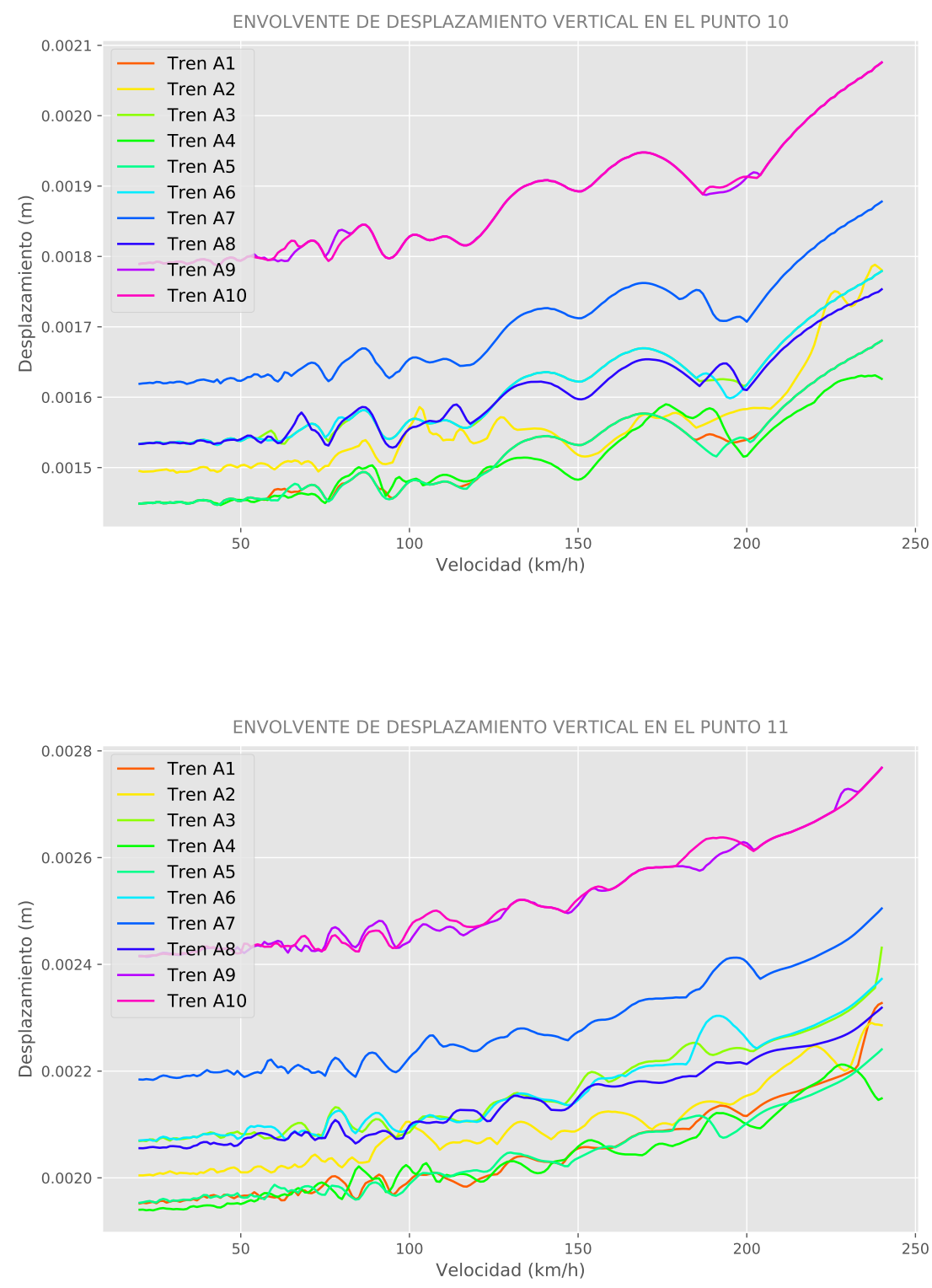


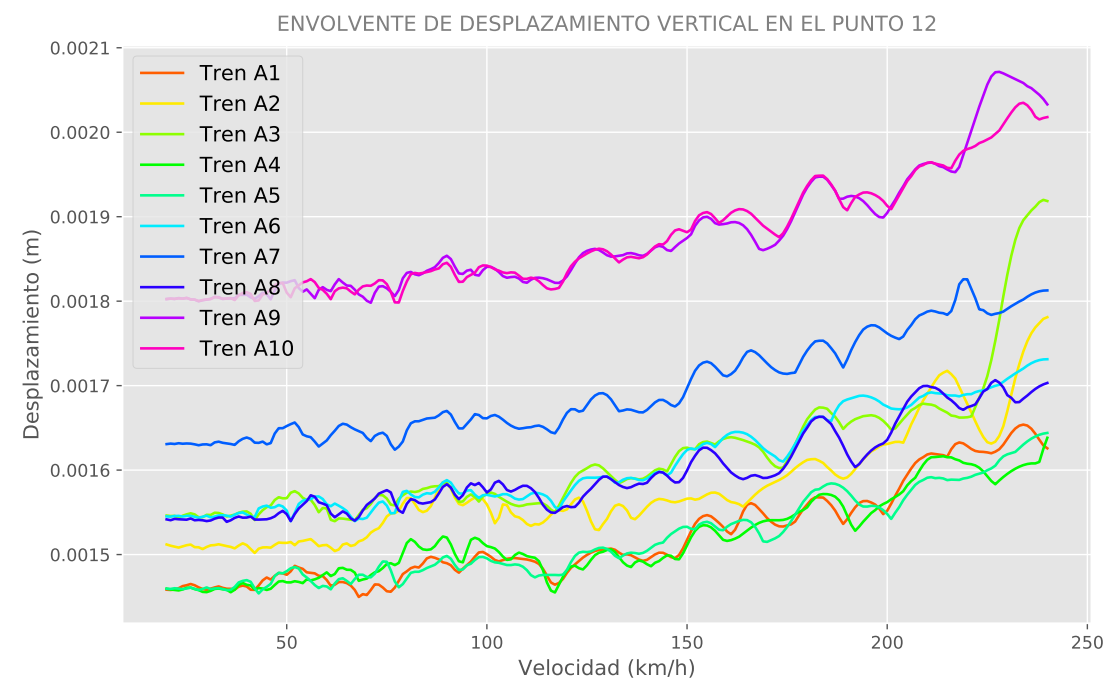

A.4. Resultados para $E_{p} / G_{s}=1000$

\section{A.4.1. Resultados de aceleración}

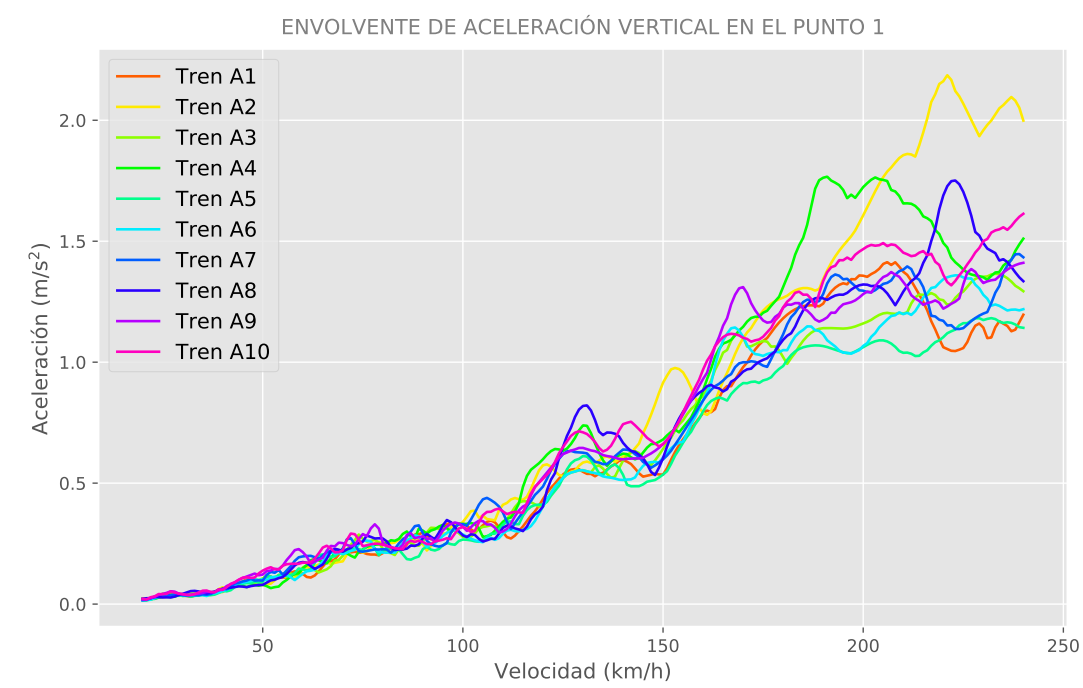


ENVOLVENTE DE ACELERACIÓN VERTICAL EN EL PUNTO 2

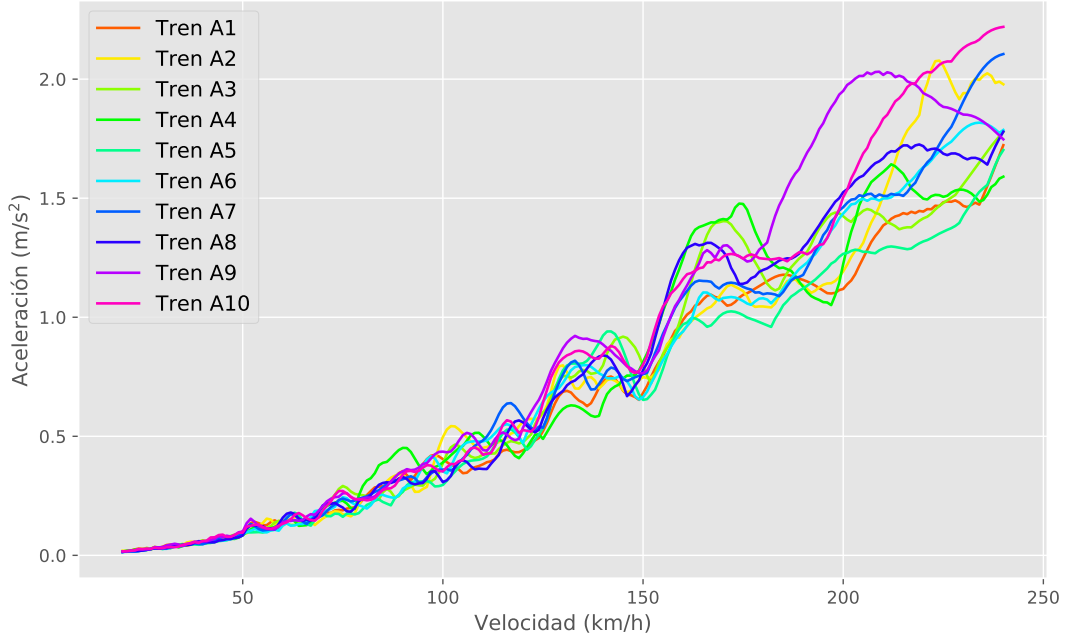

ENVOLVENTE DE ACELERACIÓN VERTICAL EN EL PUNTO 3

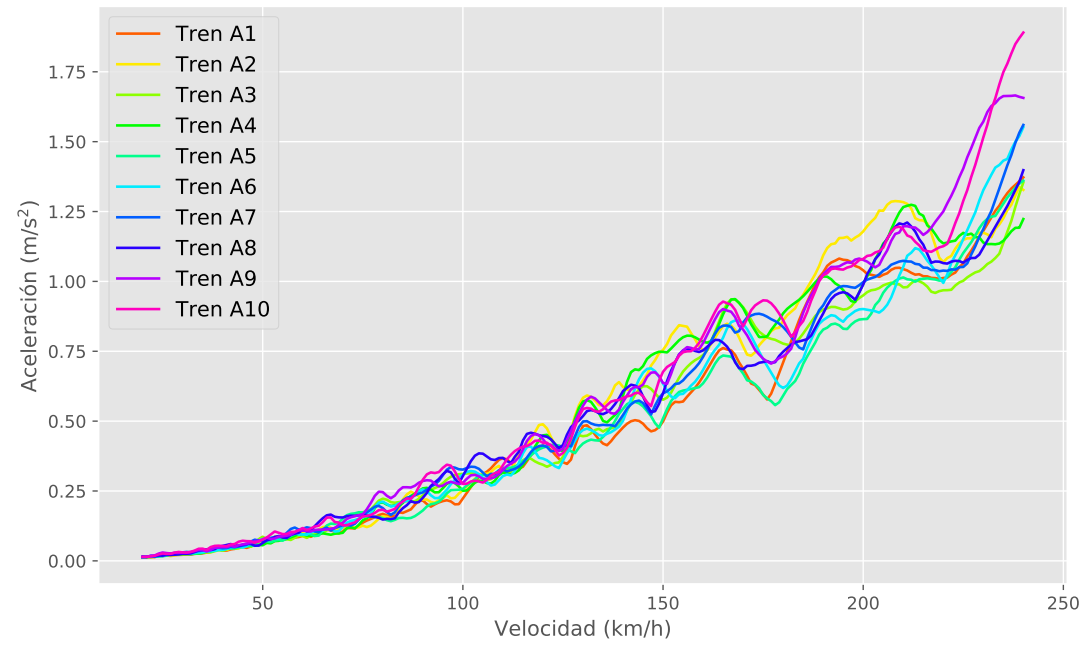


ENVOLVENTE DE ACELERACIÓN VERTICAL EN EL PUNTO 4

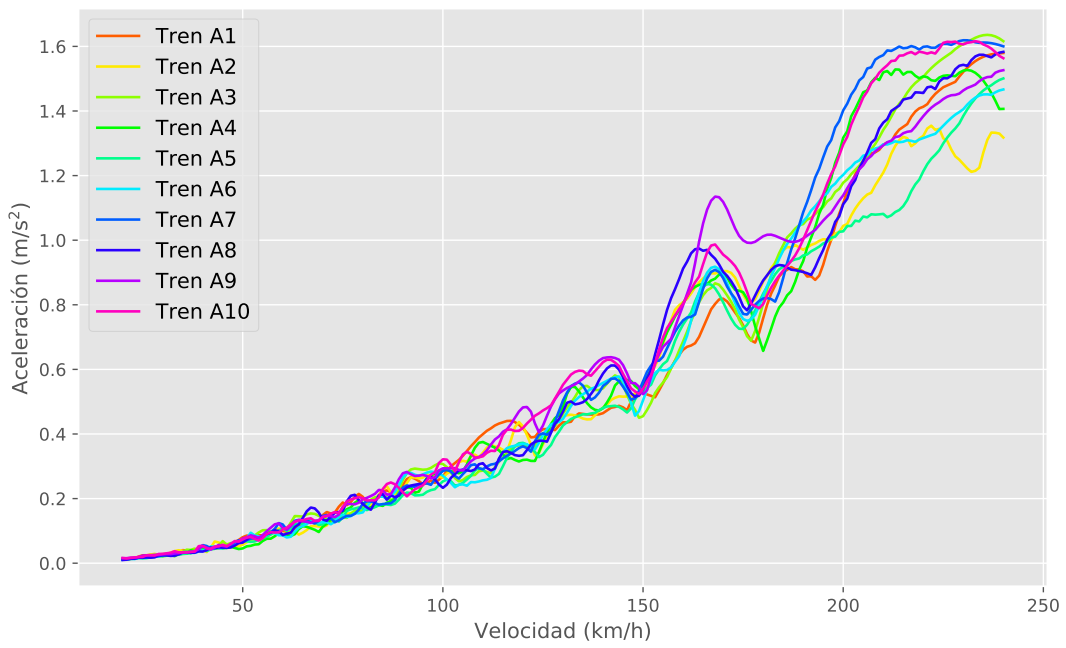

ENVOLVENTE DE ACELERACIÓN VERTICAL EN EL PUNTO 5

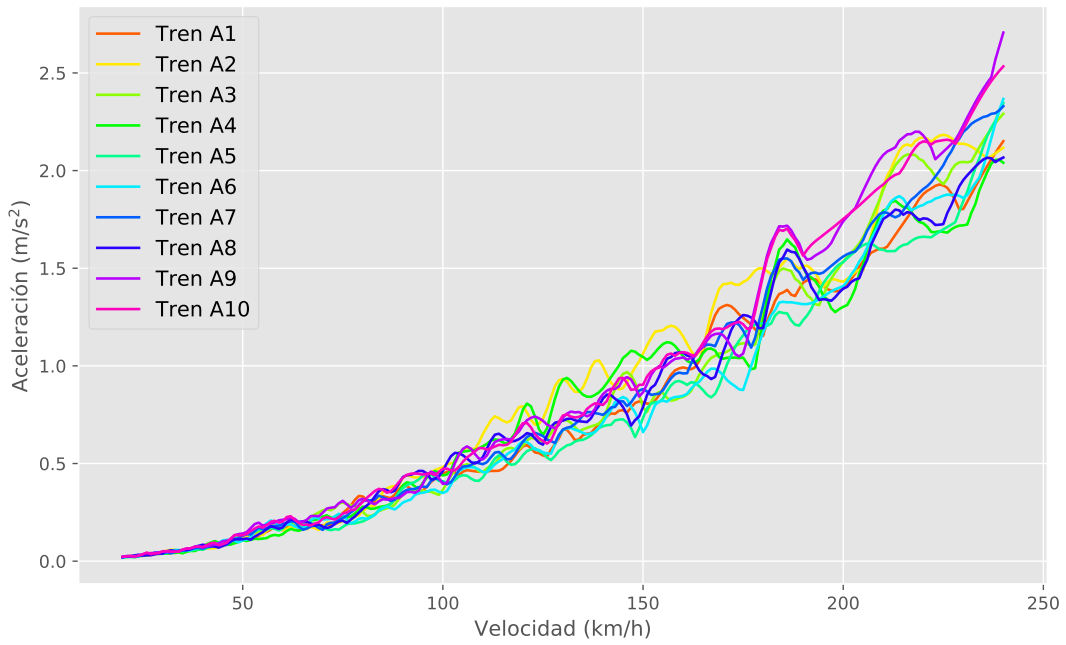


ENVOLVENTE DE ACELERACIÓN VERTICAL EN EL PUNTO 6

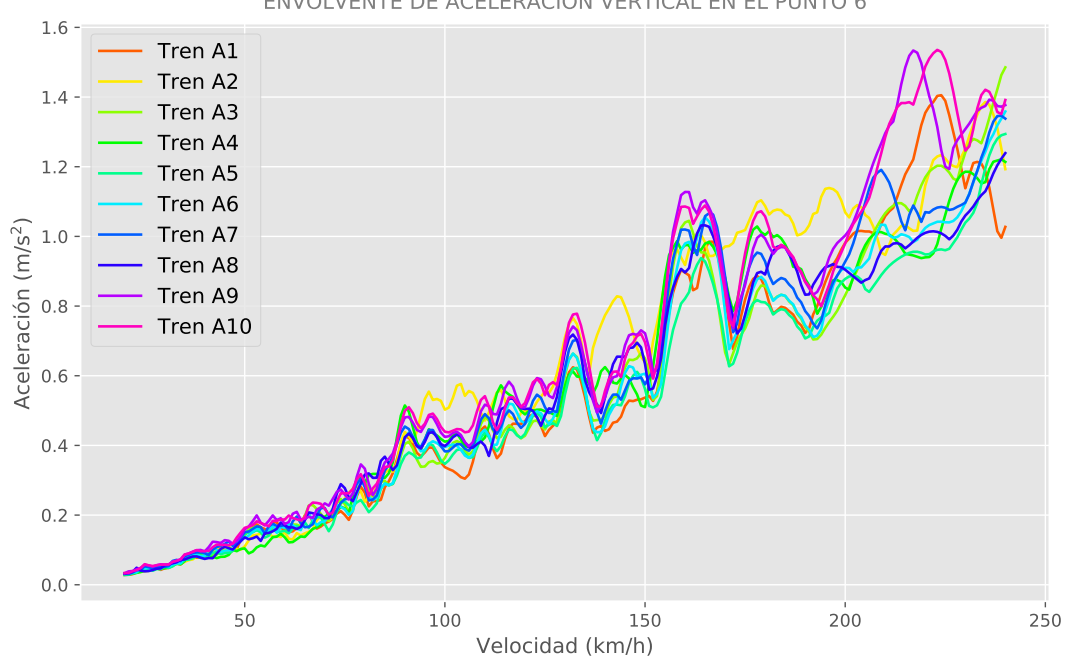

ENVOLVENTE DE ACELERACIÓN VERTICAL EN EL PUNTO 7

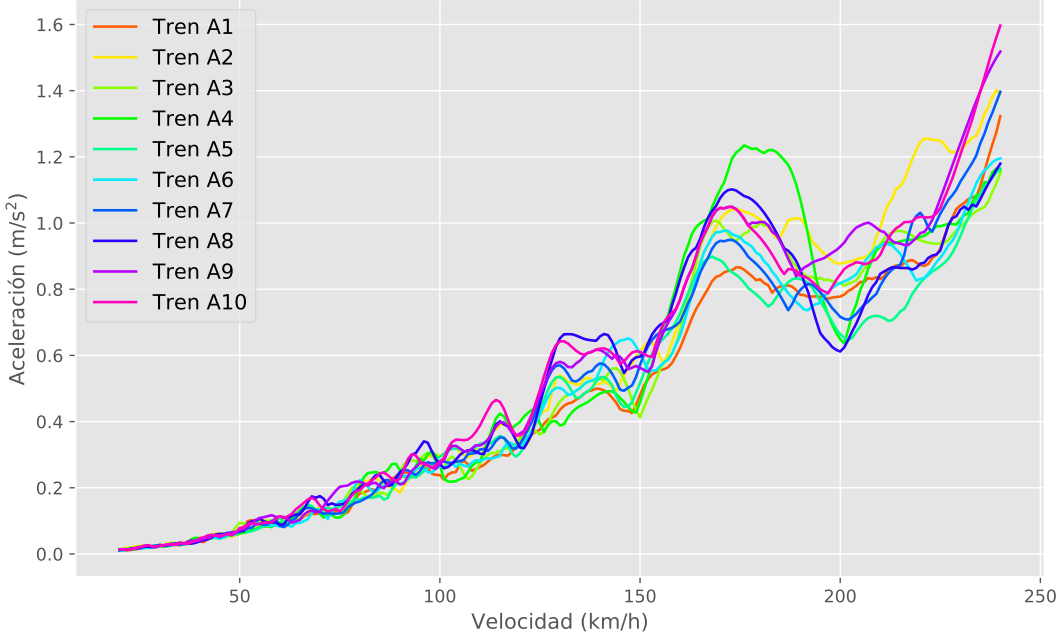


ENVOLVENTE DE ACELERACIÓN VERTICAL EN EL PUNTO 8

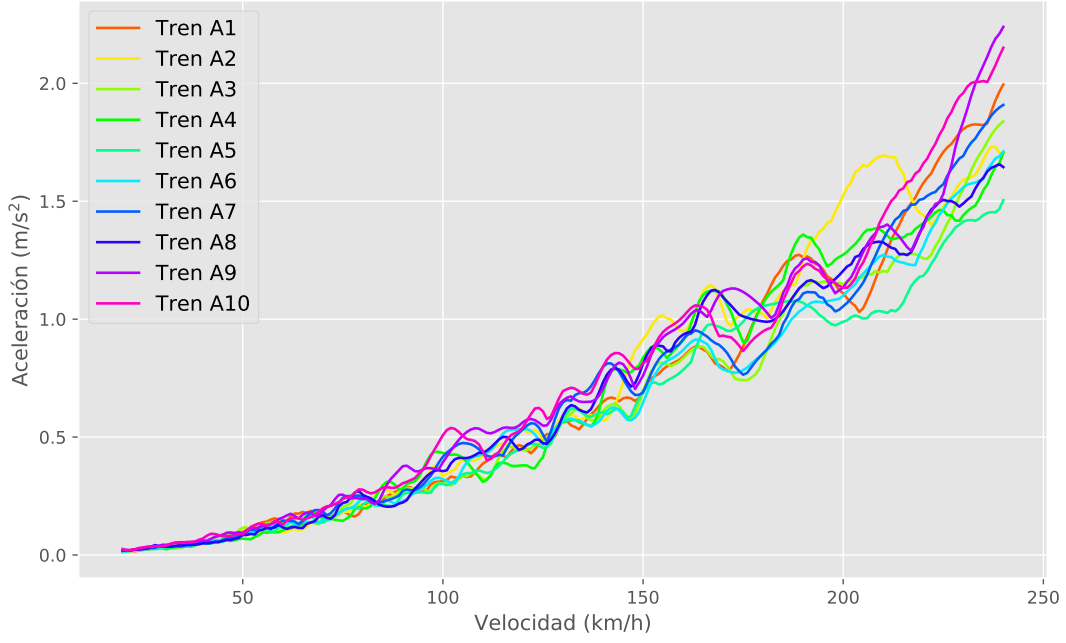

ENVOLVENTE DE ACELERACIÓN VERTICAL EN EL PUNTO 9

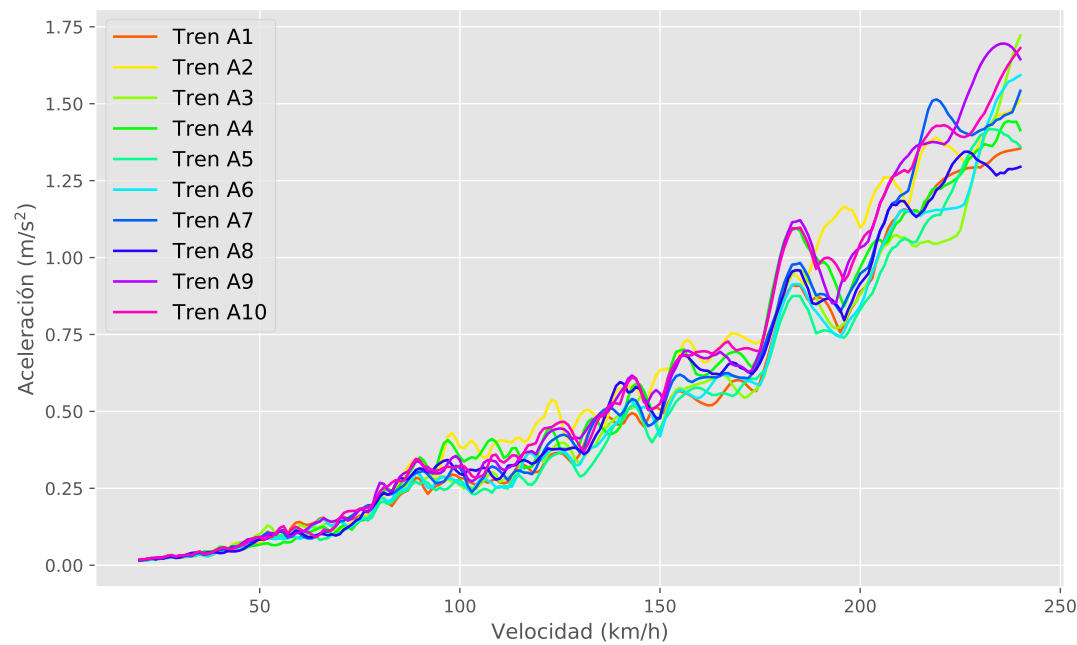




\section{A.4.2. Resultados de desplazamiento}

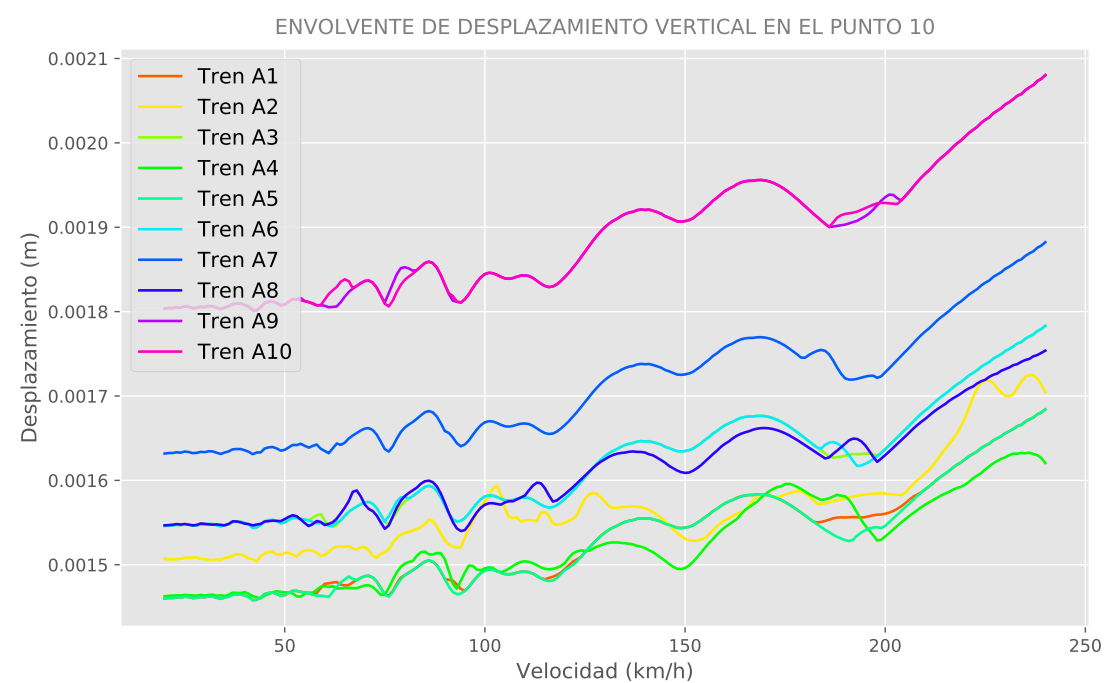

ENVOLVENTE DE DESPLAZAMIENTO VERTICAL EN EL PUNTO 11

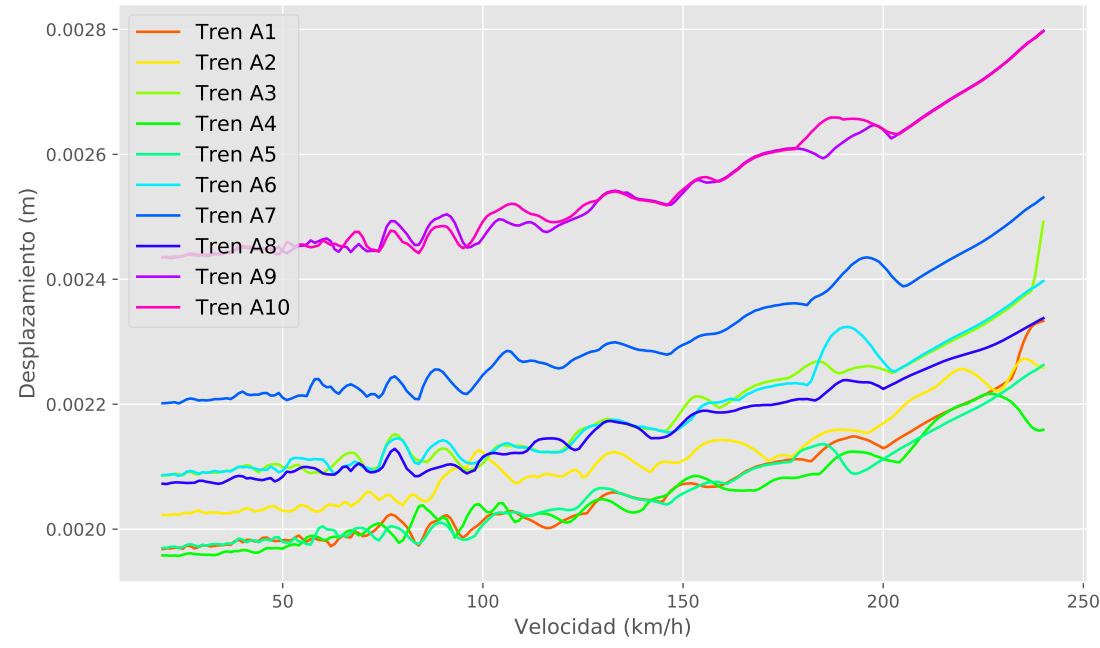


ENVOLVENTE DE DESPLAZAMIENTO VERTICAL EN EL PUNTO 12

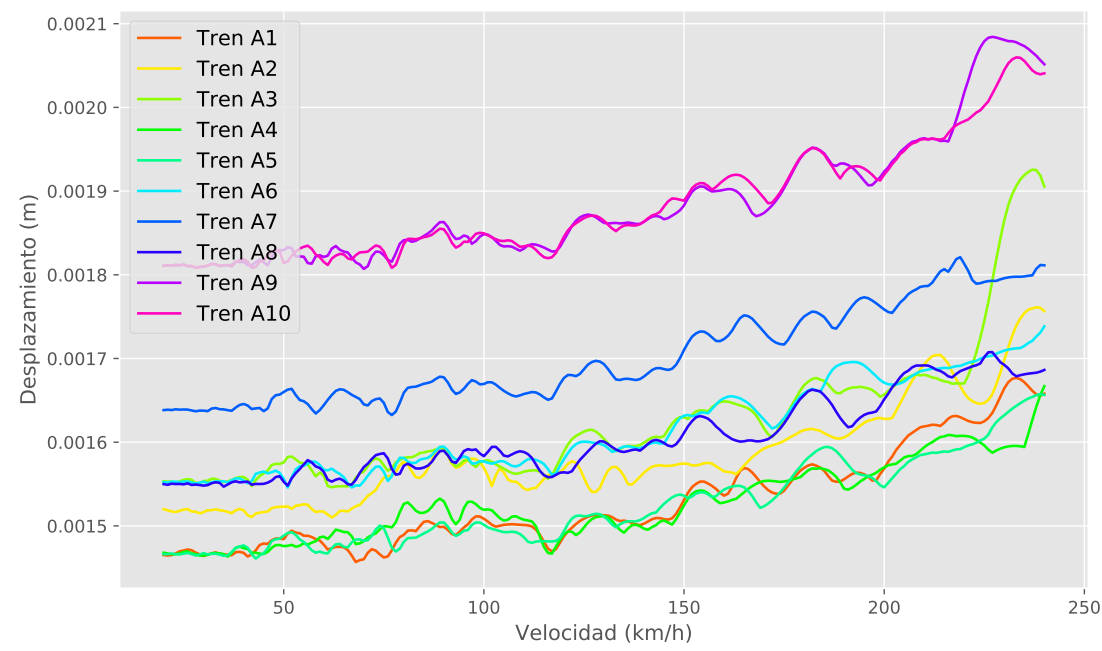

A.5. Resultados para $E_{p} / G_{S}=2500$

\section{A.5.1. Resultados de aceleración}

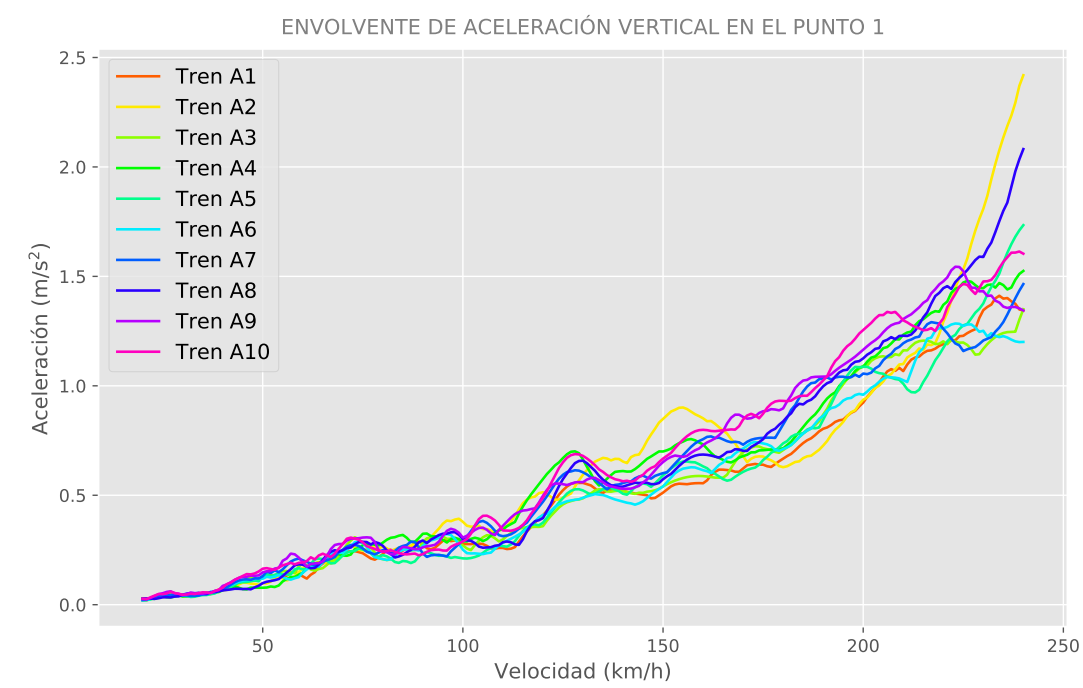


ENVOLVENTE DE ACELERACIÓN VERTICAL EN EL PUNTO 2

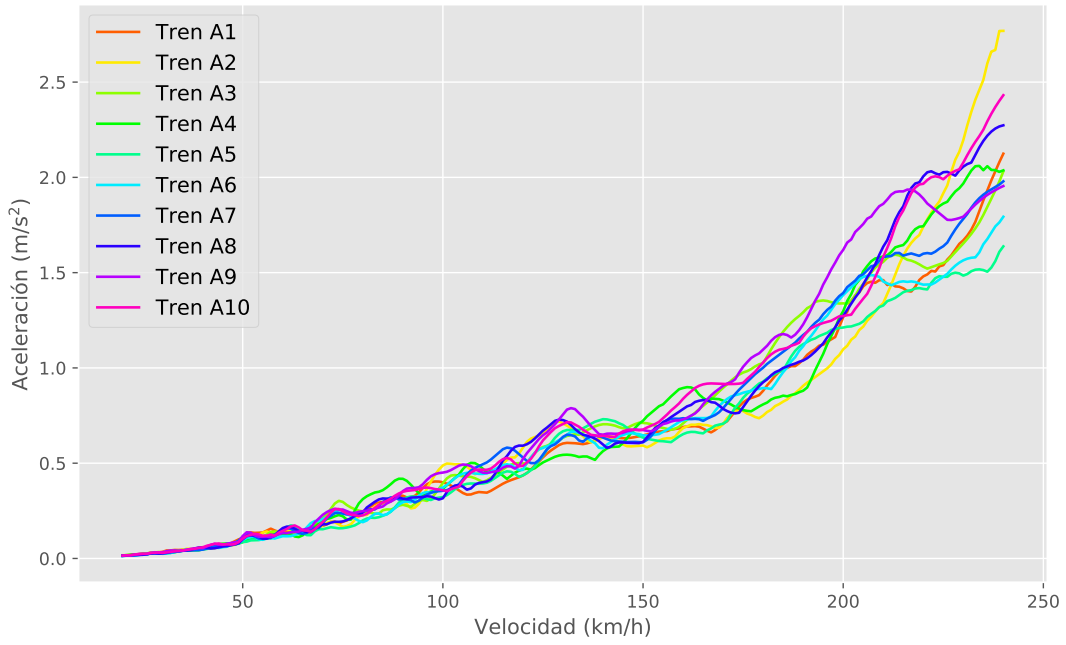

ENVOLVENTE DE ACELERACIÓN VERTICAL EN EL PUNTO 3

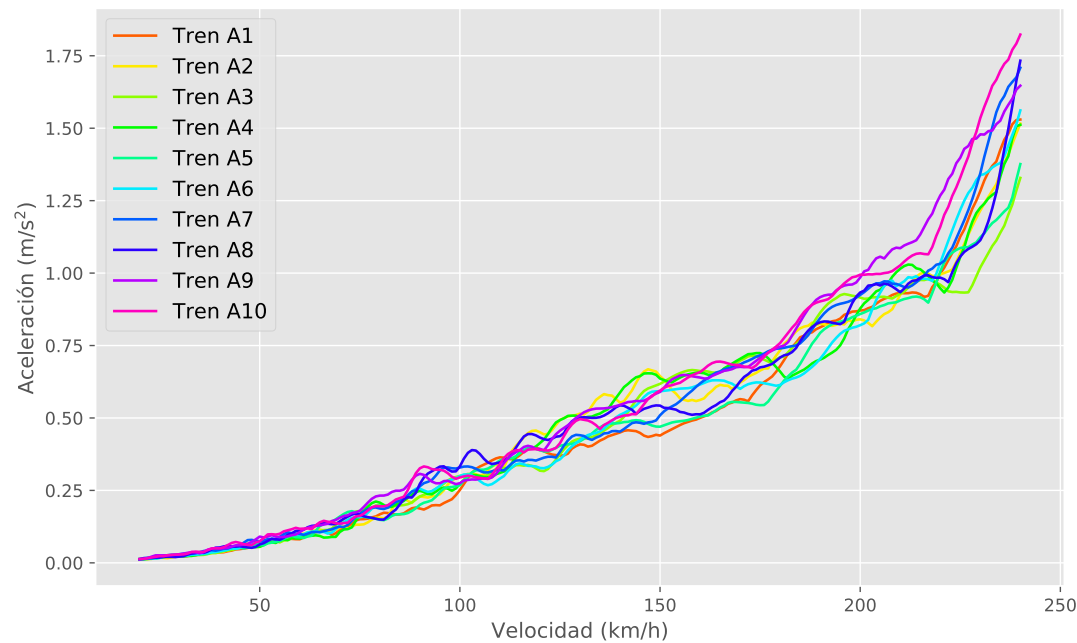


ENVOLVENTE DE ACELERACIÓN VERTICAL EN EL PUNTO 4

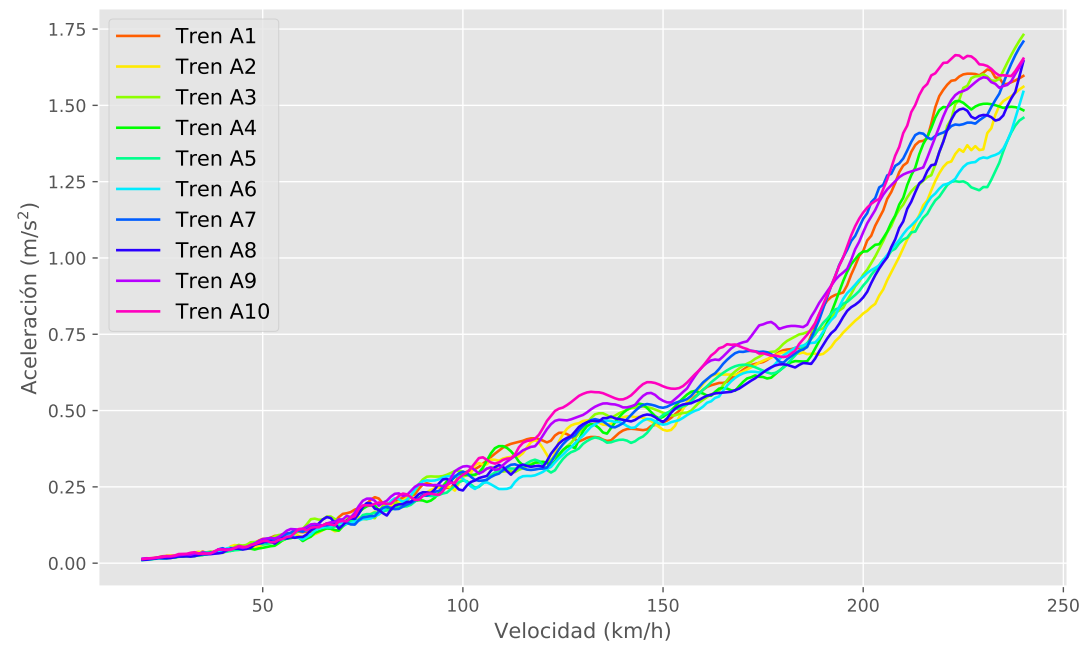

ENVOLVENTE DE ACELERACIÓN VERTICAL EN EL PUNTO 5

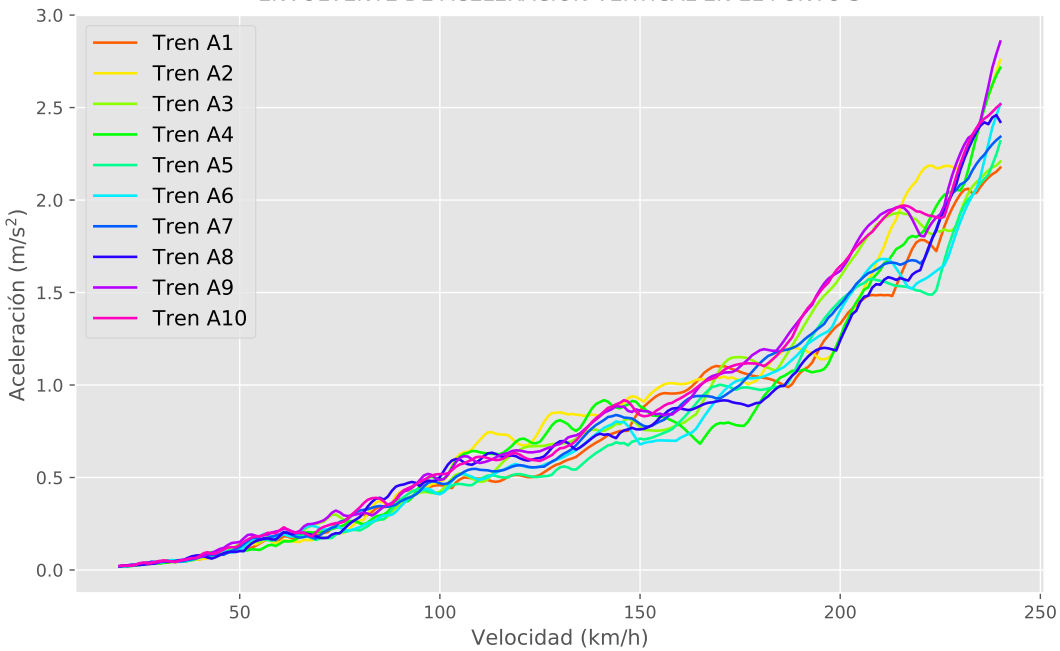


ENVOLVENTE DE ACELERACIÓN VERTICAL EN EL PUNTO 6

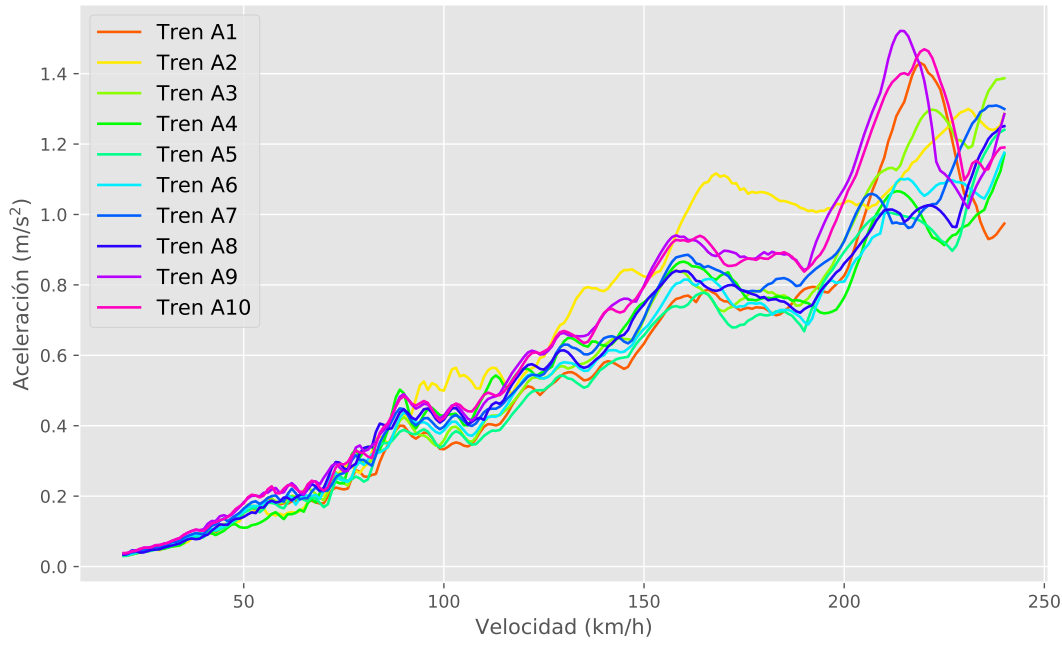

ENVOLVENTE DE ACELERACIÓN VERTICAL EN EL PUNTO 7

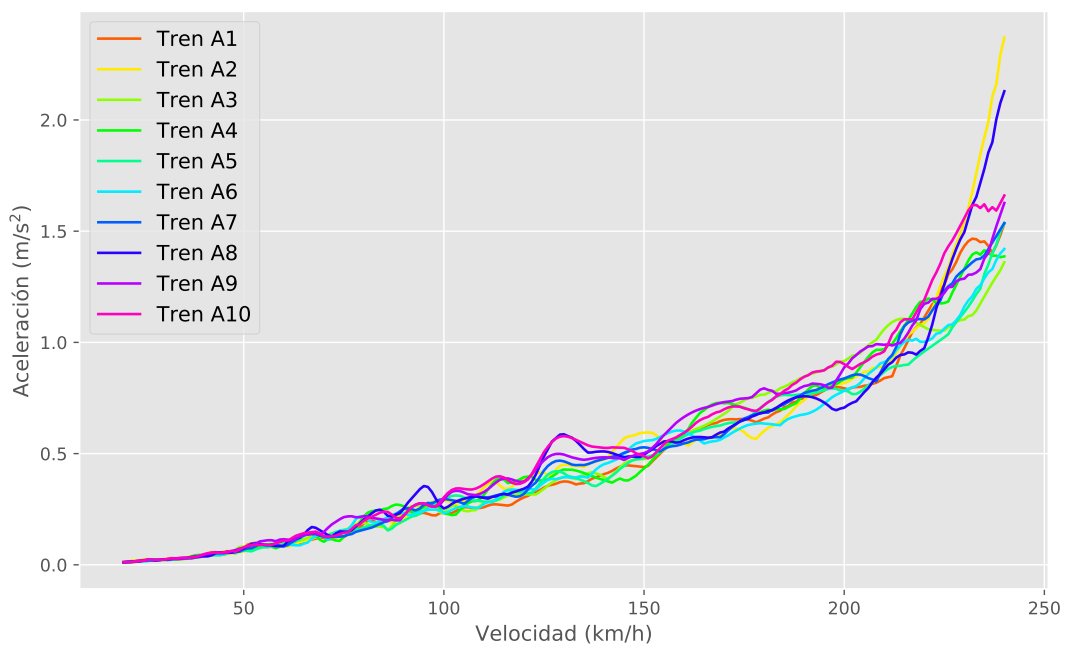


ENVOLVENTE DE ACELERACIÓN VERTICAL EN EL PUNTO 8

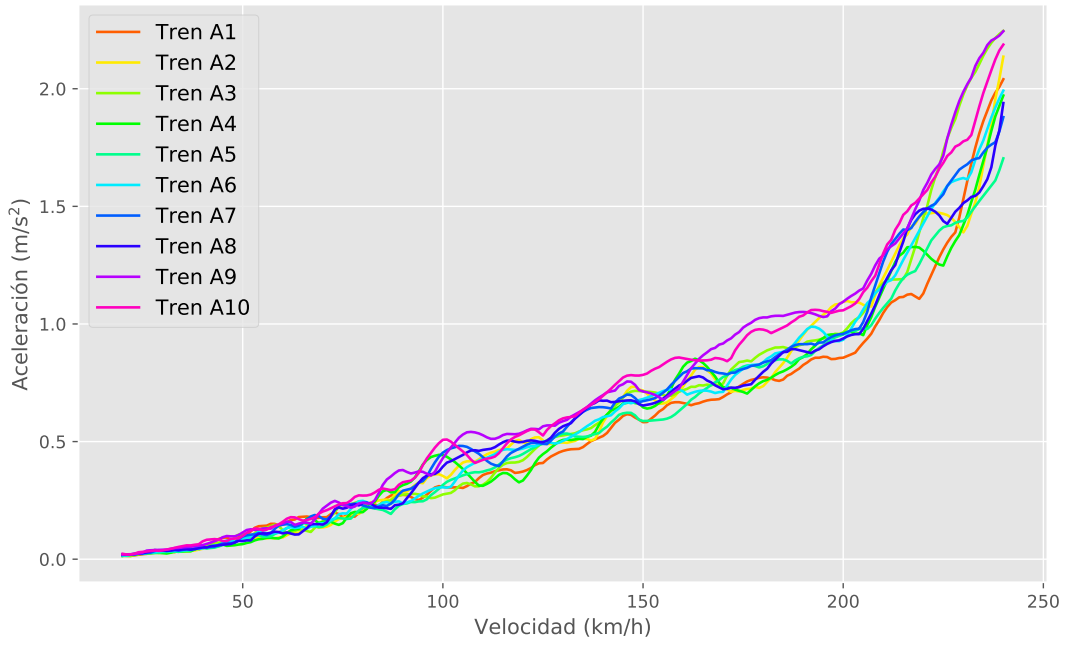

ENVOLVENTE DE ACELERACIÓN VERTICAL EN EL PUNTO 9

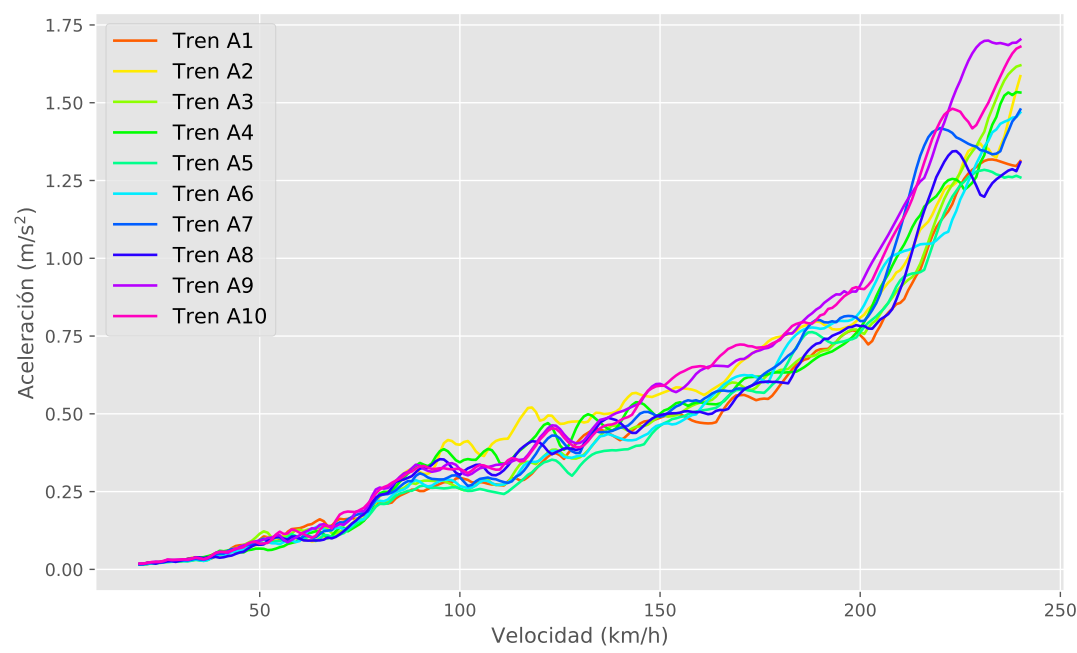




\section{A.5.2. Resultados de desplazamiento}
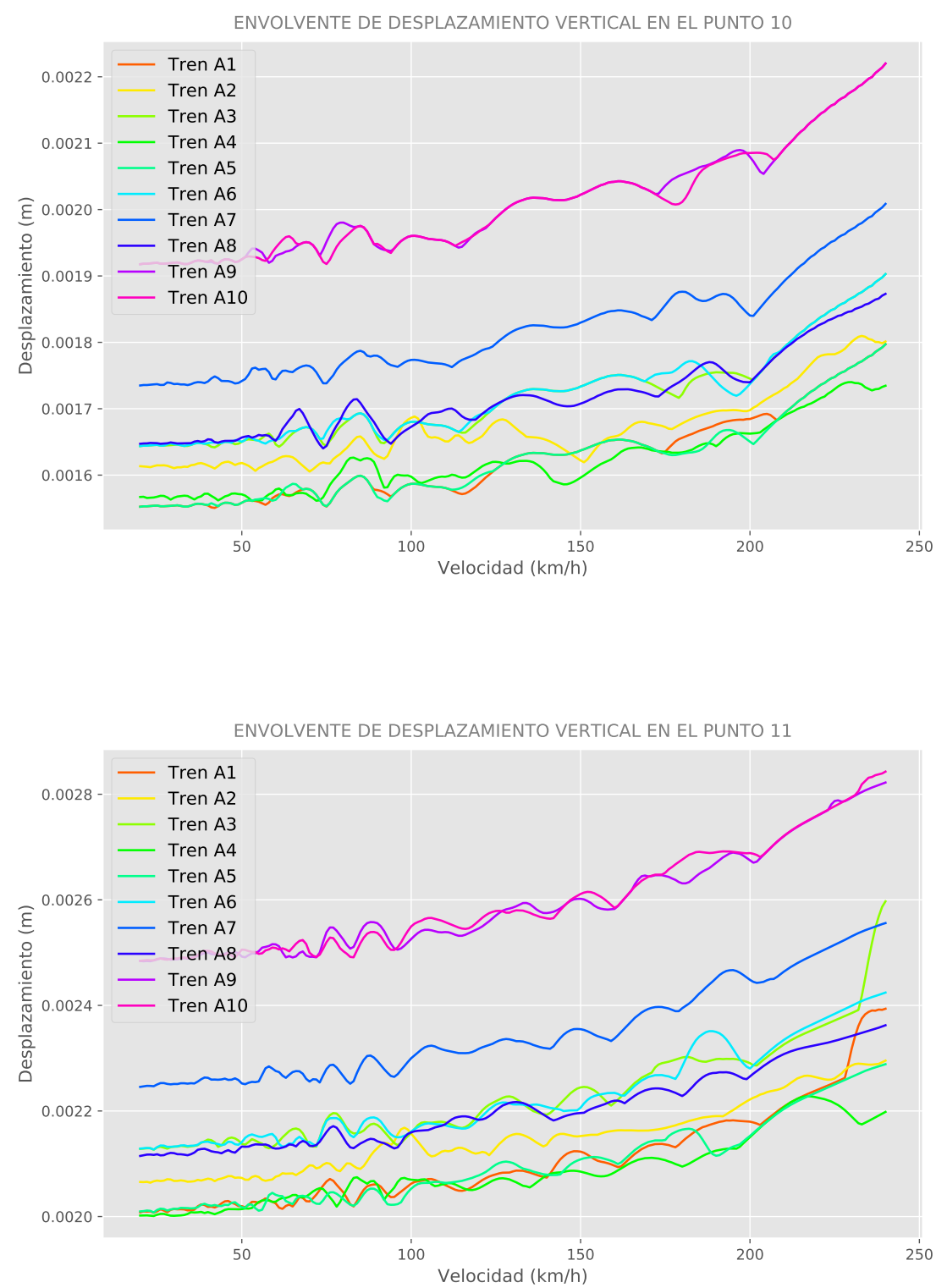
ENVOLVENTE DE DESPLAZAMIENTO VERTICAL EN EL PUNTO 12

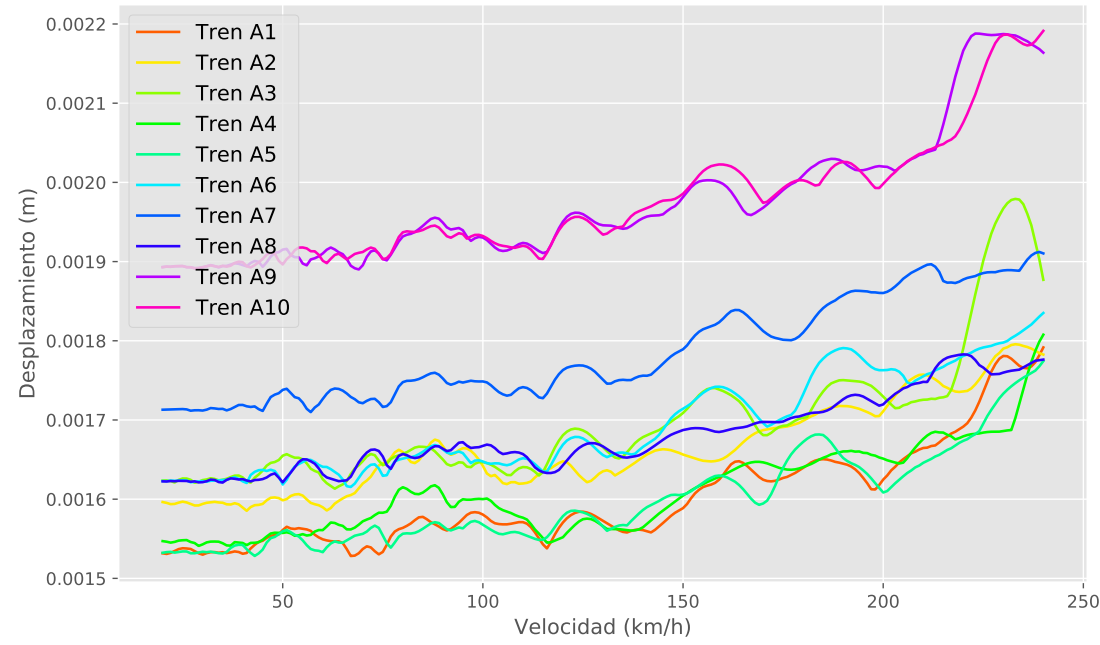

A.6. Resultados para $E_{p} / G_{s}=5000$

\section{A.6.1. Resultados de aceleración}

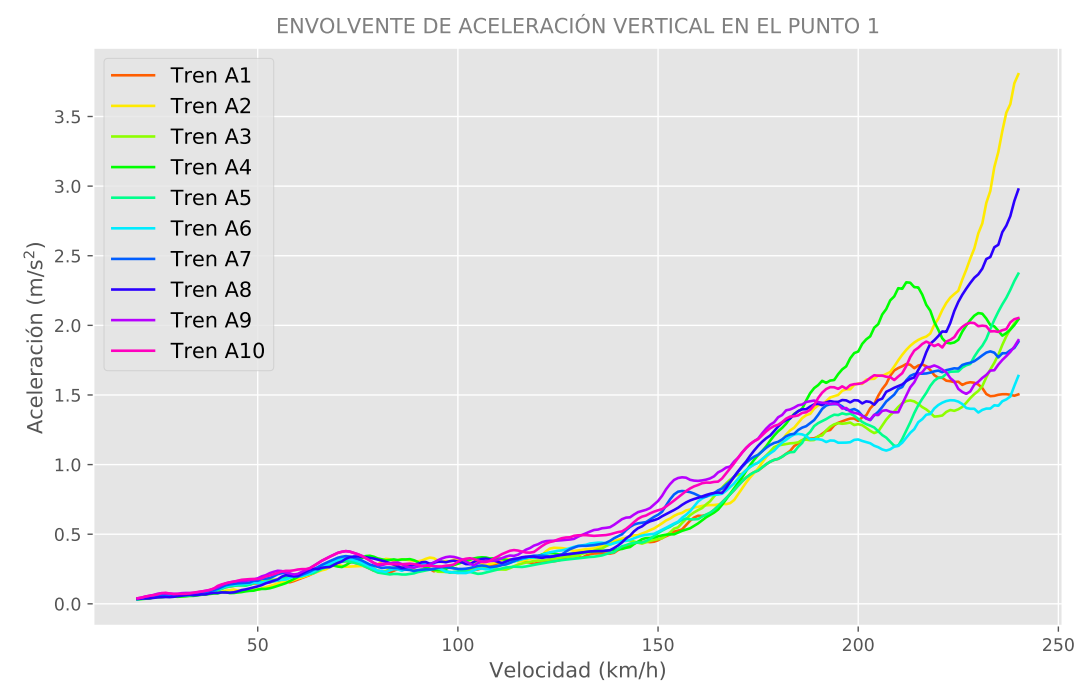


ENVOLVENTE DE ACELERACIÓN VERTICAL EN EL PUNTO 2

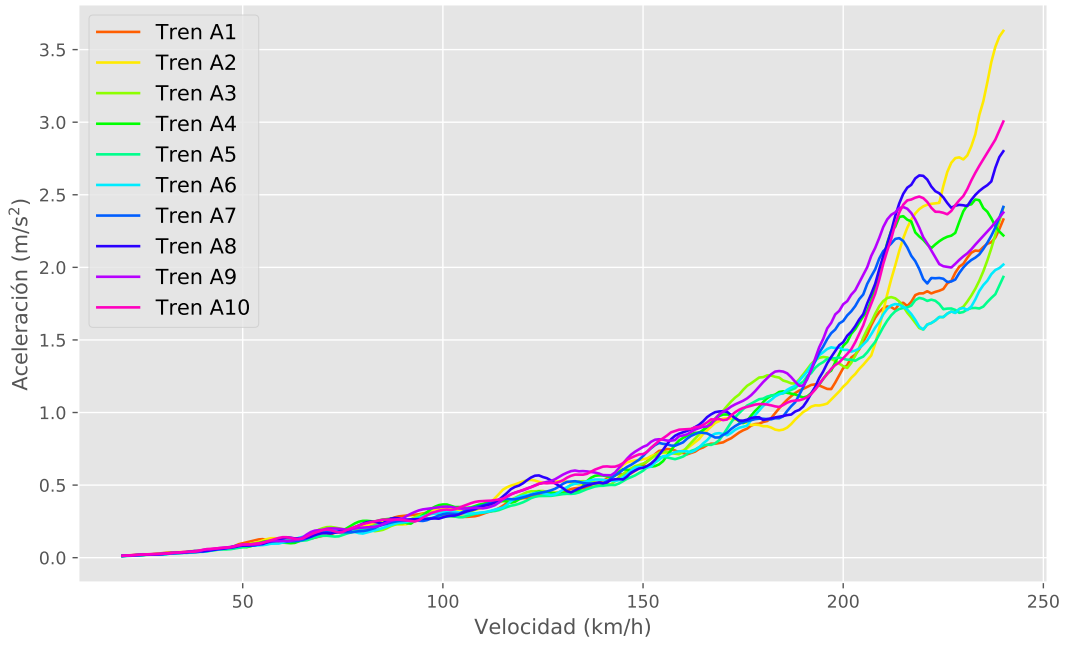

ENVOLVENTE DE ACELERACIÓN VERTICAL EN EL PUNTO 3

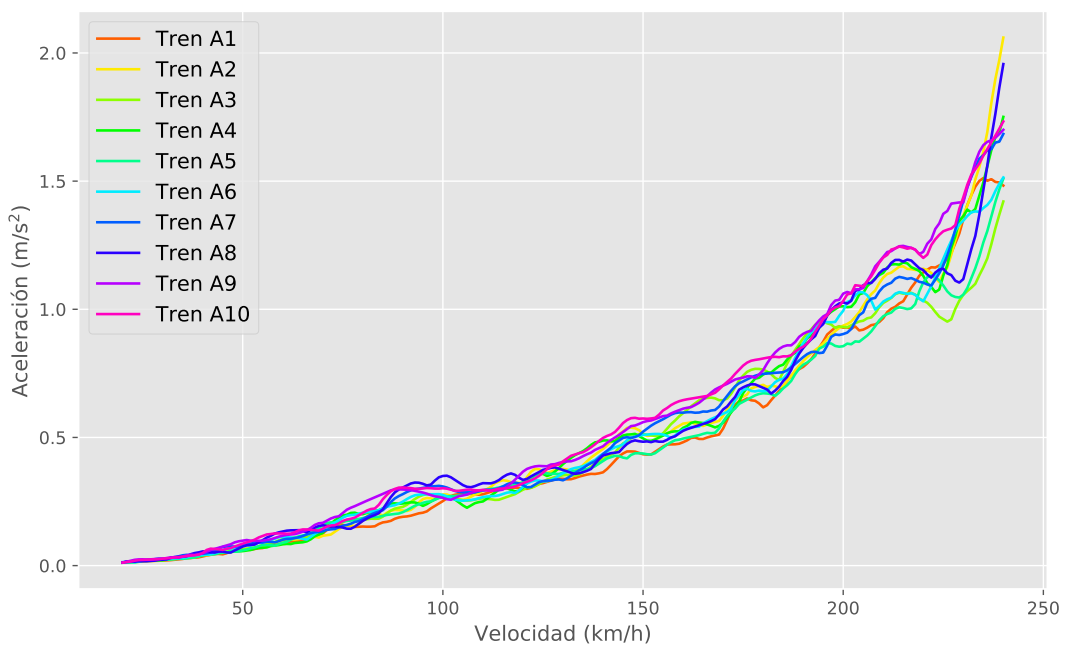


ENVOLVENTE DE ACELERACIÓN VERTICAL EN EL PUNTO 4

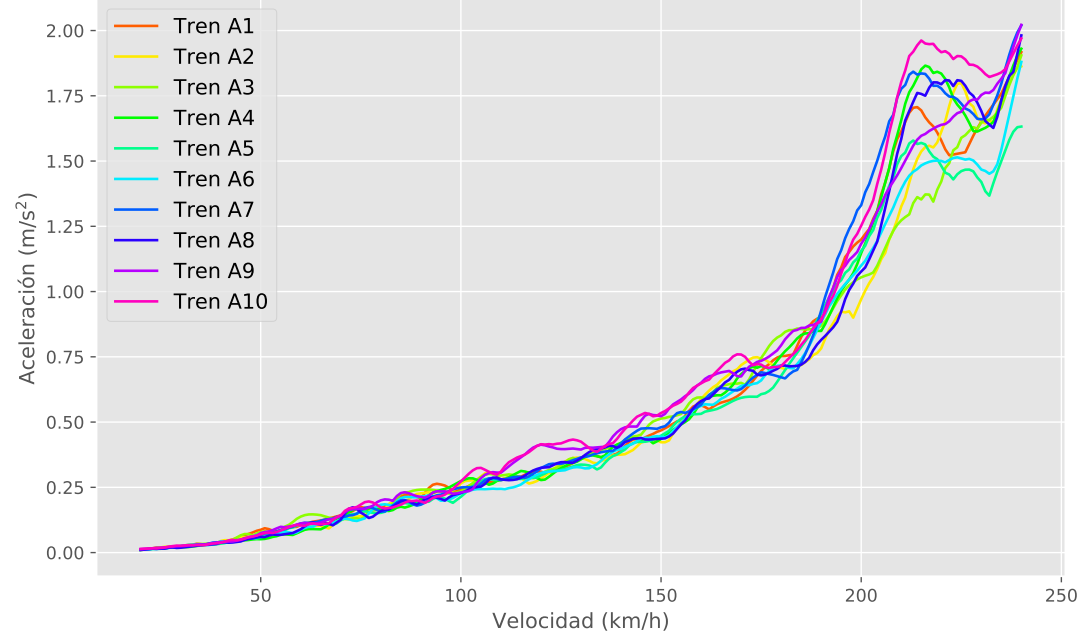

ENVOLVENTE DE ACELERACIÓN VERTICAL EN EL PUNTO 5

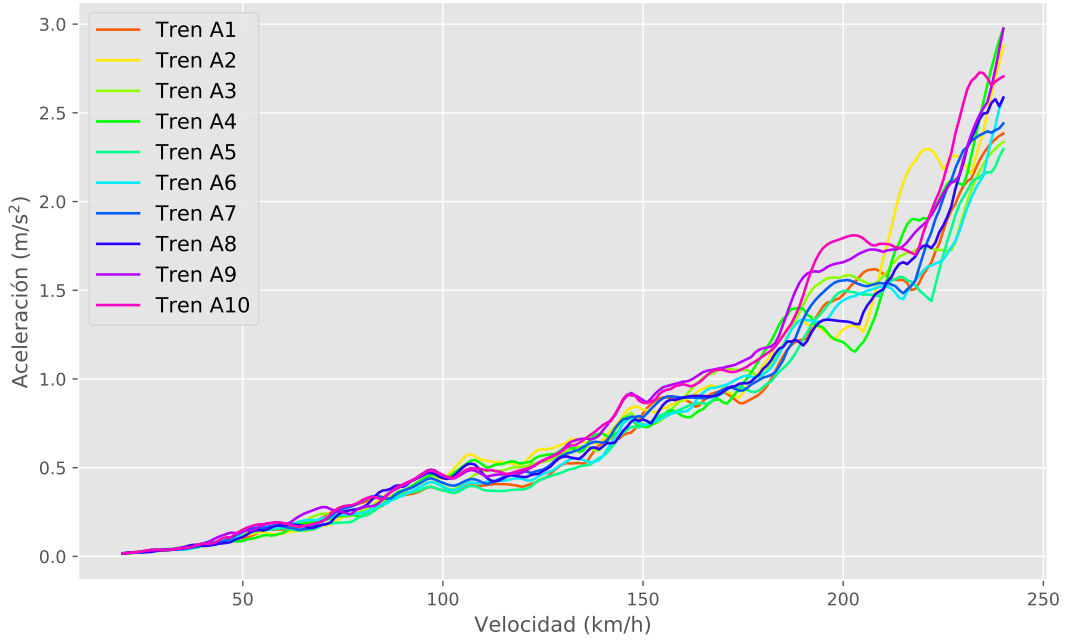


ENVOLVENTE DE ACELERACIÓN VERTICAL EN EL PUNTO 6

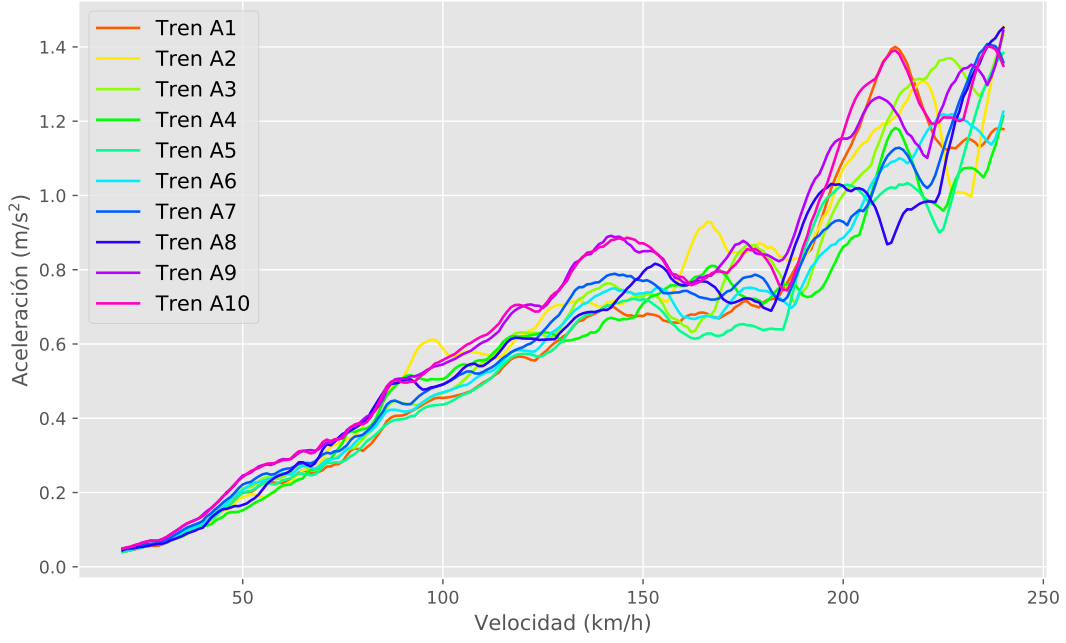

ENVOLVENTE DE ACELERACIÓN VERTICAL EN EL PUNTO 7

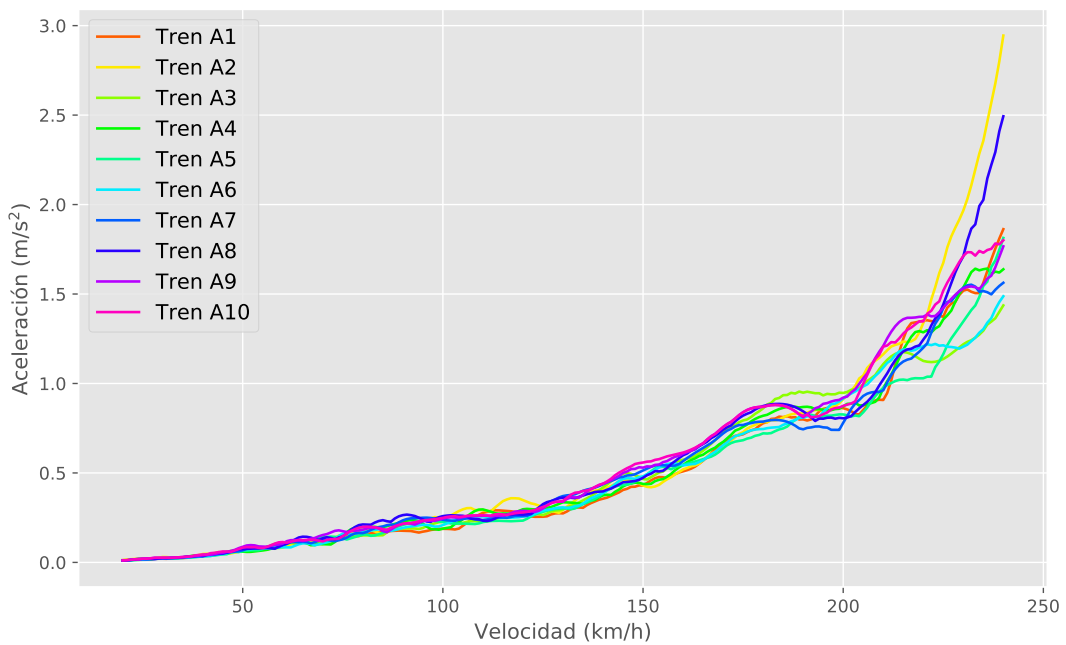


ENVOLVENTE DE ACELERACIÓN VERTICAL EN EL PUNTO 8

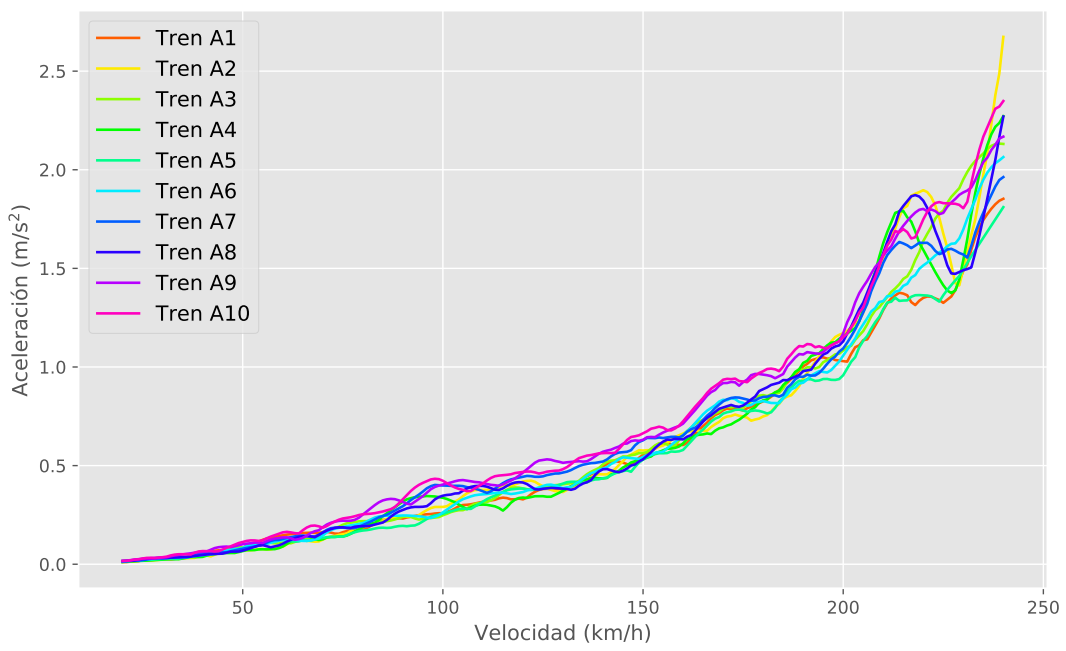

ENVOLVENTE DE ACELERACIÓN VERTICAL EN EL PUNTO 9

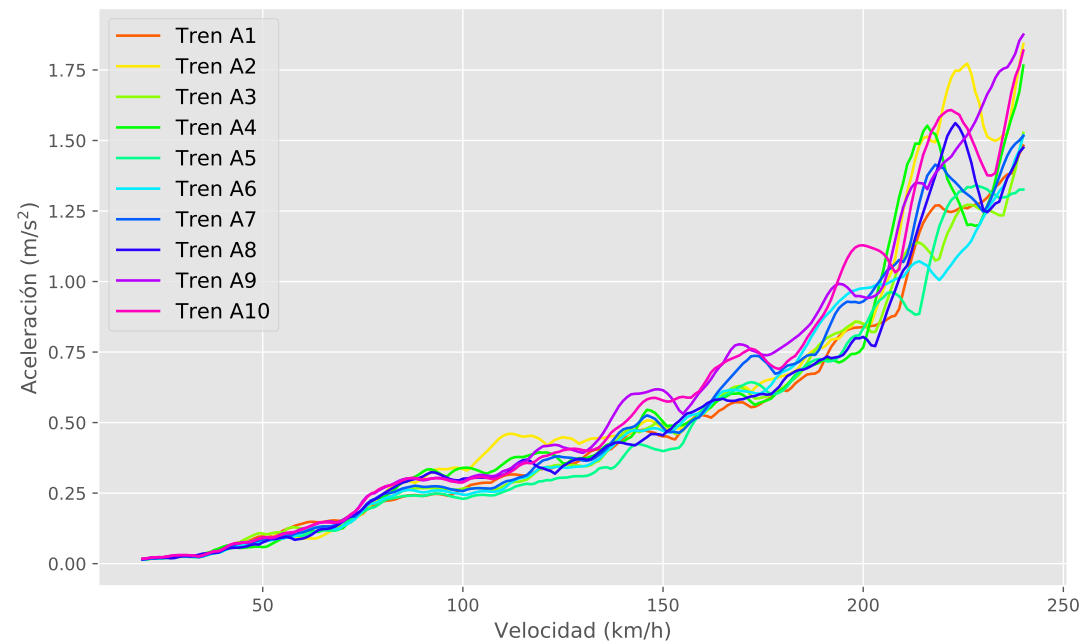




\section{A.6.2. Resultados de desplazamiento}
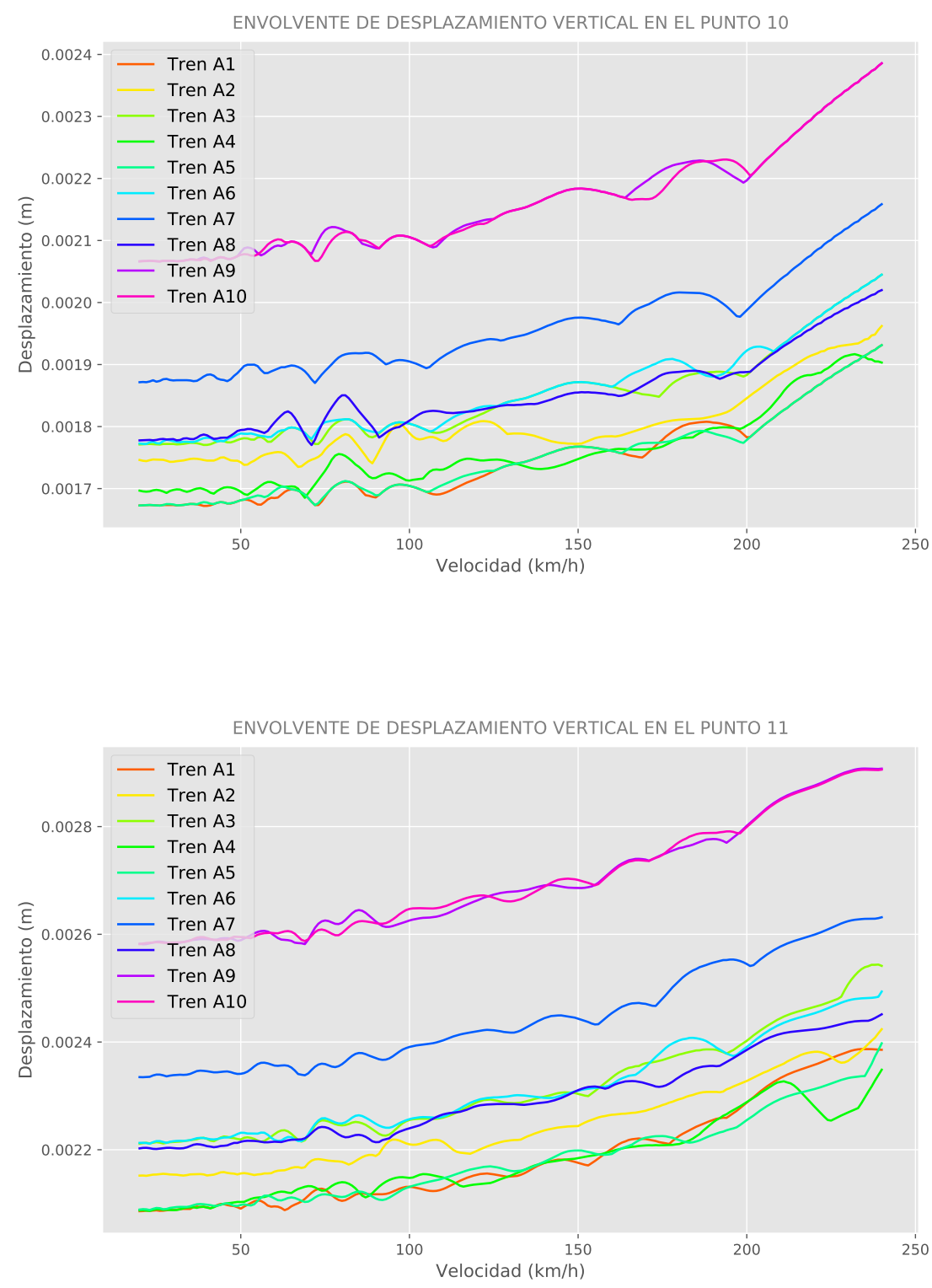
ENVOLVENTE DE DESPLAZAMIENTO VERTICAL EN EL PUNTO 12

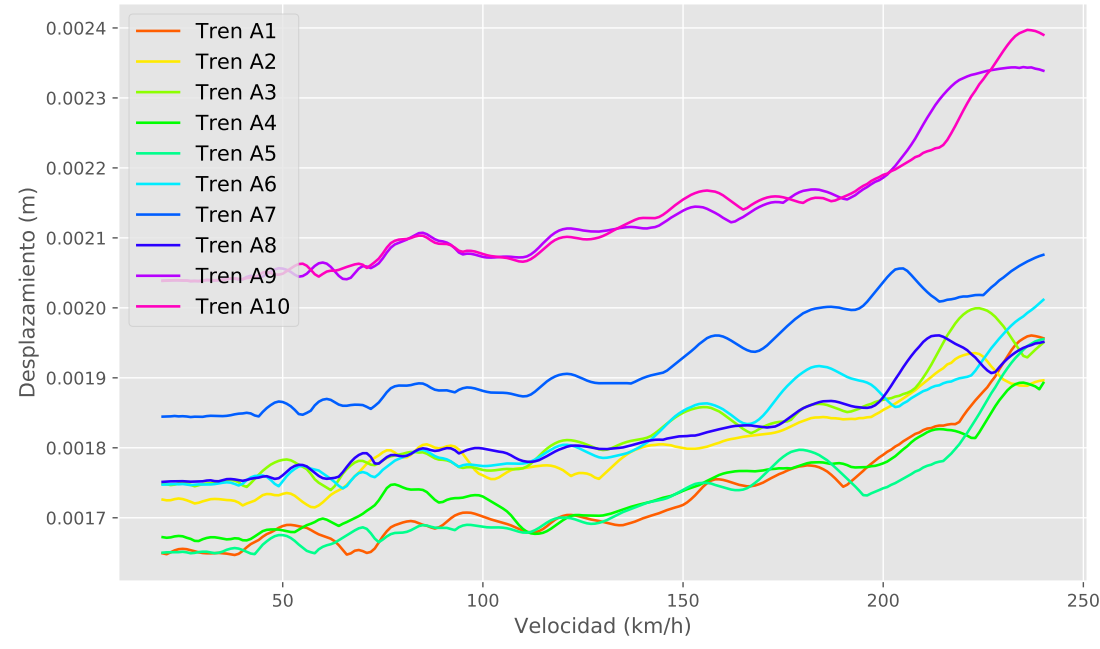

A.7. Resultados para $E_{p} / G_{s}=10000$

\section{A.7.1. Resultados de aceleración}

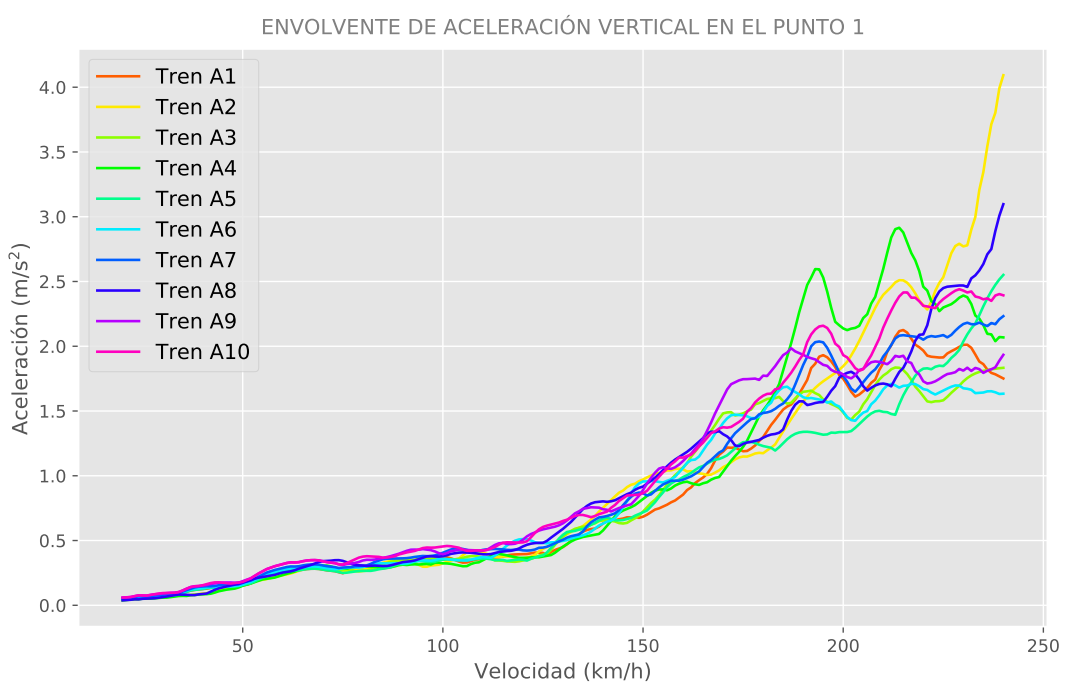


ENVOLVENTE DE ACELERACIÓN VERTICAL EN EL PUNTO 2

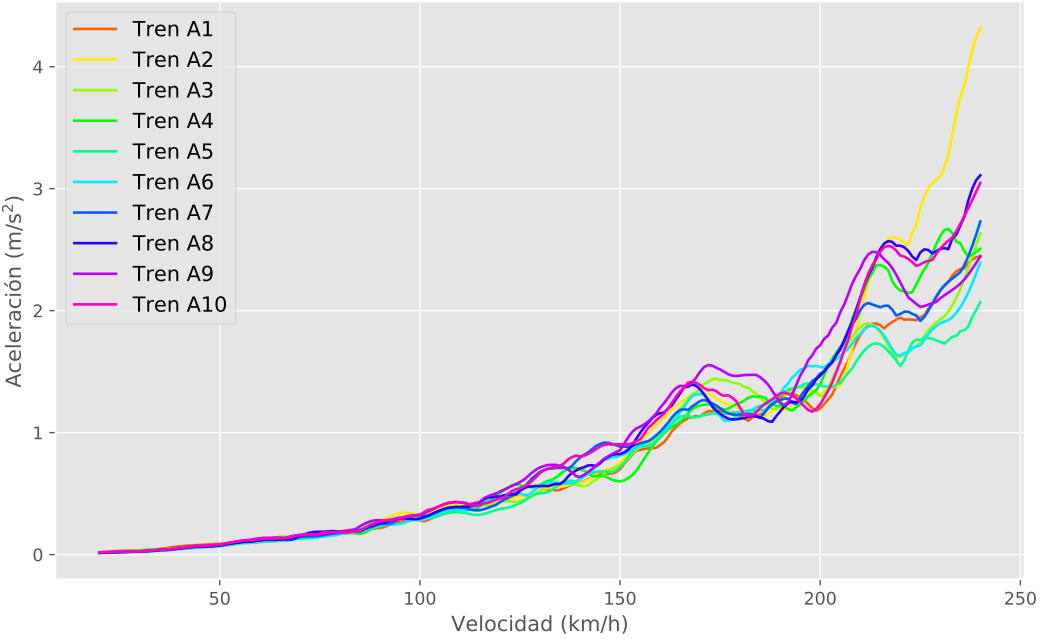

ENVOLVENTE DE ACELERACIÓN VERTICAL EN EL PUNTO 3

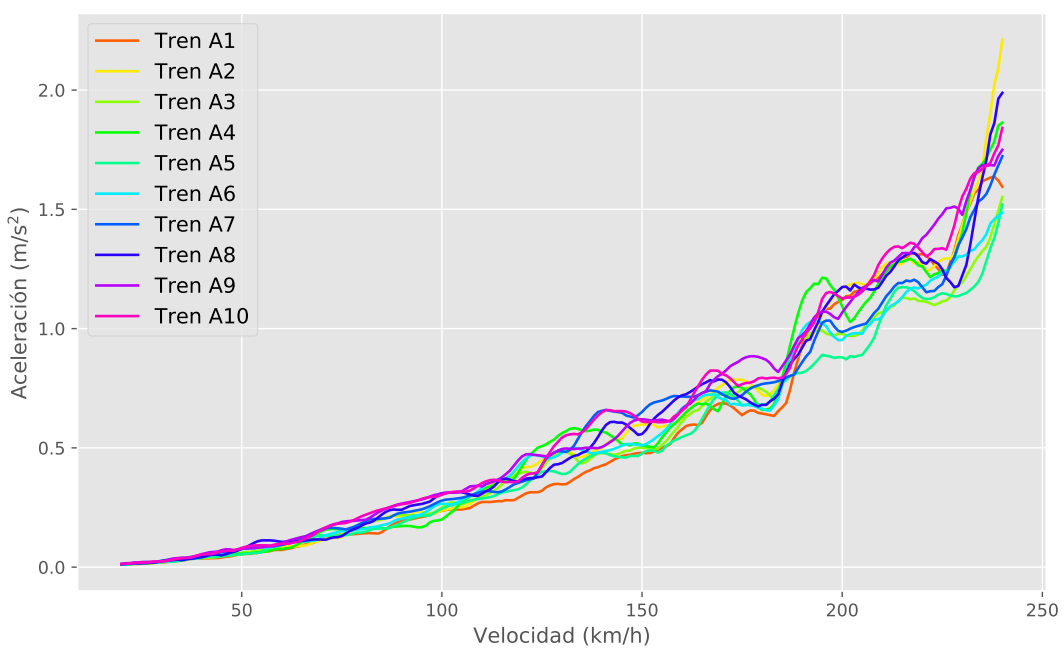


ENVOLVENTE DE ACELERACIÓN VERTICAL EN EL PUNTO 4

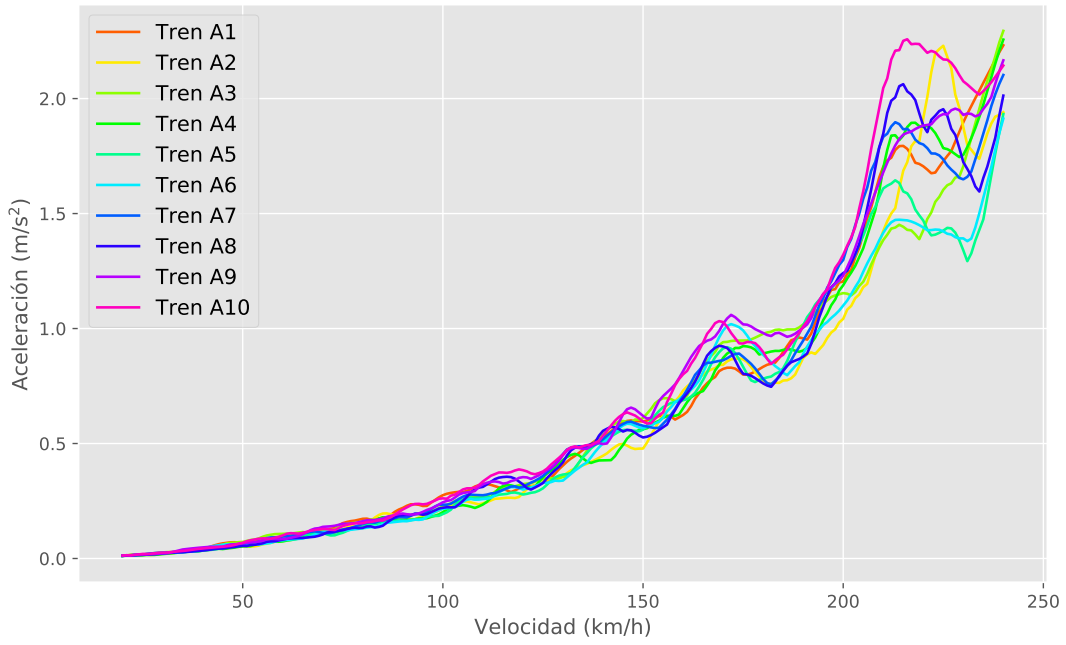

ENVOLVENTE DE ACELERACIÓN VERTICAL EN EL PUNTO 5

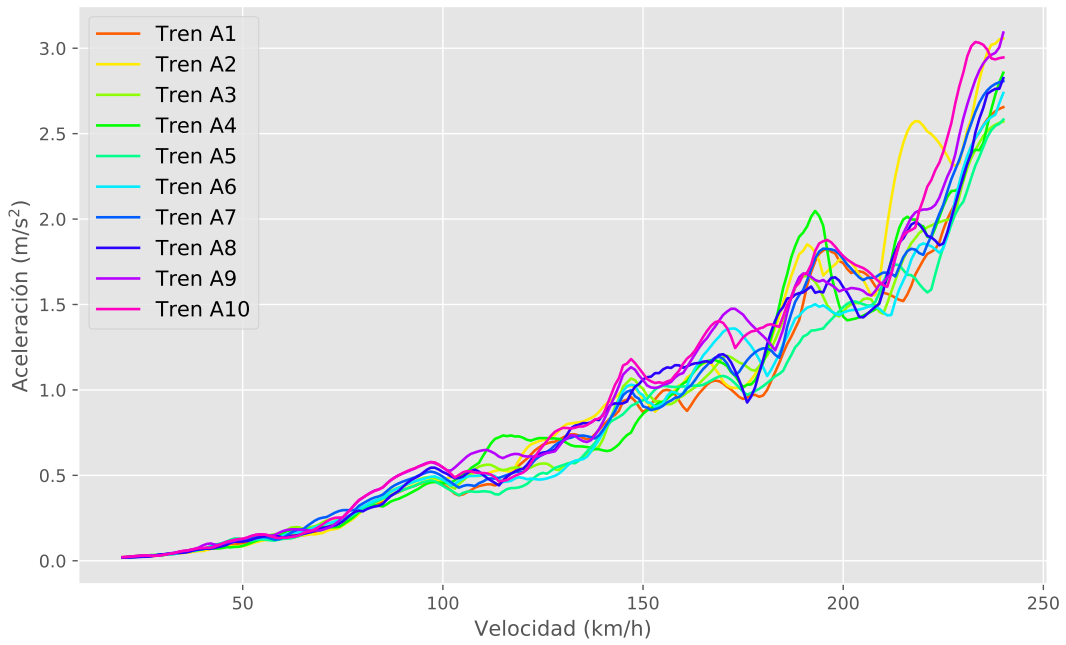


ENVOLVENTE DE ACELERACIÓN VERTICAL EN EL PUNTO 6

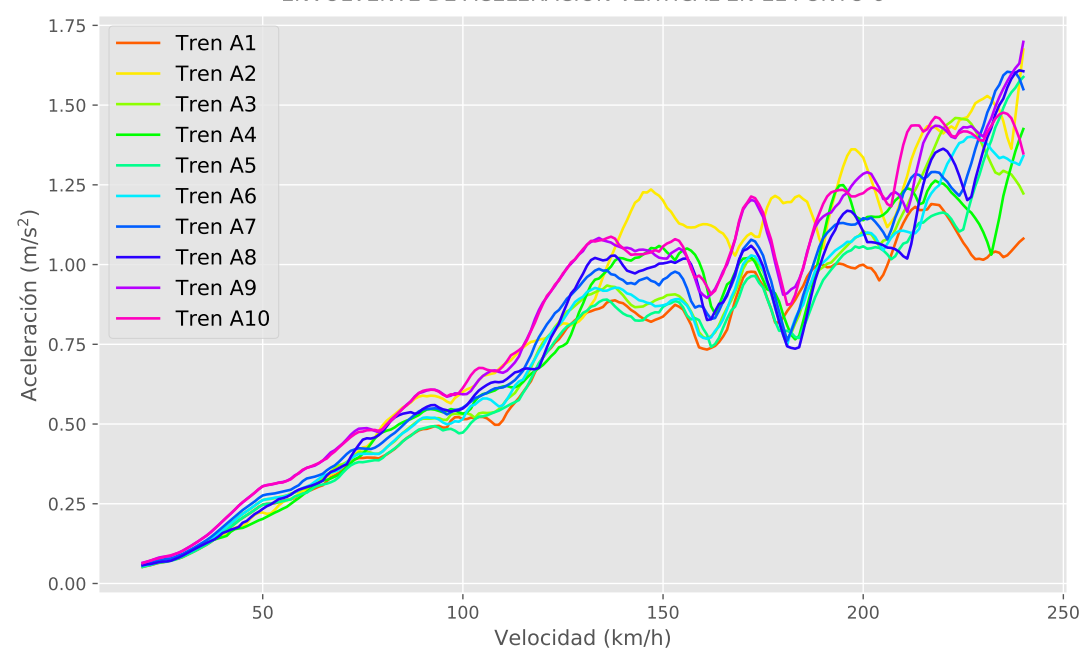

ENVOLVENTE DE ACELERACIÓN VERTICAL EN EL PUNTO 7

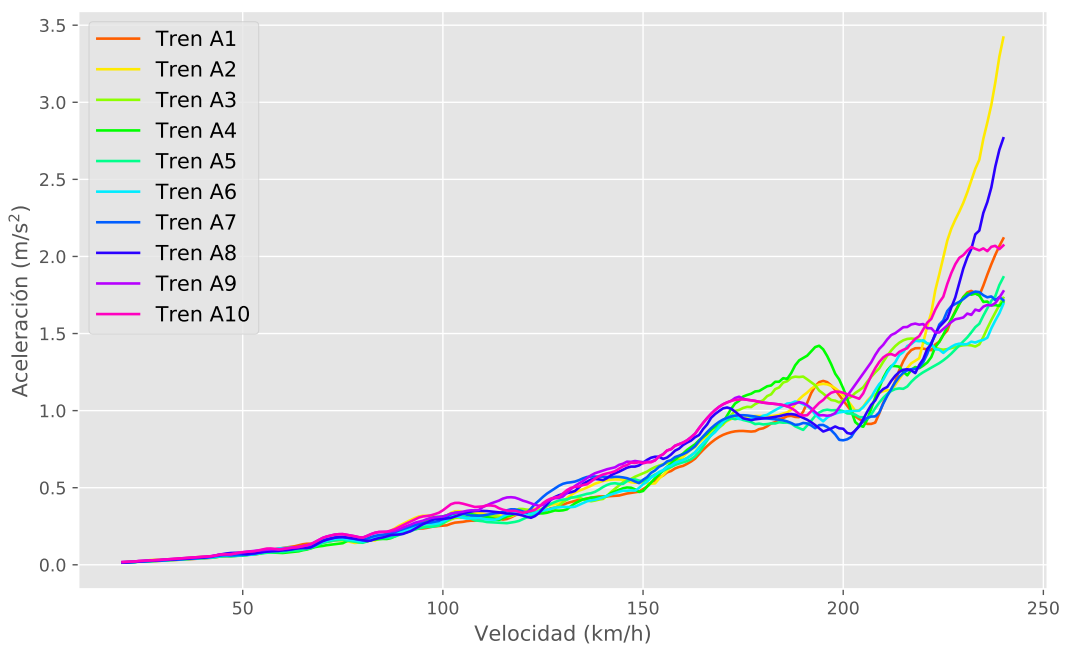


ENVOLVENTE DE ACELERACIÓN VERTICAL EN EL PUNTO 8

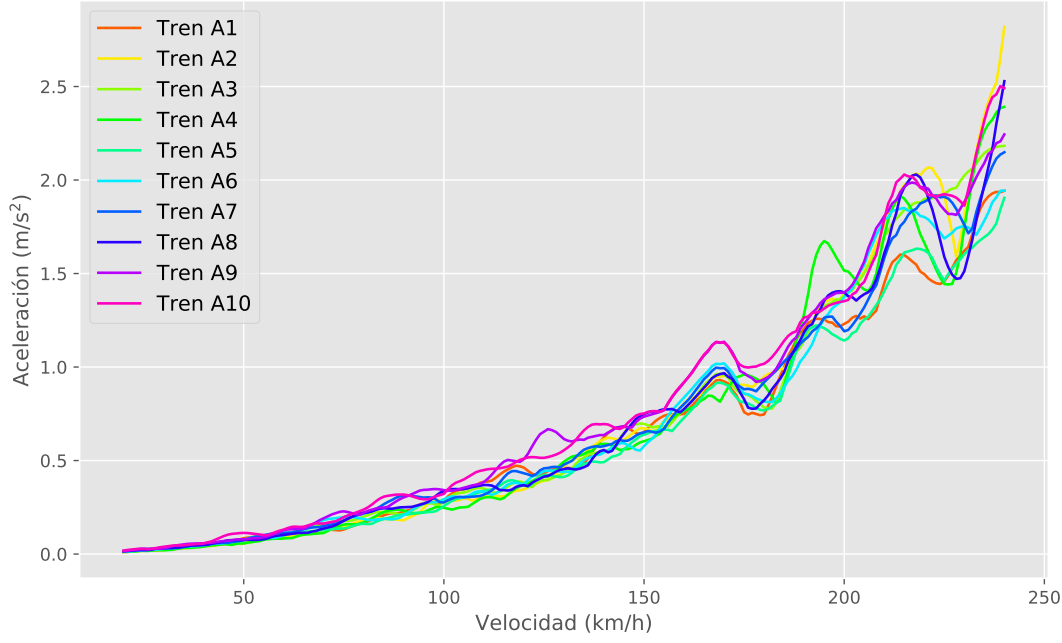

ENVOLVENTE DE ACELERACIÓN VERTICAL EN EL PUNTO 9

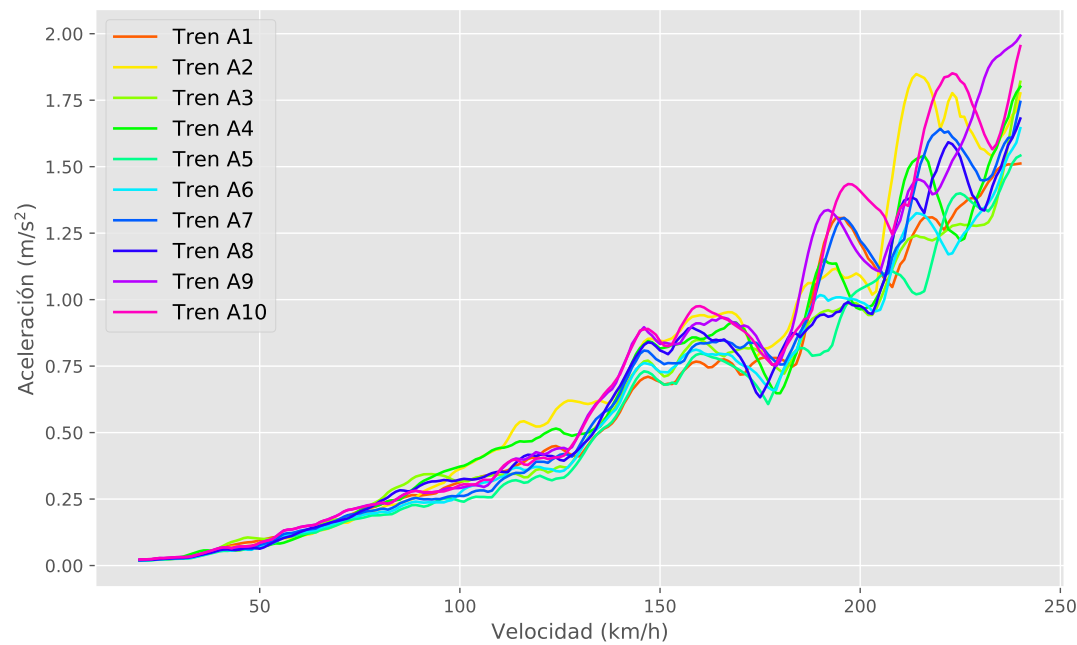


A.7.2. Resultados de desplazamiento
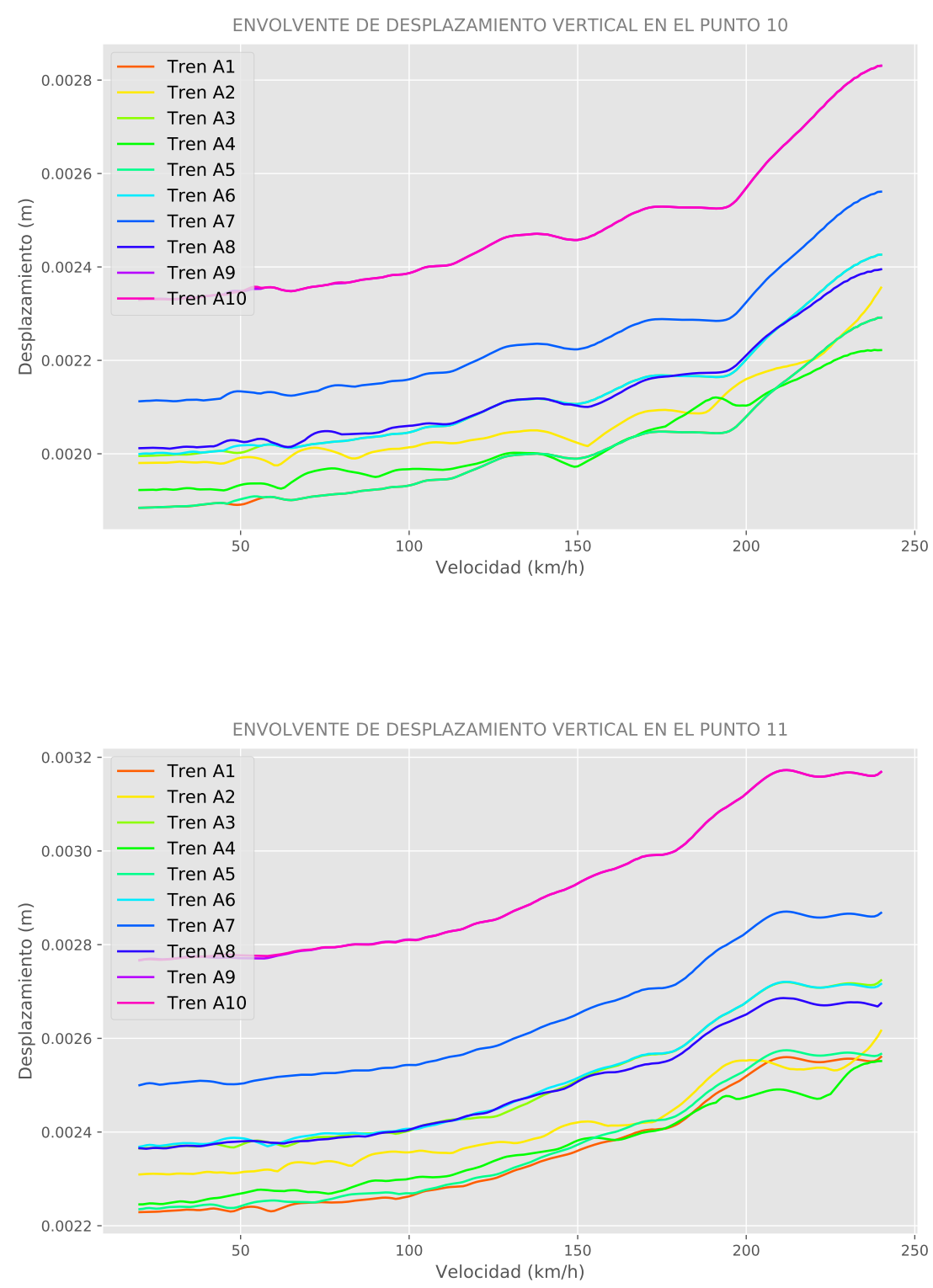
ENVOLVENTE DE DESPLAZAMIENTO VERTICAL EN EL PUNTO 12

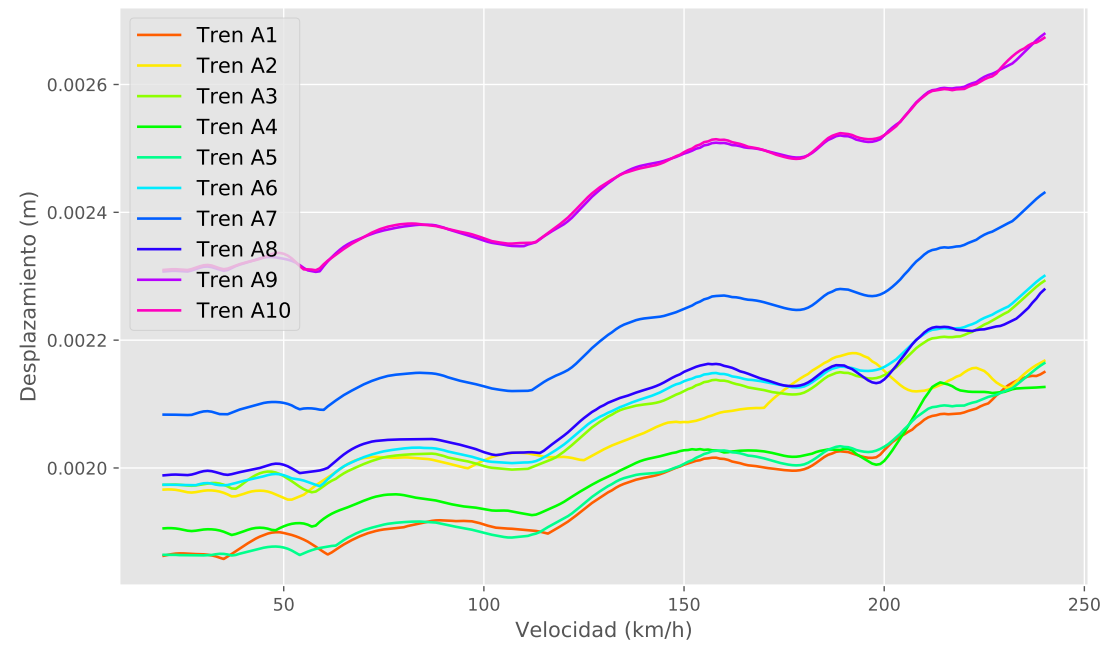





\section{Bibliografía}

[1] UNE-EN 1991-2: 2003. Eurocódigo 1: Acciones en estructuras. Parte 2: Cargas de tráfico en puentes, septiembre 2003.

[2] Instrucción de Acciones a considerar en el proyecto de Puentes de Ferrocarril (IAPF), 2011.

[3] G. W. Blaney, E.Kausel, and J. M. Roesset. Dynamic stiffness of piles. 2nd Int. Conf. Numerical Methods in Geomech, pages 1001-1012, junio 1976. Nueva York.

[4] A. Doménech, M.D. Martínez-Rodrigo, A. Romero, and P. Galvín. On the basic phenomenon of soil-structure interaction on the free vibration response of beams: Application to railway bridges. Engineering Structures, 125:254-265, julio 2016.

[5] Enrique García Macías. Dinámica de puentes arco atirantados para el ferrocarril de alta velocidad. estudio de un caso, planteamiento de alternativas y conclusiones para el diseño. Proyecto Fin de Carrera. Ingeniería de Caminos Canales y Puertos. Universidad de Granada, septiembre 2012.

[6] A.M. Kaynia and E. Kausel. Dynamic stiffness and seismic response of sleeved piles. Report No. R80-12, page 54, mayo 1980.

[7] A.M. Kaynia and E. Kausel. Dynamic behavior of pile groups. 2nd Int. Conf. on Numerical Methods in Offshore Piling, 1982. Austin, Texas.

[8] T. Kobori, R. Minai, and K.Baba. Dynamic behaviour of a laterally loaded pile. 9th Int. Conf. Soil Mech., 1977. Tokio, Japón.

[9] R. L. Kuhlemeyer. Static and dynamic laterally loaded piles. Journal of the Geotechnical Engineering Division, 105(GT2):289-304, 1979.

[10] Guillermo M. Álamo, Juan J. Aznárez, Luis A. Padrón, Alejandro E. Martínez Castro, Rafael Gallego, and Orlando Maeso. Efficient numerical model for the computation of impedance functions of inclined pile groups in layered soils. Engineering Structures, 126:379-390, noviembre 2016.

[11] Guillermo M. Álamo, Juan J. Aznárez, Luis A. Padrón, Alejandro E. Martínez Castro, and Orlando Maeso. Importance of using accurate soil profiles for the estimation of pile kinematic input factors. Journal of Geotechnical and Geoenvironmental Engineering, 145(8), junio 2019.

[12] Mahir Ülker Kaustell, Raid Karoumi, and Costin Pacoste. Simplified analysis of the dynamic soil-structure interaction of a portal frame railway bridge. Engineering Structures, 32:3692-3698, septiembre 2010. 
[13] Patricia Maldonado Salguero. Análisis dinámico de pérgolas para el ferrocarril de alta velocidad. Proyecto Fin de Carrera. Ingeniería de Caminos Canales y Puertos. Universidad de Granada, enero 2014.

[14] A.E. Martínez Castro, P. Museros, and A. Castillo Linares. Semi-analytic solution in the time domain for non-uniform multi-span bernoulli-euler beams traversed by moving loads. Journal of Sound and Vibration, 294:278-297, 2006.

[15] M.D. Martínez-Rodrigo, P. Galvín, A. Doménech, and A. Romero. Effect of soil properties on the dynamic response of simply-supported bridges under railway traffic through coupled boundary element-finite element analyses. Engineering Structures, 170: 78-90, 2018.

[16] P. Museros, A.E. Martínez Castro, and A. Castillo Linares. Semi-analytic solution for kirchhoff plates traversed by moving loads. Actas del Eurodyn 2005, Paris, 2005.

[17] T. Nogami. Dynamic stiffness and damping of pile groups in inhomogeneous soil. Proc. of Session on Dynamic Response of Pile Foundations: Ana- lytical Aspects, pages 31-52, octubre 1980.

[18] T. Nogami and M. Novak. Soil-pile interaction in vertical vibration. Journal of Earthquake Engineering and Structural Dynamics, 4:277-293, enero - marzo 1976.

[19] M. Novak. Dynamic stiffness and damping of piles. Canadian Geotechnical Journal, II: 574-598, 1974.

[20] M. Novak. Vertical vibration of floating piles. Journal of the Engineering Mechanics Division, 103(EM1):153-168, febrero 1977.

[21] M. Novak and F. Aboul-Ella. Impedance functions of piles in layered media. Journal of the Engineering Mechanics Division, 104:643-661, junio 1978.

[22] M. Novak and F. Aboul-Ella. Stiffness and damping of piles in layered media. Proc. Earthq. Engrg. and Soil Dyn, pages 704-719, 1978. Pasadena, California.

[23] M. Novak and R. F. Grigg. Dynamic experiments with small pile foun- dations. Canadian Geotechnical Journal, 13(4):372 - 385, 1976.

[24] M. Novak and J.F. Howell. Torsional vibration of pile foundations. Journal of the Geotechnical Engineering Division, 103(GT4):271-285, abril 1977.

[25] M. Novak and J.F. Howell. Dynamic response of pile foundations in torsion. Journal of the Geotechnical Engineering Division, 104(GT5):535-552, 1978.

[26] M. Novak and M. Sheta. Approximate approach to contact problems of piles. Proc. Geotechnical Engineering Division, pages 53-79, octubre 1980.

[27] M. Novak, T. Nogami, and F. Aboul-Ella. Dynamic soil reactions for plane strain case. Journal of the Engineering Mechanics Division, 104(EM4):953-959, 1978.

[28] Milos Novak, M. ASCE, and Bahaa El Sharnouby. Stiffness constants of single piles. J. Geotech. Engrg, 109(7):961-974, 1983.

[29] H.G. Poulos. Behavior of laterally loaded piles. ii pile groups. Journal of the Soil Mechanics Foundations Division, 97(SM5):733-751, 1971. 
[30] H.G. Poulos and E.H. Davis. Pile foundations analysis and design. John Wiley and Sons, page 397, 1980.

[31] M.F. Randolph and H.G. Poulos. Estimating the flexibility of offshore pile groups. Proc. of the Conf. on Numerical Methods in Offshore Piling, page 16, 1982. Universidad de Texas, Austin.

[32] J.M. Roesset. Stiffness and damping coefficients of foundations. ASCE National Convention, pages 1-30, octubre 1980.

[33] A. Romero, M. Solís, J. Domínguez, and P. Galvín. Soil-structure interaction in resonant railway bridges. Soil Dynamics and Earthquake Engineering, 47:108-116, julio 2012.

[34] M. Sheta and M. Novak. Vertical vibration of pile groups. Journal of the Geotechnical Engineering Division, 108(GT4):570-590, abril 1982.

[35] H. Tajimi. Dynamic analysis of a structure embedded in an elastic stratum. Proc. 4th World Conference Earthquake Engineering, 1969. Chile.

[36] V.M. Trbojevic, J. Marli, R. Danish, and K. Delink. Pile-soil-pile interaction analysis for pile groups. 6th SMIRT, 1981. París, Francia.

[37] Jokin Ugarte González. Análisis del comportamiento dinámico de tableros de puentes pérgola de ferrocarriles en líneas de alta velocidad. Trabajo Fin de Máster. Ingeniería de Caminos Canales y Puertos. Universidad Politécnica de Madrid, 2013.

[38] G. Waas and H.G. Hartmann. Pile foundations subjected to dynamic horizontal loads. Modelling and Simulation of Large Scale Structural Systems, page 17, septiembre 1981. Capri, Italia.

[39] J.P. Wolf and G.A. von Arx. Impedance functions of a group of vertical piles. Proceedings of ASCE Specialty Conf. on Earthquake Engrg. and Soil Dynamics, pages 1024-1041, 1978. Pasadena, California.

[40] Abbas Zangeneh, Jean-Marc Battini, Costin Pacoste, and Raid Karoumi. Fundamental modal properties of simply supported railway bridges considering soil-structure interaction effects. Soil Dynamics and Earthquake Engineering, 121:212-218, 2019. 